\title{
Effects of Internal Waves on Low Frequency, Long Range, Acoustic Propagation in the Deep Ocean
}

\author{
by \\ Jinshan $\mathrm{Xu}$ \\ B.S., Electrical engineering, Ocean University of Qingdao, China (1994) \\ S.M., Physical Oceanography, Ocean University of Qingdao, China (1999) \\ Submitted in partial fulfillment of the requirements for the degree of \\ Doctor of Philosophy \\ at the \\ MASSACHUSETTS INSTITUTE OF TECHNOLOGY \\ and the \\ WOODS HOLE OCEANOGRAPHIC INSTITUTION \\ September 2007 \\ (C)2007 Jinshan Xu. All rights reserved.

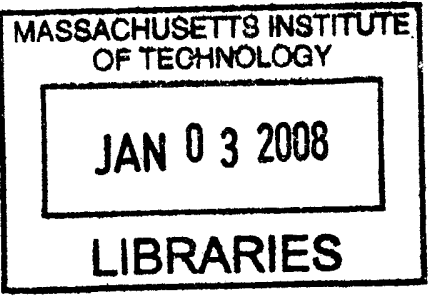 \\ BARKER
}

The author hereby grants to MIT and WHOI permission to reproduce paper and electronic copies of this thesis in whole or in part and to distribute them publicly.

Author

Joint Program in Oceanography/Applied Ocean Science and Engineering Massachusetts Institute of Technology and Woods Hole Oceanographic Institution August 6, 2007

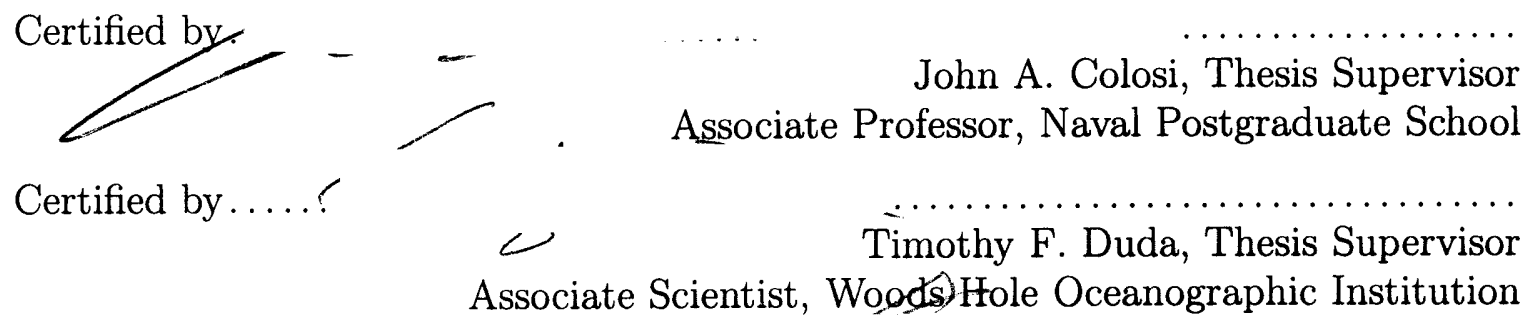

Accepted by

Henrik Schmidt

Chairman, Jolkt Committee for Applied Ocean Science and Engineering Massachusetts Institute of Technology/Woods Hole Oceanographic Institution 
$+\ldots$ 


\title{
Effects of Internal Waves on Low Frequency, Long Range, Acoustic Propagation in the Deep Ocean
}

by

Jinshan Xu

\begin{abstract}
Submitted to the MIT Department of Mechanical Engineering and to the WHOI Department of Applied Ocean Science and Engineering on August 6, 2007, in partial fulfillment of the requirements for the degree of Doctor of Philosophy
\end{abstract}

\begin{abstract}
This thesis covers a comprehensive analysis of long-range, deep-ocean, low-frequency, sound propagation experimental results obtained from the North Pacific Ocean. The statistics of acoustic fields after propagation through internal-wave-induced sound-speed fluctuations are explored experimentally and theoretically.

The thesis starts with the investigation of the North Pacific Acoustic Laboratory 98-99 data by exploring the space-time scales of ocean sound speed variability and the contributions from different frequency bands. The validity of the Garret \& Munk internal-wave model is checked in the upper ocean of the eastern North Pacific. All these results impose hard bounds on the strength and characteristic scales of sound speed fluctuations one might expect in this region of the North Pacific for both internal-wave band fluctuations and mesoscale band fluctuations.

The thesis then presents a detailed analysis of the low frequency, broadband sound arrivals obtained in the North Pacific Ocean. The observed acoustic variability is compared with acoustic predictions based on the weak fluctuation theory of Rytov, and direct parabolic equation Monte Carlo simulations. The comparisons show that a resonance condition exists between the local acoustic ray and the internal wave field such that only the internal-waves whose crests are parallel to the local ray path will contribute to acoustic scattering: This effect leads to an important filtering of the acoustic spectra relative to the internal-wave spectra. We believe that this is the first observational evidence for the acoustic ray and internal wave resonance.

Finally, the thesis examined the evolution with distance, of the acoustic arrival pattern of the off-axis sound source transmissions in the Long-range Ocean Acoustic Propagation EXperiment. The observations of mean intensity time-fronts are compared to the deterministic ray, parabolic equation (with/without internal waves) and (one-way coupled) normal mode calculations. It is found the diffraction effect is dominant in the shorter-range transmission. In the longer range, the (internal wave) scattering effect smears the energy in both the spatial and temporal scales and thus has a dominant role in the finale region.
\end{abstract}

Thesis Supervisor: John A. Colosi

Title: Associate Professor, Naval Postgraduate School

Thesis Supervisor: Timothy F. Duda

Title: Associate Scientist, Woods Hole Oceanographic Institution 


\section{Acknowledgments}

During one of early evenings months ago, while I was in my living room looking out from the windows at the Charles River and the city under the golden sunlight, I wondered why I had never noticed how gorgeous the view was before. I was saddened by the thought of leaving MIT/Boston. I realized what a tremendous opportunity and experience I've had here in my six years in the MIT/WHOI joint program. Over these years, I have been fortunate to get to know and work with a lot of people from whom I've learned a great deal.

First I would like to thank my advisors, Prof. John Colosi and Dr. Tim Duda for sharing their ideas and insights, challenging and providing encouragement to me throughout the entire project. I want to express my heartfelt gratitude to them for their guidance and advice during both the research and writing of this thesis. I am also very grateful to my thesis committee members, Profs. James Mercer, Henrik Schmit, and Arthur Baggeroer. Thank you, Dr. Andone Lavery, for being my defense chair, and for reading through my thesis in such a short period.

This research was made possible by the experimental work carried out by the ATOC Group. I am fortunate to have participated in three research cruises from 2004 to 2005 in the North Pacific Ocean and have participated in obtaining real data from the ocean. During my last two years research, I have received kind help from Drs. Rex Andrew, Mike Wolfson in APL of Univerisity Of Washington, as well as Drs. Peter Worcester, Matt Dzieciuch, and Lora Van Uffelen in Scripps of UCSD, and Andrey Morozov, Eddie Shceer in WHOI.

I am grateful to my many friends for their encouragement and support. The list could be really long, but I specially want to thank Wenyu Luo, Joseph Sikora, Steven $\mathrm{Xu}$, Chen Xiao, Tony Lin, Tatsuya Arai, Stephen Conrad, Winnie Ye, Li Yang, Bei Zeng and Jingen Xiang, who have made my very last year of graduate study still enjoyable.

The funding that made this research possible came from the Office of Naval Research, and the WHOI Academic Programs Office. In addition to funding, Julia Westwater, Marsha Gomes and the APO couldn't do it better. A big thank-you for their kind help and encouragement throughout the years, as well as Ronni Schwartz on the 9th floor of the Green Building in MIT.

Finally, I would like to thank my parents and my brother and sisters: Jiansheng, Ping, and Shannon for their love and support. You are truly a blessing in my life, none of this 
would have been possible without you.

Thank you all...

Jinshan 


\section{Contents}

$\begin{array}{llr}1 & \text { Introduction } & \mathbf{2 1}\end{array}$

1.1 Background . . . . . . . . . . . . . . . . . . . 21

1.2 Context and Motivation for this Study . . . . . . . . . . . . 22

1.2.1 Acoustic Fluctuations . . . . . . . . . . . . . . . . 22

1.2 .2 Ocean Modeling . . . . . . . . . . . . . . . . 26

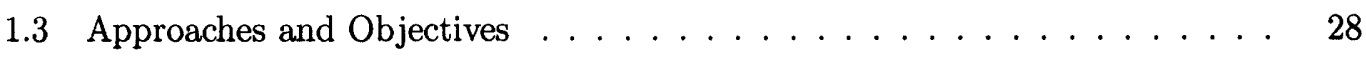

2 Observations of Deep Water, Upper Ocean Sound Speed Structure in the Eastern North Pacific Ocean $\quad 31$

2.1 Introduction . . . . . . . . . . . . . . . . . . . 31

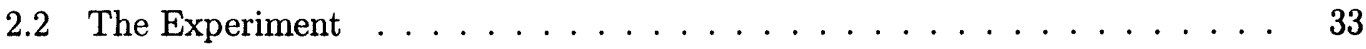

2.2 .1 Moored Observations . . . . . . . . . . . . . . . . . . 33

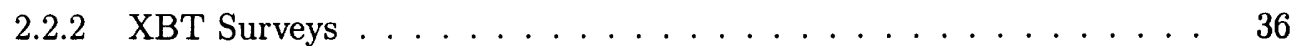

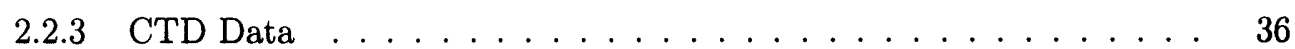

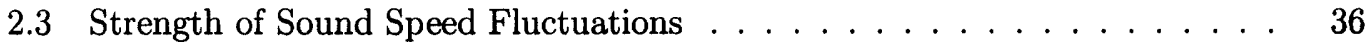

2.3.1 Internal Wave and Mesoscale Frequency Bands . . . . . . . . . . 41

2.3.2 Seasonal Variation in the Internal Waves Band . . . . . . . . . 41

2.3.3 Internal Wave Band Model Comparisons . . . . . . . . . . . . . . . . 46

2.3 .4 Internal Tide Variability . . . . . . . . . . . . . . . . . 46

2.3.5 Vertical structure of sound speed variance derived from XBT data . 49

2.4 Spectral Analysis of Sound Speed and Temperature . . . . . . . . . . . 52

2.4.1 Frequency Spectra of Sound Speed . . . . . . . . . . . . . . 52

2.4.2 Vertical Wavenumber Spectra of Potential Temperature . . . . . 56

2.4.3 Horizontal and Vertical Wavenumber Spectra of Temperature . . . . 60 


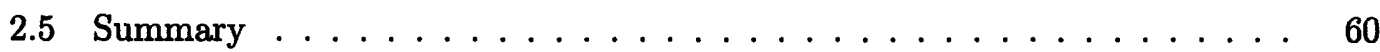

3 Observations and Modeling of Low-frequency Sound Wave Propagation in the Random Deep Ocean 65

3.1 Introduction . . . . . . . . . . . . . . . . 65

3.2 AET Experiment and Reduction of the data to Phase and Amplitude . . . 67

3.2 .1 Ray Identification . . . . . . . . . . . . . . . . 68

3.2.2 Arrival Selection and Processing . . . . . . . . . . . . 72

3.3 Observed Phase and Intensity Fluctuations . . . . . . . . . . . 76

3.3.1 Moments ..................... 76

3.3.2 Frequency Spectra . . . . . . . . . . . . . . . 80

3.3.3 Vertical Wavenumber Spectra . . . . . . . . . . . . . . . 82

3.4 Modeling . . . . . . . . . . . . . . . . . . . 84

3.4.1 MZ Theory and Predictions . . . . . . . . . . . . . . 84

3.4.2 Narrowband Model . . . . . . . . . . . . . . . . . . . . . . 89

3.4 .3 Broadband Model $\ldots \ldots \ldots \ldots 99$

3.5 Data-Model Comparisons . . . . . . . . . . . . . . . . . . . 101

3.5 .1 Moments . . . . . . . . . . . . . . . . . . 101

3.5 .2 Frequency Spectra . . . . . . . . . . . . . . . . . 102

3.5.3 Vertical Wavenumber Spectra . . . . . . . . . . . . . . . 103

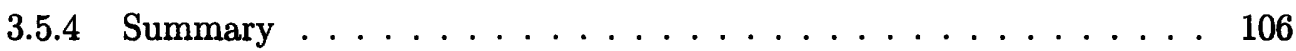

3.6 Summary . . . . . . . . . . . . . . . . . . . . . . 108

4 The Range Evolution of the Mean Intensity of the LOAPEX Off-axis $\begin{array}{ll}\text { Source Transmissions } & 109\end{array}$

4.1 Introduction . . . . . . . . . . . . . . . . . . . . 109

4.1.1 Science Objectives of LOAPEX experiment . . . . . . . . . 110

$4.1 .2 \quad$ Approach . . . . . . . . . . . . . . . . 111

4.2 LOAPEX Experiment Data Collection . . . . . . . . . . . . . . 116

4.2.1 Acoustic Transmission . . . . . . . . . . . . . . . 116

4.2.2 Environmental Measurements . . . . . . . . . . . . . . . . . 119

4.3 LOAPEX Data Processing . . . . . . . . . . . . . . . . . . . . . . . 124

4.3.1 Doppler . . . . . . . . . . . . . . . . . . . . . . 124 
4.3.2 Estimation of the Transmission Loss at Receiver Distance . . . . . . 124

4.3.3 Estimation of Acoustic Wave Intensity Recorded from VLA . . . . 126

4.3 .4 Mean Wave Front Intensity . . . . . . . . . . . . . . 127

4.4 Numerical Simulations . . . . . . . . . . . . . . . . . . . . . . 127

4.4.1 Ray method- Eigenray Code . . . . . . . . . . . . . . 130

4.4.2 Normal Modes Method - C-SNAP . . . . . . . . . . . . . . . 130

4.4.3 Parabolic Equation method - RAM . . . . . . . . . . . . . . . 131

4.4.4 Comparison between the different numerical simulations . . . . . . 131

4.5 Comparison between Observations and Numerical Simulations . . . . . . . 132

4.5.1 Mean Wave Front Intensity in the Finale Region . . . . . . . . 132

4.5.2 LOAPEX Time Extension into the Finale Region . . . . . . . . . . 139

4.6 Summary . . . . . . . . . . . . . . . . . . . 152

5 Conclusions and Future Directions 153

5.1 Thesis Contributions . . . . . . . . . . . . . . . . . 153

5.2 Future Work . . . . . . . . . . . . . . . . . 155

5.2 .1 Data Processing . . . . . . . . . . . . . 155

5.2.2 Numerical Modeling: Ocean Modeling and Acoustic Propagation Modeling ........................... 155

5.2 .3 Theory Prediction . . . . . . . . . . . . . . . 157

$\begin{array}{ll}\text { A Maximum-Likelihood Estimate } & 161\end{array}$

$\begin{array}{ll}\text { B Rytov Method } & 163\end{array}$

B.1 Rytov General Solution to Stochastic Wave Equation . . . . . . . . . 163

B.2 Rytov Theory for Incident Plane Wave and Small Angle Propagation . . . . 164

B.3 Spectra of Phase and log-amplitude: No waveguide . . . . . . . . . 166

B.4 Spectra of Phase and log-amplitude: Waveguide . . . . . . . . . 167

$\begin{array}{ll}\text { C Computation of the Fresnel Zone } & 171\end{array}$

$\begin{array}{ll}\text { D Parabolic Equation Method } & 173\end{array}$

D.1 Derivation of Standard Parabolic Equation . . . . . . . . . . 173

D.2 The Split-Step Fourier Algorithm . . . . . . . . . . . . . . . 174 
E Vertical Line Array (VLA) Calibration $\quad 177$

E.1 System Sensitivity . . . . . . . . . . . . . . . . . . . . 177

E.2 Signal Processing Gain . . . . . . . . . . . . . . . . . . . 178

F Ray Dynamics : Travel Time Statistics and Time Spreading Scale $\quad 179$

F.1 Travel Time Statistics . . . . . . . . . . . . . . . . . . . . . 179

F.1.1 Upper Turning Point Model $\ldots \ldots \ldots$. . . . . . . . . 180 


\section{List of Figures}

2-1 NPAL 98 - 99 acoustic transmission path between the Kausi Source and Sur Ridge receiver with North Pacific ocean bathymetry map superimposed. . . 34

2-2 The diagram of eastern mooring configuration of sensors. . . . . . . . . 35

2-3 A 10-day timeseries of temperature and salinity records from eastern mooring. $\quad 37$

2-4 A 750-m depth XBT survey for the purpose of mesoscale mapping during the IW98 and IW99 cruises. . . . . . . . . . . . . . . . . . . . . 38

2-5 The first three "high resolution" XBT surveys during the IW98 cruise. . . 39

2-6 The buoyancy frequency profiles as a function of range between the source and receiver, derived from the CTD casts along the transect during the IW99 cruise.

2-7 An example of frequency spectrum of the sound speed in the East Mooring at the depth $490 \mathrm{~m} . \ldots \ldots \ldots \ldots \ldots \ldots \ldots$

2-8 An example of separating the internal wave and mesoscale frequency bands from the original time series. $\ldots \ldots \ldots \ldots \ldots$

2-9 The internal wave and mesoscale sounds speed variance as a function of depth over the whole mooring deployment. . . . . . . . . . . . . 44

2-10 The seasonal variation of the sound speed variance as function of depth in the super-inertial band. . . . . . . . . . . . . . . . . . 45

2-11 A comparison between the sound speed variance and canonical model. . . . 47

2-12 Sound speed variance due to the internal tide is subtracted from the total sound speed variance. M2 tide random and deterministic component are separated based on the extension of the power law continuum from high frequency. . . . . . . . . . . . . . . . . . . 
2-13 The spatial sound speed variance as function of depth based on the 5 high resolution XBT surveys in IW98 and IW99 cruise. . . . . . . . . . . 50

2-14 The spatial sound speed variance as function of depth based on the mesoscale XBT surveys in IW98 and IW99 cruise. . . . . . . . . . . . . . . . 51

2-15 Frequency spectrum of the sound speed fluctuation in the sub-inertial band at the different depths for west and east mooring. . . . . . . . . . . . 53

2-16 Comparison between GM model and the frequency spectrum of the sound speed fluctuation in super-inertial band at different depths. . . . . . . . . 55

2-17 The seasonal variation of sound speed spectrum at depths of $160 \mathrm{~m}, 400 \mathrm{~m}$, $640 \mathrm{~m}$ of western and eastern moorings. . . . . . . . . . . . . . 57

2-18 Seasonal variation of the vertical wavenumber spectrum of potential temperature for western and eastern moorings. . . . . . . . . . . . . . 58

2-19 Vertical wavenumber spectrum of potential temperature in different frequency bands for west and east moorings. . . . . . . . . . . . . . . . 59

2-20 Vertical wavenumber spectrum of temperature from five high resolution XBT surveys of IW98 and IW99 cruises. . . . . . . . . . . . . . . . . 61

2-21 Vertical wavenumber spectrum of temperature gradient from five high resolution XBT surveys of IW98 and IW99 cruises. . . . . . . . . . . . 62

2-22 Horizontal wavenumber spectrum of temperature from five high resolution XBT surveys for IW98 and IW99 cruise. . . . . . . . . . . . .

3-1 Map showing the location of the AET experiment in the North Pacific. Also shown are sound speed and buoyancy frequency profiles derived from CTD casts at the deployment and recovery of NVLA. . . . . . . . . . . . . 69

3-2 Two samples of sound pressure field recorded in the Near VLA. . . . . . 70

3-3 Range independent ray simulation result based on AET sound speed profile. 71

3-4 This plot is showing the eigen ray trace and time front for the $87 \mathrm{~km}$ range transmission and near VLA receiver configuration. . . . . . . . 71

3-5 The estimated pulse shape for two arrivals. . . . . . . . . . . . . 73

3-6 One example of resolving two arrivals by using maximum likelihood estimation method. . . . . . . . . . . . . . . . . . . 74

3-7 The RMS of intensity as function of depth for two arrivals. . . . . . . . . 75 
3-8 This figure displays the intensity and phase fluctuation as function of time ( $\mathrm{x}$ axis) and depth (y axis) of two wave fronts in 20-minute transmission. . $\quad 77$

3-9 Figure shows the time series of intensity of hydrophone 1, 10 and 20 during 6 days observation period with the depths of around $900 \mathrm{~m}, 1250 \mathrm{~m}$ and $1600 \mathrm{~m}$, respectively. ....................... 81

3-10 Frequency spectra of log-amplitude and phase of Index -3 and Index 4 arrivals. 83

3-11 Vertical wavenumber spectra of phase and log-amplitude of Index -3 and Index 4 arrivals. . . . . . . . . . . . . . . . . . . . . 84

3-12 Raypaths (upper), Fresnel zone $R_{f z}(x)$ (middle), and low frequency cutoff $\omega_{L}(x)$ (lower) for two eigenrays with ID's -3 (left) and +4 (right). . . . . 88

3-13 Rytov prediction of frequency spectrum of wave fronts' phase and log-amplitude for a $75 \mathrm{~Hz} \mathrm{cw}$ signal propagation through a $87 \mathrm{Km}$ range. . . . . . . . . . 89

3-14 Rytov prediction of vertical wavenumber spectrum of wave fronts' phase and log-amplitude for a $75 \mathrm{~Hz} \mathrm{cw}$ signal propagation through a $87 \mathrm{Km}$ range. $\quad . \quad 90$

3-15 Estimates of the sound-speed profile and the buoyancy profile during the AET experiment. The buoyancy profile was smoothed . . . . . . . . 92

3-16 A realization of the internal-wave displacement for $\zeta_{0}=7.3 \mathrm{~m}$ and $j_{m}=100 . \quad 94$

3-17 A realization of evolved internal-wave displacement field with time step of 120 seconds. . . . . . . . . . . . . . . . . . . . 94

3-18 Internal wave horizontal wave number spectra and vertical wave number spectra as modeled in this study. $\ldots \ldots \ldots \ldots . \ldots \ldots$

3-19 Internal wave frequency spectra as modeled in this study. . . . . . . . . . 95

3-20 Simulation of narrow-band beam propagation. . . . . . . . . . . . 97

3-21 RMS of phase (right panel) and intensity (left panel) as function of depth for ID -3 (blue solid) and ID +4 (red dash) arrivals. . . . . . . . . . . . 97

3-22 Frequency spectra of log amplitude(left panel) and phase (right panel) for ID -3 (red solid)and ID +4 (green dash) arrivals. . . . . . . . . . . 98

3-23 Vertical wavenumber spectra of log amplitude(red solid) and phase (green dash) for ID -3 (left panel) and ID +4 (right panel) arrivals. . . . . . . . 99

3-24 Broad-band simulation with (right panel) and without (left panel) internal wave perturbation: Wave front at $87 \mathrm{~km}$ range. . . . . . . . . . . . 
3-25 RMS of intensity (left panel) and phase (right panel) of two arrivals ID -3 (blue line with dot mark) and ID +4 (red line with star mark) as function of depth. .......................... 100

3-26 Frequency spectra of log amplitude(left panel) and phase (right panel) for ID -3 (blue solid)and ID +4(red dash) arrivals' wavefront fluctuation. . . . . 101

3-27 Vertical wavenumber spectra of log amplitude (red dash) and phase (blue solid) for ID -3 (left panel)and ID +4 (right panel) arrivals' wave front fluctuation. . . . . . . . . . . . . . . . . . . 102

3-28 Comparison of frequency spectra of log-amplitude and phase between observations and Rytov theory prediction. . . . . . . . . . . . . . . . 104

3-29 Frequency spectra of phase and log-amplitude from broadband(red dot), and narrow-band(dash) parabolic equation simulations of sound transmission to 87-km range through random filds of Garrett-Munk internal waves. . . . . . 105

3-30 The vertical wavenumber spectra of log-amplitude and phase. . . . . . . 106

3-31 Vertical wavenumer spectra of log-amplitude (left panels) and phase (right panels) form the MZ theory, and narrowband and broadband Monte Carlo

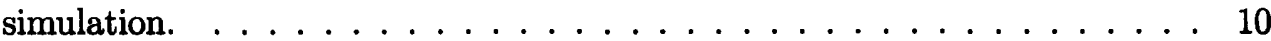

4-1 Experimental geometry. Acoustic paths from the sources $[75-\mathrm{Hz}$ ship-suspended LOAPEX source (red points), moored SPICE04 S1 and S2 250-Hz sources (black) 500 and $1000 \mathrm{~km}$ west of VLA, and Kauai 75- $\mathrm{Hz}$ source] to the receivers (S1 and S2, Navy receivers, the vertical line array, and the BASSEX towed receivers). . . . . . . . . . . . . . . .

4-2 The sound speed profiles at different stations in LOAPEX experiment. The sound speed profiles are derived from the CTD measurement. The red dots on each profiles indicate the sound source depth at each station. The black triangles indicate the sound channel depth at each station. The round-dotmarks on the left indicate the shallow and deep VLA. The sound channel depth is range dependent as being shown here. . . . . . . . . . . 11 
4-3 Modes functions estimated for the LOAPEX experiment. The average sound speed profile is used here. The selected number of modes are displayed in the upper panel. The lower panel shows modes 1 to 6 , with a zoom in display in the lower right panel for the depth range from $300 \mathrm{~m}$ to $400 \mathrm{~m}$. . . . . . 114

4-4 The wavefront at a range of $484 \mathrm{~km}$ broadband transmission simulations. The left two panels display the sound speed profiles with red dots indicating the sound source depth and horizontal line indicating the sound channel depth. The right upper panel shows the simulation with sound source depth of 800 $\mathrm{m}$. The right lower panel shows the simulation with sound source depth of 350 meter. In each simulation plot, there are CSNAP simulation result in color overlapped with eigenray simulations indicated by black dots. . . . 115

4-5 LOAPEX salinity section with potential density contours. . . . . . . . 120

4-6 LOAPEX sound speed section with potential density contours. . . . . . . . 121

4-7 Buoyancy frequency profiles from the LOAPEX CTD section. Curves 1-7 correspond to staions T50, T250, T500,T1000, T1600, T2300, and T3200. Right panel displays upper ocean variability. . . . . . . . . . . . . 122

4-8 Sound speed profiles from the LOAPEX CTD section. Curves 1-7 correspond to staions T50, T250, T500,T1000, T1600, T2300, and T3200. Right panel displays upper ocean variability. $\ldots \ldots \ldots \ldots \ldots$

4-9 Temperature fluctuations from the LOAPEX XBT section. Black ticks in the top of the panel mark where casts were made. Probe depths varied between $760 \mathrm{~m}, 1000 \mathrm{~m}$, and $1830 \mathrm{~m}$. Much of the deep section below $760 \mathrm{~m}$ is filled in by horizontal interpolation. . . . . . . . . . . . .

4-10 The estimated intensity recorded at VLA for LOAPEX transmissions. The spreading loss calculations for a single ray conservatively assume pure spherical spreading in first $10 \mathrm{~km}$ then cylindrical spreading afterward. Attenuation is calculated for the North Pacific Ocean using Lovett $(A=0.055)$. . . . .

4-11 The power spectra density of the sound pressure at different stations. The upper panel indicate the data from upper SVLA, the lower panel indicates the data from lower SVLA. The vertical line in the lower panel indicates the carrier frequency of $68.2 \mathrm{~Hz} \ldots \ldots \ldots \ldots \ldots$ 
4-12 The mean wave front intensity at each station: T250 to T3200 from top to bottom respectively. The colorbars indicate the absolute sound intensity in

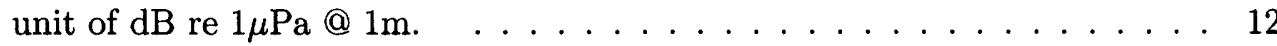

4-13 The simulations of LOAPEX station T250 transmission. From top to bottom, the three panels are PE (RAM) simulations without internal wave, CSNAP simulations without internal wave, and the PE (RAM) simulations with internal wave, respectively. In each simulation, the eigenray simulations are overlapped on with black dot marks.

4-14 The simulations of LOAPEX station T500 transmission. From top to bottom, the three panels are PE (RAM) simulations without internal wave, CSNAP simulations without internal wave, and the PE (RAM) simulations with internal wave, respectively. In each simulation, the eigenray simulations are overlapped on with black dot marks.

4-15 The simulations of LOAPEX station T1000 transmission. From top to bottom, the three panels are PE (RAM) simulations without internal wave, CSNAP simulations without internal wave, and the PE (RAM) simulations with internal wave, respectively. In each simulation, the eigenray simulations are overlapped on with black dot marks. . . . . . . . . . . . . 135

4-16 The simulations of LOAPEX station T1600 transmission. From top to bottom, the three panels are PE (RAM) simulations without internal wave, CSNAP simulations without internal wave, and the PE (RAM) simulations with internal wave, respectively. In each simulation, the eigenray simulations are overlapped on with black dot marks.

4-17 The simulations of LOAPEX station T2300 transmission. From top to bottom, the three panels are PE (RAM) simulations without internal wave, CSNAP simulations without internal wave, and the PE (RAM) simulations with internal wave, respectively. In each simulation, the eigenray simulations are overlapped on with black dot marks. 
4-18 The simulations of LOAPEX station T3200 transmission. From top to bottom, the three panels are PE (RAM) simulations without internal wave, CSNAP simulations without internal wave, and the PE (RAM) simulations with internal wave, respectively. In each simulation, the eigenray simulations are overlapped on with black dot marks. . . . . . . . . . . . . 138

4-19 The comparison of observation data and simulations of LOAPEX station T250. From top to bottom, the three panels are LOAPEX transmission data, CSNAP simulations without internal wave, and the PE (RAM) simulations with internal wave, respectively. In each panel, the eigenray simulations are overlapped on with black dot marks. . . . . . . . . . . . . . . . . . . . 140

4-20 The comparison of observation data and simulations of LOAPEX station T500. From top to bottom, the three panels are LOAPEX transmission data, CSNAP simulations without internal wave, and the PE (RAM) simulations with internal wave, respectively. In each panel, the eigenray simulations are overlapped on with black dot marks. . . . . . . . . . . . . . . 141

4-21 The comparison of observation data and simulations of LOAPEX station T1000. From top to bottom, the three panels are LOAPEX transmission data, CSNAP simulations without internal wave, and the PE (RAM) simulations with internal wave, respectively. In each panel, the eigenray simulations are overlapped on with black dot marks. . . . . . . . . . . . . . . 142

4-22 The comparison of observation data and simulations of LOAPEX station T1600. From top to bottom, the three panels are LOAPEX transmission data, CSNAP simulations without internal wave, and the PE (RAM) simulations with internal wave, respectively. In each panel, the eigenray simulations are overlapped on with black dot marks. . . . . . . . . . . . . . 143

4-23 The comparison of observation data and simulations of LOAPEX station T2300. From top to bottom, the three panels are LOAPEX transmission data, CSNAP simulations without internal wave, and the PE (RAM) simulations with internal wave, respectively. In each panel, the eigenray simulations are overlapped on with black dot marks. . . . . . . . . . . . . 144 
4-24 The comparison of observation data and simulations of LOAPEX station T3200. From top to bottom, the three panels are LOAPEX transmission data, CSNAP simulations without internal wave, and the PE (RAM) simulations with internal wave, respectively. In each panel, the eigenray simulations are overlapped on with black dot marks. . . . . . . . . . . . . 145

4-25 The comparison of observation data and simulations. From top to bottom, the five panels are station T250, T500, T1000, T1600, T2300, and T3200 respectively. In each panel, there are four lines indicating the LOAPEX observation, CSNAP simulation, PE RAM simulation with internal wave, and last ray arrival. . . . . . . . . . . . . . . . . . . . 146

4-26 The time spreading of mean front intensity in the post-finale region at each LOAPEX stations. The upper panel is the LOAPEX observations. The middle panel is the simulations without internal waves. The lower panel is the simulations with internal waves. Note there are only 20-hydrophone data

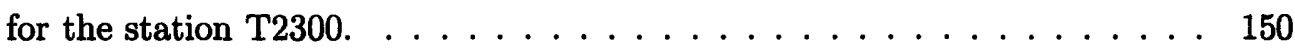

4-27 The time spreading scales for LOAPEX observation and simulations with/without internal waves. Two different scales (linear and $\frac{3}{2}$ ) are indicated for the comparison. . . . . . . . . . . . . . . . . 151 


\section{List of Tables}

3.1 Upper turning points depths and horizontal positions for ID's -3 and +4 . $\quad 72$

3.2 RMS phase, intensity, log-amplitude, and scintillation index of 20 and 40

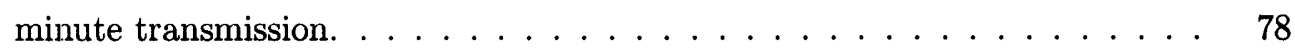

3.3 The source aperture and beam tilt angles(correspond to $2 \theta_{1}$ and $\theta_{2}$ in Eq. 3.33) of narrow-band beams for ID -3 and ID $+4 \ldots \ldots . \ldots 96$

3.4 Comparison Between AET Experiment and Numerical Simulation . . . . 103

4.1 M-Sequence signal parameters . . . . . . . . . . . . . . . 117

4.2 LOAPEX Station coordinates, with range to the deep and shallow VLA and source depths . . . . . . . . . . . . . . . . . . 117

4.3 Signal-to-noise ratios for the $75 \mathrm{~Hz} \mathrm{HX}-554$ transmissions at ranges of 500 and $1000 \mathrm{~km}$ with source depth of $350 \mathrm{~m} \ldots \ldots \ldots 119$ 


\section{Chapter 1}

\section{Introduction}

The science of sound propagation in the ocean has been developed extensively during the last few decades in response to practical needs. By now acoustic theory and simulation capabilities are well developed and can provide some general understanding and description of how sound travels in the ocean. However, there are still difficulties which limit accuracy. First of all, assuming that we know sound speed or the statistics of sound speed in the ocean precisely, there are still analytical and computational difficulties of calculating the sound pressure field and its statistics. The computational and theoretical ocean acoustics communities are still addressing these problems. A second issue is the lack of the adequate information about the sound speed field in the ocean as a function of position and time.

The rest of this chapter introduces the research questions addressed by this thesis, which is mainly about quantitatively understanding the limits that ocean randomness imposes on the practical uses of wave propagation. As a starting point, the following section introduces the background of long-range deep ocean acoustics. The second section describes the previous work and motivation for this thesis research work. Finally, the last section states the specific research objectives and outlines the remainder of the thesis.

\subsection{Background}

Acoustic propagation in the ocean is a field of a more general branch of science; namely, wave propagation through random media (WPRM). This research area is still very active with many open questions. During the 1960's, work on fluctuations in sound transmission through the ocean was a largely misguided attempt to graft the concept of wave propaga- 
tion through homogeneous, isotropic turbulence (HIT)[58] onto ocean variability. By the mid-1970's oceanographers had identified internal waves as the most important source of variability for the case of sound propagation through the ocean. Internal wave variability differ from HIT in several important aspects: first, the ocean environment is highly anisotropic, having vertical scales from $10 \mathrm{~m}$ to $1 \mathrm{~km}$, and horizontal scales of $200 \mathrm{~m}$ to 20 $\mathrm{km}$. Second, the connection between its spatial and temporal behavior is governed by the internal-wave dispersion relation rather than the usual HIT assumption. Third, the power law of its wavenumber $(k)$ spectrum is approximately $k^{-2}$ rather than $k^{-5 / 3}$. Fourth, its strength is a strong function of ocean depth. Beyond all the aforementioned differences, an even more unusual aspect is that, even in the absence of fluctuation, a ray from a source to a receiver is not a straight line; it is a curve controlled by a deterministic, depth-dependent, background variation in wave speed called the ocean sound channel[58, 40].

This new medium provided a challenge to those interested in WPRM as applied to ocean acoustics. A significant response to this challenge was developed over the late 1970's by a group of scientists, whose work as of 1978 is summarized in Flatté et al 1980[58]. But all those results are applied only to relatively high acoustic frequency and short-range experiments. In the late of 1980 's, low-frequency basin-scale experiments were motivated by the desire to measure ocean climate change, and scientists put a great amount of effort into extrapolating the established WPRM ideas to low-frequency and long-range sound transmission. In general, the research on acoustic wave propagation through the random deep ocean is a twofold problem which includes two interrelated topics: sound propagation and random media. It is safe to say that absolutely no progress has been made in any field of wave propagation through random media in which the researchers did not have a very strong understanding of the dynamics of the random media. Any better understanding of either topic will be very helpful to better understand the other one.

\subsection{Context and Motivation for this Study}

\subsubsection{Acoustic Fluctuations}

The history of low-frequency, long-range propagation experimentation can be traced back to 1944 in the last century[37], when the deep sound channel was discovered. Experiments up to the mid-1970's covered a wide range of investigation, and were confined to examine the 
measurements of parameters in the sonar equation, such as: transmission loss, reverberation, and ambient noise[68]. In the 1970s, the main effort to understand ocean acoustic WPRM focused on relatively high acoustic frequency and short-range experiments using broadband explosive sources and narrowband transducers. A large amount of data addressing the spatial and temporal statistics of fluctuations were collected[34, 31, 35, 41, 86, 40, 43, 26].

To explain observed amplitude and phase fluctuations, early efforts applied various techniques borrowed from electromagnetic scattering theory to calculate acoustic scattering from sound-speed fluctuations associated with homogeneous, isotropic turbulence. As explained, these theories of wave propagation in random media are inadequate when applied to propagation in the ocean. With the realization in the early 1970s that the fluctuations in ocean sound speed were dominated by internal waves which were neither homogeneous nor isotropic, a significant step forward was made in predicting acoustic fluctuations. The first success in this area was achieved for weak fluctuations by Munk and Zachariasen[71], whose absolute calculations of variances in phase and log intensity from internal-wave effects were within a factor of two of the available experimental results. In addition to the improvement in understanding the ocean sound-speed fluctuation field, a key theoretical breakthrough was the application of path integral techniques pioneered by Dashen, Flatté, and colleagues which lead to the formulation of analytical expressions for quantities like pulse spread, travel-time bias, and coherence as a function of vertical, temporal, and horizontal separations. Comparisons of measurements with the theory of Flatté, Dashen, and colleagues have been made mostly for short-range (20 to $300 \mathrm{~km}$ ) and high acoustic frequency (400 $5000 \mathrm{~Hz})[31,78,75,24]$.

Beginning in 1989 a series of experiments, mostly in the North Pacific, were undertaken to study low-frequency (order $30-300 \mathrm{~Hz}$ ) acoustic fluctuation (SLICE89, AET, ATOC, Heard Island, etc) $[64,65,66,3,25,2,84,70]$.

In 1989, a 50-element, 3-km long vertical line array (VLA) was deployed in the eastern North Pacific to receive $250-\mathrm{Hz}$ broadband signals from a moored source $1000-\mathrm{km}$ away (SLICE89). In the SLICE89 experiment it was discovered that acoustic fluctuations were much stronger than previously predicted, especially for acoustic energy which traveled within a few hundred meters vertically from the sound-channel axis[25]. The Acoustic Thermometry of Ocean Climate (ATOC) Acoustic Engineering Test (AET) [22, 23, 83], conducted in November 1994 and also in the eastern Pacific, used 75- $\mathrm{Hz}$ broadband signals 
and transmitted to a 700-m long, 20-element VLA at a range of $3250-\mathrm{km}$. The results were qualitatively similar to SLICE89 and the AET signals also showed surprising vertical and temporal coherence for the early ray-like arrivals which were far in excess of the then currently predicted values of [44]. Furthermore, the AET showed pulse time spreading to be lower than predictions[22], and intensity fluctuations were slightly larger than predicted by weak fluctuation theory[71, 23, 22].

It has been shown that the strongest acoustic scattering occurs near a ray upper turning point (UTP)[58], so acoustic propagation of order 1000-km involves order 10 to 20 scattering events. Thus, it is important to study about the physics of one or perhaps a few scattering events, so as to better understand the aforementioned results from long-range experiments. Previous work on single UTP propagation has been entirely at frequencies of $1000-\mathrm{Hz}$ or more (MATE Experiment[35], AFAR Experiment[40]), and is not directly related to the low frequency cases[20].

In the AET experiment, during a six-day period, acoustic signals were transmitted to two autonomous vertical line arrays (AVLA) with different locations; one located approximately $87 \mathrm{~km}$ from the source and one located $3250 \mathrm{~km}$. The long-range $(3250 \mathrm{~km})$ transmission data has been analyzed and results are described in a series of papers[22, 23, 83]. For the 87 $\mathrm{km}$ transmission data (addressed in this thesis), the arrival pattern of these transmissions consists of two time resolved and identifiable wavefronts, one with an initially downwards ray angle and two lower turning points (LTP) and one UTP, to be referred to as ID -3 , and the other arrival with an initially upwards ray angle and two LTP's and two UTP's, to be referred to as ID +4 . Since acoustic scattering is most pronounced near the UTP these two arrivals provide a view into the fundamental scattering physics at the first two UTP's, which form the basis for a long range propagation theory. These two arrivals show quite weak acoustic fluctuations and thus they allow an investigation of weak fluctuation theory at this low frequency of $75 \mathrm{~Hz}$. The analysis of this short range transmission data, together with comparison with weak fluctuation theory and numerical simulation, will be one objective of this thesis.

The AET signals which were recorded at bottom mounted Navy SOSUS arrays showed surprising phenomena as well. Ray-like arrival patterns were observed at several arrays and these arrivals could be identified with ray paths predicted using any of several oceanographic databases. However, several of the identified arrivals were associated with caustics 
whose predicted deepest extension was several hundred meters above the SOSUS receiver. In general, it has been shown that the edges of the shadow zones of the wavefront are significantly extended in depth, and in time[19, 22, 28]. This extension of the shadow zone shows that the effect of scattering in long-range low-frequency ocean acoustic propagation is to introduce a significant bias into the wavefront intensity pattern; that is to say the acoustic fluctuations cannot be considered a zero mean effect superimposed upon an otherwise deterministic wavefront pattern.

Parabolic equation simulations using GM internal-wave sound-speed perturbations cannot explain the depth extension of these caustics[19, 81]. This phenomenon is apparently different than the depth extension at the pulse crescendo, and may be associated with acoustic bottom interactions near the receiver or ocean surface interactions. Another possible explanation is that since this acoustic energy preferentially samples the upper-ocean internal-wave field where the GM model is known to be inadequate, the effect may be caused by non-GM internal waves, or micro-frontal activity measured by Rudnick [39]in the North Pacific.

These zeroth order changes in the wavefront lead to ensonification of shadow zones, and are thus of critical important for ocean acoustic remote sensing. One of the primary scientific objectives of Long-range Ocean Acoustic Propagation Experiment (LOAPEX), which was conducted between 10 September and 10 October 2004, was to study the evolution, with distance, of the mean wavefront intensity patterns to better understand the roles of scattering and diffraction in general. LOAPEX provided low-frequency broadband acoustic transmissions to vertical hydrophone arrays covering most of the deep ocean water column, and to bottom mounted horizontal hydrophone arrays surrounding the Eastern North Pacific Ocean. Transmission paths to the vertical hydrophone arrays varied from 50 to 3200 $\mathrm{km}$ among seven stations on the main LOAPEX path. These distances provide the controlled range dependency. At each of these seven stations the LOAPEX acoustic source was suspended from the ship for typically one to two days. Two source depths were used at each of the seven stations, $350 \mathrm{~m}$ and either 500 , or $800 \mathrm{~m}$. One part of this thesis is a study of the range evolution of the mean intensity of the wave front for the LOAPEX off-axis source transmissions. The extension of the wavefront mean intensity pattern toward the on-axis finale region will give information about modal energy transfer from high to low modes.

In general, a few decades of experimental work have provided a great amount of ob- 
servation data, and some of them provided a good match to theory $[58,40,44,78]$ (These observations were made using either single hydrophone receivers or very limited vertical aperture arrays); some do not, especially in the long-range low-frequency sound transmission data. All these results indicate the need for more theoretical and observational work.

\subsubsection{Ocean Modeling}

On the other hand, as mentioned previously, the improvement of acoustic prediction models requires a correct ocean sound speed fluctuations model. In the 1970's the introduction of the Garrett-Munk (GM) $[45,46,71,47]$ internal wave spectrum to the problem of ocean acoustic wave propagation was a significant breakthrough. As matter of fact, almost all work to date in predicting low-frequency acoustic fluctuations in the ocean has utilized the GM internal-wave model and a number of acoustic propagation experiments have been successfully compared with fluctuation theory based on internal wave dominance[58].

However, in basin scale acoustic transmissions, several considerations demand that we examine other processes than GM internal waves. This will be one focus of this thesis, where we will analyze observation of sound speed variabilities from the North Pacific Acoustic Laboratory (NPAL) 98-99 experiment. From the acoustical point of view the ocean is extremely variable: meso-scale eddies, currents, internal waves, and small-scale turbulence perturb the horizontally stratified character of the sound velocity and cause spatial and temporal fluctuations of the propagating sound. Large eddies in the ocean are most frequently observed near intensive frontal currents, such as the Gulf Stream and the Kuroshio. Meso-scale eddies are also found in the open ocean. Basin scale transmission ranges involve many correlation lengths of the ocean meso-scale field, whose characteristic scale is of order $50 \mathrm{~km}$ in the temperate latitude open ocean. A typical temperature anomaly for an eddy is $1^{\circ} \mathrm{C}$ (or roughly $4.6 \mathrm{~m} / \mathrm{s}$ sound speed). Therefore the horizontal gradients of eddies are roughly the same as internal waves, which have characteristic horizontal scale of $10 \mathrm{~km}$ and temperature anomalies of roughly $0.2^{\circ} \mathrm{C}$.

The other feature of basin scale transmission that leads us to examine other models than the GM is that the sound can have significant upper ocean interaction. The GM model was based largely on measurements within or below the main thermocline (roughly 1500 to $400 \mathrm{~m}$ depth at temperate latitudes). In the upper ocean, several different mechanisms can contribute to sound speed variability. Examples are: internal tides, ocean finestructure or 
spice[56, 29], and inertial wave oscillations $[52,53,57]$.

The internal tides band is interesting because it can be quite energetic and it is quasideterministic, having contributions from coherent source regions (like the Hawaiian Ridge) and from the incoherent ambient background. The tidal contribution to the acoustic fluctuations has been mentioned in the literature, perhaps because of a superficial resemblance of the phase fluctuations to tidal records[67]. Thus internal tides might play a significant but not dominant role.

Ocean "spice", the phenomena that the temperature and salinity of patches of hot salty water and cold fresher water compensate to yield equal density but not equal sound speed, have been proposed as one scattering mechanisms. Recently calculations by Dzieciuch and Munk [29] suggest that spice scattering in the upper ocean can be at-least as strong as upper ocean internal wave scattering. The preliminary analysis of underway conductivitytemperature-depth (UCTD) observation during NPAL 2004 SPICE and LOAPEX cruise also shows the spice might induce a amount of scattering of the same order as internal waves in the upper ocean[61].

The near-inertial frequency wave oscillations are usually neglected, from an acoustics standpoint, because in the limit in which the frequency approaches the local Coriolis frequency the vertical displacement goes to zero. Nonetheless, if the isotherms are tilted (for example by an eddy) the near-inertial horizontal current will produce a sound speed effect. Furthermore, inertial waves are known for their large shear $(d u / d z)$ and, in fact, at the mixed layer and several hundreds of meters below, inertial frequency upper ocean shear may play a comparable role to internal wave induced sound speed fluctuations as a source of upper ocean acoustic scattering[15].

The oceanographic literature on deep ocean internal waves is vast, but generally of the consensus that in the lower and main thermocline the Garrett-Munk universal internal wave spectrum $[45,77]$ provides a zeroth order description of internal waves. Nevertheless, within several hundreds of meters of the ocean surface, it is equally clear that there is a failure of the GM model[73, 74]. Furthermore, in the upper ocean, non-GM effects could be important in describing the depth extension of caustics at the Navy SOSUS arrays and the axial energy infilling from an off-axis sound source, which can not be explained by the existing GM internal wave model. An upper-ocean internal-wave model is needed so that acoustic fluctuation predictions can be made. Currently, in the oceanographic literature 
there is no model like the GM model for the upper ocean[54], thus direct measurements are needed for the purpose of our acoustic propagation studies. Through observation, we hope to quantify the sound speed variance and spectra for each aforementioned process, and also the seasonality of spectra in the internal wave band.

\subsection{Approaches and Objectives}

This thesis work uses data collected in the North Pacific Ocean during the last 15 years under the program names of Acoustic Thermometry of Ocean Climate (ATOC) and NPAL). Although serious investigations of long-range ocean acoustic propagation began after World War II, a hallmark experiment was the Heard Island Feasibility Test[84, 1, 63]. In that test electronically generated acoustic signals were sent and coherently received at very long ranges. This successful result led to the ATOC demonstration[2]. The purpose of ATOC was to show that a small number of acoustic transmitters and receivers could adequately characterize variations in the heat content of an entire ocean basin. The intent is to demonstrate that travel-time tomography can be used to measure ocean temperature over ranges of 3,000 to $10,000 \mathrm{~km}$. When the formal ATOC program came to an end, the Office of Naval Research (ONR) began sponsorship of the NPAL. This program uses the acoustic source and receiver network established during ATOC to focus on basic research related to long-range acoustic propagation while at the same time allowing the continuation of the time series of climate related data.

This research consists of three parts:

The first objective is quantification of ocean sound speed variance and space-time scales in the NPAL 98-99 environmental data due to the internal wave continuum, the near inertial waves, the internal tides and the sub-inertial motions. In this effort, the validity of Garrett \& Munk (GM) internal wave model in the upper ocean of North Pacific, will be examined. This is needed to assume that propagation theories based on this model are valid, or to determine whether adjustment need to be made.

The second objective is to test the application of Rytov weak fluctuation theory as modified by Munk and Zachariasen (MZ)[71] in this case of low-frequency sound propagating through internal waves in the deep ocean. As we know, an important WPRM issue is to delineate the general regimes of acoustic wave propagation in the ocean as described by 
fully saturated, partially saturated, and unsaturated propagation. Data has been analyzed at long ranges and shows well developed fluctuations. At short ranges where fluctuations should be weak, we have no experimental results. In this thesis, statistics of low-frequency, short-range acoustic transmission in the North Pacific Ocean are presented for the first time. It has been shown that the strongest acoustic scattering occurs near a ray UTP, so long range acoustic propagation involves multiple scattering events. So our general goal is to understand the physics of one or two scattering events, so as to better understand the long-range experiments. The basic physics of the $M Z$ model that is to be tested is that there is weak, single forward scattering, and that there is a resonance condition between the sloping ray path and the internal waves whose crests are aligned with the sloping ray.

Third, data from the recent LOAPEX are presented, analyzed and compared to simulations. For the off-axis source, ocean sound speed fluctuations cause an in-filling of acoustic energy into the finale region. The simplest acoustic observable associated with these effects is the mean intensity, a second moment. In this thesis we aim to understand the following questions quantitatively: Does high angle acoustic energy from an off-axis source transfer energy to low angles in the axial region of the waveguide? What are the relative contributions from diffraction and scattering for finale region arrivals? How does this energy transfer scale with range?

The organization of this thesis is as follows. In chapter 2, we first start to quantify the ocean sound speed space-time scales due to internal waves continuum, near inertial waves, internal tides and sub-inertial motions from the NPAL 98 -99 environmental data. In Chapter 3, we analyze the space-time scales of acoustic fluctuations in the weak fluctuations regime, i.e. from observation of $75-\mathrm{Hz}$, broadband transmissions to $87-\mathrm{km}$ range in the AET experiment. In chapter 4 , we study the evolution of the mean intensity wavefront patterns for off-axis source propagation at different range of LOAPEX data. Finally, Chapter 5 summarizes the thesis contribution and indicates directions for future research. 


\section{Chapter 2}

\section{Observations of Deep Water,}

\section{Upper Ocean Sound Speed}

\section{Structure in the Eastern North}

\section{Pacific Ocean}

\subsection{Introduction}

From September 1998 through July 1999 the NPAL group performed an integrated acoustics and oceanographic experiment in which sound transmitted from the ATOC bottom mounted source off the island of Kauai was received on a series of five closely spaced vertical arrays (billboard array) on Sur Ridge off Monterey, California[85]. To aid in the interpretation of the observed acoustic variability, a significant oceanographic component was involved in the NPAL 98-99 field year, in which moored and shipboard observations of temperature and salinity structure in the upper $800-\mathrm{m}$ of the ocean were obtained. The central scientific goal is defining the space time scales of the main thermocline and upper ocean sound speed structure caused by mesoscale eddies, internal tides, internal waves, and other fine scale processes. In the deep ocean, sound speed fluctuations are generally associated with the vertical advection of local mean sound speed structure and can thus be represented by

$$
\delta c_{\zeta}=\left(\frac{d c}{d z}\right)_{p} \zeta
$$


where $\zeta$ is the mesoscale or internal wave vertical displacement of a density surface (assumed adiabatic) and $(d c / d z)_{p}$ is the mean gradient of potential sound speed. In addition to sound speed structure caused by vertical displacements there can also be sound speed structure along surfaces of constant density due to differing water mass co-mingling (temperature and salinity). This sound speed effect is often termed ocean finestructure or spice[55, 39, 29]. Oceanographic data from the NPAL 98-99 field year cannot address the relative contributions of displacement and spice because of the stringent requirements of salinity resolution needed for such a separation.

The oceanographic literature on deep ocean internal waves is vast (GM, Cairns and Williams, IWEX, Munk81, Pinkel, etc) $[45,8,6,77,73]$ but generally of the consensus that in the very deep, and in the main thermocline, the Garrett-Munk (GM) universal internal wave spectrum[46, 77] provides a zeroth order description of internal waves. Recent work by Levine (2002)[54] has also improved upon the GM model for low-frequency internal waves in the semi-diurnal to inertial frequency band. In the upper ocean, within several hundreds of meters of the ocean surface, it is equally clear that there is a failure of the GM model[73, 74]. At issue here is 1) the factorization of the GM spectrum in terms of frequency and mode number, 2) the vertical asymmetry of internal wave energy propagation near inertial frequencies, and 3) the random phase approximation of the various wavenumber components. The degree at which these issues with the GM model arise appears to be rather geographically dependent (non-universal), and thus direct measurements are needed for the purpose of our acoustic propagation studies.

The basic outline of this chapter is as follows. Section two gives a description of the sound speed observations taken during the NPAL 98-99 field year. Section three uses the moored observations to address the question of the partitioning of sound speed variance between mesoscale and internal wave frequency bands. Within the internal wave band the seasonal changes of sound speed variance as a function of depth are given, and estimates of the relative contributions between random and deterministic internal tides is addressed. The sound speed variance derived from XBT measurement is also presented in this section. Section four examines frequency, vertical, and horizontal spectra of sound speed and temperature and compares the results to the GM internal wave model. Section five has summary and conclusions. 


\subsection{The Experiment}

The observations of ocean sound speed structure and current presented in this paper were obtained in the Eastern North Pacific Ocean along an acoustic transmission path between the Island of Kauai and Sur Ridge off Monterey California, USA (See Figure 2-1). The acoustic path was part of the NPAL 1998-1999 field year in which $75 \mathrm{~Hz}$ broadband signals were transmitted from a bottom mounted source off of Kauai to a billboard receiving array $3500 \mathrm{~km}$ distant on Sur Ridge. During early transmission to the billboard array, a hydrographic cruise along the path was conducted August 15-30, 1989. During this cruise Conductivity, Temperature, and Depth(CTD), and eXpendable BathyThermograph (XBT) measurements were made at various horizontal and vertical resolutions. In addition to hydrographic measurements, two well separated moorings were installed to measure temperature, salinity, and pressure in the upper $800-\mathrm{m}$ of the ocean for a year duration (Figure 2-1,2-2). The moorings were also equipped with $300 \mathrm{kHz}$ Acoustic Doppler Current Profilers (ADCP). Roughly a year later, an identical hydrographic cruise was carried out June 19 to July 3, 1999, and the moorings were recovered. The moored data, and the shipboard CTD and XBT observations form the basic data sets to be presented in this chapter, and their detailed description is given next.

\subsubsection{Moored Observations}

Figure 2-2 shows a diagram of the eastern mooring configuration of sensors. The instrumentation consisted of upward and downward looking $300 \mathrm{kHz}$ ADCP, 6 CTD units, and 10 temperature only units. The western mooring had the identical suite of instrumentation except there was only one ADCP in the upward looking configuration. The western mooring was located at $28^{\circ} 14.14^{\prime} \mathrm{N}, 148^{\circ} 14.57^{\prime} \mathrm{W}$ with a water depth of $5335 \mathrm{~m}$, and east mooring was located at $33^{\circ} 30.09^{\prime} \mathrm{N}, 133^{\circ} 58.65^{\prime} \mathrm{W}$ with a water depth of $5003 \mathrm{~m}$.

All instruments were configured to measure water properties over an entire year, so the ADCP's ensemble averaged over 20 minute intervals, and utilized range bins of $4-\mathrm{m}$. The CTD instruments recorded every $300 \mathrm{~s}$, and the temperature only instruments recorded at a slightly higher rate of one sample every $210 \mathrm{~s}$. The nominal sensor spacing was $30-\mathrm{m}$. For subsequent analysis the temperature only records were interpolated onto the CTD recording interval (5 minutes), and salinity values were interpolated in depth to give values where only 

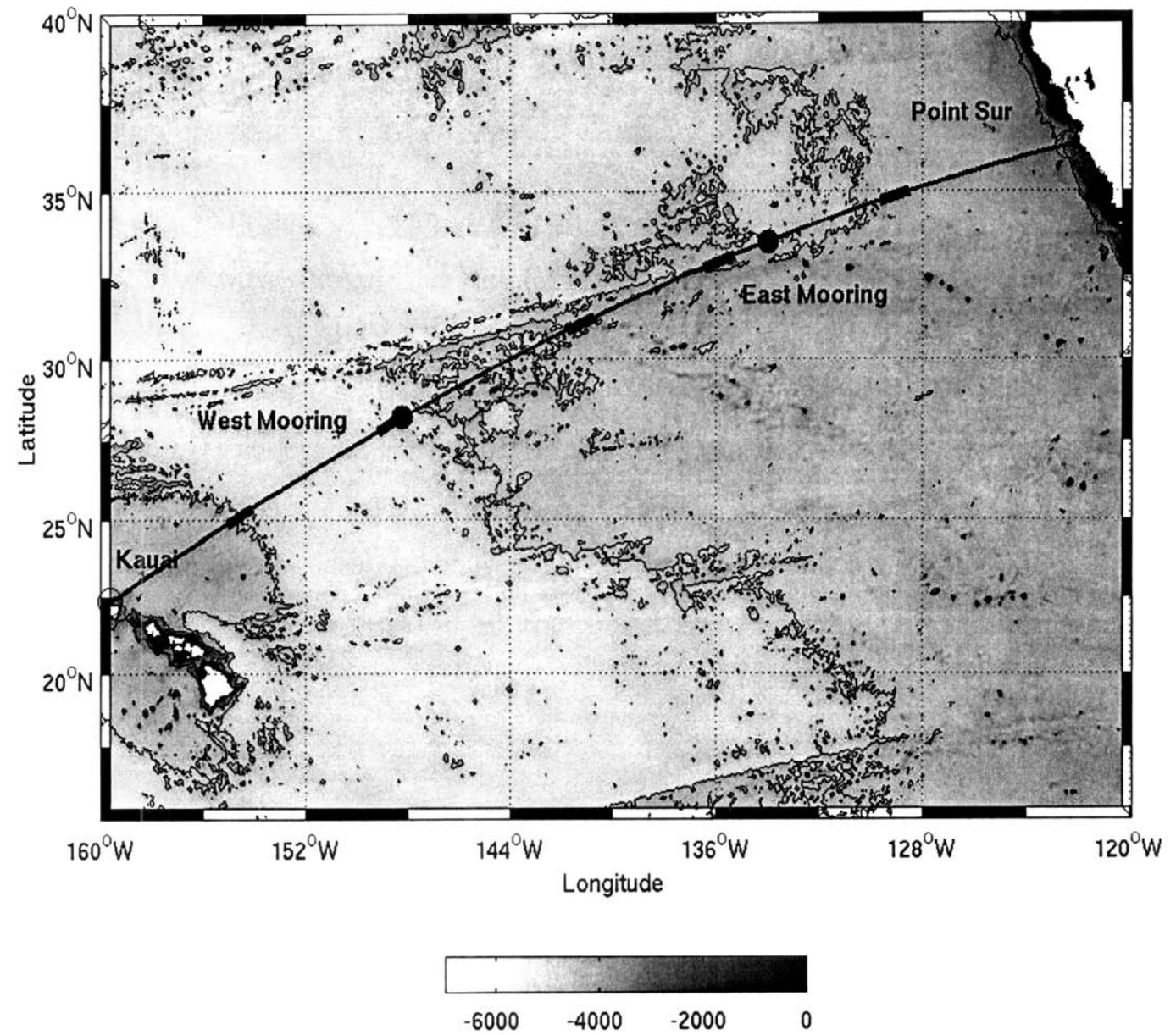

Figure 2-1: NPAL 98 - 99 acoustic transmission path between the Kausi Source and Sur Ridge receiver with North Pacific ocean bathymetry map superimposed. West and East environmental moorings are denoted by two dark round dots. The five pieces of broad dark line along the transmission path indicate the region with the high resolution XBT survey. The colorbar indicates the depth range. 


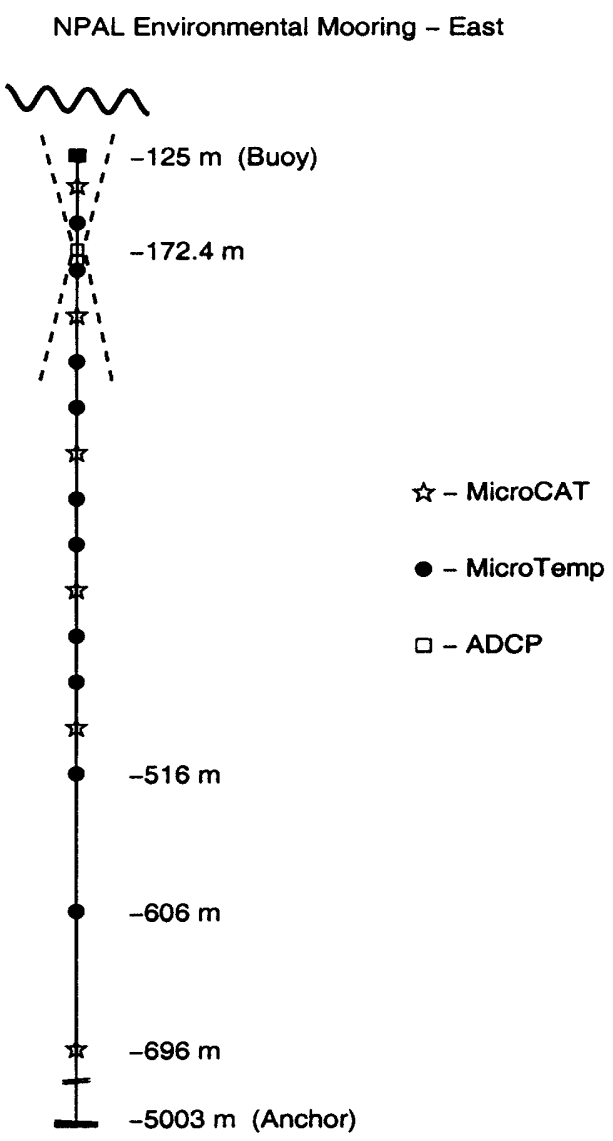

Figure 2-2: The diagram of eastern mooring configuration of sensors. The East Mooring is located at $3330.0936 \mathrm{~N}, 13358.6451 \mathrm{~W}$ with a water depth of $5003 \mathrm{~m}$. The buoy depth is about $125 \mathrm{~m}$. The dash line denotes the fans of the upward/downward looking ADCPs. The western mooring is located at $2814.140 \mathrm{~N}, 14814.566 \mathrm{~W}$ with a water depth $5335 \mathrm{~m}$, which had the identical suite of instrumentation except there was only one upward looking ADCP installed. 
a temperature measurement was made. Because of mooring motion, and resultant shifts in depth the records were further interpolated onto a uniform set of depths for Eulerian analysis: The reference depths were uniformly spaced at $30 \mathrm{~m}$ steps starting at $160 \mathrm{~m}$ and ending at $640 \mathrm{~m}$. Sound speeds were computed from temperture, salinity and depth using the Chen and Millero's formula. Figure 2-3 shows a several day timeseries of a few of the temperature and salinity records from the eastern mooring to demonstrate the quality of the data.

\subsubsection{XBT Surveys}

During the deployment and recovery cruises two types of XBT surveys were carried out. For the purpose of mesoscale mapping, 750-m depth XBTs were dropped every 30-km along the entire sound transmission path. Figure 2-4 shows the two mesoscale resolution maps from the 750-m XBTs, and a relatively weak mesoscale field is evident, except for a few eddies near the island of Kauai. For the purpose of small scale temperature mapping each cruise had 5 "high resolution" XBT surveys in which calibrated 450-m depth XBTs were dropped every $1.5 \mathrm{~km}$ for roughly a $120-\mathrm{km}$ range. Figure $2-5$ shows a few of these high resolution surveys, and the significant small scale structure that exists.

\subsubsection{CTD Data}

Both cruises obtained CTD data along the transmission path at roughly $150-\mathrm{km}$ resolution. Every third CTD cast was a full water depth cast, while the other two were taken to $1500 \mathrm{~m}$ depth (Dickinson, Howe, and Colosi 2003). The CTD data provide important estimates of sound speed and buoyancy frequency profiles, for the subsequent analysis of this paper. Figure 2-6 shows the variation of the buoyancy frequency profiles along the transect.

\subsection{Strength of Sound Speed Fluctuations}

Of fundamental importance to ocean sound propagation is the strength of the sound speed fluctuations that are superimposed on the background waveguide. The year long moored records allow a separation of sound speed fluctuations based on the various ocean processes with different timescales. 

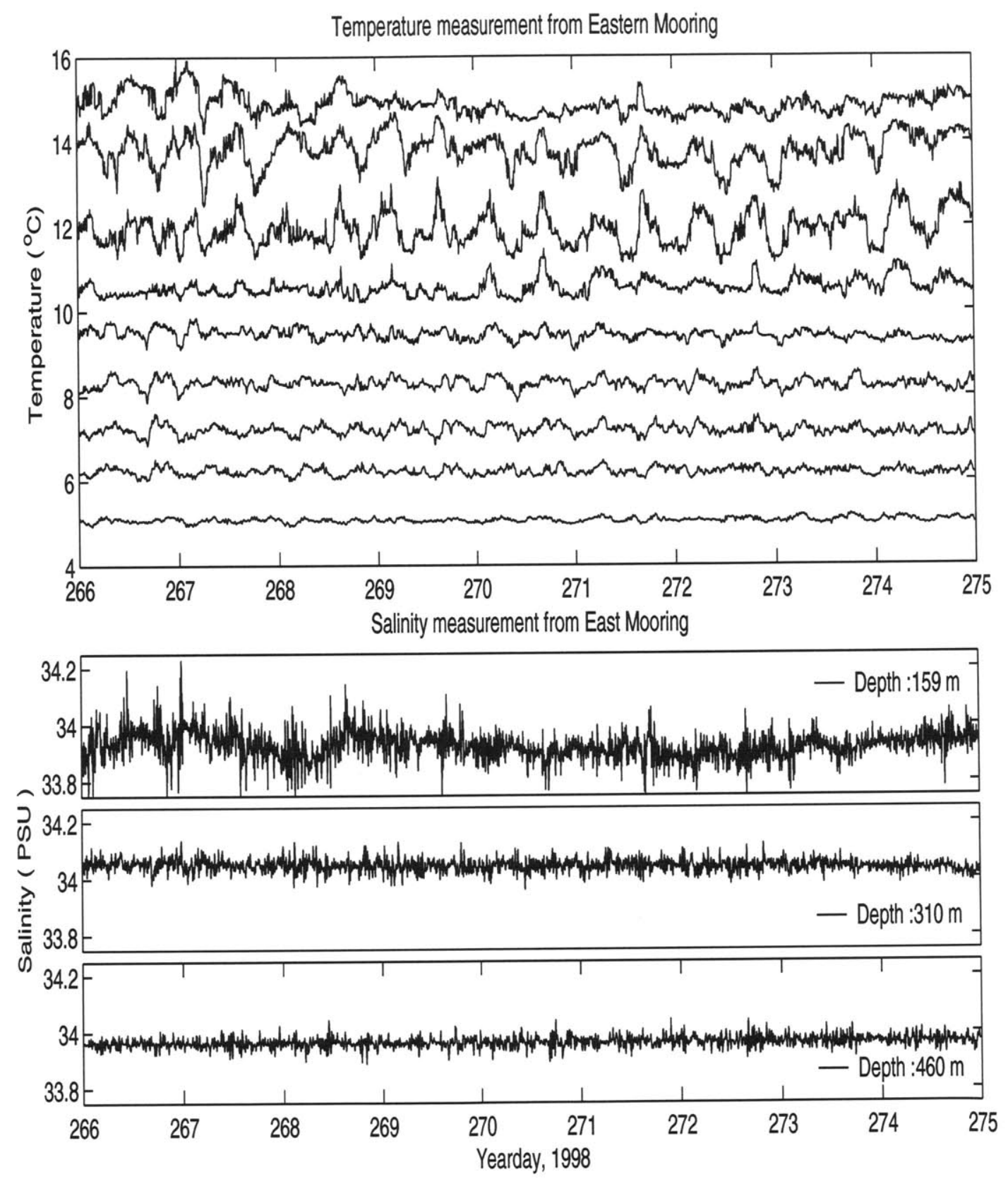

Figure 2-3: A 10-day timeseries of temperature (upper panel) and salinity (lower panel) records from eastern mooring. The depths displayed in the lower panel are mean depths calculated from the pressure measurement. The temperature measurement are collected at the depth from about $128 \mathrm{~m}$ to $580 \mathrm{~m}$, with warm water near surface and cold water in the deep. 

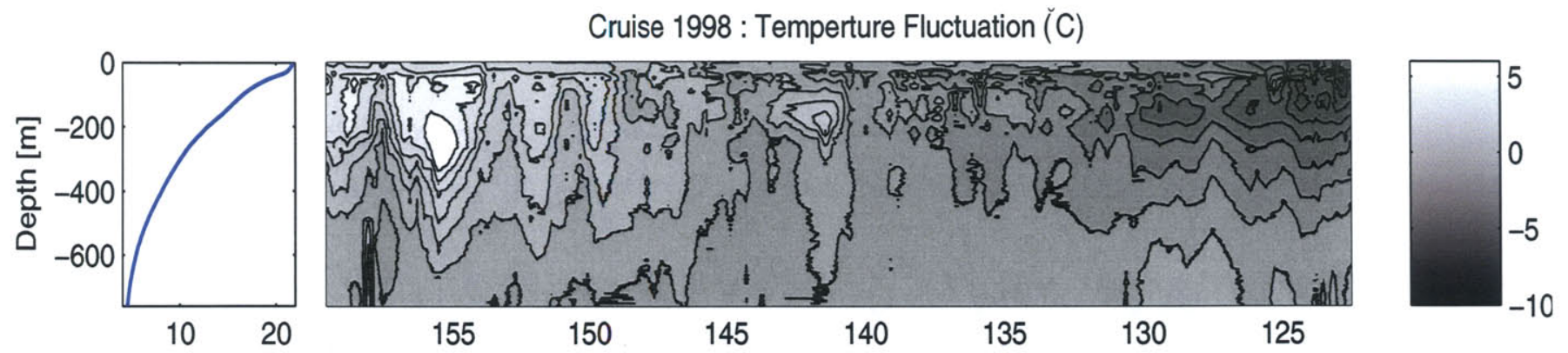

$\left({ }^{\circ} \mathrm{C}\right)$
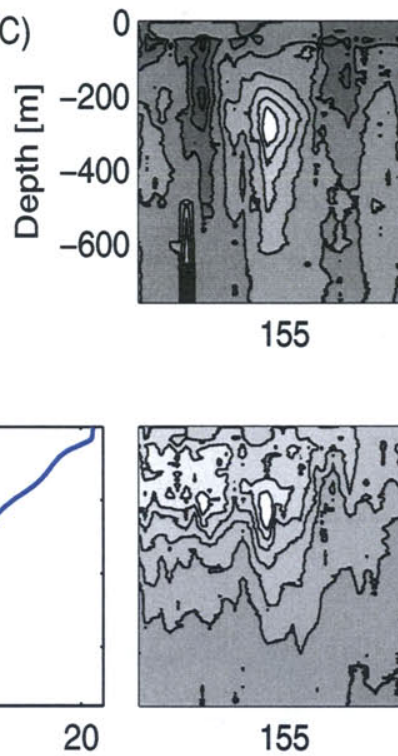

155

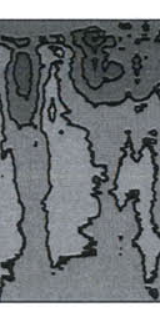

135

130

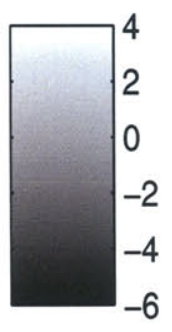

$150 \quad 145$

\begin{abstract}
140
\end{abstract}
130

Cruise 1999 : Temperture Fluctuation (C)
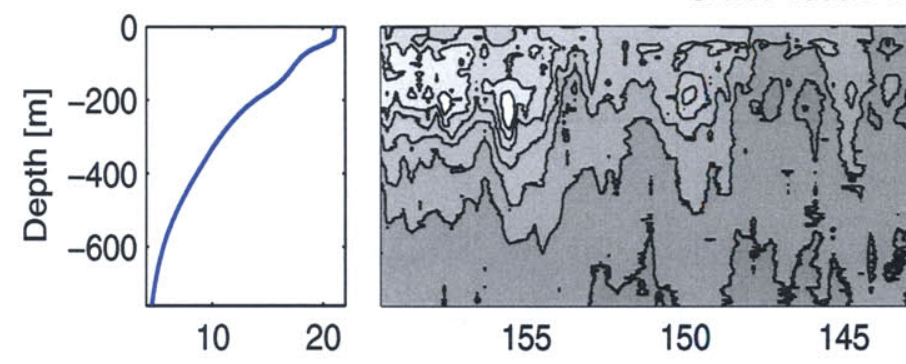

$145 \quad 140$
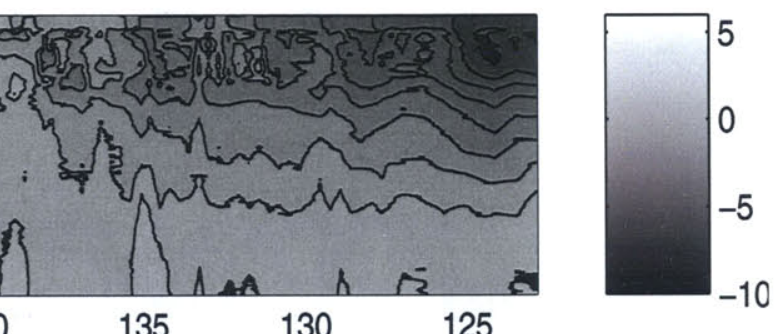

$\left({ }^{\circ} \mathrm{C}\right)$
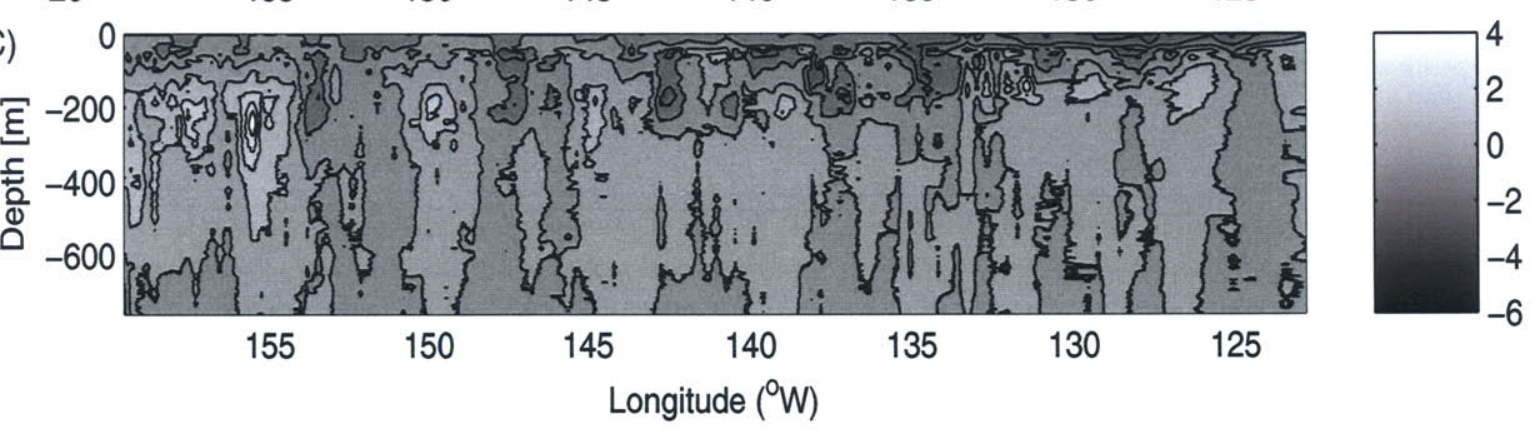

Figure 2-4: A 750-m depth XBT survey for the purpose of mesoscale mapping during the IW98 and IW99 cruises. The upper three panels correspond to the IW98 cruise measurement, the lower three panels correspond to the IW99 cruise measurement. For each cruise, the left panel shows the mean temperature profile, the right upper panel shows temperature fluctuation with mean profiles removed, and the right lower panel shows the temperature with the climatological values removed. A few warm eddies near the island of Kauai emerges in the left region of the right panel. 

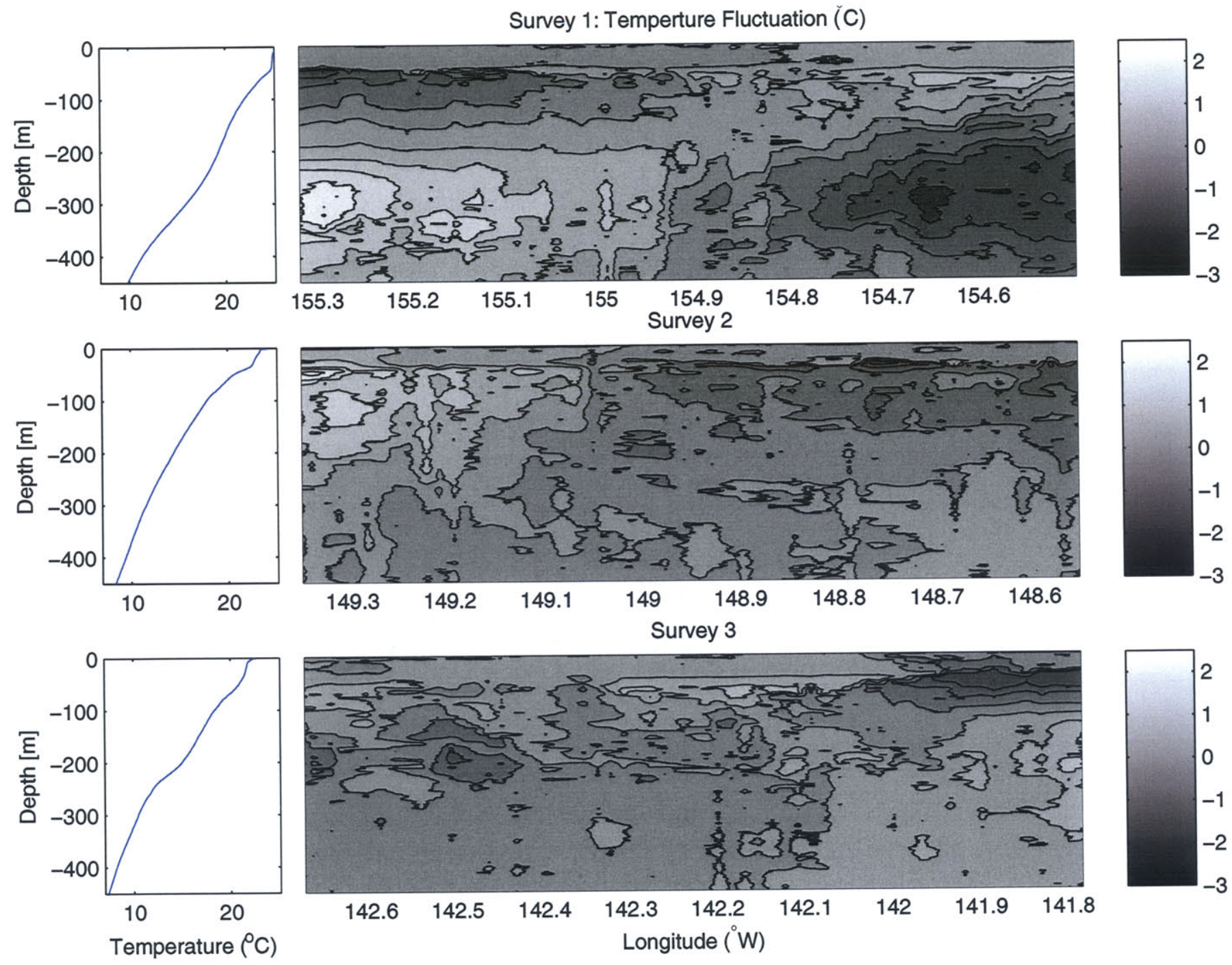

Figure 2-5: The first three "high resolution" XBT surveys during the IW98 cruise. The left panels denotes the mean temperature profiles, the right panels are the temperature fluctuations with mean profiles removed. The cruise started from the Kauai island with heading to the north-eastern. The mean profile shows the temperature decreasing as the ship went to north. Note that the striking contrast in the Survey 1 with cold water in the north east and warm water in the south west. 


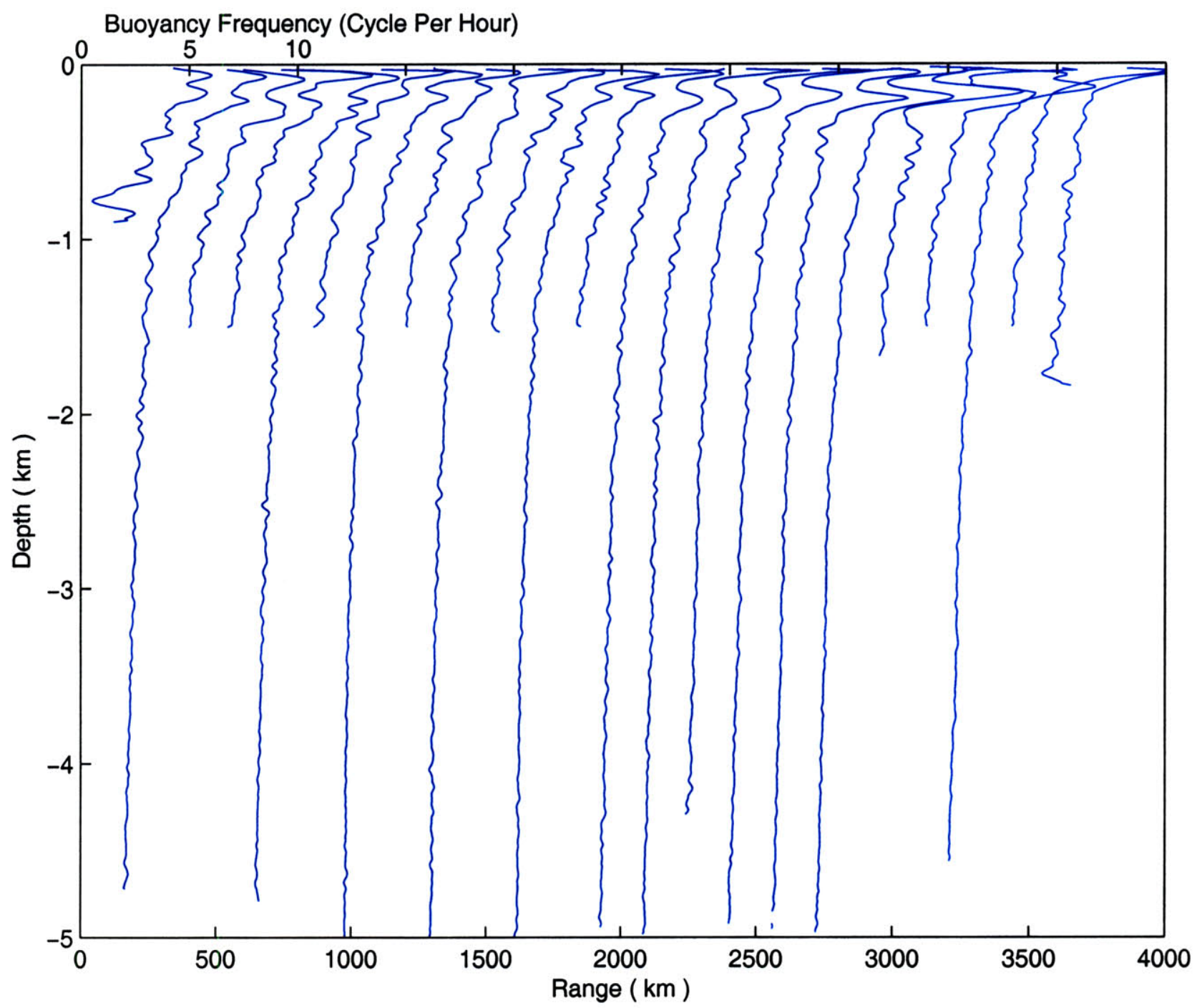

Figure 2-6: The buoyancy frequency profiles as a function of range between the source and receiver, derived from the CTD casts along the transect during the IW99 cruise. The CTD was deployed along the transmission path at roughly $150-\mathrm{km}$. The profiles are plotted with a buoyancy frequency offset of 2 cycle per hour. The lower $\mathrm{X}$-axis roughly denotes the range from the source to the receiver. 


\subsubsection{Internal Wave and Mesoscale Frequency Bands}

The first separation is based on the local Coriolis parameter $f$ and we denote sound speed fluctuations in the bands greater-than/less-than $f$ as the internal wave and mesoscale bands, respectively. Figure 2-7 shows an example of a frequency spectrum of sound speed fluctuations from the East mooring, where the internal wave and mesoscale bands are shown: There is a clear spectral gap at frequencies just below the inertial frequency (the kinematical internal wave cutoff frequency), followed by a rise in low-frequency energy in the mesoscale band. For a given depth the sound speed variances in the internal wave and mesoscale bands are computed as follows: for the internal wave band, the year long timeseries is high pass filtered using a cutoff frequency of $f / 3$ and the variance is computed from the resulting timeseries; for the mesoscale band the timeseries is low-pass filtered with a cutoff frequency of $f$ and the variance is computed from the resulting de-meaned timeseries. An example of this processing procedure is displayed in Figure 2-8 with original time series data, internal wave band data and mesoscale frequency band data in the upper,central and lower panels, respectively. Figure 2-9 shows the resulting internal wave and mesoscale sound speed variances as a function of depth for the East and West moorings. The mesoscale fluctuation is clearly more energetic than the internal wave band fluctuation. The maximum mesoscale fluctuation is of order $4 \mathrm{~m} / \mathrm{s} \mathrm{rms}$ or roughly $1^{\circ} \mathrm{C} \mathrm{rms}$ in temperature. This represents a rather weak mesoscale field as is known to be the case in the Eastern North Pacific Ocean. The internal wave band has a maximum fluctuation of $1-1.5 \mathrm{~m} / \mathrm{s} \mathrm{rms}$ with significantly smaller values at depth.

\subsubsection{Seasonal Variation in the Internal Waves Band}

Seasonal variations of internal wave band fluctuations are of great interest since winter storm forcing can have some effect on internal wave levels, especially near the inertial frequency. Figure 2-10 shows the break down of internal wave variance by season for the east and west moorings: Summer is September 1, 1998 - September 30, 1998, Fall is October 1, 1998 to December 31, 1998, etc.... For the East mooring a slight seasonal dependence is evident with summer showing the smaller variation. For the West mooring, however, the seasonal dependence is much more pronounced with summer and fall showing much reduced variation over winter and spring. 


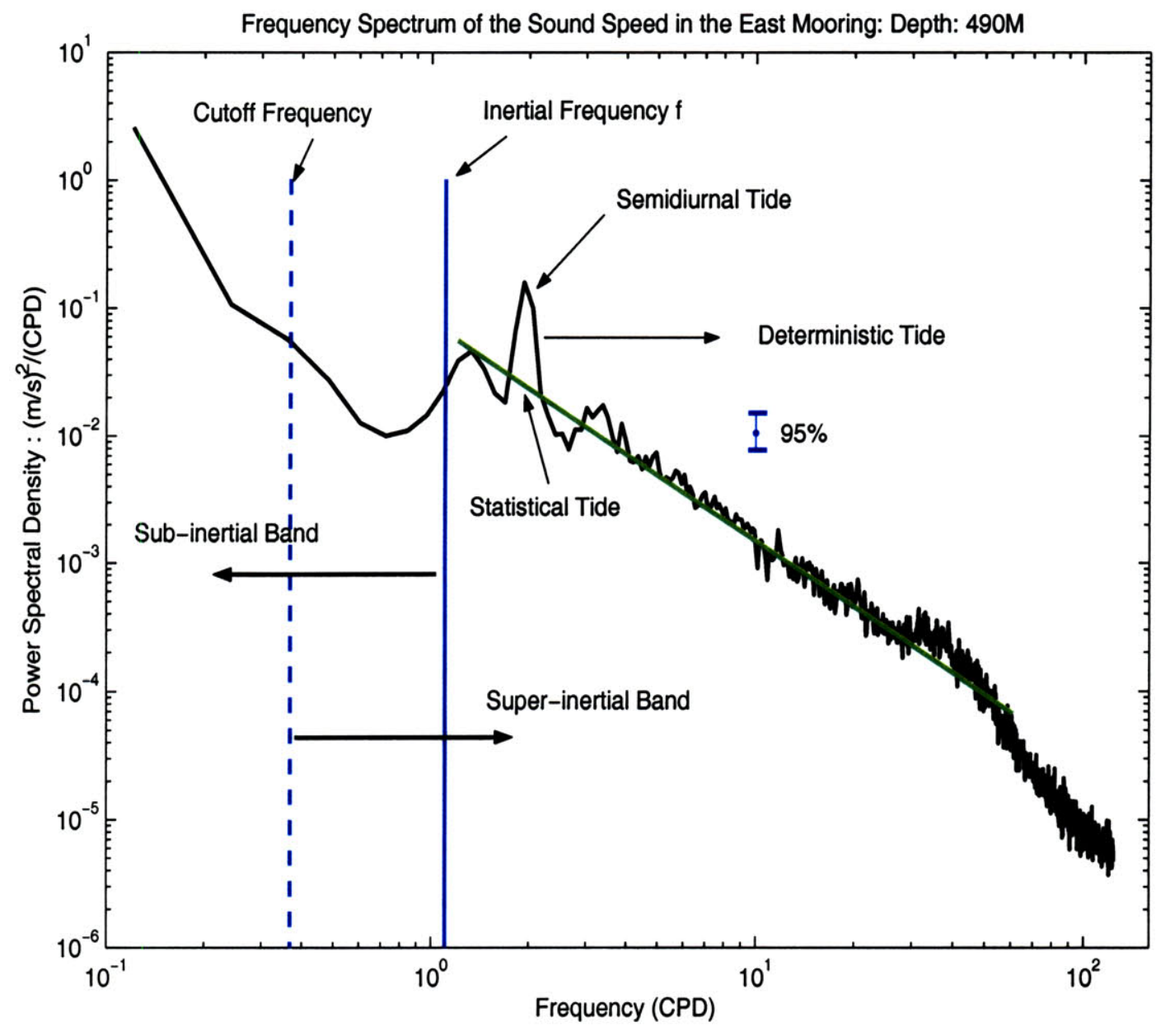

Figure 2-7: An example of frequency spectrum of the sound speed in the East Mooring at the depth $490 \mathrm{~m}$. This figure explains the separation of the mesoscale frequency and the internal wave bands, which is based on less-than the local Coriolis frequency $f$ (the solid vertical straight line)or greater-than $1 / 3 f$ (i.e. the Cutoff frequency showing in the dash vertical straight line). The significant peak denotes the semidiurnal tide. The separation of coherent(deterministic tide) and incoherent(statistical tide)part of semidiurnal tide is shown in this figure, which is based on extension of the power law continuum from high frequency. The deterministic tide component is the energy above the background continuum[21]. 
Time Series of Sound Speed in the East Mooring: Depth $490 \mathrm{~m}$
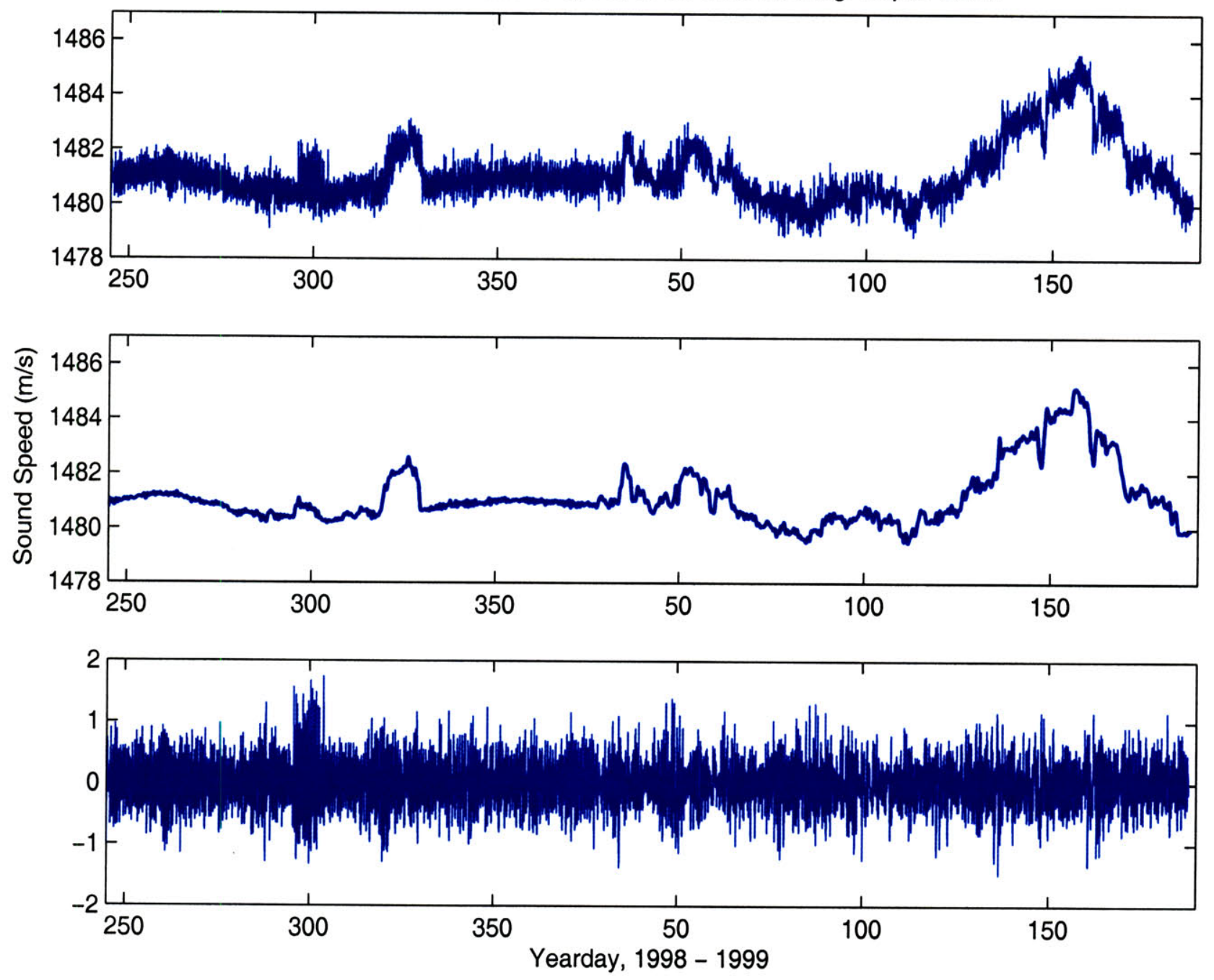

Figure 2-8: An example of separating the internal wave and mesoscale frequency bands from the original time series (upper panel). A fourth order Butterworth digital high pass filter is applied to the time series with cutoff frequency at $1 / 3$ of the local Coriolis frequency, the output is the internal wave band sound speed fluctuation, which is shown in the lower panel. The mesoscale frequency bands data (central panel)is obtained by applying the low-pass filter to the original data at the cutoff frequency of local Coriolis Frequency $f$. 

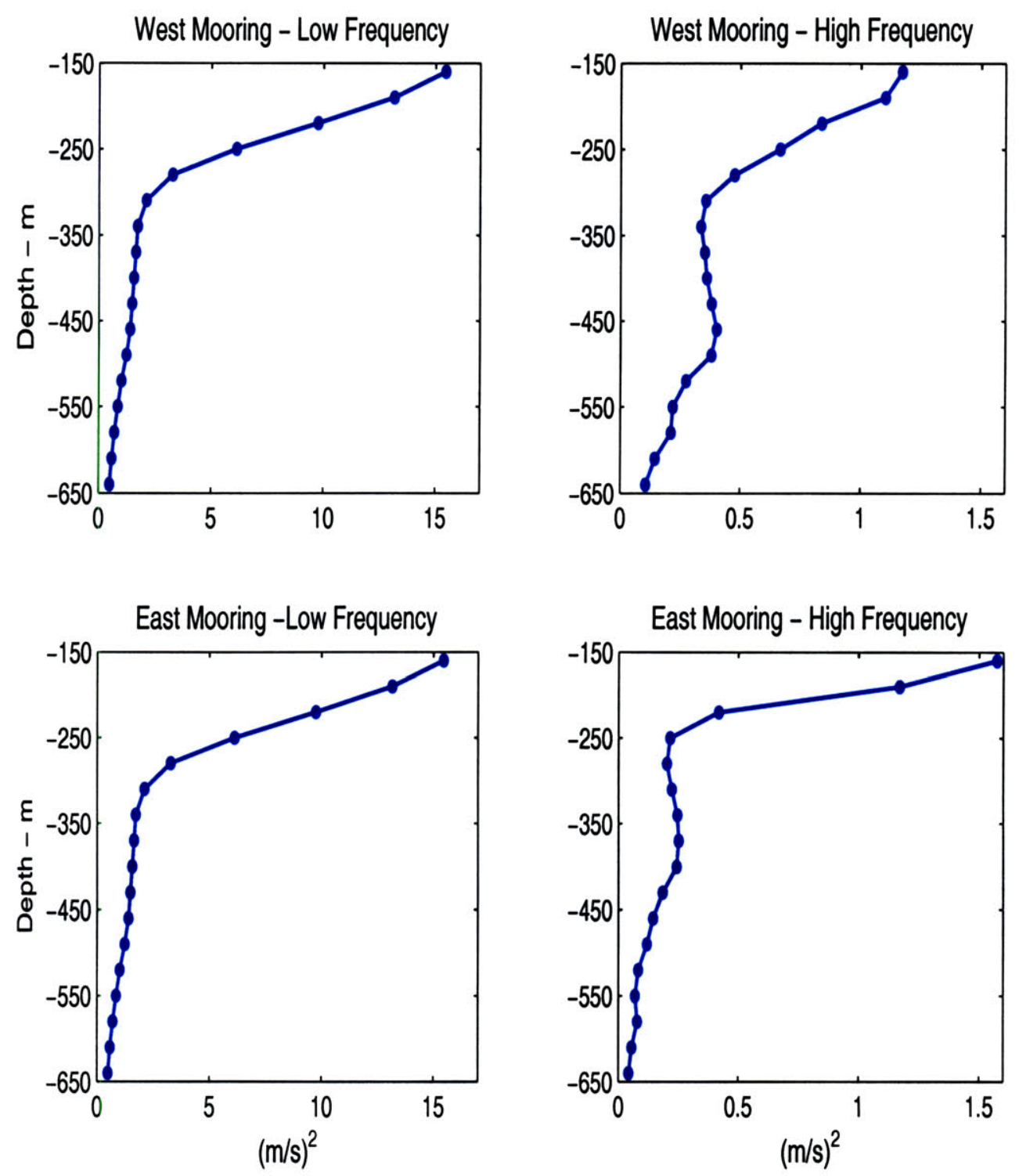

Figure 2-9: The internal wave and mesoscale sounds speed variance as a function of depth over the whole mooring deployment. The left two panels shows the mesoscale frequency band, the right two panels shows the internal wave band for both west and east moorings. The mesoscale fluctuation is clearly more energitic than the internal wave band fluctuation. 

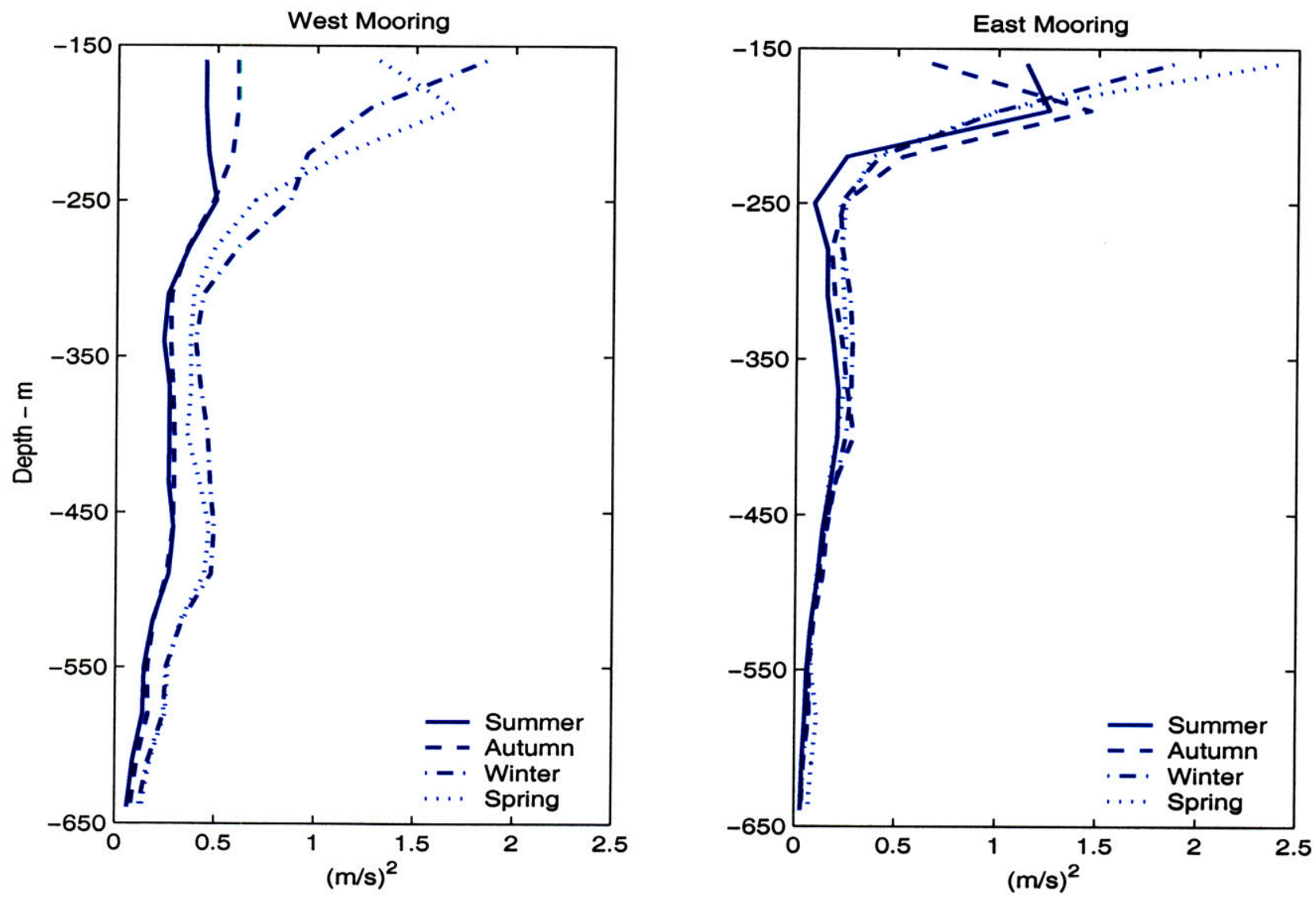

Figure 2-10: The seasonal variation of the sound speed variance as function of depth in the super-inertial band: left panel - the eastern mooring; right panel - the western mooring. Summer is September 1,1998- September 30,1998, Fall is October 1,1998 to December 31,1998, Winter is January 1,1999 to March 31,1999, and Spring is April 1,1999 to June 22,1999 . The seasonal variation is much more pronounced in the west mooring than in the east mooring. 


\subsubsection{Internal Wave Band Model Comparisons}

A comparison with a canonical model is in order here and such a model has been described by Munk (1976)[71], which gives,

$$
\left\langle\delta c^{2}\right\rangle(z)=\left(\frac{c_{0} \mu}{g}\right)^{2} N_{0} N^{3}(z) \zeta_{0}^{2}=\left\langle\delta c_{0}^{2}\right\rangle \frac{N^{3}(z)}{N_{0}^{3}}
$$

where $\mu(z)$ represents the nominal temperature and salinity gradients, and is a function of $T$, $S, d T / d z$, and $d S / d z[58]$. Here, $\mu$ is set to $24.5 . g=9.8 \mathrm{~m} / \mathrm{s}^{2}$ is the acceleration of gravity, $c_{0}=1500 \mathrm{~m} / \mathrm{s}$ is the nominal speed of sound in sea water, $N(z)$ is the buoyancy frequency profile, $N_{0}=3 \mathrm{cph}$ is a reference buoyancy frequency, and $\zeta_{0}=7.3 \mathrm{~m}$ is a reference $\mathrm{rms}$ internal wave displacement at $N(z)=N_{0}$. Using the aforementioned parameters we find $\left\langle\delta c_{0}^{2}\right\rangle=0.55(\mathrm{~m} / \mathrm{s})^{2}$. Using CTD data from the deployment and recovery cruises we can compute $N(z)$ and thus $\left\langle\delta c^{2}\right\rangle(z)$ profiles for Fall 1998 and Spring 1999 to compare with the seasonally derived moored sound speed fluctuations. Figure 2-11 shows the comparison

with the canonical model. The shaded region represents a range of $\left(\mu^{2} \zeta_{0}^{2}\right)^{\frac{1}{2}}$ from 0.5 to 1.0 time the standard value of 24.5 , and for the eastern mooring in particular the lower value of 0.5 appears to be the most appropriate.

\subsubsection{Internal Tide Variability}

In Figure 2-7 a clear semidiurnal peak ( $\sim 2 \mathrm{cpd})$ is seen in the spectrum, and this energy is primarily due to internal tides though it is conceivable that some of the variability may be due to barotropic tidal currents advecting horizontal gradients of temperature and/or salinity. For the spectrum calculation in Figure 2-7 the data were interpolated onto nearly 5.822 minute samples to yield an integer number of samples in an $M_{2}$ tidal period (12.42 hours) and thus the semidiurnal spectral peak is essentially one bin wide. The total sound speed variance from the tide is then taken to be the variance in the one $M_{2}$ peak; this is shown in Figure 2-12. This variance is further divided into a coherent and incoherent part[21] based on the extension of the nearly $\omega^{-2}$ high frequency range of the spectrum (See Figure 2-7 ). The variability is dominated by the coherent part of the $M_{2}$ peak energy, but compared to the total internal wave band energy the $M_{2}$ energy is quite small. 

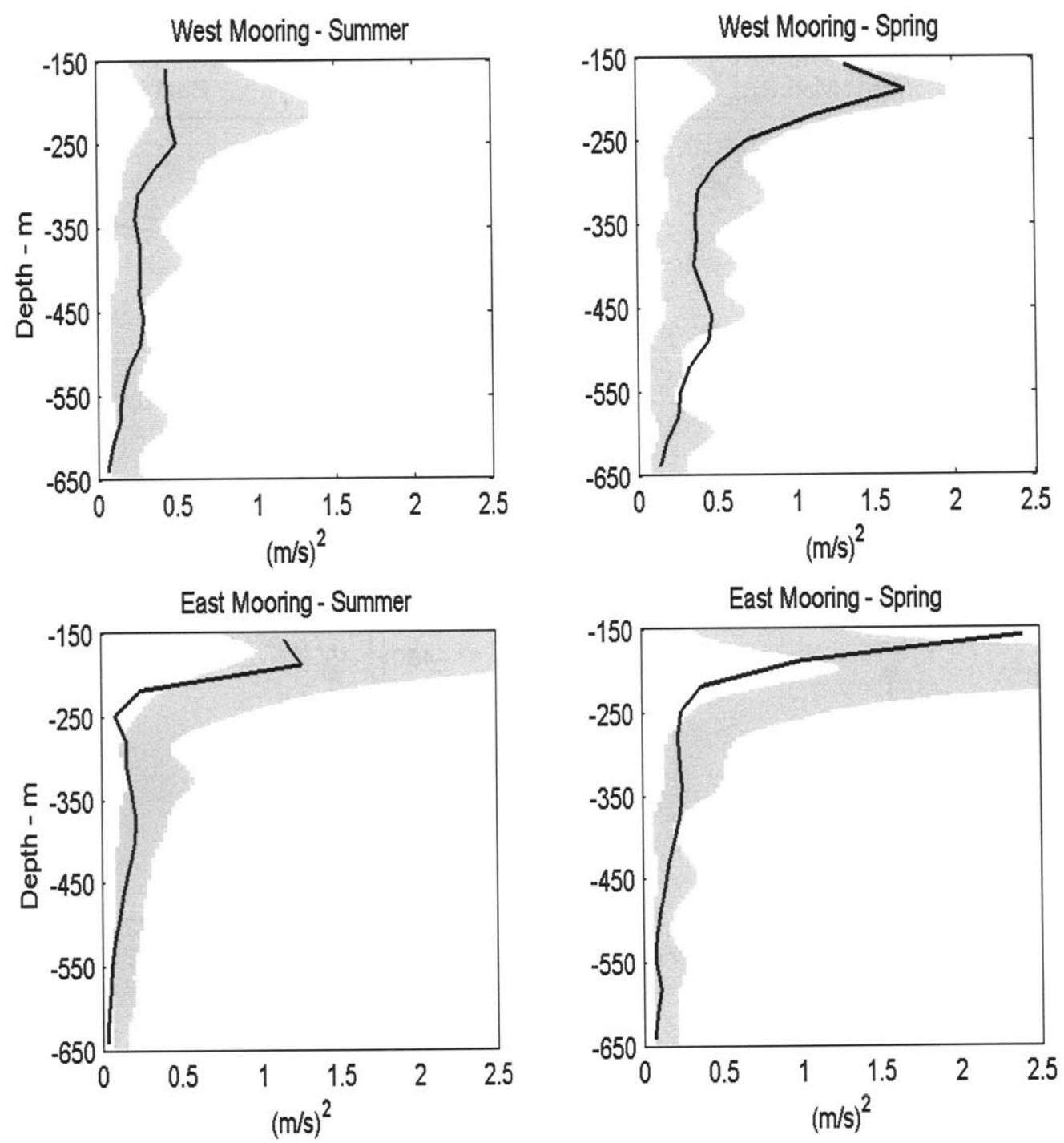

Figure 2-11: A comparison between the sound speed variance and canonical model, see Equation 3.26. The variance of sound speed in the season of summer and spring from the east and west mooring, are compared with the Munk $N^{3}$ scaling. The buoyancy frequency profiles are derived from CTD data collected during the IW98 and IW99 cruises which were selected near the mooring position. The shaded region represents a range of $\left(\mu^{2} \zeta_{0}^{2}\right)^{\frac{1}{2}}$ from 0.5 to 1.0 time the standard value of 24.5 . 

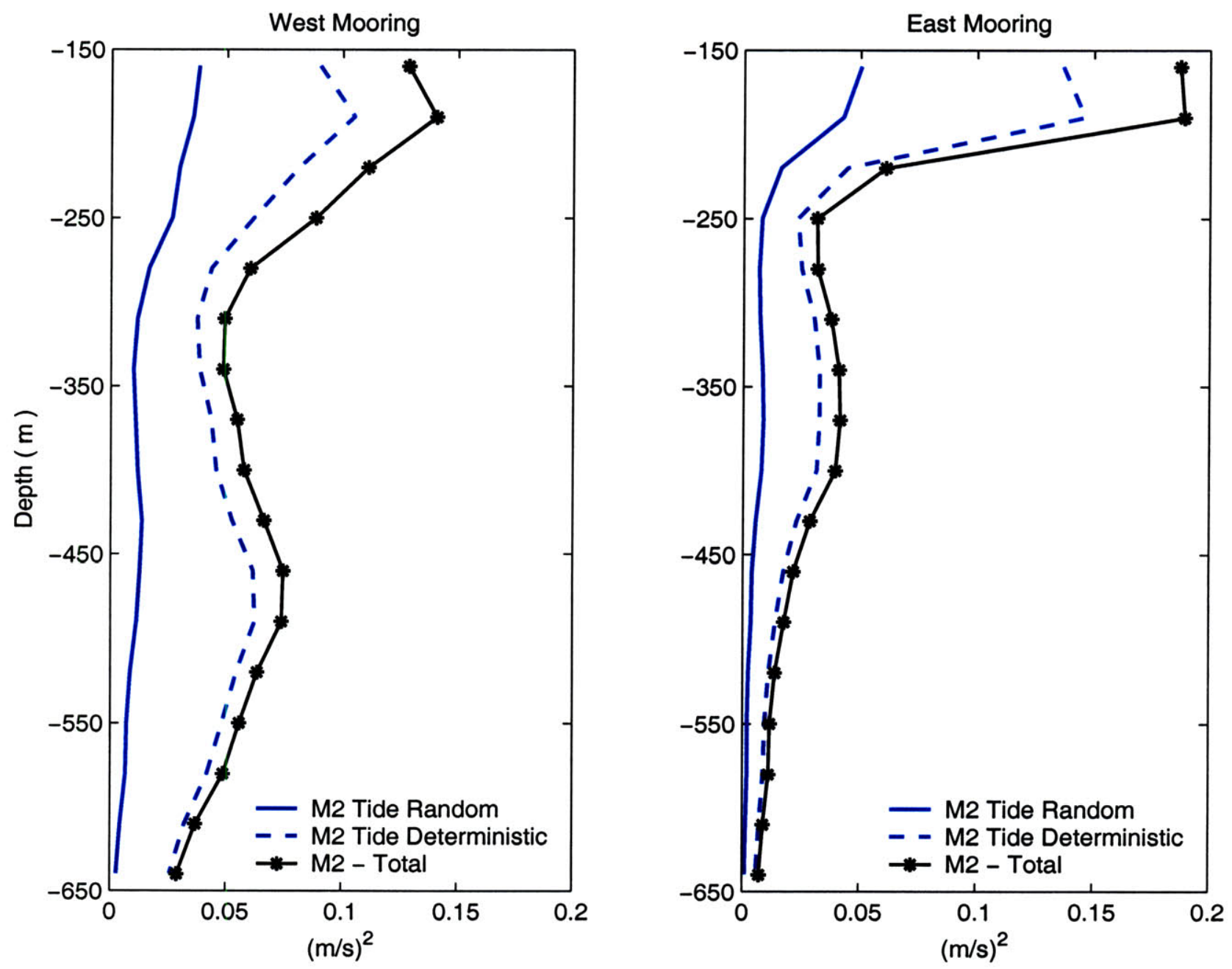

Figure 2-12: Sound speed variance due to the internal tide is subtracted from the total sound speed variance. M2 tide random and deterministic component are separated based on the extension of the power law continuum from high frequency. The variability is dominated by the deterministic tide. 


\subsubsection{Vertical structure of sound speed variance derived from XBT data}

Two types of XBT surveys were carried out to supply the measurement of the spatial variation of sound speed. The XBT profile includes not only the mean structure, but also the distortion of the mean profile by internal waves. In this sub-section, the spatial variation of temperature and sound speed will be presented. Sound speed fluctuation here is estimated approximately based on Chen and Millero's formula[10, 9], but with the constant salinity of 35 (PSU). Pressure is obtained using the depth of the XBT.

\section{High Resolution XBT Temperature Variability}

In addition to the moored observations, the high resolution calibrated XBT data can provide estimates of sound speed variability in the vertical direction. Figure 2-13 shows the observed sound speed variance for ten high resolution surveys. Fluctuations were computed by first averaging all casts in a given survey to get a mean sound speed profile and then this mean profile was subtracted to yield fluctuations. The separation of internal waves and other phenomena requires information in the time domain as well as in space. Note that in this case we cannot separate internal wave and mesoscale band contributions because we do not have a timeseries.

Thus these estimates must be interpreted in this light. That being said we clearly see more sound speed variance in these XBT observations than in the internal wave band of the moored observations. The depth structure of the sound speed variance is characterized by a mixed layer which is clearly seen in the upper $40-50 \mathrm{~m}$, followed by a two-peaked structure and smaller fluctuations at depths below $250 \mathrm{~m}$. The peak in sound speed variance at roughly 150-250 m depth is consistent with the rise seen in the moored observations up to the minimum moored instrument depth of $150 \mathrm{~m}$. In the mixed layer the sound speed variance is relatively small but non-zero presumably due to intrusive microstructure or spice effects[29]. The peak in sound speed variance directly under the mixed layer is due to the large gradient of temperature under the mixed layer and the undulations of the mixed layer lower interface (See Figure 2-5). 

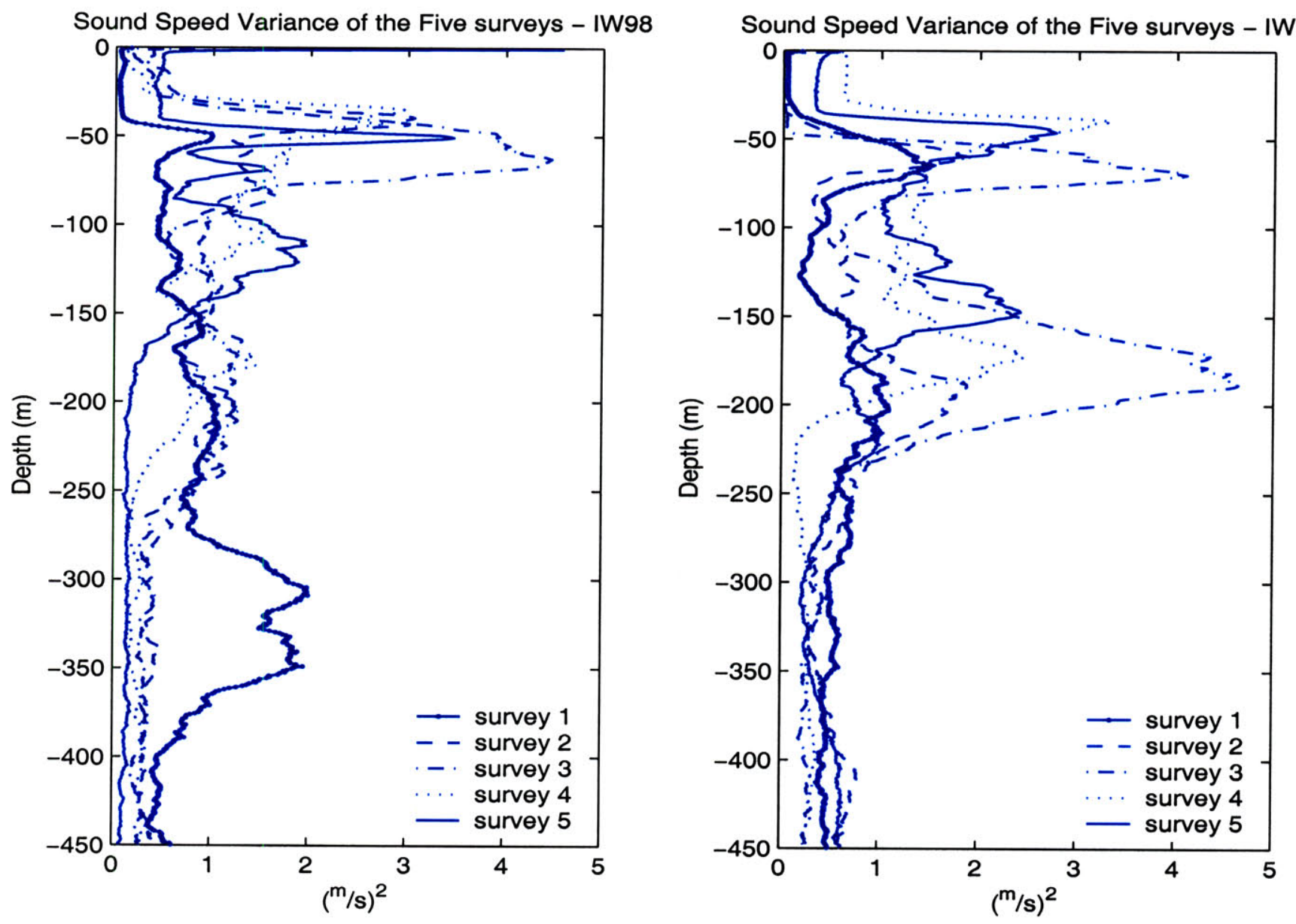

Figure 2-13: The spatial sound speed variance as function of depth based on the 5 high resolution XBT surveys in IW98 and IW99 cruise. The sound speed is derived from temperature, assumed constant salinity (35 PSU) and pressure (estimated from the falling time of XBT) by using the Chen and Millero's formula. 

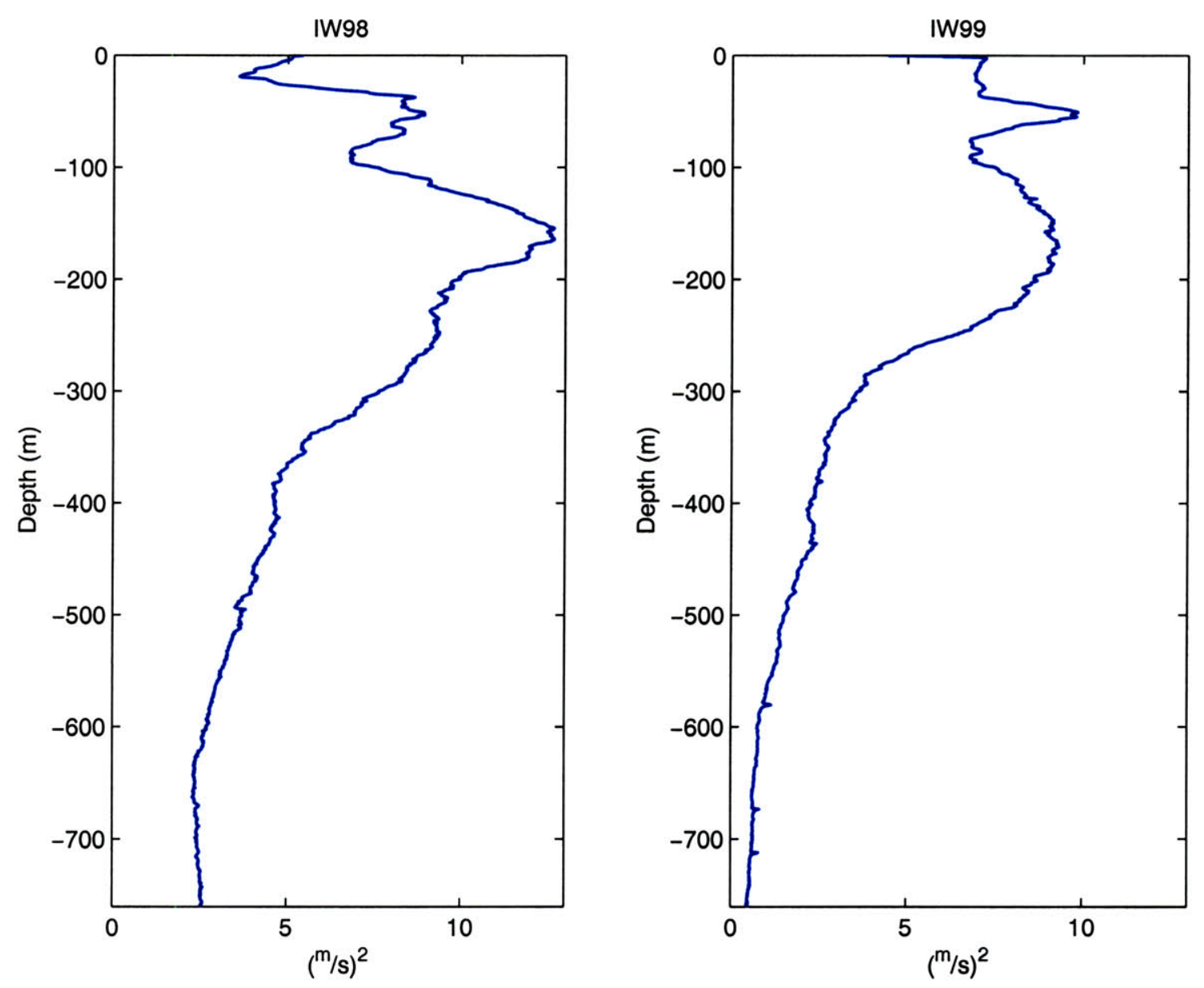

Figure 2-14: The spatial sound speed variance as function of depth based on the mesoscale XBT surveys in IW98 and IW99 cruise. The sound speed is derived from temperature, assumed constant salinity (35 PSU) and pressure (estimated from the falling time of XBT) by using the Chen and Millero's formula.

\section{Mesoscale XBT Surveys}

The two mesoscale XBT surveys conducted during the two deployment and recovery cruises provide "snap shot" views of the mesoscale field along the NPAL transmission path. Figure 2-4 shows these two sections, and subtracting out climatological values of temperature yields the lower panels for Figure 2-4. The mesoscale activity is clearly isolated near the island of Kauai and close to the California coast (California Current). Computing sound speed variance from these two sections shows the deep (750-m) variation in Figure 2-14. 


\subsection{Spectral Analysis of Sound Speed and Temperature}

The Fourier analysis method is used to calculate the frequency and the vertical wavenumber spectrum of sound speed of the mooring data, and the horizontal and vertical wavenumber spectrum of temperature from the XBT data. Spectrum analysis shows the sound speed and temperature fluctuation structure in the different frequency or wavenumber bands.

\subsubsection{Frequency Spectra of Sound Speed}

As in the last section, for the mooring data, the investigation of sound speed fluctuation is still focused on two different bands: sub-inertial and super-inertial bands, as shown in Figure 2-7.

\section{Frequency Spectra of Sound Speed in Sub-inertial Band}

After removing the mooring motion effect, the frequency spectrum of sound speed is computed by using moored time-series data at the 17 different depths from $160 \mathrm{~m}$ to $640 \mathrm{~m}$ at every $30 \mathrm{~m}$. A few examples of the frequency spectra of sound speed fluctuation in this sub-inertial band are shown in the two panels of Figure 2-15 for west and east mooring observation. The frequency spectra of sound speed in this sub-inertial band have very similar shape for all the frequencies: below $f$ the spectra drop sharply and then increase rapidly down to the lowest resolvable frequency, which is also clearly shown in Figure 2-7. In addition, sound speed spectra in the shallow depth have much larger variance than those in deeper depth. This is also shown in Figure 2-9 of sound speed variance. The vertical straight dash line shows the local inertial frequency, which is also the cutoff frequency of the digital low pass filter. The cutoff of the spectra in the high frequency is due to low pass filtering.

\section{Frequency Spectra of Sound Speed in Super-inertial Band}

The same spectrum estimation method is used to calculate the frequency spectra of sound speed in the super-inertial band at different depths. A few examples of such frequency spectra are shown in Figure 2-16 for the east mooring and west mooring, respectively. The vertical straight dot dash lines denote the local Coriolis frequency. The cutoff of the spectrum at low frequency is due to the high pass filtering. These measurements showed 

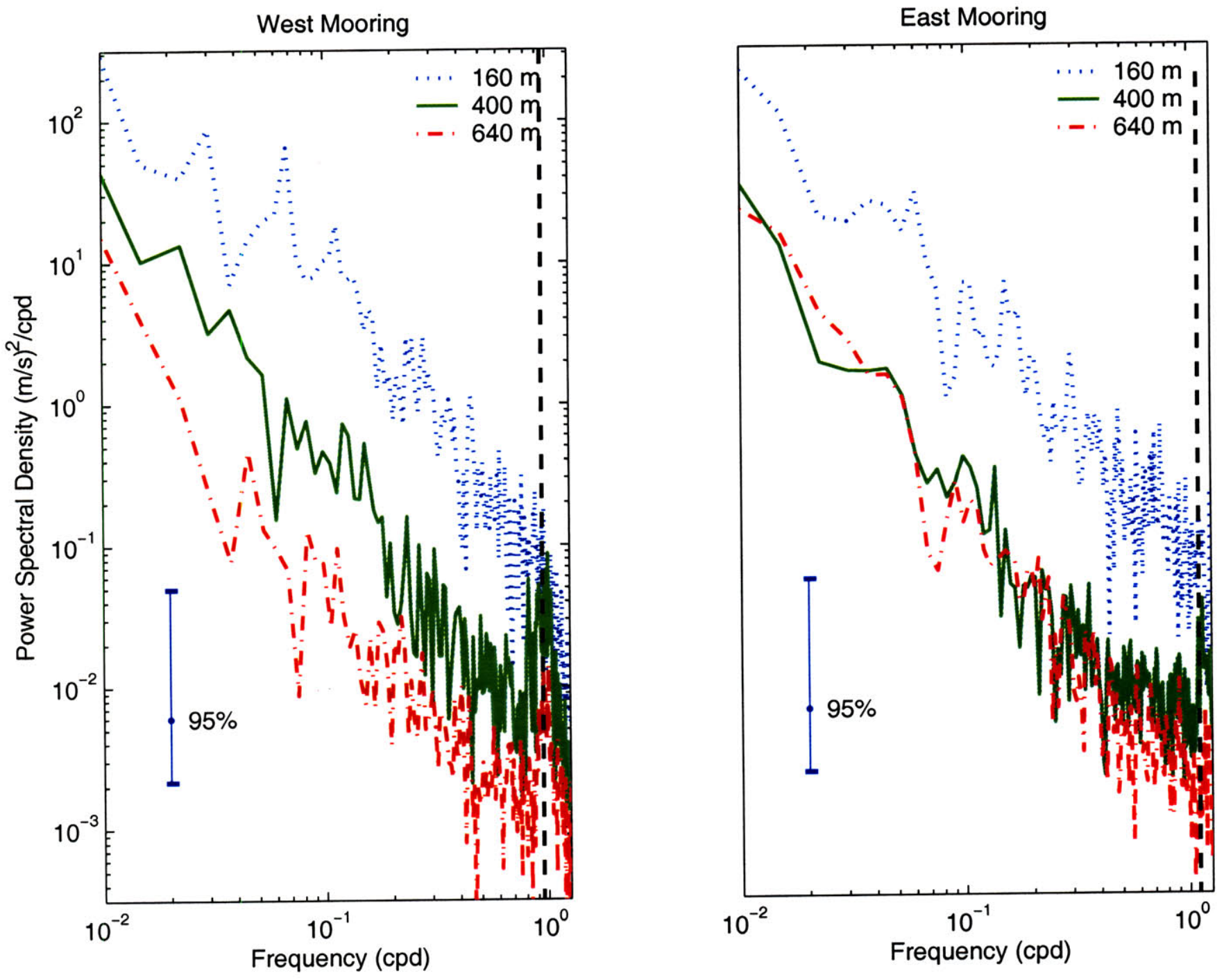

Figure 2-15: Frequency spectrum of the sound speed fluctuation in the sub-inertial band at the different depths for west and east mooring. The shallow depth has larger variance than the deeper depth. The vertical straight lines denotes the local Coriolis frequency, which is also the cutoff frequency of digital low pass filter. $95 \%$ confidence interval is drawed in the figures. The spectra cutoff in the high frequency is due to low pass filtering. 
that although they are different in detail, the frequency spectra of sound speed in the internal wave band have common basic feature. First, except the internal tide frequency and its harmonic frequency, they are almost continuous within the whole frequency range without pronounced maxima. Second, the spectra density level decreases with increasing

frequency with an $\sim \omega^{-2}$ dependence to about $20 \mathrm{CPD}(1 \mathrm{CPH})$ where the spectral slope begins to flatten, forming a "shoulder". At about 80-100 CPD, there is a sharp break followed by a rapid roll-off. Third, at the highest frequencies in the spectrum, a "shoulder" or buoyancy bump occurs as theoretically expected at the local buoyancy frequency.In addition, this buoyancy bump tends to move to the lower frequency region as the water depth goes deeper, because the buoyancy frequency gets smaller. And also the buoyancy bump tends to be more evident with increasing water depth, which may be caused by surface mixing or contamination in the shallow depth.

Next, the spectral estimation of observation is compared with the GM model (black dash lines). There is a sharp peak at the M2 tidal frequency which shows the most energetic region in this super-inertial band. The obvious difference between the data and the GM model is this coherent part of the semidiurnal tide and the bump at high frequency which is due to the internal wave cutoff at $\mathrm{N}(\mathrm{z})$. As we know, the internal wave can only exist in the frequency band between the local Coriolis frequency and buoyancy frequency. The GM model describes the internal wave very well in this band, especially in the deep water depth, while in the shallow water depth such as $160 \mathrm{~m}$, the deviation from the GM model is observable in the high frequency region. But in the east mooring data, the spring spectrum shows deviation from other seasons at the depth of 640 meter.

Seasonal Variability of the Sound Speed Frequency Spectra in Super-inertial Band

To investigate the seasonal variation of the frequency spectrum of sound speed, the frequency spectrum is calculated in different seasons at different depths. The depths at $160 \mathrm{~m}, 400 \mathrm{~m}$ and $640 \mathrm{~m}$ are selected to show the seasonal variation at different depths in Figure 2-17. The four-season frequency spectra at different depths of the west mooring data are shown in upper panels. The lower panels display the eastern mooring data. The vertical straight black dash line shows the local inertial frequency. In general, the season effects are much more visible in the shallow water than in deep water. But at some great depths, the winter 

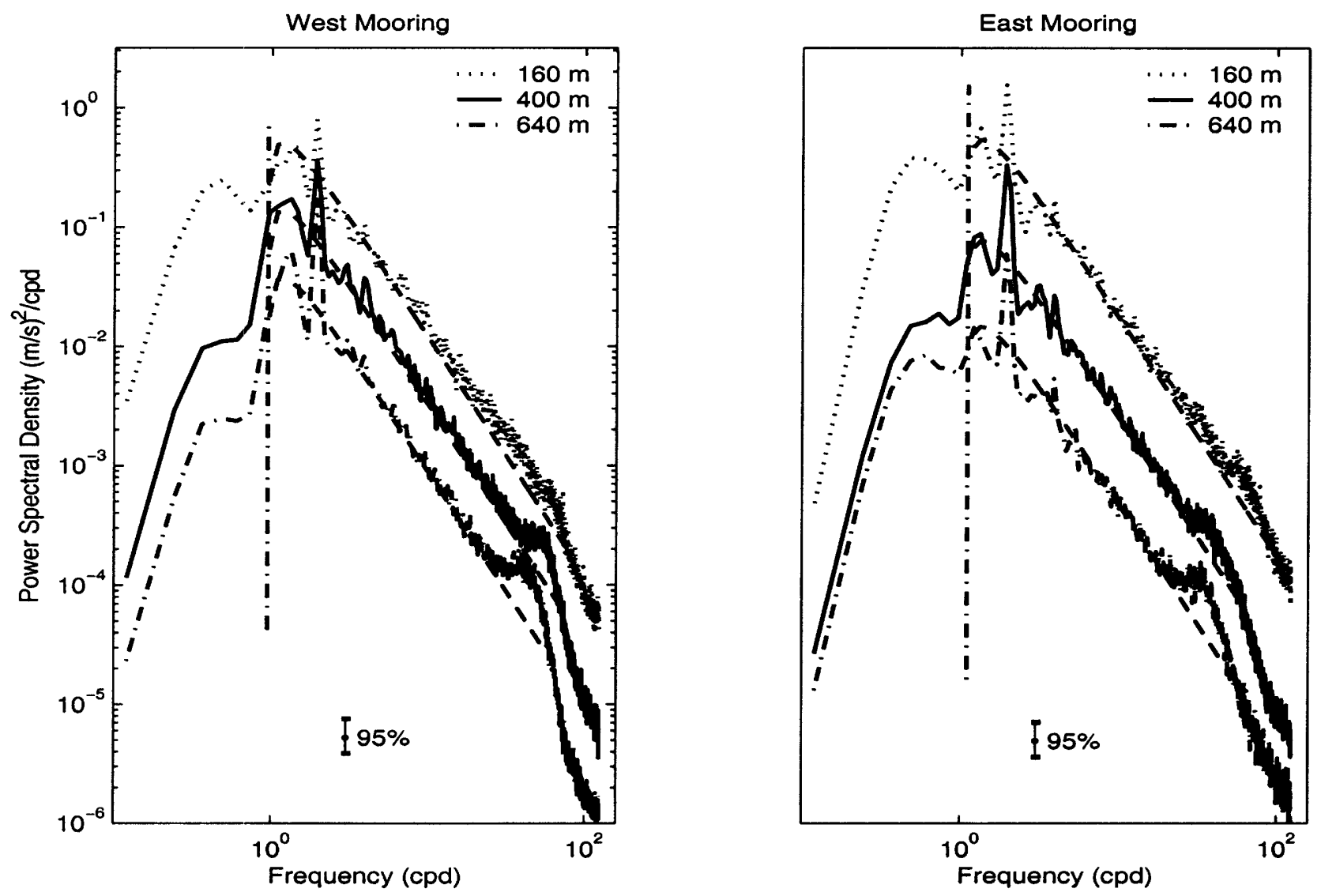

Figure 2-16: Comparison between GM model and the frequency spectrum of the sound speed fluctuation in super-inertial band at different depths. The semidiurnal tide and buoyancy bump are evident in the spectrum display. The GM model with parameter $N_{0}=3 \mathrm{cph}$ of a reference buoyancy frequency, and $\zeta_{0}=7.3 \mathrm{~m}$ of a reference rms internal wave displacement are plotted with dash lines. GM model is normalized with the variance of sound speed to be shown with data together. The vertical straight dot dash lines denote the local Coriolis frequency. The cutoff of the spectrum at low frequency is due to the high pass filtering. $95 \%$ confidence intervals are narrower to compare with the ones of sub-inertial frequency spectrum, which is due to more degrees of freedom are obtained to use shorter FFT length to compute the super-inertial data. 
effect is still visible, such as the $640 \mathrm{~m}$ depth of the east mooring.

\subsubsection{Vertical Wavenumber Spectra of Potential Temperature}

Since the salinity data is only collected at six layers, to avoid errors introduced by too much interpolation, only the potential temperature data is used to investigate the vertical structure of sound speed fluctuation in the upper ocean. As we know, the spectra analysis in the vertical direction presents a unique problem in that the random processes which describe the fluctuations are not statistically stationary due to the nonhomogeneity in the vertical direction. To reduce this effect due to depth variations in buoyancy frequency, one standard technique of WKB-stretching is employed, which will render the fluctuation process statistically stationary to the first order approximation [Bell, 1974;Pinkel,1984][4, 74]. The potential temperature is derived from the moored temperature, salinity and pressure data. By and large, a set of 16-layer data over the whole mooring deployment period was obtained. First, the time domain frequency Fourier analysis was applied to the potential temperature data. Then, for each frequency band between the local inertial frequency and local buoyancy frequency, the depth series were WKB-weighted and their first order differences in depth were taken. A Fourier transform with hanning window was employed to get the vertical spectrum of potential temperature. Seasonal variations of vertical spectrum of potential temperature are displayed in Figure 2-18.

In Figure 2-18, the black line with the dot markers is GM vertical wavenumber spectrum. Basically, the vertical spectrum of potential temperature $(\theta)$ shows GM-like shape in data from both moorings. A very sharp cutoff is shown in the scale of $150 \mathrm{~m}$ in both two mooring panels which show the internal wave limited band in the vertical direction.

To observe the frequency dependence of the vertical spectrum of potential temperature, the autumn season was chosen to show the vertical spectrum in the frequency band from 1 CPD up to $53 \mathrm{CPD}$ in Figure 2-19

Although the vertical spectrum is GM-like shape in different seasons, the different structure of vertical spectrum in different frequency bands is significant, which indicates the limitation of GM model's factorization in terms of frequency and wavenumber. 

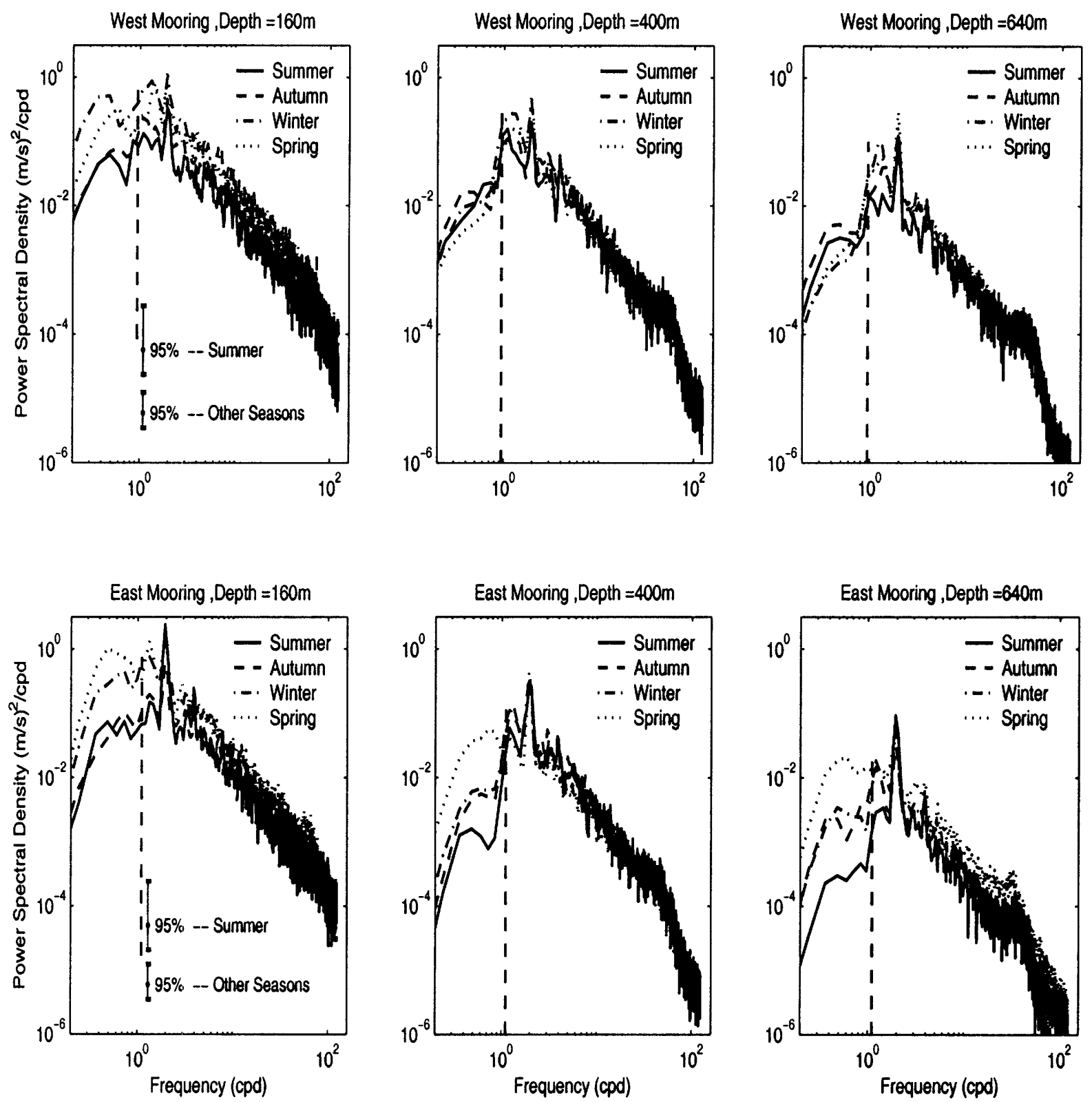

Figure 2-17: The seasonal variation of sound speed spectrum at depths of $160 \mathrm{~m}, 400 \mathrm{~m}$, $640 \mathrm{~m}$ of western and eastern moorings. The vertical straight dash lines denote the local Coriolis frequency. $95 \%$ confidence intervals are plotted for the summer and other seasons. Because the data length of the summer season is shorter than the other seasons, the summer confidence interval is wider than those of the other seasons. 

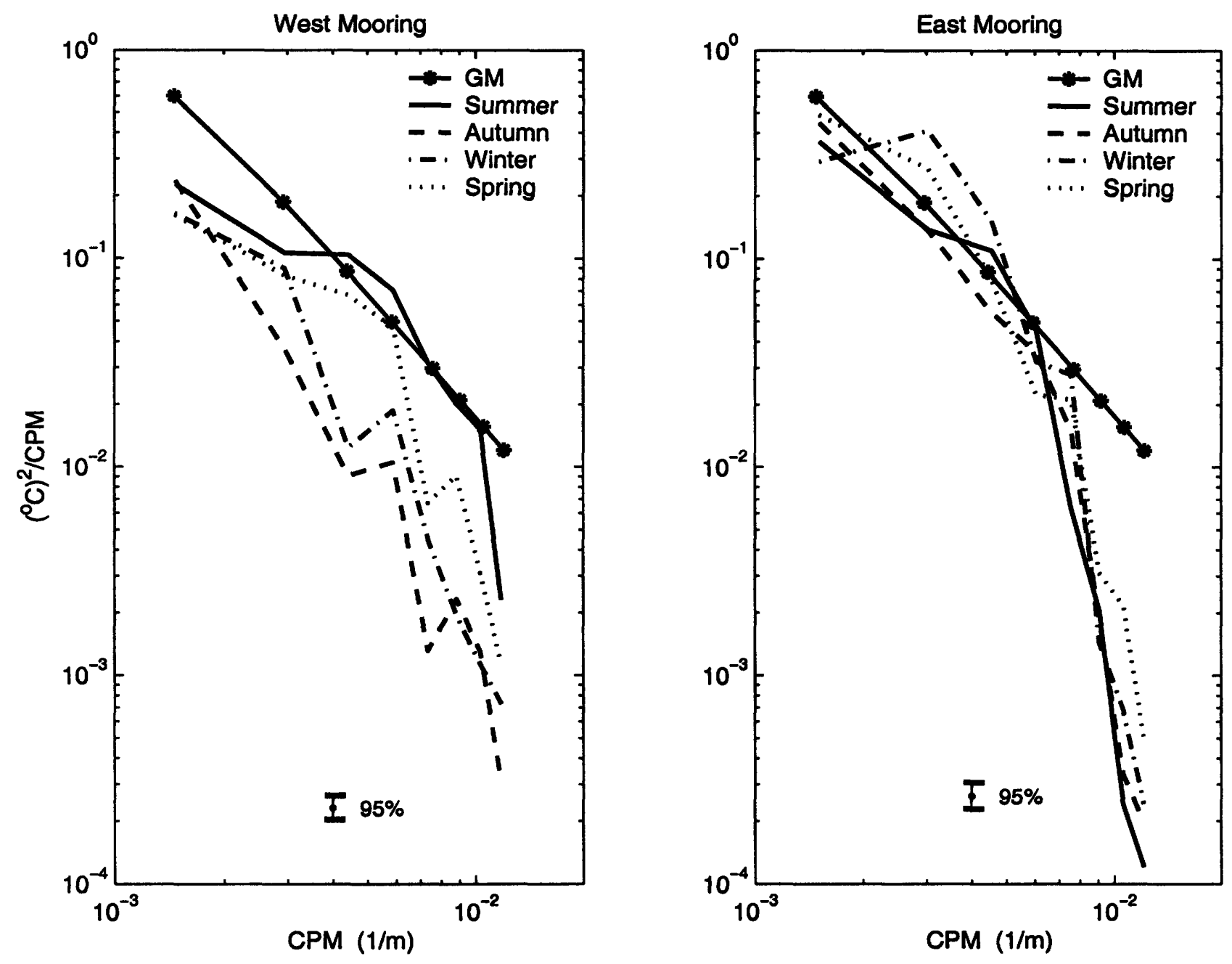

Figure 2-18: Seasonal variation of the vertical wavenumber spectrum of potential temperature for western and eastern moorings. The vertical spectrum is only estimated in the frequency band between the local Coriolis frequency and local buoyancy frequency. The potential temperature is derived from the moored temperature, salinity and pressure data. WKB-stretching method is applied to reduce the non-stationary fluctuation due to the nonhomogeneous in the vertical direction. Buoyancy data used here are derived from the CTD data during the cruise. The dot-marker lines shows the GM model to compare with the observation. 

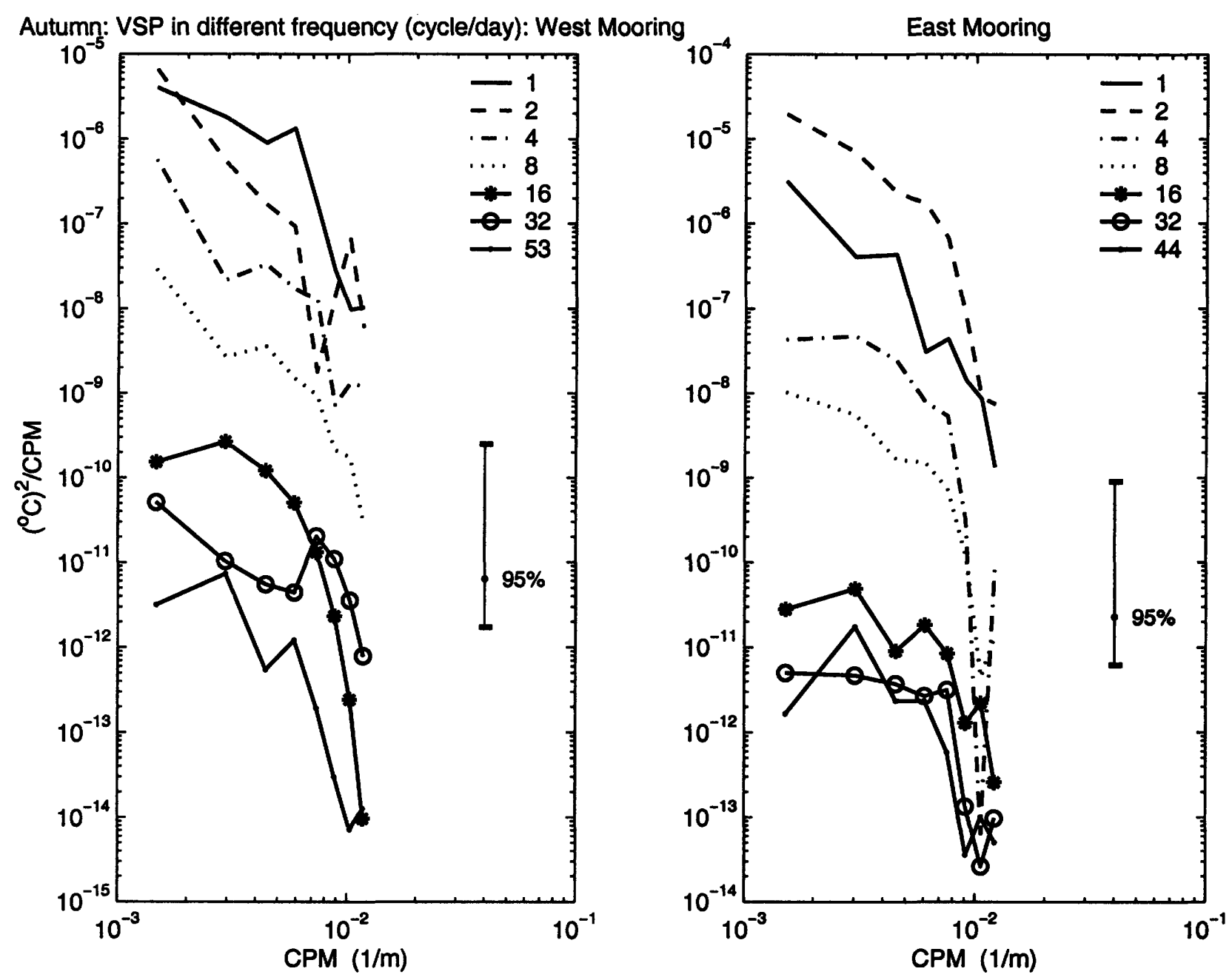

Figure 2-19: Vertical wavenumber spectrum of potential temperature in different frequency bands for west and east moorings. The dependence on the different frequency bands of the vertical spectrum shows the GM model limitation. 


\subsubsection{Horizontal and Vertical Wavenumber Spectra of Temperature}

High resolution deployment of XBT during the cruises provides us a chance to observe the horizontal and vertical wavenumber spectra of temperature. The sound speed is mainly a function of the temperature. Accordingly the figure of the temperature shows the sound speed structure approximatively. As shown in Figures 2-5 and 2-13, the mixing layer is apparently shown in the upper layer with about 50 meter depth of the ocean. All the horizontal and vertical wavenumber spectra are only estimated by using the data below the mixing layer. All the linear trends are removed from the data before the Fourier analysis is applied. Figure 2-20 shows the vertical spectrum of temperature which is the average of the all five high resolution surveys' vertical spectra. The buoyancy frequency, which is used in the WKB stretch approach, is derived from the CTD measurement as shown in the Figure 2-6. The dash line is the GM vertical spectrum for comparison. The temperature vertical wavenumber spectrum shows a cutoff at vertical scale of 1 to 10 meters. The vertical spectrum of temperature gradient in Figure 2-21 shows this cutoff very clearly. This cutoff indicates the temperature fluctuation is associated with a minimum scale just as the internal wave fluctuation[49].

In Figure 2-22, the comparison between the XBT data and the GM model is also made. The horizontal wavenumber spectrum of XBT is the average of the five surveys. Both the horizontal and vertical wavenumber spectra show GM-like shape.

\subsection{Summary}

In order to interpret the observed acoustic variability of sound transmission in the North Pacific ocean, a significant oceanographic measurement was conducted in the field year August 1998 to June 1999 of NPAL experiment. We have shown some results from a study of the in situ environmental data. Of particular interest are the space time scales of the upper ocean sound speed fluctuation caused by mesoscale eddies, internal tides, and internal waves and other fine scale processes. We have separated sound speed variability into two frequency bands: greater than, or less than the Coriolis frequency. But we cannot easily separate internal wave effects from spice due to intrusive effects. The time and space sound speed variance, frequency and wavenumber spectrum of sound speed in different frequency bands and seasonal variation from extensive collected data are presented, and also with the 

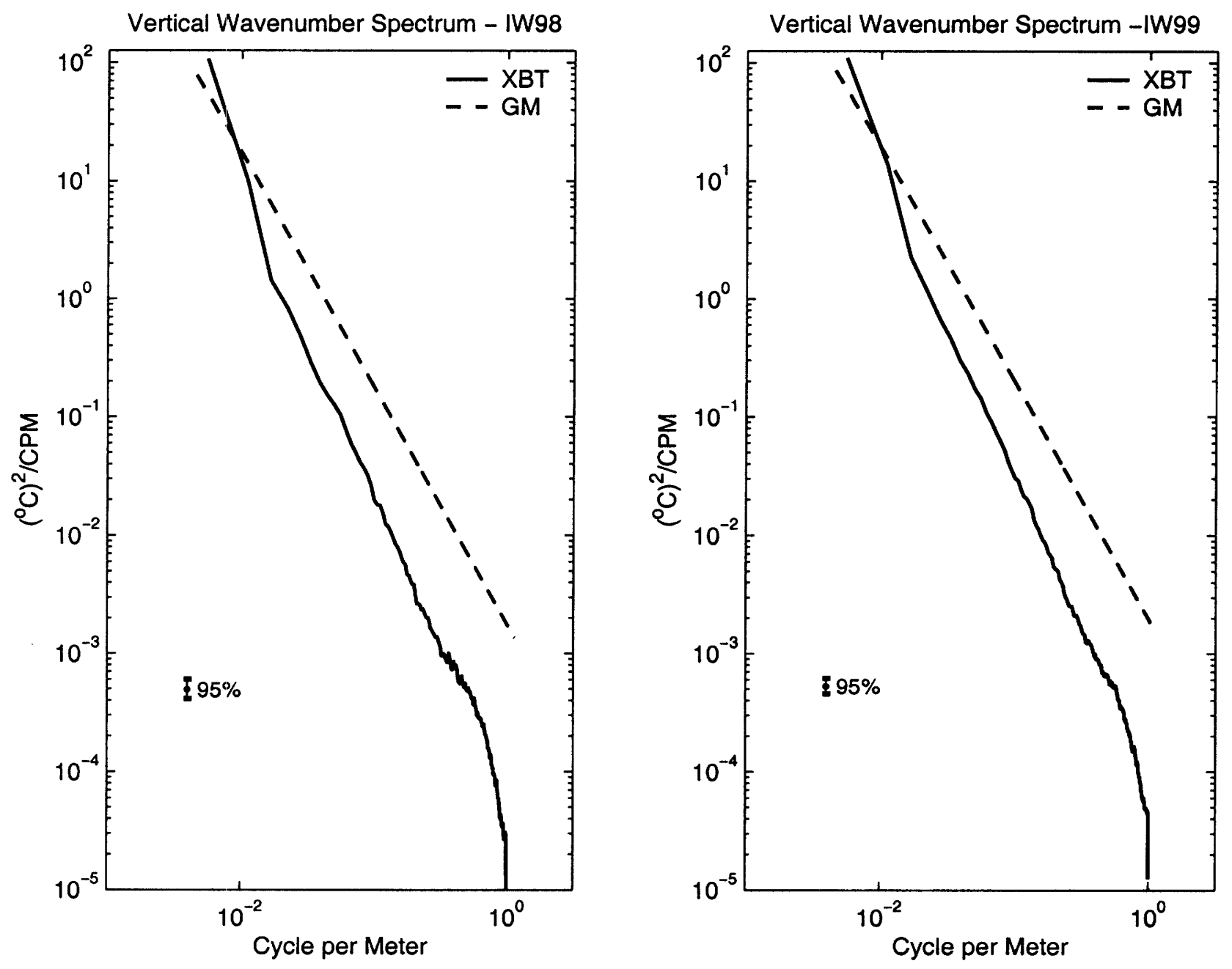

Figure 2-20: Vertical wavenumber spectrum of temperature from five high resolution XBT surveys of IW98 and IW99 cruises. Only the data below the mixing layer is used to to compute the spectrum. The dash line is GM model for comparison. 

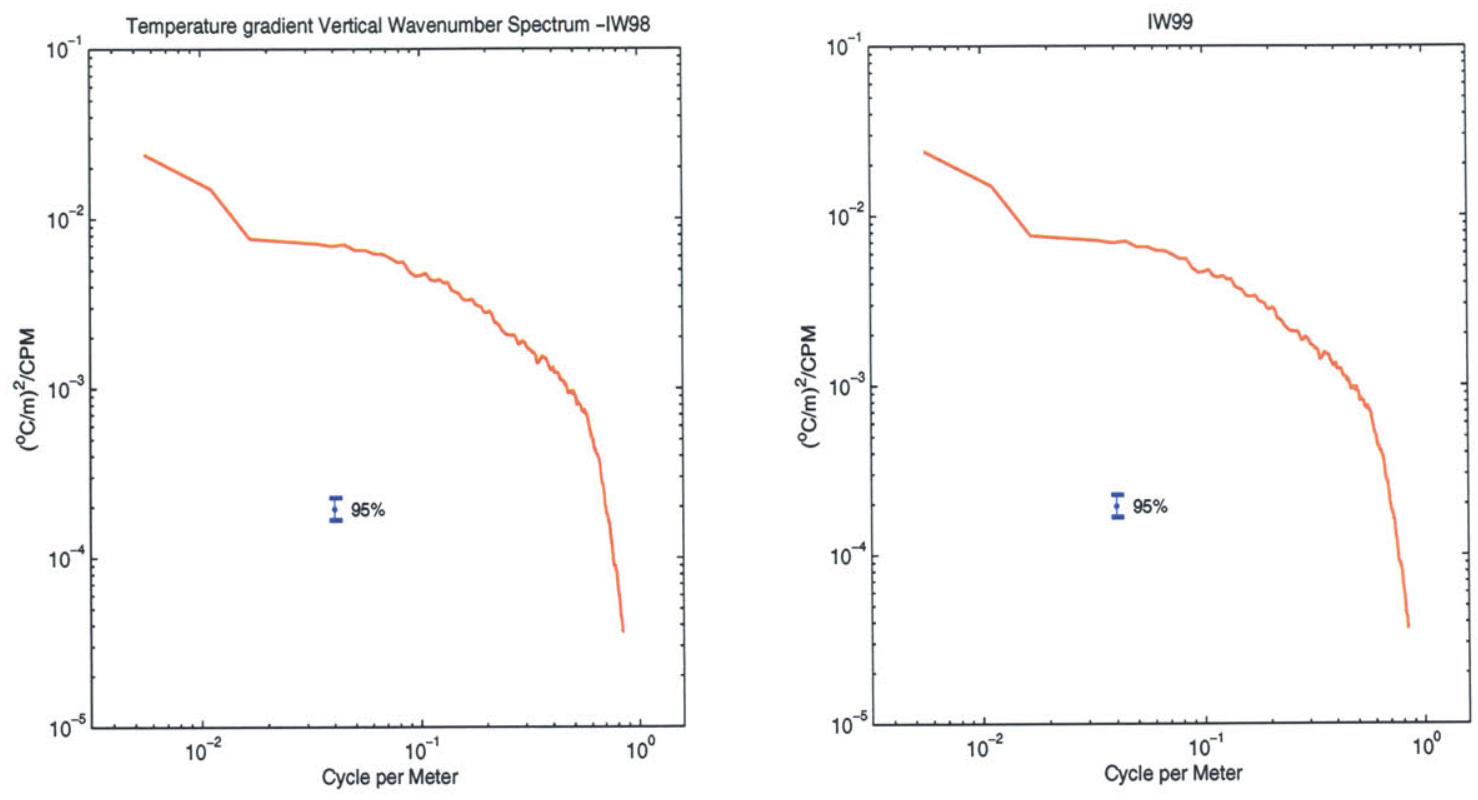

Figure 2-21: Vertical wavenumber spectrum of temperature gradient from five high resolution XBT surveys of IW98 and IW99 cruises. Only the data below the mixing layer is used to to compute the spectrum.
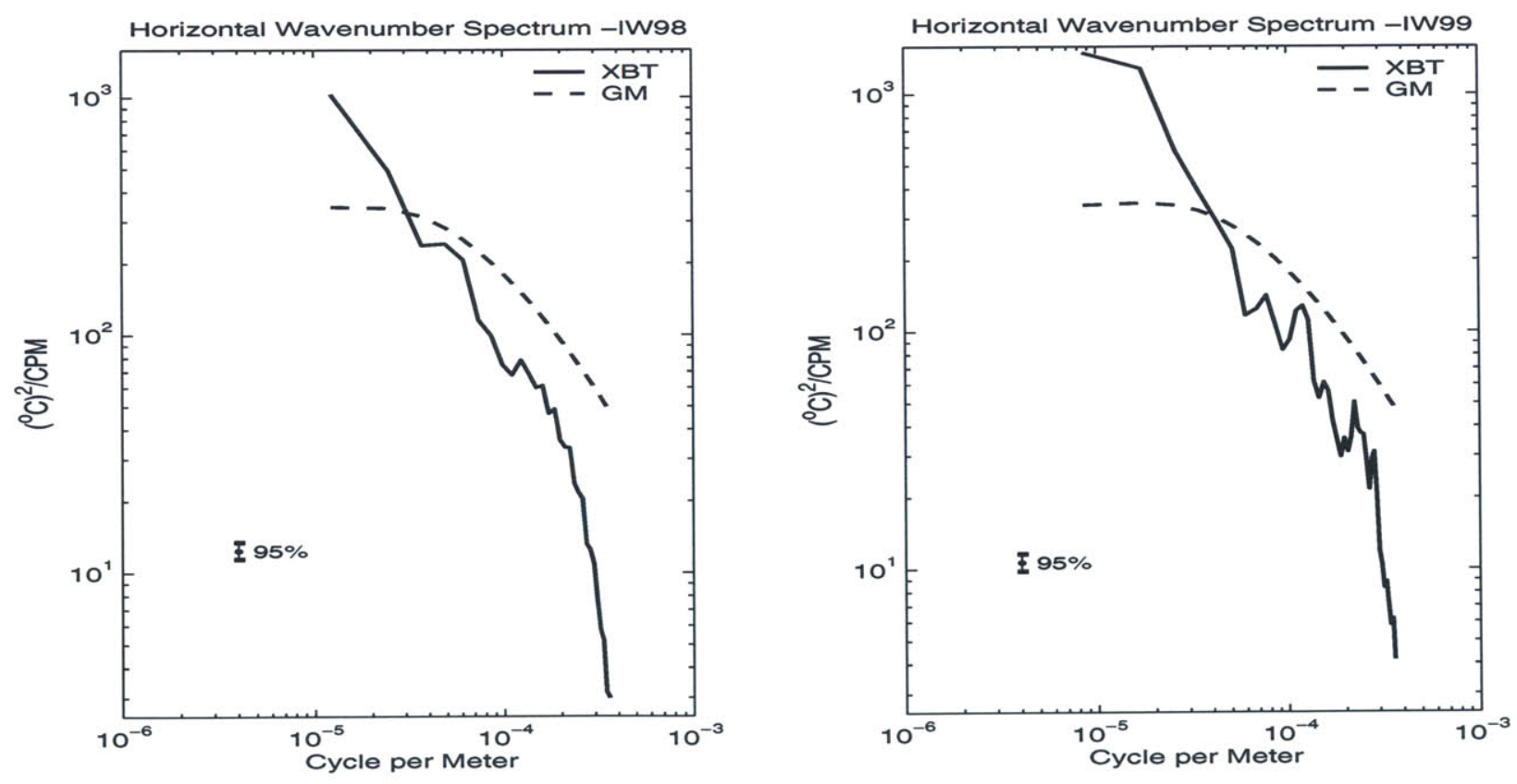

Figure 2-22: Horizontal wavenumber spectrum of temperature from five high resolution XBT surveys for IW98 and IW99 cruise. Only the data below the mixing layer is used to to compute the spectrum. The dash line is GM model for comparison 
wavenumber spectrum of temperature to compensate for understanding spacial variation. The result shows the mesoscale fluctuations contains more sound-speed variance than the internal wave band fluctuation. The maximum mesoscale fluctuation is of order $4 \mathrm{~m} / \mathrm{s} \mathrm{rms}$. The internal wave band has a maximum fluctuations of $1-1.5 \mathrm{~m} / \mathrm{s} \mathrm{rms}$ with significantly smaller values at depth. The frequency spectra in the sub-inertial band increase as $\omega^{-2}$ as frequency decreases; Frequency spectra in the IW band are very GM-like. The spectral levels do vary with season, with higher energy in the winter and spring seasons. Seasonal sensitivity diminishes with depth. In general, the comparison with the theory result shows the GM internal wave model is a well set-up model under certain conditions (such as in this region of North Pacific).

Internal tides provide a small contribution to the overall variance in the IW band. The variability in the internal tides band is dominated by the deterministic tide of the $M_{2}$ peak energy, but compared to the total internal wave band energy the $M_{2}$ is quite small, which is less than $15 \%$ contribution of total internal wave field. Therefore, the GM model may underestimate the sound speed variance, but only by this small amount(Fig. 2-12).

The comparison of the sound speed variance from the mooring stations and the canonical model indicates that the ratio of sound speed variance and the internal wave variance is not universal. For example, in the east mooring, the observed sound speed variance is only one-half that of GM spectrum (See Fig.2-11).

For XBT data, sound speed variance in the upper ocean shows two-peaked structure. Vertical wavenumber spectrum of temperature is GM-like and $k^{-2.5}$ dependence. Vertical wavenumber spectrum of the temperature gradient show a cut-off at $1-5 \mathrm{~m}$. Horizontal wavenumber spectrum scales like $k_{x}^{-1.5}$.

In general, we have shown some results from the study of in situ environmental data. These observations provide significant quantification of space-time scales of ocean sound speed variability in both the internal wave and mesoscale frequency bands. All those results impose hard bounds on the strength of sound speed fluctuations one might expect in the region of the North Pacific for both internal-wave band fluctuations and mesoscale band fluctuations. 


\section{Chapter 3}

\section{Observations and Modeling of}

\section{Low-frequency Sound Wave}

\section{Propagation in the Random Deep}

\section{Ocean}

\subsection{Introduction}

Due to the emphasis on ocean acoustic remote sensing and tomography in the last two decades, observational efforts to study acoustic scattering of low frequency sound (of order $30-300 \mathrm{~Hz}$ ) in the ocean have focused on effects at very long ranges, between 1000 and 15,000 km (SLICE89, AET, ATOC, Heard Island, etc). Several important discoveries have been made, of which three are mentioned here. First, it has been shown that low frequency, broadband wavefronts are partitioned into two regimes: the part of the wavefront that is composed of high grazing angle rays or higher order modes(often termed the ray-like region of the wavefront) showing well separated(in time and space) quasi-planar fronts with small fluctuations in intensity and travel time, while the part of the wavefront that is composed of low grazing angle rays or low order modes(often termed the wavefront finale) shows a complicated multipath interference pattern with large fluctuations similar to Gaussian random noise[22, 23]. Second, for both regimes it has been shown that the edges of the shadow zones of the wavefront are significantly extended in depth, and in 
time[19, 20][26]This extension of the shadow zone shows that the effect of scattering in long range low frequency ocean acoustic propagation is to introduce a significant bias into the wavefront intensity pattern; that is to say, the acoustic fluctuations cannot be considered a zero mean effect superimposed upon an otherwise deterministic wavefront pattern. Finally, in spite of the large fluctuations in the wavefront finale the time stability of the phase is surprisingly large and close to that of the wavefront region[22, 79, 30]. Observed coherence times are between 5 and 15 minutes.

It has been shown that the strongest acoustic scattering occurs near a ray upper turning point (UTP) [58] so long range acoustic propagation of order $1000-\mathrm{km}$, involves of order 10 to 20 scattering events. Previous work on single UTP propagation has been entirely at frequencies of $1000 \mathrm{~Hz}$ or more (Worcester (SD),Ewart (MATE), Flatté (AFAR)) [86, 35, 42], and it is not directly related to the low frequency cases. So, we present an analysis of acoustic transmission data obtained in the Northeast Pacific Ocean as part of the ATOC project's Acoustic Engineering Test (AET) $[22,83]$. Here 75- $\mathrm{Hz}$ signals with an bandwidth of $30 \mathrm{~Hz}(3 \mathrm{~dB})$ were transmitted to a range of $87 \mathrm{~km}$, and were received on a 700-m long 20 element hydrophone array. The arrival pattern of these transmissions consists of two time-resolved and identifiable wavefronts; one with an initially downwards ray angle and two lower turning points (LTP) and one UTP, to be referred to as ID -3, and the other arrival with an initially upwards ray angle and two LTP's and two UTP's, to be referred to as ID +4. Because acoustic scattering is most pronounced near the UTP these two arrivals provide a view into the fundamental scattering physics at the first two UTP's.

The scattering physics model we consider is due to Munk and Zachariasen (MZ) (1976)[71] who modified Rytov's weak fluctuation theory of optical propagation through a turbulent atmosphere, to the considerably more complex problem of ocean acoustic propagation through internal waves. The basic physics of the $\mathrm{MZ}$ model that is to be tested is that there is weak, single forward scattering, and that there is a resonance condition between the sloping ray path and the internal waves whose crests are aligned with the sloping ray. The resonance condition causes an important selectivity in acoustic/internal wave interactions such that rays with steep grazing angles can be too steep to interact with the low frequency part of the internal wave field. To test some of the assumptions of the MZ theory, and to help interpret the observational results we also perform parabolic equation (PE) Monte Carlo simulations for both broadband and narrow band cases. 
Briefly, our results show that observed intensity fluctuations are very weak, and thus consistent with the application of a perturbative theory like the MZ theory. Scintillation index $(\mathrm{SI})$, and variance of $\log$-intensity $\left(\sigma_{\ln I}^{2}\right)$ over the 6 days of the observations give $S I=$ 0.04 and $\sigma_{l n I}=0.8 \mathrm{~dB}$ for ID -3 (one UTP), and $S I=0.4$ and $\sigma_{l n I}=3 \mathrm{~dB}$ for ID +4 (two UTP's). Most importantly however are the timescales of the observed intensity variability; that is the observed frequency spectra of intensity for ID's -3 and +4 . The spectra show that the steeper grazing angle arrival -3 shows much less low frequency variability than the lesser grazing angle arrival +4 , which is order of magnitude consistent with the $\mathrm{MZ}$ physics of the ray/internal wave resonance. We believe that this the first observational evidence of this resonance. The space scales of intensity variability are also of interest and we find that the vertical wavenumber spectra of intensity also show order of magnitude agreement with the MZ theory.

Regarding results for phase we are only able to examine phase over very short timescales between 1 minute and 40 minutes, because of gaps in the transmission schedule where phase could not be tracked. This problem clearly does not exist for the intensity observations. Thus phase variances are very small of order $0.6 \mathrm{rad} \mathrm{rms}$, and roughly the same for the two ID's. Comparisons with the MZ theory for the frequency spectrum of phase are poor because the observation timescales were marginally in the band where internal waves exist. Regarding vertical wavenumber spectra of phase, however, the agreement between the MZ theory and the observations is very good.

The outline of this chapter is as follows. In section 2 we describe the observations and the processing needed to obtain the phase and intensity data used in our analysis. In section 3 we present the various moments of phase and intensity, as well as the frequency and wavenumber spectra. The $\mathrm{MZ}$ theory and its comparisons to direct numerical simulation are presented in section 4 and section 5 compares observations and theory. Section 6 summarizes the results.

\subsection{AET Experiment and Reduction of the data to Phase and Amplitude}

The Acoustic Engineering Test (AET) for the ATOC program was conducted in the Eastern North Pacific Ocean from November 17,1994 through November 23, 1994[83]. The acoustic 
source was suspended from the research platform FLIP which was moored roughly 400 miles south-southwest of San Diego with coordinates $31^{\circ} 2.050^{\prime} \mathrm{N}, 123^{\circ} 35.420^{\prime} \mathrm{W}$, in water more than 4000m depth (Fig 3-1). From the sound channel axis(650-m depth) the source transmitted phase modulated signals ${ }^{1}$ with a center frequency of $75 \mathrm{~Hz}$ to two $700-\mathrm{m}$ long 20-element autonomous vertical line array(AVLA) receivers; one located 3250-km distant off the island of Hawaii and the other (the topic of this chapter) located 87-km distant and to the South of FLIP (Fig 3-1). The receiving array at $87 \mathrm{~km}$ range was located at $30^{\circ} 14.798$ ' $\mathrm{N}$ latitude, $123^{\circ} 36.4906^{\prime} \mathrm{W}$ longitude and spanned the depth range 900 to $1600-\mathrm{m}$.

During the AET experiment, a typical transmission sequence consisted of 40 consecutive broadcasts of a 27.28 second long phase coded signal ${ }^{2}$, which after pulse compression processing yielded a 40-point time series of the evolution of the time front. Figure 3-2 shows two examples of the timefront obtained by pulse compression of two different 27.28 second transmissions. Thus, every 27.28 seconds we obtain a record of pressure as a function of depth $z$, and as a function of travel time $T$, so we write the observed acoustic pressure as $p(z, T, t)$ where geophysical time coordinate $t$ has its smallest increment in units of 27.28 seconds. After the 40 consecutive transmissions, there would be $\mathbf{2}$ or 4 hour quiet period after which another set of 40 transmissions would be made. Over the 6 days of transmissions, the only exception to this pattern was that on alternate days, two 80 transmission sequences were done, followed by a 4 hour quiet period[83]. Thus in total there were $3 \times 12+3 \times 8=60$ short sequences with 40 transmissions each, and 6 long sequences with 80 transmissions each. Because of various technical problems, however, we found that only 24 of the 60 short sequences were usable, and only 3 of the 6 long sequences were usable. Data were deemed unusable if there were any hydrophone channels that were corrupted or missing over the entire 40 or 80 transmission sequence.

\subsubsection{Ray Identification}

Examination of Fig.3-2 shows two high intensity wavefronts sweeping by the vertical receiver array. At later travel times weaker bottom interacting arrivals are seen, but these will not be considered in the present analysis. Using the sound speed profile obtained from CTD casts during the deployment and recovery of the receiving arrays, a ray trace calculation was

\footnotetext{
${ }^{1}$ The source level was $260 \mathrm{~W}(195 \mathrm{~dB}$ re $1 \mu \mathrm{Pa}$ at $1 \mathrm{~m})$.

${ }^{2}$ The phase modulation was encoded using a linear maximal-length shift register sequence containing 1023 digits. Each digit was then $26.667 \mathrm{~ms}$ in duration, and each sequence period was $27.2800 \mathrm{~s}$ long.
} 

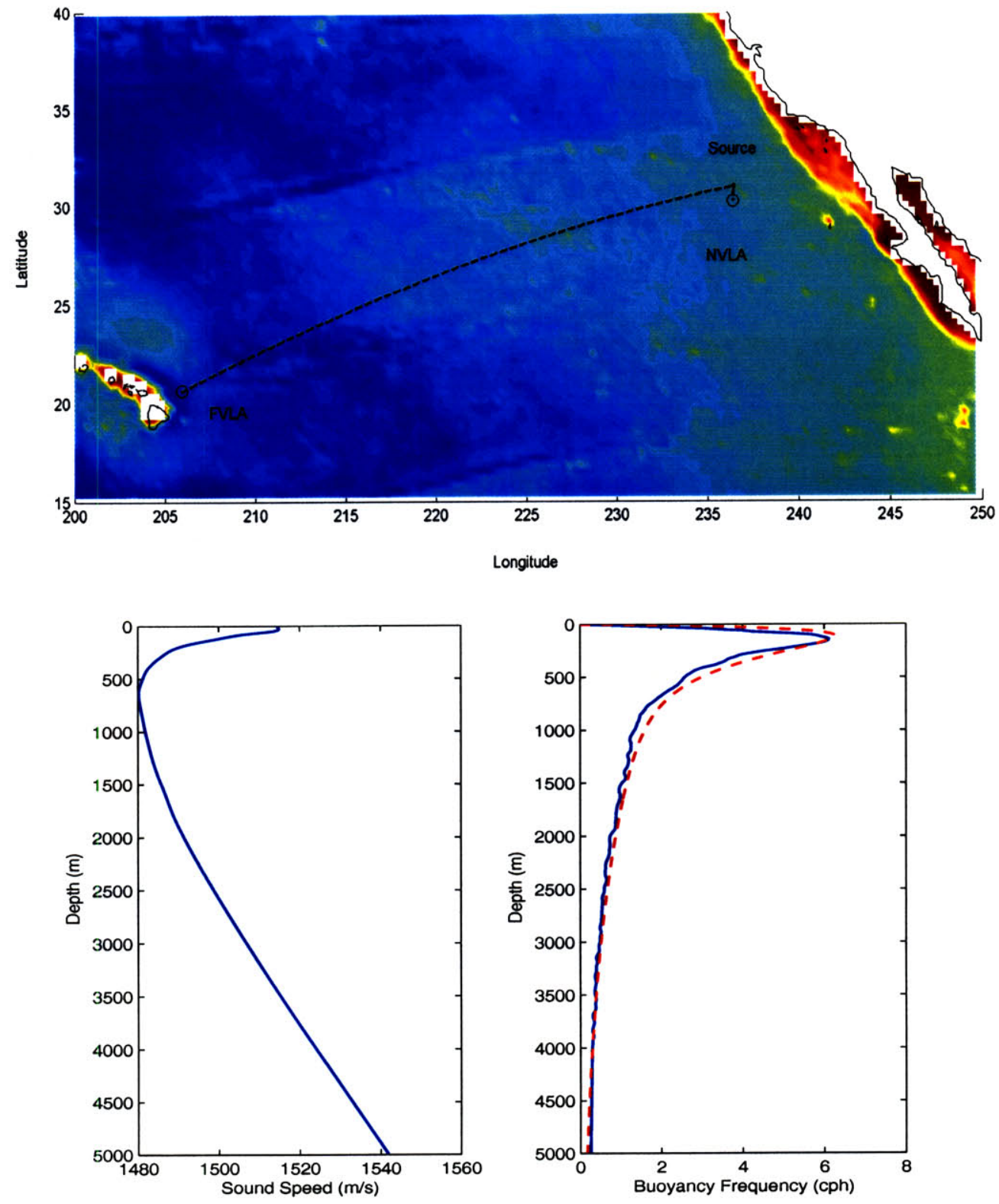

Figure 3-1: Map showing the location of the AET experiment in the North Pacific. The research platform FLIP occupied the source location and two 700-m long vertical receiving arrays were located at the NVLA and FVLA positions. Also shown are sound speed and buoyancy frequency profiles derived from CTD casts at the deployment and recovery of NVLA. A canonical fit (red) to the measured $N(z)$ profile (blue) is also shown. The mixed layer depth is between 25 and 35 meters. 

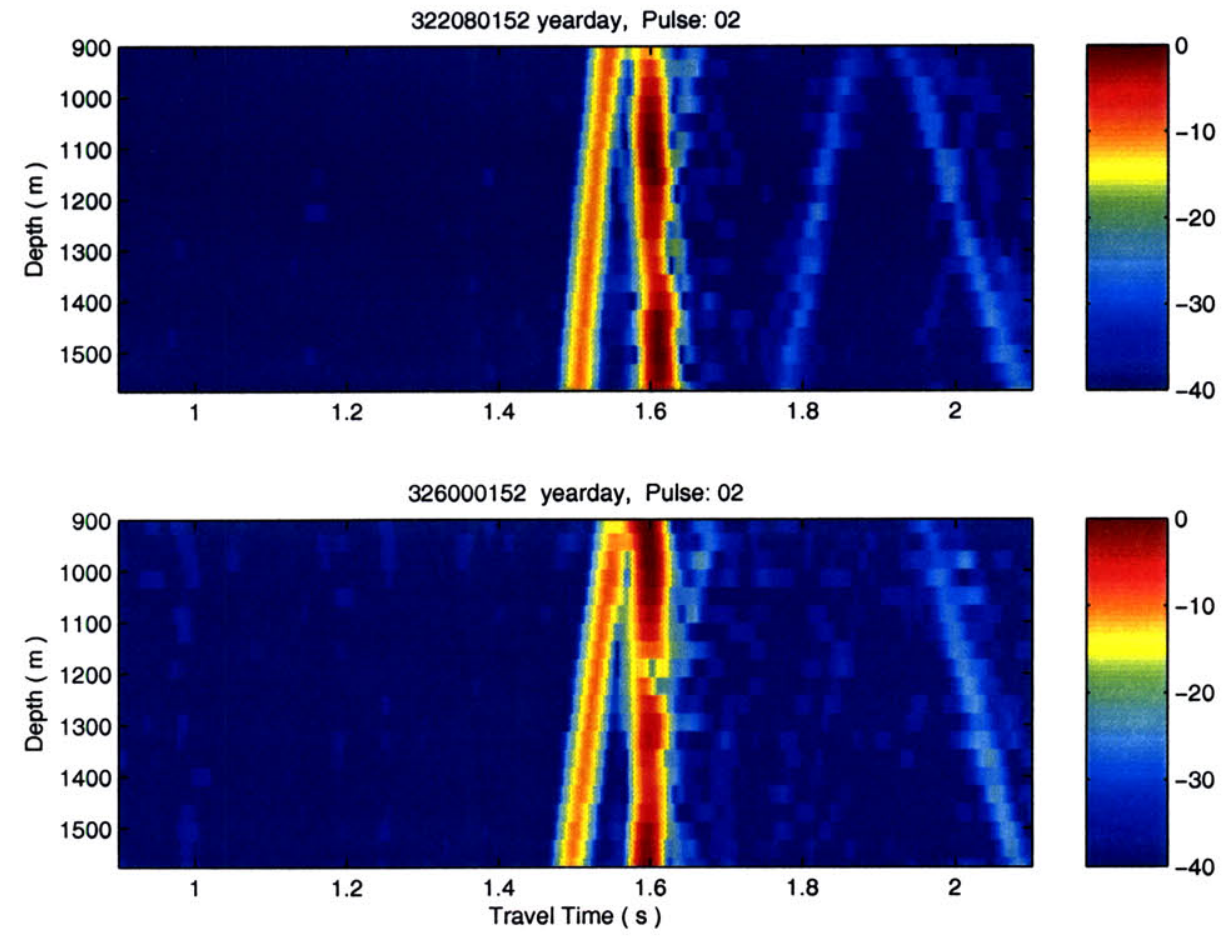

Figure 3-2: Two samples of sound pressure field recorded in the Near VLA. A 40 dB dynamic range is displayed in the figure. The travel time is relative time to the time when recording is started. The unit of plot is $\mathrm{dB}$ re max. 

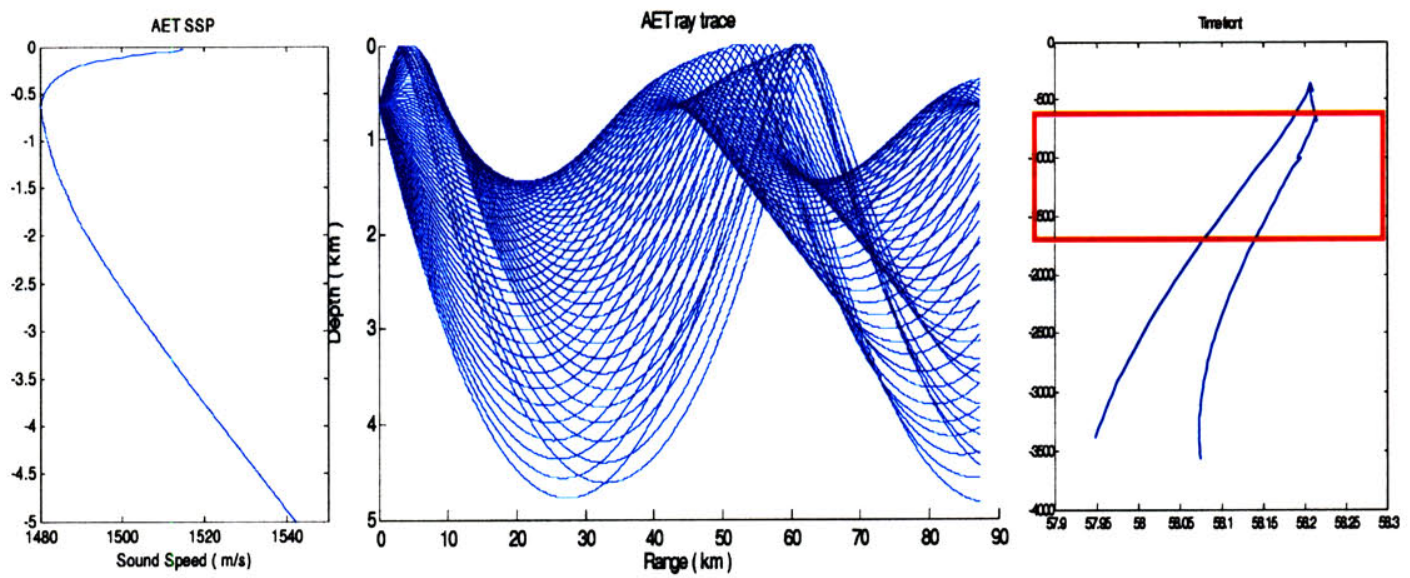

Figure 3-3: Range independent ray simulation result based on AET sound speed profile. The red frame on the time front shows the depth-range observed by the VLA.
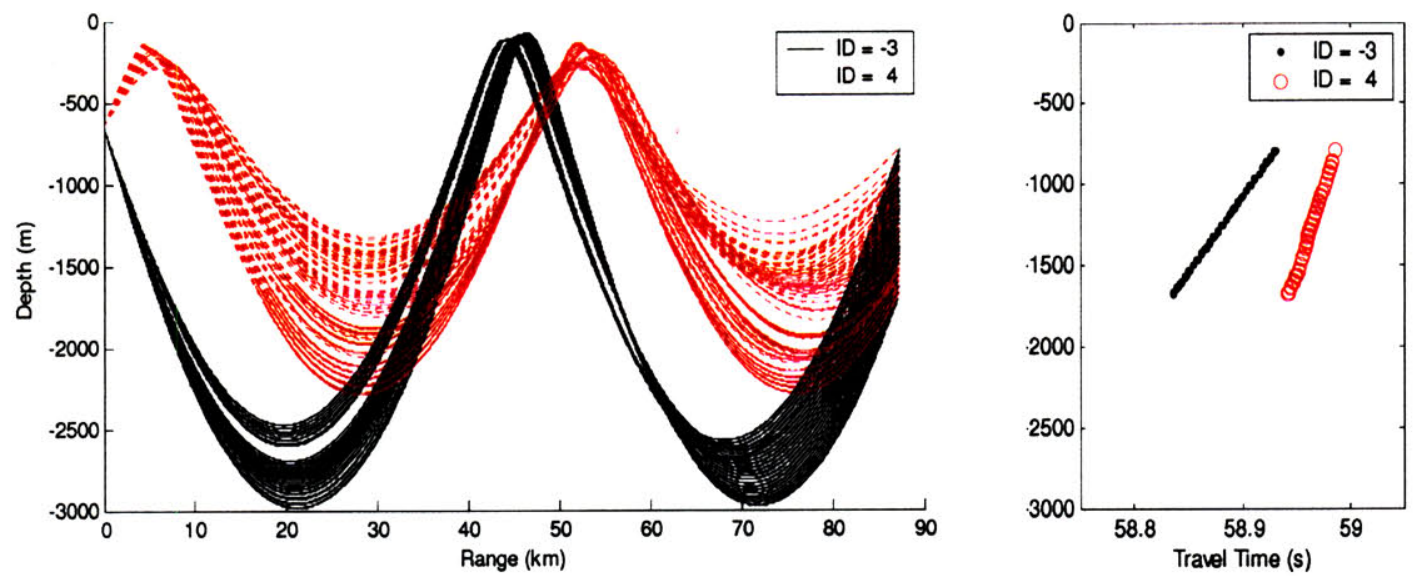

Figure 3-4: This plot is showing the eigen ray trace and time front for the $87 \mathrm{~km}$ range transmission and near VLA receiver configuration. The first two arrivals are corresponding the ray index -3 and ray index 4 .

carried out (Fig. 3-3). The ray calculation (Fig. 3-4) identifies the first arrival in Fig.3-3 with ray paths having initial downward angles and passing through 3 turning points; one UTP and two LTP's. This early arrival is then given the label ID -3 ; the minus sign to denote the initial downward ray angle, and the 3 to denote the total number of turning points. Similarly the ray calculation (Fig.3-4) identifies the second arrival with a group of rays with initial upwards angles and passing through 4 turning points; 2 UTP's and 2 LTP's. This arrival is then given the label ID +4 .

Table 3.2.1 gives information about the depths and ranges of the UTP's for the two arrivals. Wavefront ID -3 has all of its rays turning in a very small depth region between $90 \mathrm{~m}$ and $130 \mathrm{~m}$, and very close in range to the second UTP of ID $=4$. The mixed layer 
Table 3.1: Upper turning points depths and horizontal positions for ID's -3 and +4 .

\begin{tabular}{|c|c|c|c|}
\hline & Index -3 & \multicolumn{2}{|c|}{ Index 4 } \\
\hline & UTP & First UTP & Second UTP \\
\hline Depth Range(m) & $90-140$ & $225-350$ & $225-350$ \\
\hline Horizontal Position (km) & $44-46$ & $5-6$ & $52-55$ \\
\hline
\end{tabular}

during this experiment was very shallow of order $20-30-\mathrm{m}$ depth, so ID -3 is not impacted by the mixed layer, other than perhaps by diffractive, Fresnel zone effects. Wavefront ID -4 has a much more broad distribution of UTP's, spanning the depth range of 225-350-m.

\subsubsection{Arrival Selection and Processing}

Given the clear ray identification just described, we now seek to isolate the acoustic fluctuation associated with each ID. We do this by extracting the amplitude and travel time at the peak of the arrival at each depth. This is a fairly straight-forward procedure since the two wavefronts are well separated in time over most of the depth region. However, near the shallow end of the receiving array the arrivals do interface with one another and thus we implement a Maximum-likelihood estimator (See Appendix A) to extract the peak amplitude and travel time information of the two arrivals. The mathematical model for the received pressure signal at each hydrophone is

$$
r(T)=\sum_{j=1}^{2} A_{j} E\left(T-T_{j}\right) \cos \left(\omega\left(T-T_{j}\right)+\theta_{j}\right)+n(T)
$$

where $A_{j}, T_{j}$, and $\theta_{j}$ are the amplitude, travel time, and phase of the two arrivals that are to be estimated. The pulse center frequency $\omega$ is $75 \mathrm{~Hz}$. The function $E(T)$ is the pulse envelope, which is assumed known, and $n(T)$ is noise. The envelope of the pulse for a single path is estimated as follows. In regions where the two arrivals do not overlap, a travel time $T_{j}(z, t)$ is established from the peak of the envelope, and then the observed pulse envelopes are stacked and average so that

$$
E(T, I D)=\left\langle I(T, I D)>^{1 / 2}=\left(\frac{1}{N_{k} N_{i}} \sum_{k} \sum_{i} I\left(T-T_{j}\left(z_{k}, t_{i}\right)\right)\right)^{1 / 2}\right.
$$

where $I\left(T-T_{j}\left(z_{k}, t_{i}\right)\right)$ is the square of the absolute value of the complex envelope and $N_{k}$ 


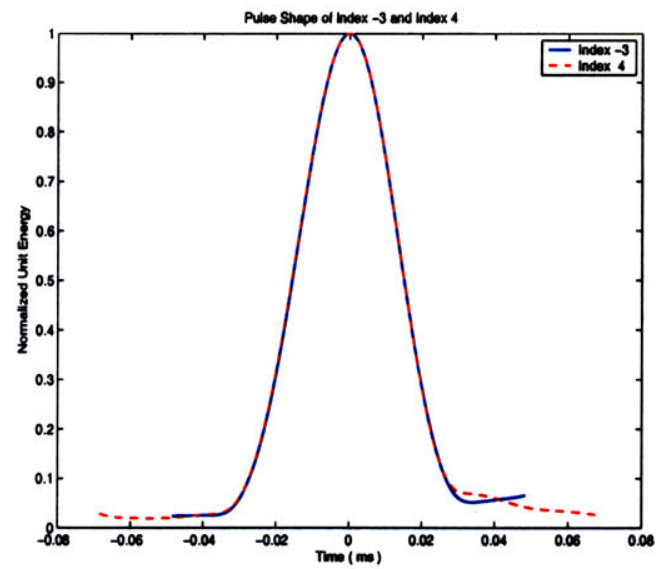

Figure 3-5: The estimated pulse shape for two arrivals.

and $N_{i}$ are the number of hydrophone depths used and number of transmissions used. In Eq. $3.1 I D=-3$ when $j=1$ and $I D=+4$ when $j=2$. Figure 3-5 shows the estimate of $\langle I(T, I D)\rangle$ for the two arrivals. Note that the pulse envelope $E(T, I D)$ is given a unit maximum value. Figure 3-6 shows the selected arrivals for the two wavefronts for one transmission, and one can see that where the two branches of the time front start to interfere the selected arrivals are no longer strictly at the maximum value of the timefront intensity.

The maximum-likelihood estimator was applied to all the pulses yielding a set of complex demodulates of the form

$$
\psi(z, t, I D)=A(z, t, I D) e^{i \phi(z, t, I D)}
$$

where $A(z, t, I D)$ are the arrival amplitudes from Eq. 3.2, and the phase is derived from the travel time and phase estimate in Eq. 3.1 giving $\phi(z, t, I D)=\theta_{j}-\omega T_{j}(z, t)$. The acoustic intensity variability is studied through the data $A(z, t, I D)$. However the data need to be re-normalized to remove any effects of hydrophone calibration variation and non-stationarity. Each hydrophone series is normalized to have unit mean intensity, that is

$$
<A^{2}>(z, I D)=<I>(z)=\frac{1}{N_{\tau} N_{l}} \sum_{k=1}^{N_{\tau}} \sum_{l=1}^{N_{l}} A^{2}\left(z, \tau_{k}, l ; I D\right)=1
$$

Figure 3-7 shows the RMS intensity as a function of depth for the two arrivals before normalization. There are some clear changes in the RMS intensity along these fronts before 


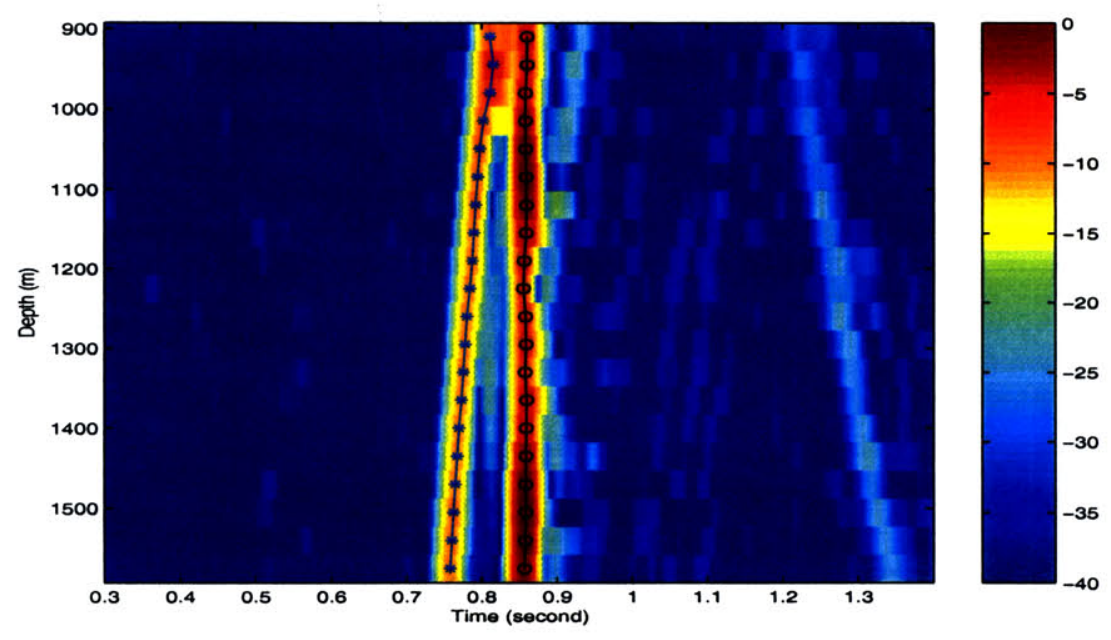

Figure 3-6: One example of resolving two arrivals by using maximum likelihood estimation method; This is transmission of year day 326, 10:00 of UTC time. The unit of plot is dB re $\max$.

normalization. Because of mooring and source motion issues which will be discussed in the upcoming sections the phase $\phi$ will also need to be corrected.

\section{Phase Unwrapping}

Over the consecutive 40 and 80 transmission intervals a smooth unwrapped phase as a function of depth and time can be formed. However, because of the 2 or 4 hour time gaps between the continuous transmission intervals phase cannot be tracked between these periods. Thus for notational simplicity we define the time coordinate $t$ as $t=\tau+\tau_{l}$ where the values of $\tau$ are only defined over the continuous transmission intervals (i.e. $0 \leq \tau \leq 39 \times 27.28$ $\mathrm{s}$, or $0 \leq \tau \leq 79 \times 27.28 \mathrm{~s} ; 0 \leq l \leq 39$, or $0 \leq l \leq 79)$. The variable $\tau_{l}$ then denotes the beginning time of each continuous set of transmissions. The notation for the complex demodulates then becomes

$$
\psi(z, \tau, l, I D)=A(z, \tau, l, I D) e^{i \phi(z, \tau, l, I D)}
$$

While the phase $\phi$ is defined in terms of the travel time we choose to remove this continuous variability and express $\phi$ only on the interval $0 \leq \phi \leq 2 \pi$. A smooth unwrapped phase function $\phi_{u}$ is determined from $\phi$ for each transmission sequence $\tau_{l}$. The unwrapping is carried out such that the mean square difference between gradients calculated from the wrapped 


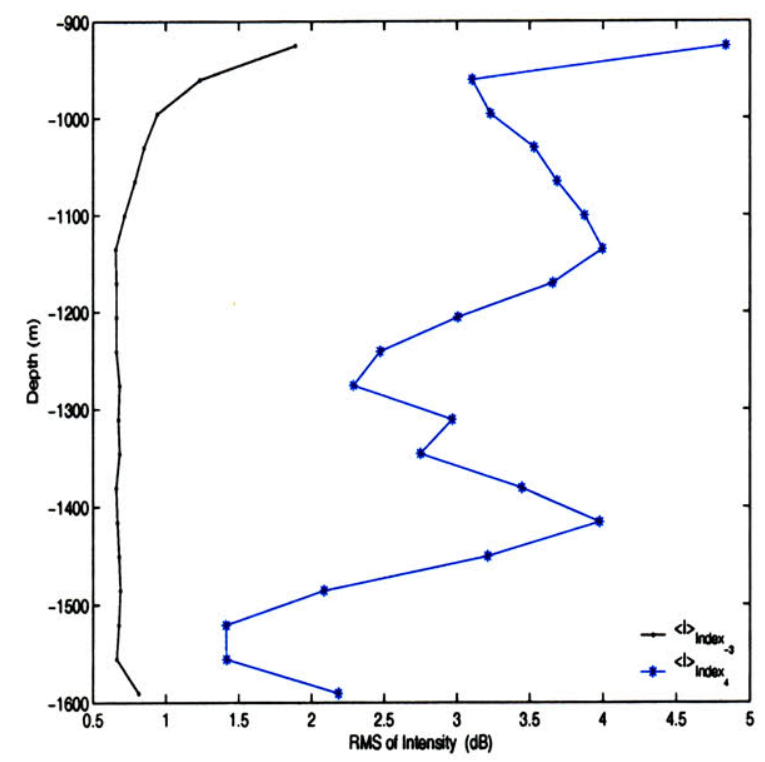

Figure 3-7: The RMS of intensity as function of depth for two arrivals.

and unwrapped phases are minimized[48]. Using this smoothness criterion, however, means that the unwrapped phase function is not necessarily different from the wrapped phase by an integer multiple of $2 \pi$, but we find that in practice the result are extremely good since the acoustic variability is rather weak. A discussion of the accuracy of the technique is given in Colosi et al 2005[17].

Figure 3-8 shows the wrapped and unwrapped phases (second and third rows) for the two arrivals for one transmission sequence. Wavefront ID -3 is shown on the left and the wrapped phases reveal a rather large vertical gradient of phase due to the tilting of the wavefront as it encounters the vertical receiving array. The other wavefront ID +4 on the other hand has very little vertical gradient and is clearly sweeping past the array at nearly normal incidence (see Fig. 3-2).

\section{Source and Mooring Motion}

Now the phase variability that we observe, for example in Fig. 3-8, is not all due to acoustic scattering. The source, while moored from FLIP, experienced some large horizontal deflection of order $100-\mathrm{m}$ with a timescale of hours to days. This motion was tracked every hour using a long baseline navigation system[83]. Similarly, the moored receiver array experienced horizontal deflections, as well as variable tilt and perhaps some bending. 
While the receiver array was designed with a long baseline navigation system to measure array deformation this system failed leaving only a single point on the mooring that was navigated[83]. Thus, because the source was only tracked every hour, and because the mooring only had one point of navigation twice an hour, we are unable to correct the unwrapped phases using this information; so we taken an empirical approach. To correct for mooring/source motion and mooring tilt, a least-square fit to the phase function is done to eliminate linear trends in both depth and time $\tau[17]$. Thus, for each transmission interval $\tau_{l}$ the linear trends are removed leading to a corrected phase function $\phi_{c}(z, \tau, l, I D)=$ $\phi_{u}(z, \tau, l, I D)-\phi_{m}(z, \tau, l, I D)$. In the bottom row of Fig. 3-8 the effects of removing the trends are shown, revealing much smaller phase variability. In the subsequent analysis, the corrected phase $\phi_{c}$ will be used to quantify acoustic variability.

\subsection{Observed Phase and Intensity Fluctuations}

In this section the corrected observed phase $\phi_{c}(z, \tau, I ; I D)$ and amplitude $A(z, \tau, l ; I D)$ are analyzed. First we present calculations of various moments of these fields, and then in the subsequent sections the frequency and vertical wavenumber spectra of these observations are examined.

\subsubsection{Moments}

The calculation of various moments of the phase and amplitude is somewhat complicated by the irregular sampling in the AET. As was previously mentioned, phase could only be quantified over the continuous transmission periods of roughly 20 and 40 minutes duration; that is to say we had no way of establishing the relative phase between two transmission intervals separated by 2 or 4 hours. Amplitude variations, however, do not have this problem. Thus, the moments we compute for phase will be for the two observation times of 20 and 4 minutes, while the moments we compute for amplitude will be for the 20 and 40 minute observation times as well as the whole 6 day observation time. 

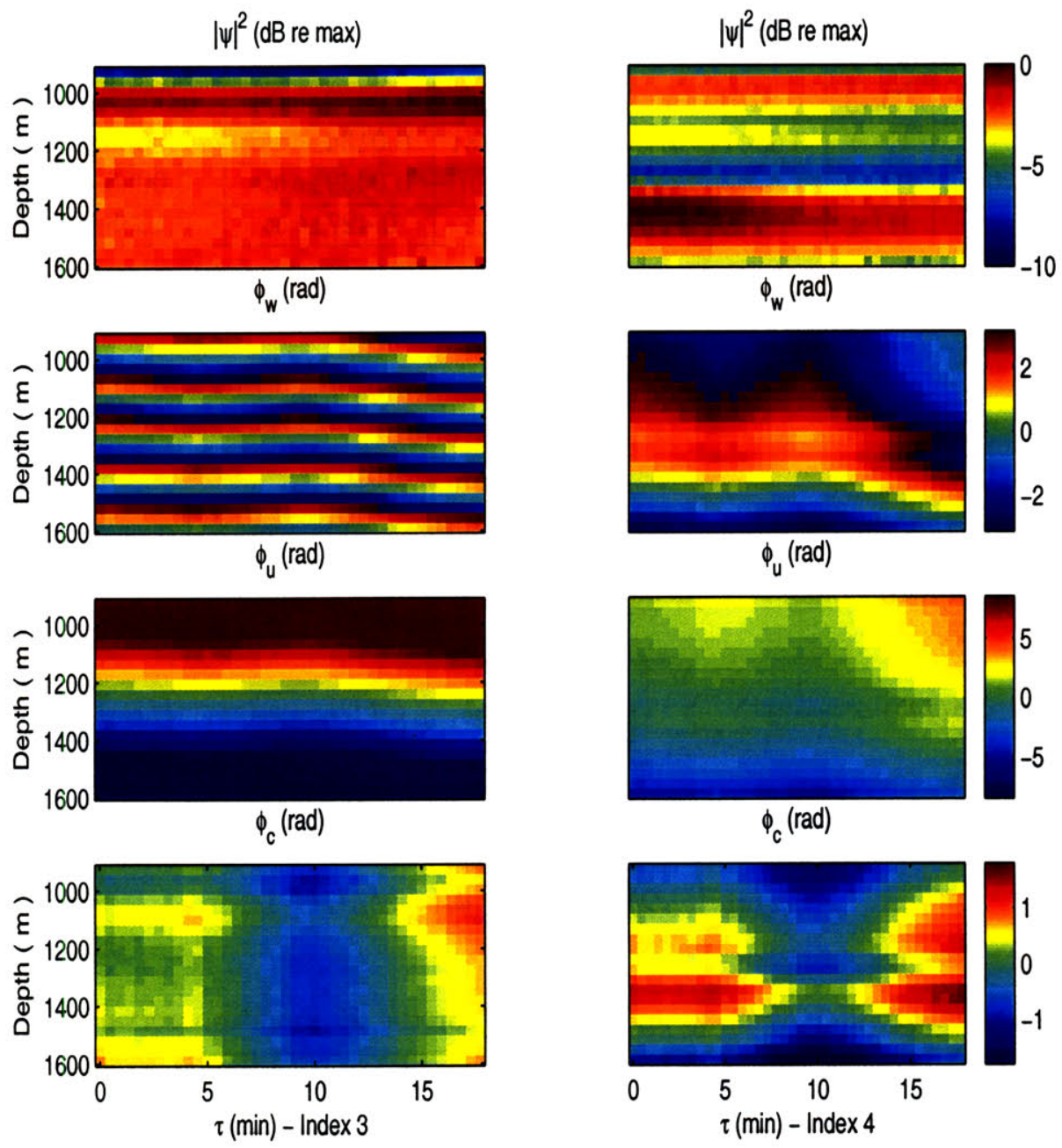

Figure 3-8: This figure displays the intensity and phase fluctuation as function of time ( $\mathrm{x}$ axis) and depth (y axis) of two wave fronts in 20-minute transmission. The first column corresponds to ID -3 arrival, the second column corresponds to ID 4 arrival. The first row is the intensity fluctuation in unit of $\mathrm{dB}$ re max. From the second to fourth row are wrapped phase, unwrapped phase and unwrapped phase with correction of mooring/source motion, respectively. 
Table 3.2: RMS phase, intensity, log-amplitude, and scintillation index of 20 and 40 minute transmission.

\begin{tabular}{|c|c|c|c|c|c|}
\hline & & $\begin{array}{c}<\phi^{2}>^{\frac{1}{2}} \\
(\mathrm{rad})\end{array}$ & $\begin{array}{c}<I^{2}>^{\frac{1}{2}} \\
(\mathrm{~dB})\end{array}$ & SI & $\left\langle\chi^{2}\right\rangle$ \\
\hline 20-Min & ID -3 & $0.4397 \pm 0.004$ & $0.2558 \pm 0.05$ & $0.0044 \pm 0.002$ & $0.0012 \pm 0.00013$ \\
\hline & ID 4 & $0.4406 \pm 0.002$ & $0.2581 \pm 0.07$ & $0.0054 \pm 0.0007$ & $0.0014 \pm 0.00018$ \\
\hline 40 -Min & ID -3 & $0.6101 \pm 0.01$ & $0.3820 \pm 0.10$ & $0.0089 \pm 0.001$ & $0.0023 \pm 0.0003$ \\
\hline & ID 4 & $0.6841 \pm 0.005$ & $0.4024 \pm 0.054$ & $0.0134 \pm 0.004$ & $0.0038 \pm 0.001$ \\
\hline 6 day & ID -3 & & $0.7972 \pm 0.07$ & $0.0439 \pm 0.013$ & $0.0095 \pm 0.002$ \\
\hline & ID 4 & & $3.0585 \pm 0.2$ & $0.427 \pm 0.05$ & $0.1338 \pm 0.016$ \\
\hline
\end{tabular}

Phase

The variance of phase is computed in the time direction and is averaged over all hydrophone depths $z$ and all transmission sequences $l$. To be specific we compute

$$
\begin{gathered}
\sigma_{\phi}^{2}(I D)=\frac{1}{N_{z} N_{l}} \sum_{k=1}^{N_{z}} \sum_{l=1}^{N_{l}}\left[\frac{1}{N_{\tau}} \sum_{j=1}^{N_{\tau}}\left(\phi_{c}\left(z_{k}, \tau_{j}, l, I D\right)-\bar{\phi}_{c}\left(z_{k}, l, I D\right)\right)^{2}\right] \\
\bar{\phi}_{c}\left(z_{k}, l, I D\right)=\frac{1}{N_{\tau}} \sum_{j=1}^{N_{t} a u} \phi_{c}\left(z_{k}, \tau_{j}, l, I D\right)
\end{gathered}
$$

where $N_{z}=20$, and $N_{l}=30$ and $N_{\tau}=40$ for the 20 minute observation time, and $N_{l}=3$ and $N_{\tau}=80$ for the 40 minute observation time. Table 3.2 shows the rms phase for the two arrivals for the two observation times. ${ }^{3}$ As would be expected, the variance increases for the longer observation time, since the phase has been able to change over more of the ocean's broadband of variability. Interestingly there is very little difference in the phase variability for the two arrivals. Apparently, the one shallow UTP for ID -3 has the same effect as two deeper UTP's for ID +4 . This is consistent with the results of Flatte and Stoughton (1988)[44] who find that phase variability for a timefront at fixed range is almost independent of wavefront ID.

\footnotetext{
${ }^{3}$ The error-bars are estimated by using the variations of estimates at each depth.
} 


\section{Amplitude}

Two different measures of amplitude variability were examined, which are scintillation in$\operatorname{dex}(\mathrm{SI})$, and variance of the $\log$ of intensity $\left(\iota=\ln A^{2}\right)$. These measures are defined as

$$
S I=\left\langle I^{2}\right\rangle /\langle I\rangle^{2}-1, \quad \sigma_{\iota}^{2}=\left\langle\iota^{2}\right\rangle-\langle\iota\rangle^{2}
$$

As before these moments are computed over specific observation times, but for amplitude we can now add the 6 day observation time. Specifically for the 6 day observation time we compute

$$
\begin{gathered}
\left\langle I^{2}\right\rangle(z ; I D)=\frac{1}{N_{\tau} N_{l}} \sum_{k=1}^{N_{\tau}} \sum_{l=1}^{N_{l}} A^{4}\left(z, \tau_{k}, l ; I D\right) \\
S I(I D)=\frac{1}{N_{z}} \sum_{j=1}^{N_{z}}\left\langle I^{2}\right\rangle(z) /\langle I\rangle^{2}(z)-1 \\
\sigma_{\iota}^{2}(I D)=\frac{1}{N_{z}} \sum_{k=1}^{N_{z}}\left[\frac{1}{N_{\tau} N_{l}} \sum_{l=1}^{N_{l}} \sum_{j=1}^{N_{\tau}}\left(\iota\left(z_{k}, \tau_{j}, l, I D\right)-\imath\left(z_{k}, I D\right)\right)^{2}\right] \\
\bar{\iota}\left(z_{k}, I D\right)=\frac{1}{N_{\tau} N_{l}} \sum_{l=1}^{N_{l}} \sum_{j=1}^{N_{\tau}} \iota\left(z_{k}, \tau_{j}, l, I D\right)
\end{gathered}
$$

For the shorter observation times the calculation proceeds along the lines as was described for phase. Specially we compute,

$$
\begin{gathered}
\left\langle I^{2}\right\rangle(z, l ; I D)=\frac{1}{N_{\tau}} \sum_{k=1}^{N_{\tau}} A^{4}\left(z, \tau_{k}, l ; I D\right) \\
\langle I\rangle(z, l ; I D)=\frac{1}{N_{\tau}} \sum_{k=1}^{N_{\tau}} A^{2}\left(z, \tau_{k}, l ; I D\right) \\
S I(I D)=\frac{1}{N_{z} N_{l}} \sum_{l=1}^{N_{l}} \sum_{j=1}^{N_{z}} \frac{\left\langle I^{2}\right\rangle\left(z_{j}, l\right)-\langle I\rangle^{2}\left(z_{j}, l\right)}{\langle I\rangle^{2}\left(z_{j}, l\right)} \\
\sigma_{\iota}^{2}(I D)=\frac{1}{N_{z} N_{l}} \sum_{l=1}^{N_{l}} \sum_{k=1}^{N_{z}}\left[\frac{1}{N_{\tau}} \sum_{j=1}^{N_{\tau}}\left(\iota\left(z_{k}, \tau_{j}, l, I D\right)-\tau\left(z_{k}, l, I D\right)\right)^{2}\right]
\end{gathered}
$$




$$
\tau\left(z_{k}, l, I D\right)=\frac{1}{N_{\tau}} \sum_{j=1}^{N_{\tau}} \iota\left(z_{k}, \tau_{j}, l, I D\right)
$$

Table 3.2 displays the estimates of $S I$ and $\sigma_{\iota}$ for the two arrivals. Again, we see that as observation time is increased the variance increases, with the largest increase occurring from the 40 minute observation time to the 6 day observation time. Comparing the two arrivals ID -3 and ID +4 , we see that the intensity fluctuations for the short observation times are very similar, but at the 6 day observation time ID +4 is significantly larger than ID -3 ; $0.8 \mathrm{~dB}$ rms compared to $3 \mathrm{~dB}$ rms! This important result suggests that the timescales of variability of the two arrivals are different, with the ID -3 showing much less low frequency variability than the ID +4 . This result will be examined in more detail when the frequency spectra are presented. In Fig.3-9, the time series of the two arrivals' intensity show that the second arrival (ID 4) has much more low frequency variability than the first arrival (ID -3) of these three hydrophones at different depths.

Finally calculation of the variance of $\log$-amplitude $(\chi=\ln A)$ reveal that $S I \simeq 4\left\langle\chi^{2}\right\rangle$ a result that is valid if the amplitudes $\mathrm{A}$ obey a log-normal distribution.

\subsubsection{Frequency Spectra}

Now we seek to decompose the temporal fluctuations of phase and amplitude in terms of the contributions at various frequencies. Regarding the observable of amplitude, we compute spectra for the $\log$-amplitude $\chi=\ln A$, since this is the quantity predicted by the MZ theory. The calculation is carried out by computing the frequency spectra at each

hydrophone depth and for each transmission sequence $l$, yielding $\hat{S}_{\phi, \chi}(\omega ; z, l, ; I D)$. These spectra are computed by Fast Fourier Transform (FFT) of either 40 or 80 time points with $27.28 \mathrm{~s}$ separation. Before FFT the data are de-trended, and windowed with a Hanning function. To obtain the final spectra estimate we average over all hydrophone depths and transmission sequence giving,

$$
S_{\phi, \chi}(\omega ; I D)=\frac{1}{N_{z} N_{l}} \sum_{l=1}^{N_{l}} \sum_{k=1}^{N_{z}} \hat{S}_{\phi, \chi}\left(\omega ; z_{k}, l ; I D\right)
$$

This procedure is carried out separately for the 20 minute and 40 minute observation times.

For the log-amplitude data we have information at longer timescales and thus lower 
Phone \# 1
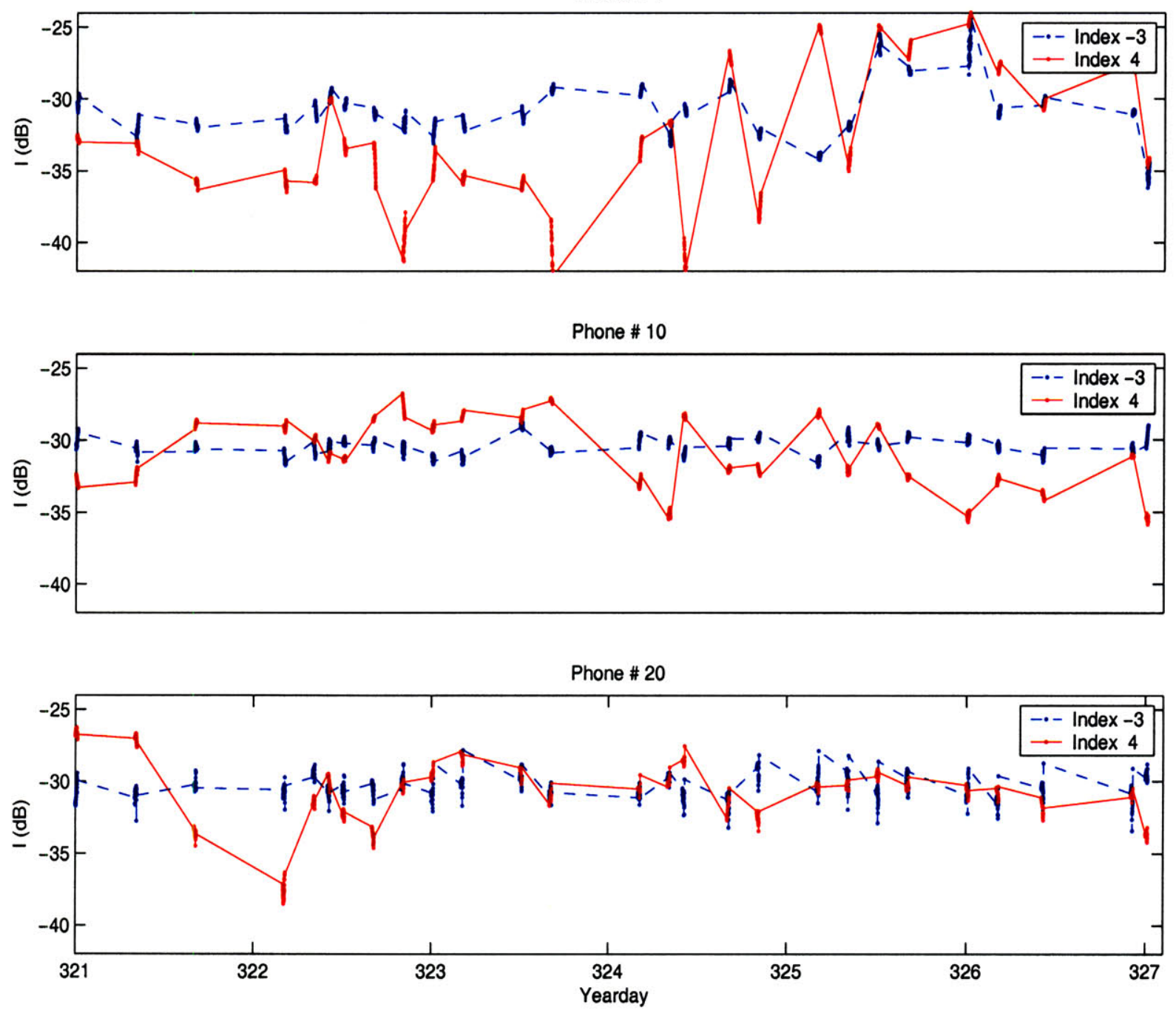

Figure 3-9: Figure shows the time series of intensity of hydrophone 1, 10 and 20 during 6 days observation period with the depths of around $900 \mathrm{~m}, 1250 \mathrm{~m}$ and $1600 \mathrm{~m}$, respectively. Note these are irregular sampled data of transmissions followed by different temporal gaps . The dot marks denote the sampling points. 
frequency. However, the data are irregularly sampled and thus this feature presents some difficulty in carrying out spectral analysis. After experimenting with several methods, we found that the best approach was to cubic spline interpolate the data onto a uniform grid with 4 hour time separation. Thus the 30-20 minute transmission periods over the 6 day experiment were interpolated onto 36 points to carry out the spectral analysis. Here the spectra were computed over the 36 sample, 6 day period for each hydrophone depth, and as before the data was de-trended and Hanning windowed before FFT. The final spectral estimates were obtained by averaging over depth.

Figure 3-10 shows the frequency spectra of phase and log-amplitude for the two wavefront arrivals. A maximum local buoyancy frequency of $6 \mathrm{cph}$ and the local Coriolis parameter $f$ are shown with vertical green lines; this is the internal wave frequency band. The phase spectra show nearly an $\omega^{-3}$ slope over the entire observed frequency range with a slight flattening of the slope at the buoyancy frequency. The observed spectral slope shows why the longer observation time of $\mathbf{4 0}$ minutes resulted in more phase variance relative to the 20 minute observation time. In addition the spectra for ID's -3 and +4 are very similar. Clearly the time sampling of the data does not allow us to separate the internal wave effect from other ocean process.

Regarding the spectra of log-amplitude, the high frequency end of the spectra show a flattening of the spectra at the highest frequencies which is likely due to noise. At about $10 \mathrm{cph}$ the spectral energy increases rapidly. Again, the increase in spectral energy with decreasing frequency explains why the 40 minute observation times had more variance than the 20 minute observation time. As with phase the high frequency end of the spectra are very similar for the two wavefronts, and the time sampling is really marginally sampling the internal wave band. The low frequency end of the spectra show very different behavior. Here wavefront ID -3 shows much less low frequency energy than ID +4 , thus confirming the result from the analysis of intensity variance. Some of the additional intensity variance comes from the internal wave band(i.e. $\omega>f)$, but some also comes from the so-called sub-inertial band (i.e. $\omega<f$ ).

\subsubsection{Vertical Wavenumber Spectra}

Finally, we examine the spatial structure of the phase and log-amplitude fluctuations using the vertical wavenumber spectrum. The wavenumber spectra are computed for each time $\tau$ 

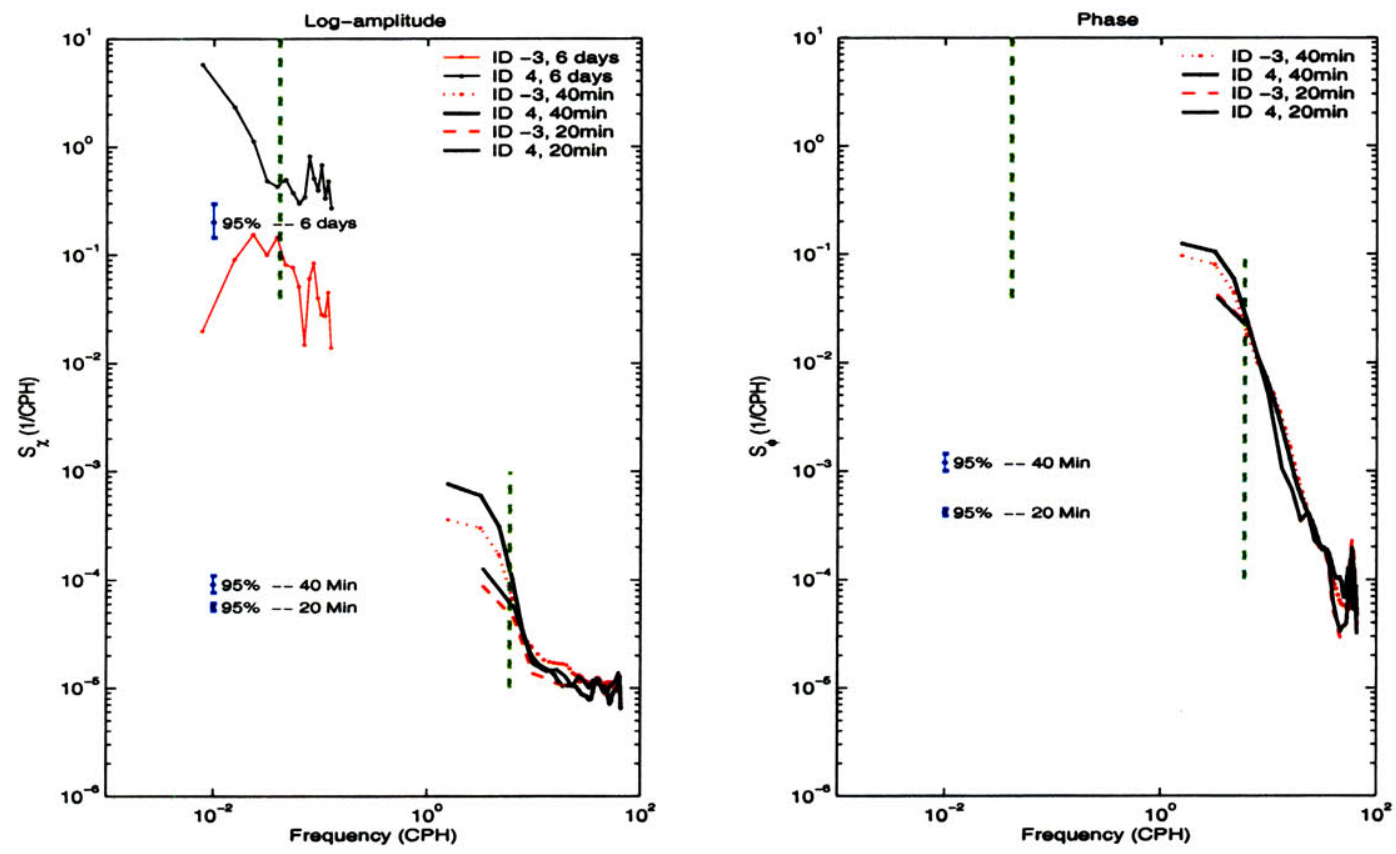

Figure 3-10: Frequency spectra of log-amplitude (left panel) and phase (right panel) of Index -3 (red) and Index 4 (black) arrivals. The green straight vertical line denotes the local buoyancy frequency around depth $160 \mathrm{~m}$, which is $6 \mathrm{CPH}$, and the local Coriolis parameter $f$. The gap in the observed log-amplitude spectra is due to irregular sampling.

and $l$, yielding an estimate $\hat{S}_{\phi, \chi}\left(k_{z} ; \tau, l ; I D\right)$. As before the vertical data are first de-trended and then Hanning windowed before FFT. To obtain the final spectrum an average is done over all times $\tau, l$ such that

$$
S_{\phi, \chi}\left(k_{z} ; I D\right)=\frac{1}{N_{\tau} N_{l}} \sum_{l=1}^{N_{l}} \sum_{j=1}^{N_{\tau}} \hat{S}_{\phi, \chi}\left(k_{z} ; \tau_{j}, l ; I D\right)
$$

Here both the 20 and 40 minute data are combined in the ensemble average. The resulting vertical wavenumber spectra are shown in Fig. 3-11. Regarding phase spectra, both arrivals show a roughly $k_{z}^{-3}$ shape, with the wavefront ID +4 revealing somewhat of a roll off at low wavenumber. The spectra of log-amplitude for the two arrivals, however, are markedly different. Wavefront ID -3 shows a rather flat wavenumber spectrum, while ID +4 shows a steeper spectrum with a roll off around $k_{z}=3 \mathrm{cpm}$. 

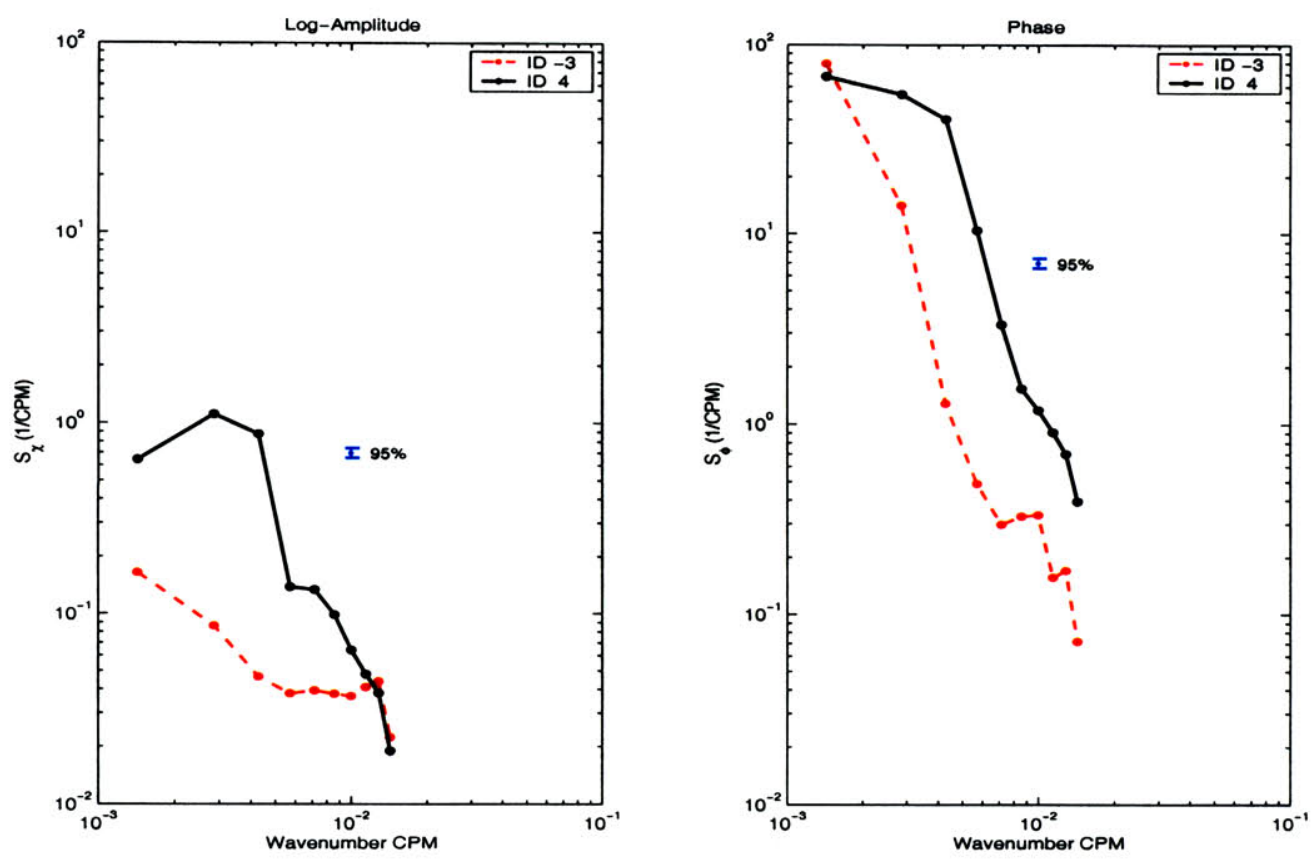

Figure 3-11: Vertical wavenumber spectra of phase (right panel) and log-amplitude (left panel) of Index -3 (red dot lines) and Index 4 (black solid lines) arrivals .

\subsection{Modeling}

Here we examine a hierarchy of three models to describe the acoustic variability seen in the AET. Here we assume that the fluctuating ocean sound speed field is dominated by internal waves obeying the Garrett-Munk internal wave spectrum which is validated in part in chapter 2. The simplest model is based on the Rytov theory of Munk and Zachariasen. The next model is a narrowband parabolic equation(PE) Monte Carlo model, and the most complex model is a fully broadband PE Monte Carlo Model.

\subsubsection{MZ Theory and Predictions}

The Rytov approximation, which is a smooth perturbation solution to the stochastic Helmholtz wave equation, is valid only in the weak scattering region for the wave propagating through random medium. It has been successfully applied to a case of electromagnetic wave propagation in the atmosphere when the scintillation index is less than 0.5(Flatté 1990)[59, 60]. AET experiment result shows scintillation index of the first arrival is 0.044 and second arrival is 0.4 , which means the scattering in the AET $87 \mathrm{~km}$ range transmission is very weak. Though, the criteria for Rytov theory being applied in the case of acoustic wave propagation 
in the ocean is still unknown, the AET experiment result suggests that the Rytov might be proper method in this special case. The detailed description of Rytov theory is presented in the Appendix B.

We find that the spectrum of phase and log-amplitude is written as an integral along a ray path $z_{r}(x)$ of the form,

$S_{\phi, \chi}\left(R, \omega, k_{z}\right)=\pi k_{0}^{2} \int_{\Gamma} d s \Phi\left(0, k_{\perp}\left(\omega, k_{z}\right) ; z\right)\left[1 \pm \cos \left(\frac{k_{z}^{2} R_{f z}^{2}(x)}{2 \pi}\right)\right] H\left[\omega-\omega_{L}\left(z_{r}(x)\right)\right] H\left[N\left(z_{r}(x)\right)-\omega\right]$

where the plus sign refers to the spectrum of phase and the minus sign refers to the spectrum of log-amplitude $\chi, k_{0}$ is a reference acoustical wavenumber. The integral involves two terms, the first of which is the spectrum of sound speed fluctuations evaluated for internal wave wavenumbers that are perpendicular to the sloping ray, $\Phi\left(0, K_{\perp}\left(\omega, k_{z}\right) ; z\right)$ and the second term in square brackets which is a diffraction term involving the vertical Fresnel zone $R_{f z}$. We discuss the spectral term first.

A unit vector in the direction of the ray with slope $\theta_{r}$ is $\left(\cos \theta_{r}, 0, \sin \theta_{r}\right)$ and thus the component of the internal wave wave-vector perpendicular to the ray is $\vec{k}_{\perp}=\left(-k_{z} \tan \theta_{r}, k_{y}, k_{z}\right)$. The internal wave dispersion relationship in the WKB limit is,

$$
k_{h}=\left(k_{x}^{2}+k_{y}^{2}\right)^{1 / 2}=\left(k_{z}^{2} \tan ^{2} \theta_{r}+k_{y}^{2}\right)^{1 / 2}=\left(\frac{k_{z}}{N(z)}\right)\left(\omega^{2}-f^{2}\right)^{1 / 2}
$$

where $f$ is the Coriolis parameter, $\omega$ is the internal wave frequency, and $N(z)$ is the buoyancy frequency profile. Thus solving for $k_{y}$ we obtain,

$$
\begin{gathered}
k_{y}= \pm\left(\frac{k_{z}}{N(z)}\right)\left(\omega^{2}-\omega_{L}^{2}\right)^{1 / 2} \\
\omega_{L}^{2}=f^{2}+N^{2}(z) \tan ^{2} \theta_{r}
\end{gathered}
$$

Thus, it is seen that for internal wave frequencies less than $\omega_{L}$ the dispersion relation cannot be satisfied to yield a real value for $k_{y}$; Hence, in this approximation, internal waves with frequencies less than $\omega_{L}$ do not locally interact with the acoustic field. To make this effect explicit and to impose the internal wave cut off for frequencies greater than $N(z)$, Heavyside functions are placed in Eq. 3.20. In terms of the Garrett-Munk internal wave spectrum, the evaluation of $\Phi$ using the perpendicular wavenumbers can be written in terms 
of the frequency-vertical wavenumber spectrum which has the form (See Appendix B),

$$
\begin{aligned}
& \Phi\left(0, k_{\perp}\left(\omega, k_{z}\right) ; z\right)=\Phi\left(0, k_{\perp}\left(k_{y}, k_{z}\right) ; z\right) \frac{d k_{y}}{d \omega} \\
&=\frac{\mu_{0}^{2} N^{3}}{N_{0}^{3}} \frac{8}{\pi^{3}} \frac{k_{z *}}{k_{z}\left(k_{z}^{2}+k_{z *}^{2}\right)} \frac{N(z) f}{\omega^{3}}\left(\frac{\omega^{2}-f^{2}}{\omega^{2}-\omega_{L}^{2}}\right)^{1 / 2}, \omega \geq \omega_{L} \\
& k_{z *}(z)=\frac{\pi j_{*} N(z)}{N_{0} B}, N_{0} B=\int_{0}^{D} N(z) d z
\end{aligned}
$$

Here $\mu_{0}^{2}=6.26 \times 10^{-8}$ is a reference fractional sound speed variance, and for the buoyancy frequency profile used here (Fig. 3-1) we have $N_{0} B=10.3(\mathrm{rad}-\mathrm{m} / \mathrm{s}) . D$ is the water depth. The first factor in Eq. 3.26 is in fact the fractional sound speed variance as a function of depth, which scales as $N^{3}[71]$, and for the GM spectrum $j_{*}=3$. Note that the spectrum without the perpendicular wavenumber constraint is

$$
\Phi\left(\omega, k_{z} ; z\right)=\frac{\mu_{0}^{2} N^{3}}{N_{0}^{3}} \frac{4 f}{\pi^{2}} \frac{k_{z *}}{k_{z}^{2}+k_{z *}^{2}} \frac{\left(\omega^{2}-f^{2}\right)^{1 / 2}}{\omega^{3}}
$$

Equation 3.26 shows that at large frequency and mode number the spectrum scales as $\omega^{-2}$ and $k_{z}^{-2}$. The spectrum under the perpendicular wavenumber constraint (Eq. 3.24), however, scales as $\omega^{-3}$ and $k_{z}^{-3}$ thus adding an additional $k_{z}^{-1}$ and $\omega^{-1}$ dependence to the spectrum.

The second term in Eq. 3.20 within the square brackets is often termed the Fresnel filter and it can be considered a weighting function on the spectrum controlling the spectral contributions to the variances $\left\langle\chi^{2}\right\rangle$ and $\left\langle\phi^{2}\right\rangle$ at each wavenumber $k_{z}$. The computation of the Fresnel zone $R_{f z}\left(z_{r}(x)\right.$ ) is well known (See Flatté 1983)[40] and is summarized in Appendix C. The physical significance of the Fresnel zone, is that it is the scale at which scattering can cause interference[40]. The product $k_{z} R_{f z}$, a ratio of medium sound speed scales to acoustic scales, measures the relative effects of diffraction (see Flatté et al 1979 and the discussion of the diffraction parameter $\Lambda$ ); small/large $k_{z} R_{f z}$ means small/large diffraction. Note also that the Fresnel filter as a function of $k_{z}$ has its first maximum at $k_{z}=0$ for phase and $k_{z}=\sqrt{2} \pi / R_{f z}(x)$ for log-amplitude. Since the internal wave spectrum evaluated at the perpendicular wave-number goes approximately like $k_{z}^{-3}$, then the largest contributions to the variance of phase will come from the large scales (i.e. small $k_{z} \simeq 0$ ) while the largest contributions to the log-amplitude variance will come from the scales near 
Fresnel zone. The interpretation just given is for the case where there is no wave-guide, and thus only applies locally along the ray in our approximation. In the wave-guide case the total effect is the integral over the entire ray path and thus represents the contribution from not only the Fresnel factors, but also the strength of the sound speed fluctuations as a function of depth, and also the low frequency cutoff factor $\omega_{L}$.

Figure 3-12 shows numerical evaluations of $z_{r}(x), R_{f z}(x)$, and $\omega_{L}(x)$, and for two eigenrays with ID's -3 and +4 from the AET environment (Fig.3-1). Here $N_{0} B$ has a value of $10.3 \mathrm{rad} \mathrm{m} / \mathrm{s}$, and launch angles for the eigenrays are $-9.8^{\circ}$ and $5.3^{\circ}$ for ID's -3 and +4 respectively. Regarding the Fresnel zones for the two arrivals, it is seen that both functions roughly follow the envelope for the constant background sound speed case (i.e. $\left.R_{f z}^{2}=\lambda x(R-x) / R\right)$, but the +4 ID has more structure than the -3 ID. This is because caustics are zeros of $R_{f z}$ and having gone through more turning points ID +4 has gone through more caustics than ID -3. With respect to the relative size's of the Fresnel zones ID +4 shows a larger maximum Fresnel zone (600-m) compared to ID -3 's value of $400-\mathrm{m}$. Thus ID +4 may have contribution to the log-amplitude variance from slightly larger scales than ID -3. However, of critical importance is the behavior of $\omega_{L}$ along the ray path. For ID -3 $\omega_{L}$ rises to significantly larger values than ID +4 , and these large values extend over a significant region around the upper turning point. Thus ID -3 is expected to have significant depletion of low frequency variability compared to +4 , which is exactly the result from the AET observations.

Figure 3-13 shows the model log-amplitude and phase frequency spectra computed from numerical integration of Eq. 3.20, using Eq.3.24, and the vertical wavenumber contribution in the model are integrated out according to

$$
S_{\phi, \chi}(R, \omega)=\int_{0}^{\infty} S_{\phi, \chi}\left(R, \omega, k_{z}\right) d k_{z}
$$

Several points are noteworthy. As expected, the spectra cut off at the critical frequencies of $f$ and $N_{\max } \simeq 6 \mathrm{cph}$. Second, the model log-amplitude spectra, like the observations (see Fig 3-9), show that ID +4 has significantly more low frequency variability compared to ID -3. Interestingly the phase spectra also show the low frequency enhancement for ID +4 . Comparing variances for log-intensity the model gives $\sigma_{\ln I}=2.0 \mathrm{~dB}$ and $0.8 \mathrm{~dB}$ for ID's +4 and -3 , while the observations have $3.1 \mathrm{~dB}$ and $0.80 \mathrm{~dB}$; a rather favorable comparison. 

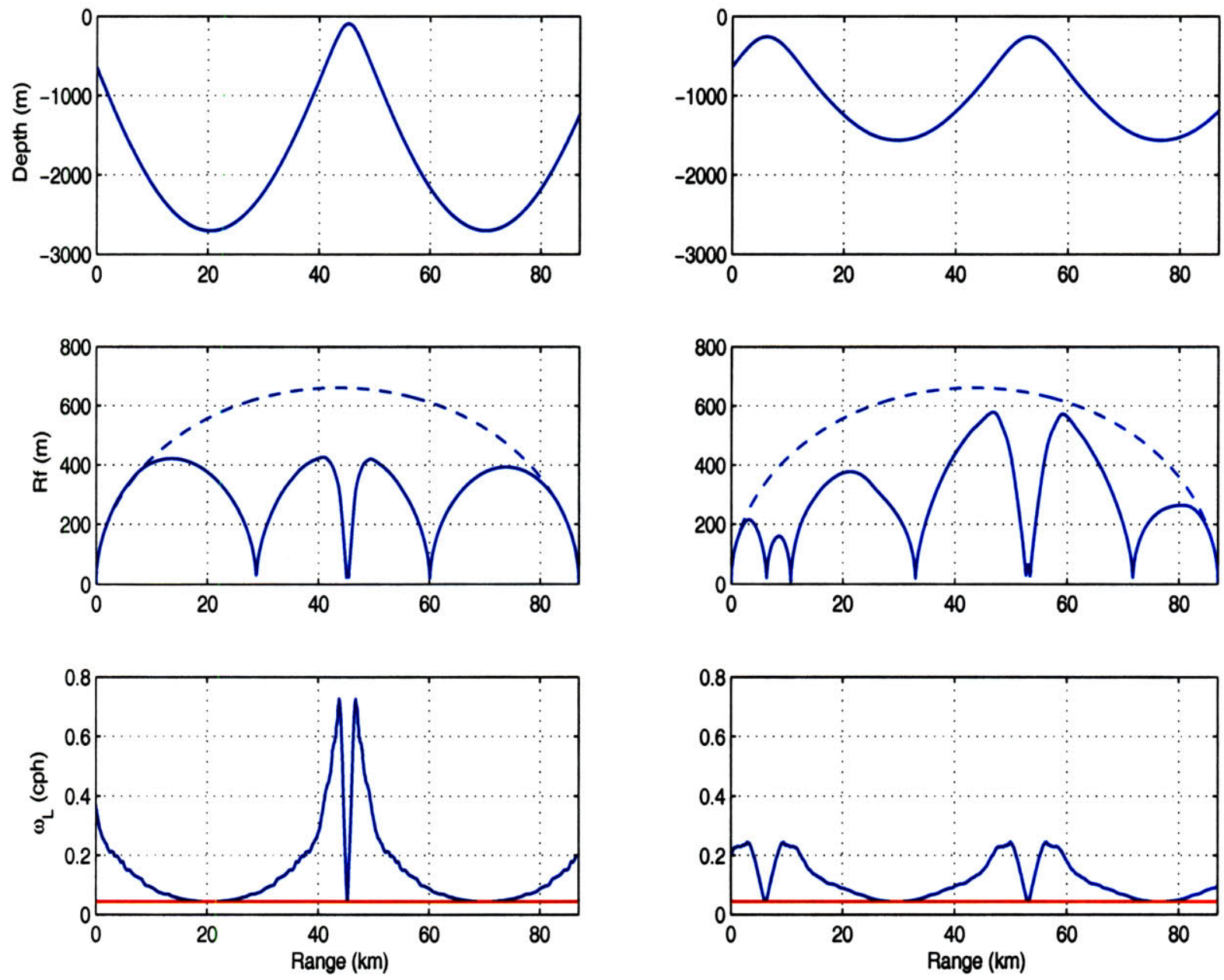

Figure 3-12: Raypaths (upper), Fresnel zone $R_{f z}(x)$ (middle), and low frequency cutoff $\omega_{L}(x)$ (lower) for two eigenrays with ID's -3 (left) and +4 (right). In the middle panel the Fresnel zone for constant background sound speed(dash) is shown for reference. In the lower panel the Coriolis frequency $f$ is shown with horizontal red line. 

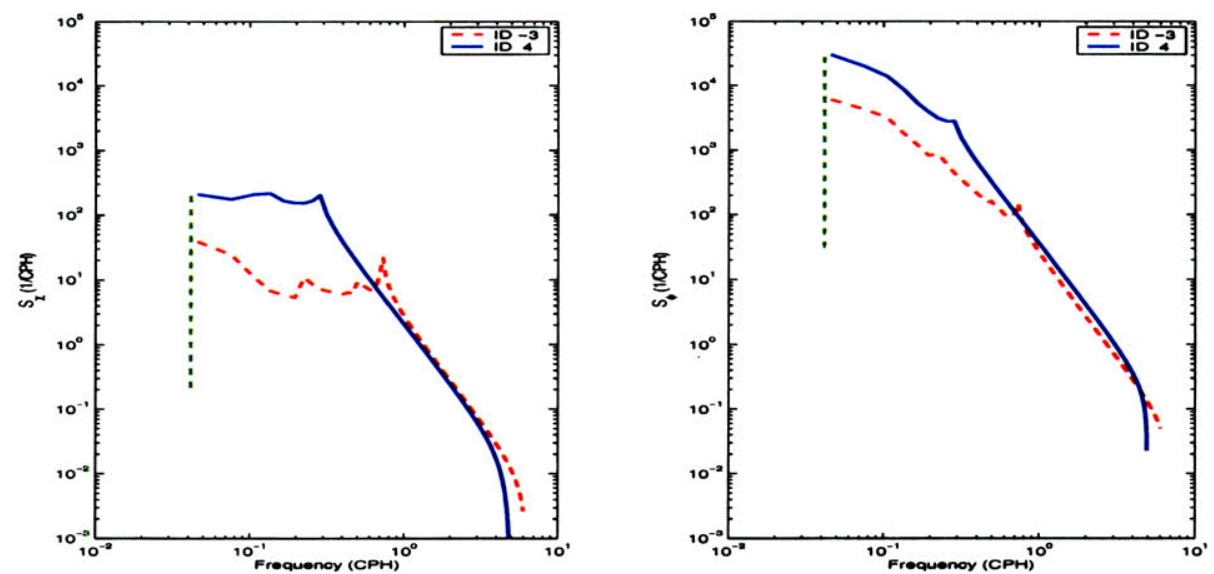

Figure 3-13: Rytov prediction of frequency spectrum of wave fronts' phase (left panel) and log-amplitude (right panel) for a $75 \mathrm{~Hz} \mathrm{cw}$ signal propagation through a $87 \mathrm{~km}$ range. The two arrivals correspond to ID -3 (red dash) and ID +4 (blue solid ) arrivals. $N_{0}=6.12$ (cph), is denoted by vertical straight green line. The variance of phase and log amplitude are respectively $<\chi^{2}>_{I D 3}=0.017,<\chi^{2}>_{I D 4}=0.102,<\phi^{2}>_{I D 3}=0.996$ and $<\phi^{2}>_{I D 4}=$ 4.21 .

Third, the shape of the spectra reveal distinct high and low frequency regions; in the high frequency region the spectra have very nearly an $\omega^{-3}$ form, and in the low frequency region the spectra are rather flat. Separating these regions is a cusp-like feature which occurs at frequencies of roughly $0.72 \mathrm{cph}$ and 0.28 for ID's -3 and +4 respectively. Refering to Fig 3-12 this transition frequency corresponds to the peak value of $\omega_{L}$ near the ray upper turning point.

Figure 3-14 shows the modeled vertical wavenumber spectra for phase and log-amplitude, and here these spectra are obtained by integrating the two-dimentional spectra over frequency,

$$
S_{\phi, \chi}\left(R, k_{z}(j)\right)=\int_{f}^{N_{\max }} S_{\phi, \chi}\left(R, \omega, k_{z}(j)\right) d \omega
$$

For this calculation we have $N_{\max }=6 \mathrm{cph}$. At large vertical wavenumber both spectra of log-amplitude and phase show the distinct $k_{z}^{-3}$ shape, and at low wavenumber the logamplitude spectrum rolls off at roughly $k_{z *}$ while the phase spectrum shows only a subtle change in slope.

\subsubsection{Narrowband Model}

In comparing acoustic fluctuation theories, like the MZ theory, to observations, the (expected) short-comings of the theory can be broken down into two categories; those that in- 

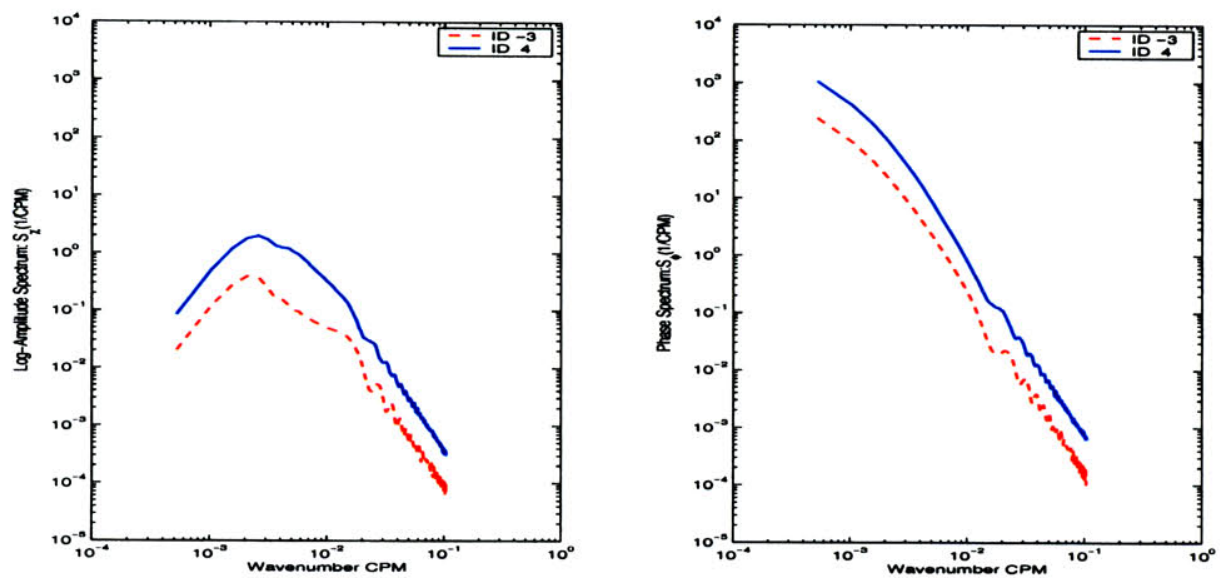

Figure 3-14: Rytov prediction of vertical wavenumber spectrum of wave fronts' phase(left panel) and log-amplitude (right panel) for a $75 \mathrm{~Hz} \mathrm{cw}$ signal propagation through a $87 \mathrm{Km}$ range. The two arrivals correspond to ID -3 (red dash) and ID +4 (blue solid ) arrivals.

volve issues of acoustic wave propagation, and those that involve the modeling of the ocean environment, like the internal wave field. For the former topic, there are three main acoustic issues with the MZ theory that we will consider here. First, the MZ theory is a perturbation approach and thus higher order terms may be important. Second, there is the assumption that the ray path has very little curvature, and thus the straight ray result is applied locally; and assumption that is clearly violated exactly at the upper turning point, where the slope is zero and the curvature is maximum. Flatté (1988)[44] and Colosi(1999)[20] have shown that this approximation places too much of the scattering strength near the ray upperturning point. Thirdly, the MZ theory is inherently narrowband, and thus issues of signal bandwidth in the observations needs to be addressed. To address these three issues, we have carried out parabolic equation numerical simulations of acoustic propagation through random fields of internal wave induced sound speed fluctuations. In the following sections, narrowband simulations are discussed.

\section{Simulation of Internal Waves}

The validity of Rytov solution with the random sound speed background, is checked by running numerical simulation using time-consuming Monte Carlo methods. The simulations are arranged to assure weak scattering so that the Rytov approximation may be used to predict both acoustic log-amplitude and phase fluctuation statistics. In this work, only internal waves are taken into consideration as the main random source induce the sound 
fluctuation in the ocean. Two-dimensional realizations of internal wave displacement fields are obtained using direct numerical simulation. These fields are evolved in time and are converted to sound-speed fluctuations using standard methods. Time series of acoustic signals at different depths are obtained using the parabolic equation (PE) method to propagate acoustic waves through the internal wave fields.

We represent the ocean sound-speed field as the sum of a deterministic mean profile plus a stochastic perturbation induced by internal waves, and the form of this ocean model is

$$
c(x, y, z, t)=c_{0}[1+U(z)+u(x, y, z, t)]
$$

where $U(z)$ is a deterministic function representing a range-independent background sound channel and $u(x, y, z, t)$ is a zero-mean stochastic perturbation representing the sound-speed fluctuations caused by internal waves. $U(z)$ is on the order of $10^{-2}$ whereas $u(x, y, z, t)$ is on the order of $10^{-4}[58]$. For small internal-wave displacements the form of $u(x, y, z, t)$ is

$$
u(x, y, z, t)=-\frac{g G}{\rho_{0}} \frac{\partial \rho}{\partial z} \zeta(x, y, z, t)
$$

where $G$ is of order unity and relates the relative potential sound-speed gradient to the buoyancy profile

$$
\frac{1}{c_{0}}\left(\frac{\partial c}{\partial z}\right)_{p}=\left(\frac{\partial U(z)}{\partial z}\right)_{p}=G N^{2}(z)
$$

where $(\partial U(z) / \partial z)_{p}$ is the fractional potential gradient of sound speed and $\zeta(x, y, z, t)$ is the vertical displacement caused by internal waves. Figure 3-15 shows the average sound-speed profile and the average smoothed buoyancy profile calculated from CTD casts obtained during the AET experiment. The internal wave simulation in the following sections utilizes these profiles.

The numerical technique used to simulate the ocean internal wave displacement has been widely discussed in several papers[18]. In general, the internal-wave displacement is modeled as a superposition of linear modes with random phases and amplitude. The wave amplitudes are chosen to match the energy distribution in wavenumber-frequency space of the GM internal-wave spectrum. 

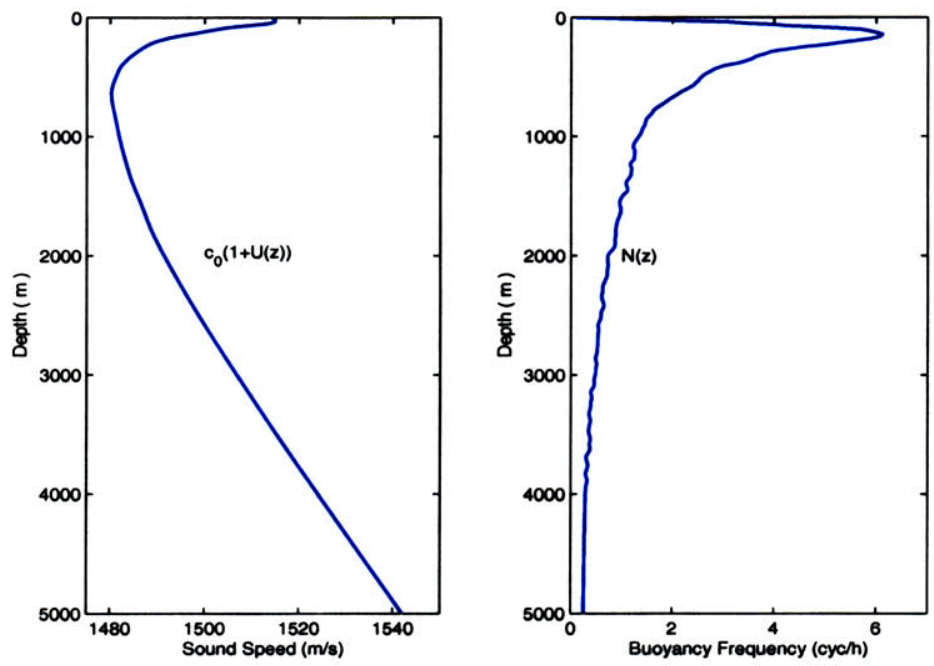

Figure 3-15: Estimates of the sound-speed profile and the buoyancy profile during the AET experiment. The buoyancy profile was smoothed

\section{Acoustic Simulation: Parabolic Equation}

The standard parabolic equation (SPE) and split-step Fourier algorithm are used to calculate the solution and simulate acoustic propagation. In the Appendix D, the detail of this method is presented. The split-step marching solution used in this chapter is:

$$
\psi\left(r_{0}+\Delta r, z\right)=e^{\frac{i k_{0}}{2}\left[n^{2}\left(r_{0}, z\right)-1\right] \Delta r} \mathcal{F}^{-1}\left\{e^{-\frac{i \Delta r}{2 k_{0}} k_{z}^{2}} \mathcal{F}\left\{\psi\left(r_{0}, z\right)\right\}\right\}
$$

where $\mathcal{F}$ is Fourier transform, $k_{0}=\omega / c_{0}$, and $\omega$ is frequency. This method is very efficient and accurate for the deep ocean and horizontal propagation as long as the validity of the approximations made are satisfied.

The total broad-band wave field in the time domain is obtained by Fourier synthesis. A generalized Gaussian source with both variable beamwidth and beam tilt is used as initial condition:

$$
\psi(0, z)=\sqrt{k_{0}} \tan \theta_{1} \exp \left(-\frac{k_{0}^{2}}{2}\left(z-z_{s}\right)^{2} \tan ^{2} \theta_{1}\right) \exp \left(i k_{0}\left(z-z_{s}\right) \sin \theta_{2}\right)
$$

where $\theta_{1}$ is the halfwidth of the source aperture ${ }^{4}$, and $\theta_{2}$ is the beam tilt with respect to the horizontal, measured positive downward.

\footnotetext{
${ }^{4} \mathrm{As}$ is customary for Gaussian beams, the beamwidth is defined as the $1 / e$-decay point perpendicular to the axis, and angle associated with this particular beamwidth is denoted by $\theta_{1}$.
} 
The boundary conditions on the acoustic propagation are a reflecting ocean surface and an absorbing ocean bottom. The reflecting ocean surface is modeled numerically using an image ocean.

In order to model an absorbing bottom a gradual loss of amplitude is imposed on $\psi(\mathbf{r}, \mathbf{t})$ as $z$ approaches the ocean bottom. The functional form of the imposed loss at each step is

$$
L(z)=\exp \left[-\beta d x \times \exp \left(-\left(\frac{z-z_{b}}{\alpha z_{b}}\right)^{2}\right)\right]
$$

with $\beta=0.04$ and $\alpha=0.05$. This form effectively stops any acoustic energy from penetrating about $\alpha \times z_{b}=250 \mathrm{~m}$ above the ocean bottom $z_{b}\left(z_{b}=5500 \mathrm{~m}\right.$ in our study.)[58].

\section{Narrow-Band Numerical Simulation Results with Waveguide}

The random ocean sound-field is simulated by adding a deterministic mean profile with stochastic perturbation induced by internal wave. The sound speed profile has been already shown in Fig.3-15.

First, a slice of the internal wave field is generated at time $t_{i}$. Then the parabolic equation method is used to propagate the sound wave energy through this internal wave field. The sound pressure field at the receiver range for time $t_{i}$ is recorded. In the next step, the internal wave field will evolve in time following the internal wave dispersion relationship. Then the sound wave energy propagates through this evolved internal wave field again. By doing this step by step, the sound wave fluctuation field will be obtained by propagating the sound through this simulated "real" random fluctuated ocean. This is the basic idea of Monte Carlo simulation, i.e. "frozen field approximation".

In Fig. 3-16, a realization of internal wave displacement is shown for a slice with range of $100 \mathrm{~km}$ and depth of $5 \mathrm{~km}$. As we can see, the internal wave field is inhomogeneous in the vertical direction and anisotropic with the fluctuations elongated in the horizontal direction. This internal wave field evolves in time with time step of 120 seconds. A fixed position sampling of the evolving internal wave field is shown in Fig. 3-17, which is about 2.8 days period of evolution with 120 seconds interval for each time step. The corresponding spatial and temporal spectra of the internal wave fields are shown in the following figures: Fig. 3-18 and Fig. 3-19.

A generalized Gaussian source with small launch angle is sent out at certain depth (650 


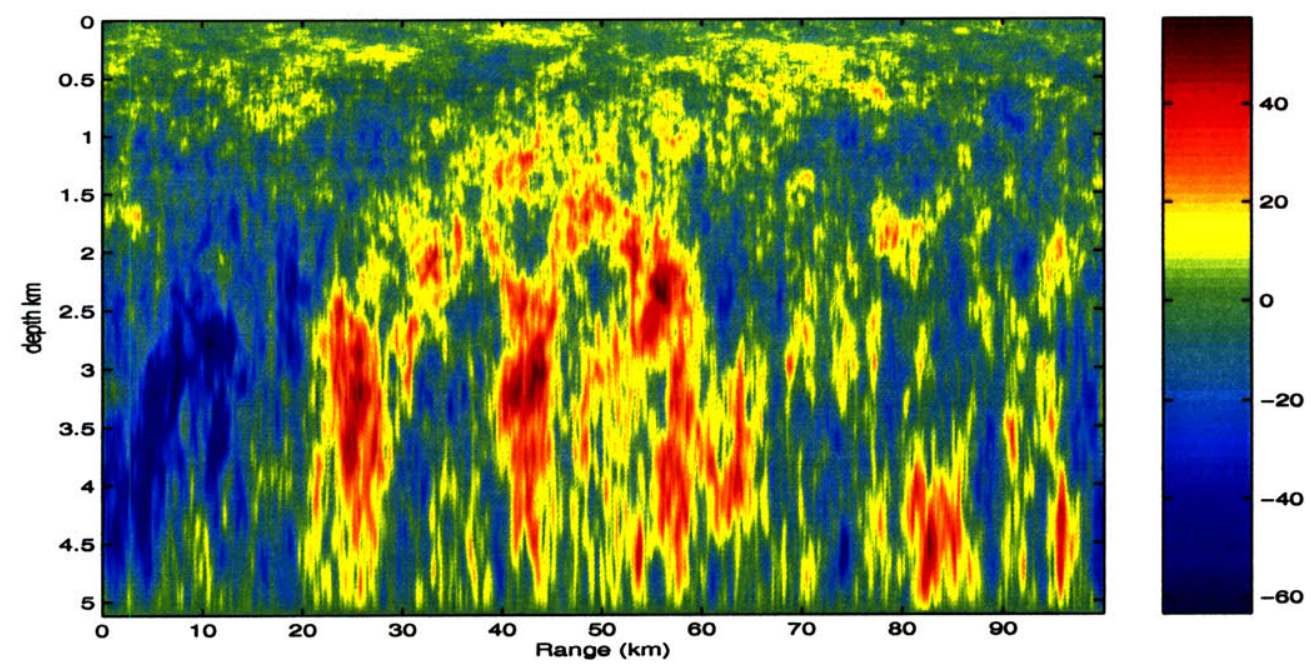

Figure 3-16: A realization of the internal-wave displacement for $\zeta_{0}=7.3 \mathrm{~m}$ and $j_{m}=100$.

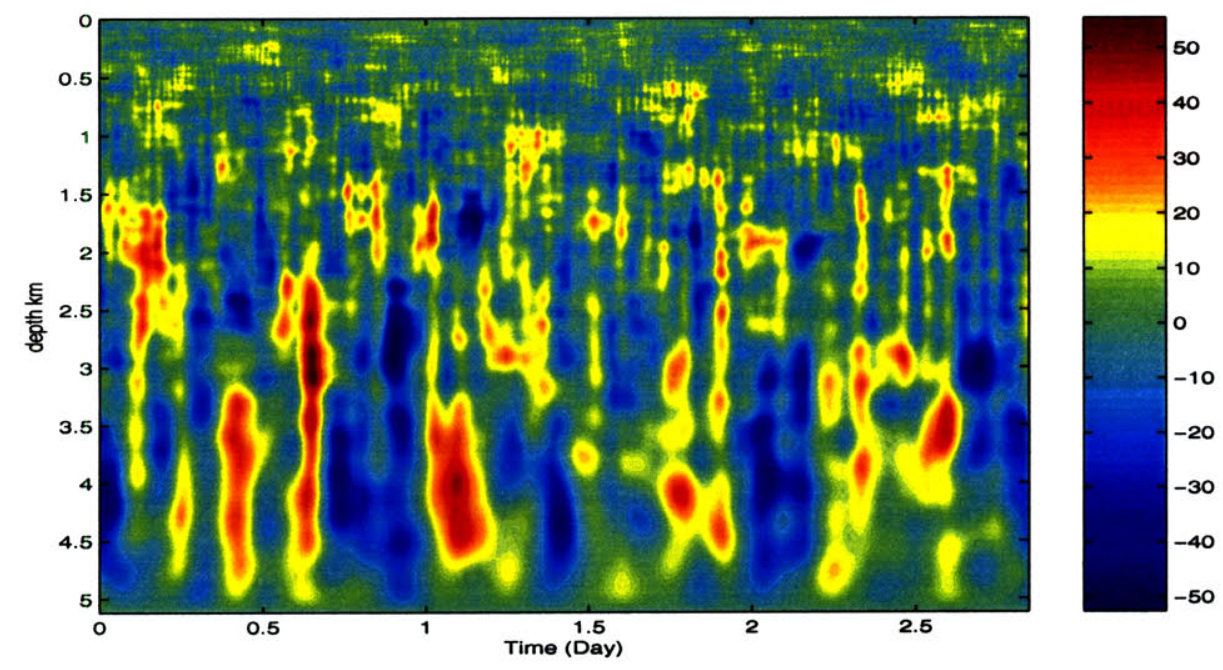

Figure 3-17: A realization of evolved internal-wave displacement field with time step of 120 seconds. 

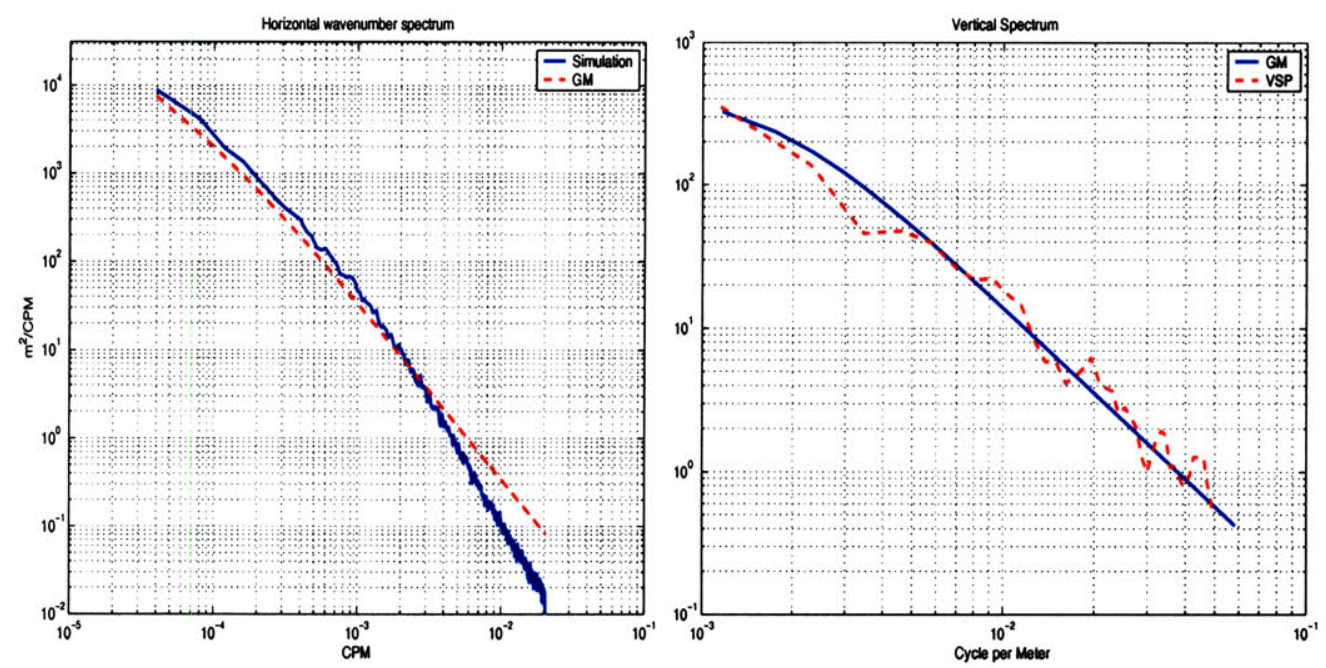

Figure 3-18: Internal wave horizontal wave number spectra (left) as modeled in this study(blue solid), and the theoretical Garrett-Munk (GM) spectrum (red dash). Internal wave vertical wave number spectra (right) as modeled in this study (blue solid), and the theoretical Garrett-Munk (GM) spectrum (red dash).

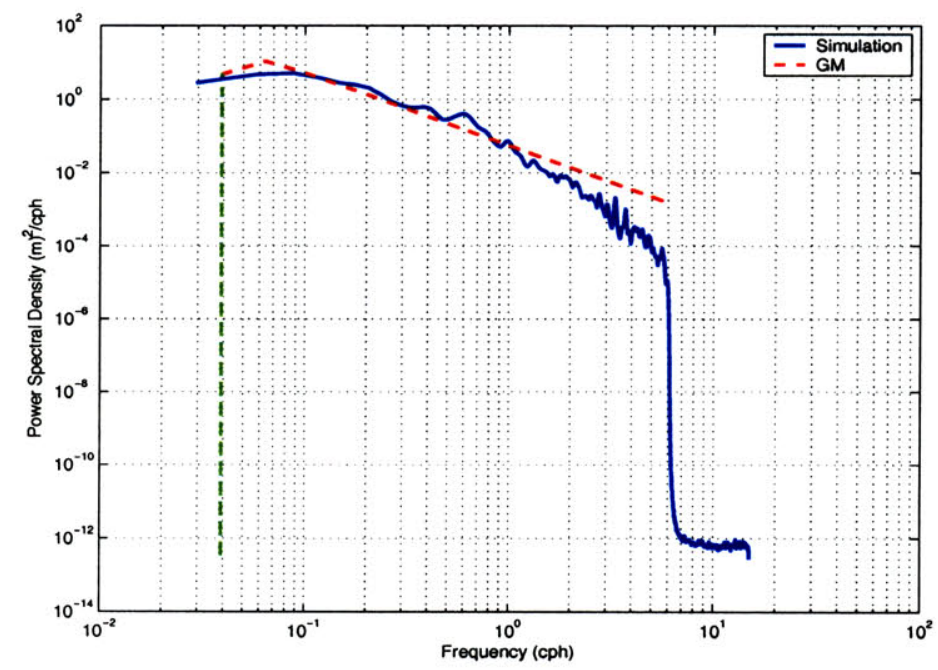

Figure 3-19: Internal wave frequency spectra as modeled in this study(blue solid), and the theoretical Garrett-Munk (GM) spectrum (red dash). Note that in this simulation, we choose $N_{0}=6.12 \mathrm{cph}$. 
Table 3.3: The source aperture and beam tilt angles(correspond to $2 \theta_{1}$ and $\theta_{2}$ in Eq. 3.33) of narrow-band beams for ID -3 and ID +4 .

\begin{tabular}{|c|c|c|}
\hline & ID -3 & ID +4 \\
\hline Source Aperture (degree) & 3.0 & 6.0 \\
\hline Beam Tilt (degree) & -10.0 & 6.5 \\
\hline
\end{tabular}

m) through this random internal wave field. In order to simulate the multipath effect of the AET experiment, two narrow beam with different beam tilt are sent out in this simulate in each time step (evolving internal wave field). So in each time step, two arrivals are obtained, which correspond to first arrival (ID -3) and second arrival (ID +4). The parameters used to specify those two narrow beam, which is addressed by Eq. 3.33, are listed in Table 3.3. Those parameters are designated based on the ray predication and configuration of vertical line array described in the previous sections.

To be consistent with the Rytov prediction, reflection from the bottom has been minimized, because we are only interested in how the sound is scattered by the water column.One example of this narrow-band beam simulation snapshot is shown in Fig. 3-20.

To compare to the Rytov prediction, the fluctuation of log-amplitude $(\chi)$ and phase $(\phi)$ must be computed. A time series of log-amplitude and phase as function of depth are obtained at each time step by normalizing the received fields with the deterministic field obtained from a non-internal wave field run. Specifically,

$$
\begin{gathered}
\chi(z, t)=\ln \left(a^{\prime}(z, t) / a_{\text {det }}^{\prime}(z)\right) \\
\phi(z, t)=\left(\phi^{\prime}(z, t)-\phi_{d e t}^{\prime}(z)\right)
\end{gathered}
$$

$a^{\prime}(z, t)$ and $\phi^{\prime}(z, t)$ are the amplitude and phase output with sound-speed fluctuations; $a_{\text {det }}^{\prime}(z)$ and $\phi_{\text {det }}^{\prime}$ are the solutions using a constant sound speed. Lastly, any $2 \pi$-discontinuities in $\phi(z, t)$ are removed.

The RMS of intensity and phase as function of depth are calculated for 2.8 days period and displayed in Fig.3-21. The average value of variance of phase and intensity for different time scale are calculated and listed in Tab. 3.4.

The Fourier spectrum analysis of these data will give the spatial vertical wave number 

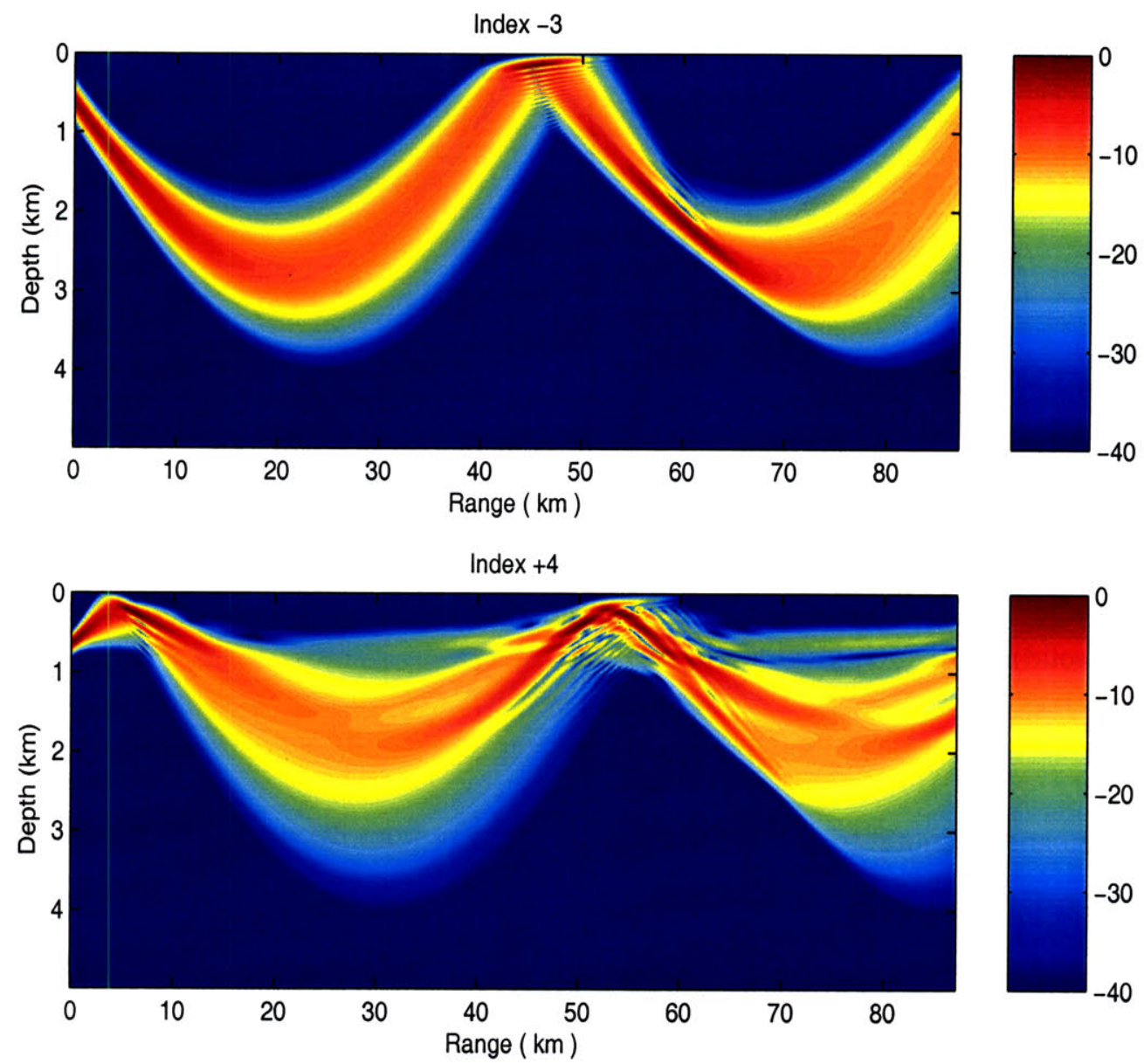

Figure 3-20: Simulation of narrow-band beam propagation. ID -3 is shown in the upper panel, ID +4 is shown in the lower panel. The colorbar is in unit of $\mathrm{dB}$ re max.
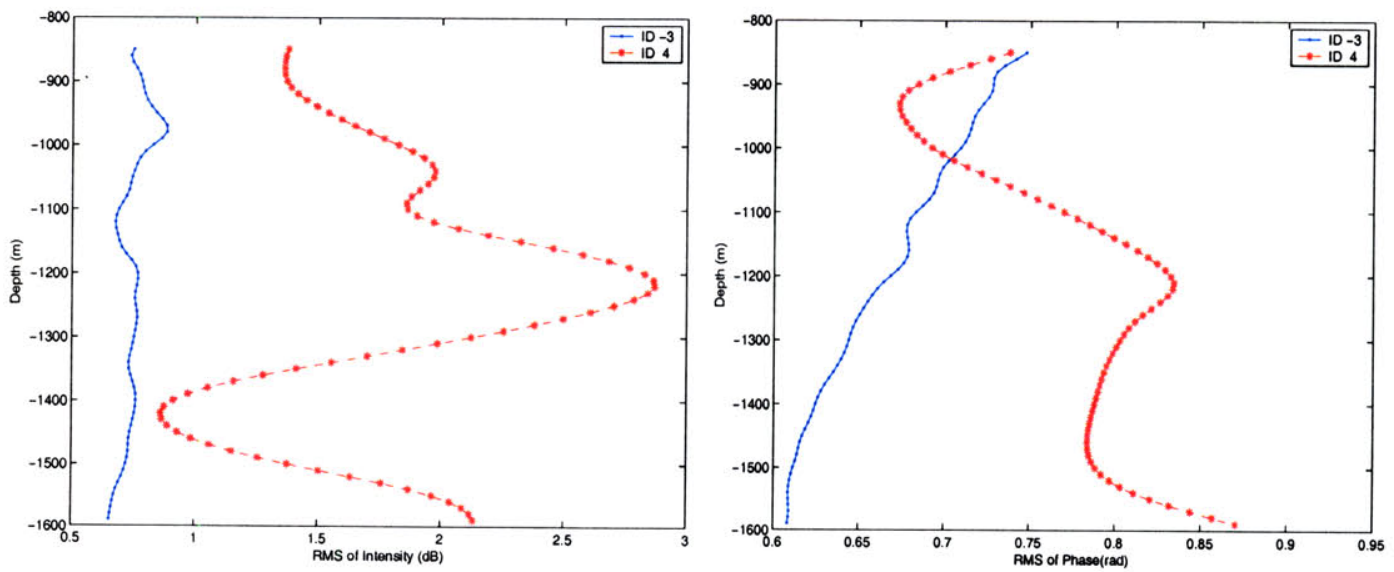

Figure 3-21: RMS of phase (right panel) and intensity (left panel) as function of depth for ID -3 (blue solid) and ID +4 (red dash) arrivals. 

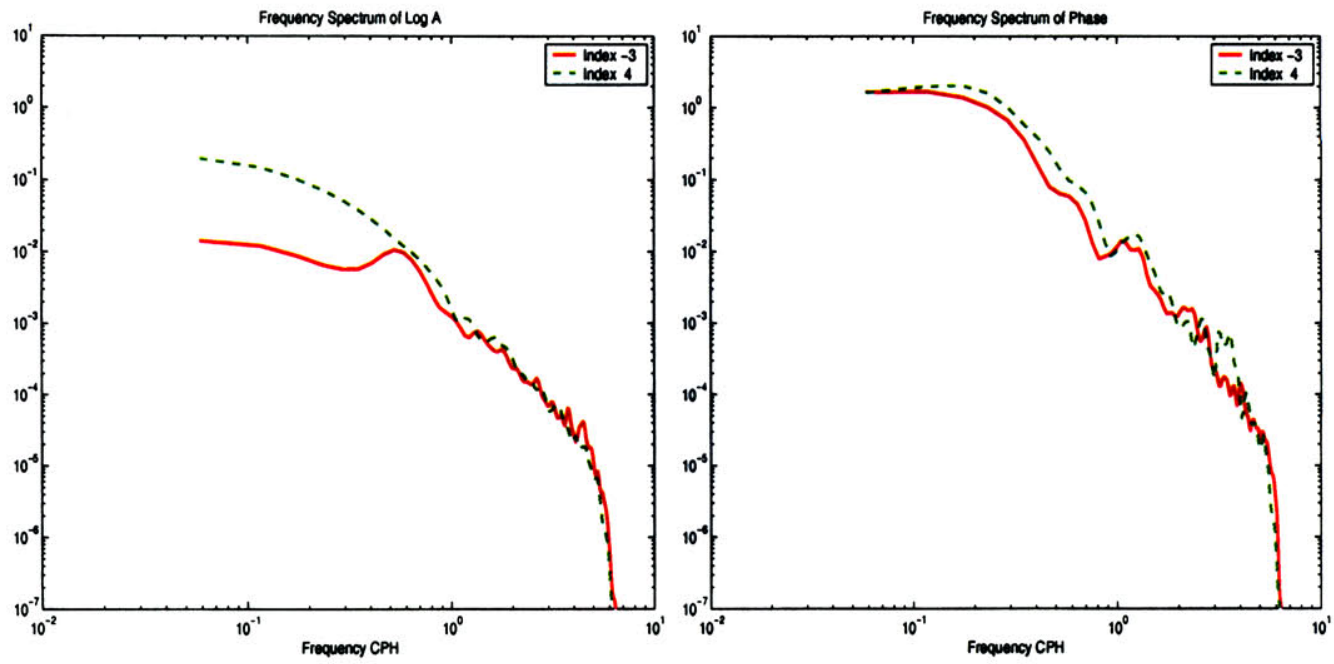

Figure 3-22: Frequency spectra of log amplitude(left panel) and phase (right panel) for ID -3 (red solid)and ID +4 (green dash) arrivals.

spectrum and temporal frequency spectrum of the wave front fluctuation to compare to the Rytov prediction from previous section.

The spectra of log-amplitude and phase of $75 \mathrm{~Hz}$ case are shown in figures Fig. 3-22 and Fig. 3-23.

In Fig. 3-22, the temporal frequency spectra are shown with log amplitude spectra in the left panel and phase spectra in the right panel. All the spectra show the cutoff at frequency of $N O=6.12 \mathrm{cph}$, which is determined by the internal wave as shown in Fig. 3-19. The frequency spectra of log amplitude in the left panel shows the feature of the depletion of low frequency variability of ID -3 relative to ID +4 , i.e. resonance condition which has been predicted in the Rytov theory. But the phase spectra do not show this feature as apparent as predicted in Fig. 3-13, which might be due to either the limitation of simulated maximum scale of internal wave, or the broadband effect on the phase fluctuation(see following broadband case).

The vertical wave number spectra are shown in Fig.3-23. All the vertical wavenumber spectra of phase and log-amplitude of ID -3 and ID +4 show the cutoff around $k_{z}=$ $10^{-2} \mathrm{cpm}$. 

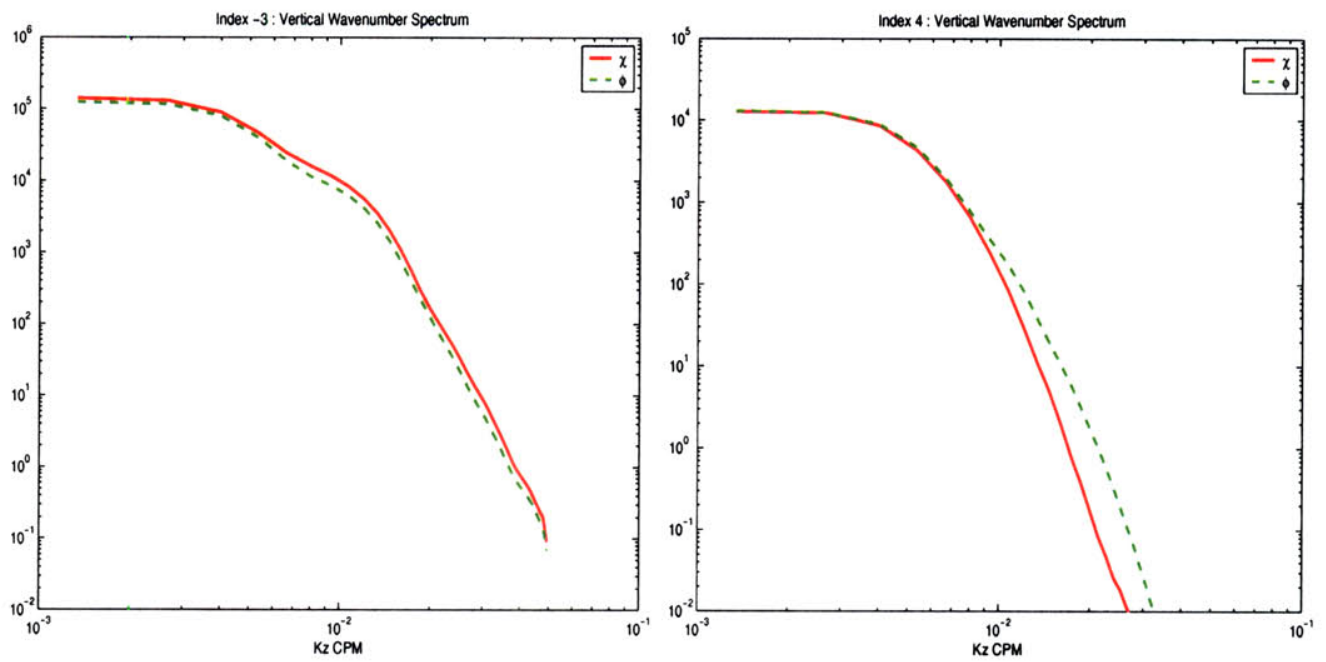

Figure 3-23: Vertical wavenumber spectra of log amplitude(red solid) and phase (green dash) for ID -3 (left panel) and ID +4 (right panel) arrivals.
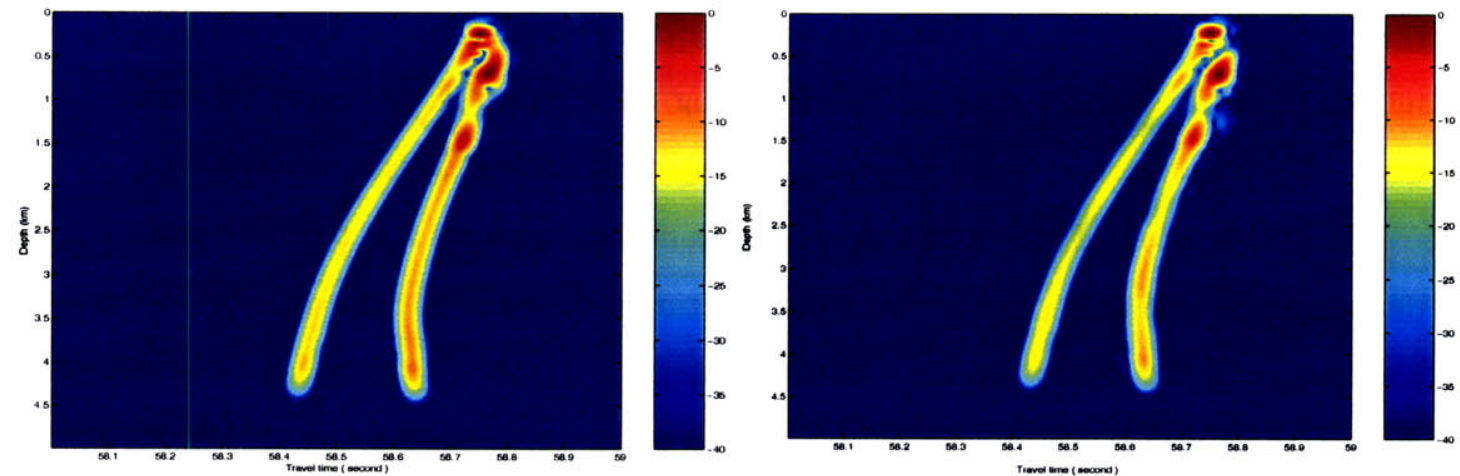

Figure 3-24: Broad-band simulation with (right panel) and without (left panel) internal wave perturbation: Wave front at $87 \mathrm{~km}$ range.

\subsubsection{Broadband Model}

The broad-band sound propagation simulation is implemented by Fourier synthesis of CW (continuous wave) results. Sixty different frequencies results, from 45 to $105 \mathrm{~Hz}$, are used. The total wave field in the time domain is achieved by Fourier synthesis for this broad-band simulation, as shown in Eq. 3.32.

The wave front at $87 \mathrm{~km}$ range are displayed without and with internal wave perturbation in Fig.3-24.

The similar procedure to Monte Carlo simulation for the narrow-band case is carried out for the broad-band case. First, a slice of internal wave field is generated at time, $t_{i}$. Then the parabolic method is used to propagate the sound wave energy through this internal 

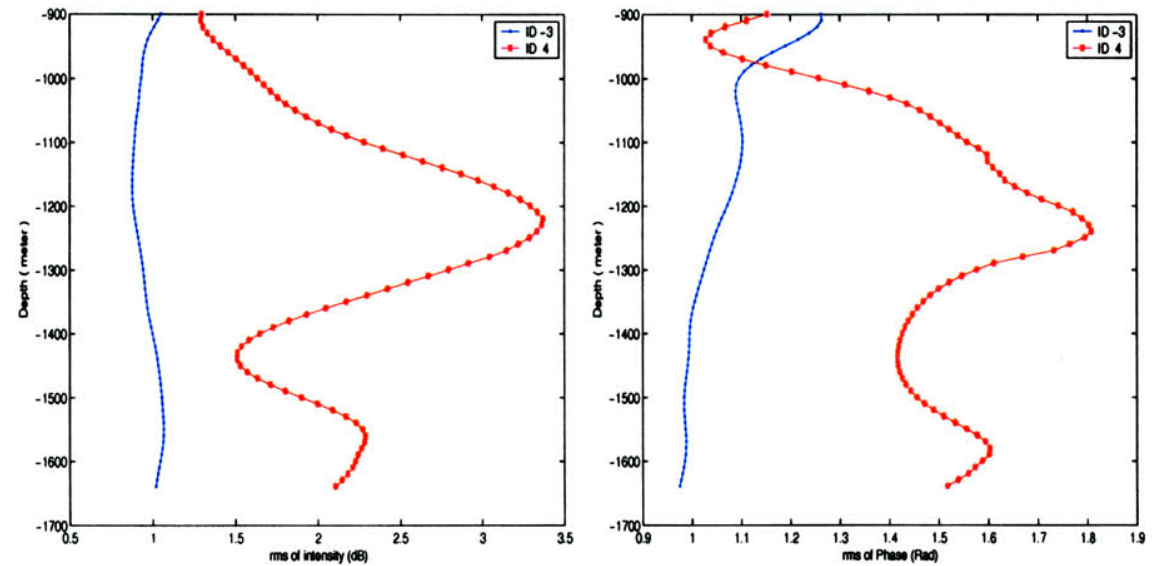

Figure 3-25: RMS of intensity (left panel) and phase (right panel) of two arrivals ID -3 (blue line with dot mark) and ID +4 (red line with star mark) as function of depth.

wave field. The sound pressure field at the received range ( $87 \mathrm{~km}$ away from source) for time $t_{i}$ is recorded. In the next step with a time interval (300 second in this case), the internal wave field will evolve in time following the internal wave dispersion relationship. Then the sound wave energy propagates through this evolved internal wave field.

The fluctuation of the sound field is obtained by normalizing received fields by the deterministic field (Fig. 3-24). To complete this broadband simulation, the two arrivals must be separated (ID -3 and ID +4), with a method explained in the AET data processing section. In general, the numerical simulation results are analyzed in a manner almost identical to the analysis of the AET experiment data.

The RMS of intensity and phase of two arrivals (ID -3 and ID +4)as a function of depth are displayed in Fig.3-25. The variance of phase and intensity as functions of depth also show the different fluctuation along the depth of two close sound wave front arrivals.

The frequency and wavenumber spectra of ID -3 wave front fluctuation (log-amplitude and phase)are shown in Fig. 3-26 and Fig. 3-27. As shown in those figures, the frequency spectra of those two arrivals have very similar structure in the spectra of high frequency (larger than $1 \mathrm{cph}$ ) region of both phase and log amplitude, which means the evolving internal wave field induces same temporal fluctuation on those arrivals in the high frequency region. In the low frequency region, both the log-amplitude and phase spectra of ID +4 have much more energy than those first arrival ID -3, which again is showing the resonance effect of internal wave and acoustic rays. Furthermore, in the phase spectra, the resonance condition is much more apparent than in the narrow-band simulation, which means the 

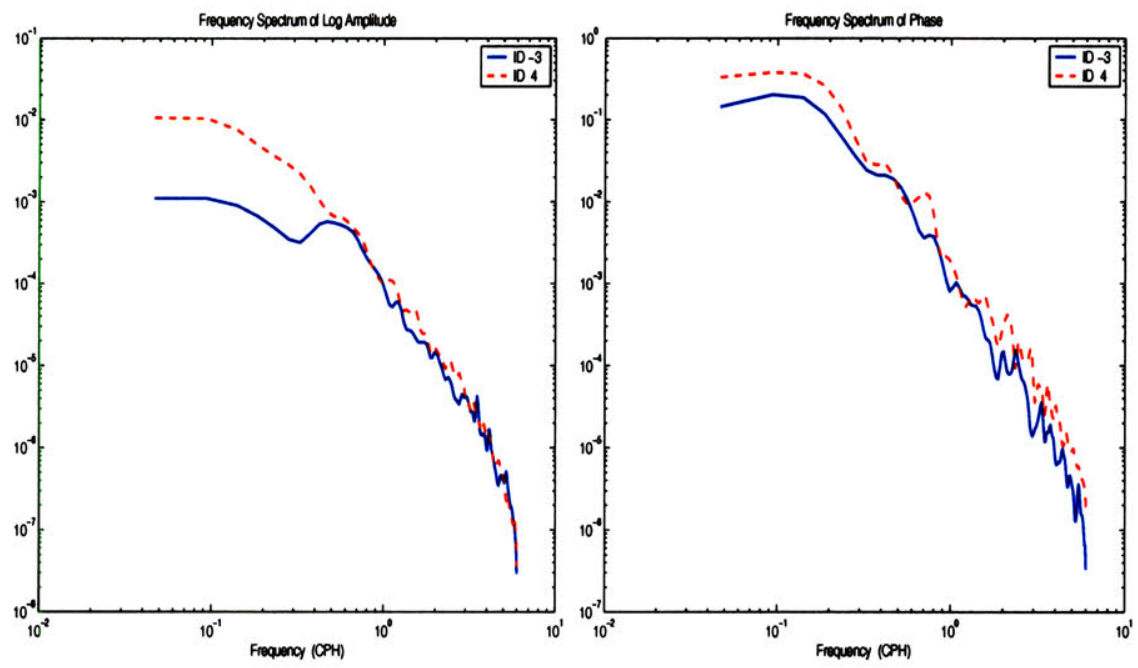

Figure 3-26: Frequency spectra of log amplitude(left panel) and phase (right panel) for ID -3 (blue solid)and ID +4(red dash) arrivals' wavefront fluctuation.

bandwidth might be critical factor for the phase behavior. All the spectra show a Rytovlike result with minus 3 slope, though two arrivals have some different behavior in the vertical wavenumber spectra.

\subsection{Data-Model Comparisons}

\subsubsection{Moments}

In Tab. 3.4, the moments of phase and intensity, which are predicted by Rytov theory, numerical simulations (narrow-band and broad-band), and observed (AET) are listed along with observation result. We found that it is a little overestimated for ID -3 , while a little underestimated for ID +4 arrival. But in general, the observation and Rytov prediction are in good agreement. In the short period observations, we found the observations and numerical simulations are in general agreement. The numerical simulations display less fluctuation in the phase, but stronger fluctuation in the intensity. The longer period simulation also shows the weak phase and intensity fluctuations, and larger value than those of short period simulations. Two continuous arrivals (ID -3 and ID +4 ) show very similar value in the short period of both experimental observations and numerical simulations. In the longer period, the second arrival (ID +4) shows stronger fluctuation in both phase and intensity than those of first arrival (ID -3), though the analysis shows that both arrival are 

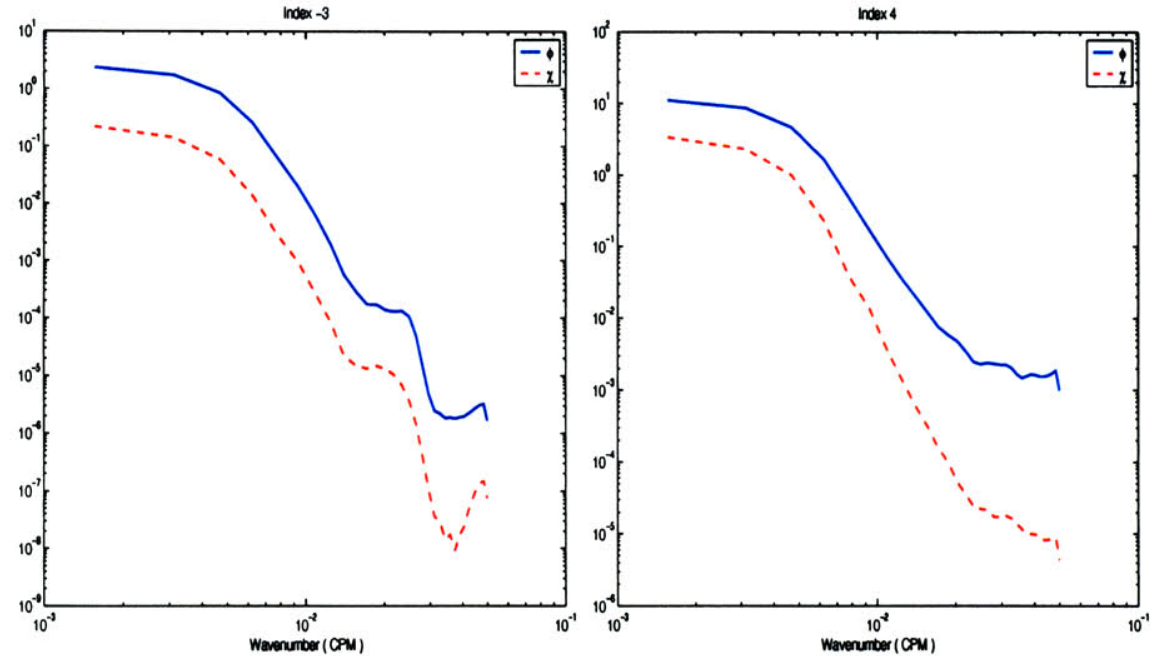

Figure 3-27: Vertical wavenumber spectra of log amplitude (red dash) and phase (blue solid) for ID -3 (left panel)and ID +4 (right panel) arrivals' wave front fluctuation.

all in the weak fluctuation region. The broad-band simulation shows much better resonance effect of internal wave and acoustic ray in the phase than the narrow-band simulation. Furthermore, the phase result of the broad-band simulation is closer to the observation result than the narrow-band simulation, which implies that the broad band is important for the phase behavior of the acoustic wave.

\subsubsection{Frequency Spectra}

In Fig. 3-28, we compare the frequency spectra of phase and log-amplitude between AET data and Rytov prediction. This figure shows the frequency spectra of log-amplitude and phase overlap with the Rytov prediction result. The lower frequency spectra are obtained by using Fourier spectral analysis of 4-hour interpolation of original AET intensity data. So the comparison is limited to only relatively high and low frequencies because of the sparse time sampling of the AET. However Figure 3-28 shows that the agreement between model and observations is satisfactory. This comparison explains the rather curious observational result that the wavefront ID -3 , which traverses the sound channel at a higher angle has much less low frequency variability than the ID +4 which has smaller angles but does not get as close to the surface. This attenuation of low frequency variability for steep rays is a consequence of the resonance condition between the local ray tilt and the internal waves with wave numbers perpendicular to this tilting ray. We believe that this result is the first 
Table 3.4: Comparison Between AET Experiment and Numerical Simulation

\begin{tabular}{|c|c|c|c|c|c|c|c|}
\hline & & \multicolumn{3}{|c|}{ ID -3 } & \multicolumn{3}{|c|}{ ID +4} \\
\hline & & $\begin{array}{c}\text { RMS of } \phi \\
(\mathrm{rad})\end{array}$ & $\begin{array}{l}\text { RMS of I } \\
(\mathrm{dB})\end{array}$ & SI & $\begin{array}{l}\text { RMS of } \phi \\
\text { (rad) }\end{array}$ & $\begin{array}{l}\text { RMS of I } \\
\text { (dB) }\end{array}$ & SI \\
\hline AET & 20-Min & $0.44 \pm 0.004$ & $0.26_{ \pm 0.013}$ & $0.0045_{ \pm 0.0005}$ & $0.44 \pm 0.002$ & $0.26 \pm 0.016$ & $0.0053 \pm 0.0007$ \\
\hline Bbsim & & $0.18 \pm 0.001$ & $0.34_{ \pm 0.001}$ & $0.0047 \pm 0.00003$ & $0.25_{ \pm 0.003}$ & $0.44 \pm 0.01$ & $0.008 \pm 0.0004$ \\
\hline NbSim & & $0.11_{ \pm 0.0004}$ & $0.29_{ \pm 0.001}$ & $0.004 \pm 0.00004$ & $0.13 \pm 0.002$ & $0.34 \pm 0.01$ & $0.006 \pm 0.0004$ \\
\hline$\overline{\mathrm{AET}}$ & 40-Min & $\overline{0.61_{ \pm 0.01}}$ & $\overline{0.38 \pm 0.02}$ & $0.0089_{ \pm 0.001}$ & $0.68 \pm 0.005$ & $0.40_{ \pm 0.054}$ & $0.013 \pm 0.004$ \\
\hline BbSim & & $0.30_{ \pm 0.001}$ & $0.51_{ \pm 0.004}$ & $0.012_{ \pm 0.0002}$ & $0.40_{ \pm 0.005}$ & $0.67 \pm 0.02$ & $0.021_{ \pm 0.001}$ \\
\hline NbSim & & $0.18 \pm 0.0009$ & $0.42_{ \pm 0.002}$ & $0.009_{ \pm 0.00008}$ & $0.23_{ \pm 0.003}$ & $0.57 \pm 0.02$ & $0.017_{ \pm 0.001}$ \\
\hline AET & 6 day & & $0.797 \pm 0.07$ & $0.044_{ \pm 0.013}$ & & $\overline{3.06 \pm 0.2}$ & $0.44 \pm 0.05$ \\
\hline BbSim & 1.8 day & $1.05_{ \pm 0.009}$ & $0.97 \pm 0.007$ & $0.051_{ \pm 0.0008}$ & $1.48_{ \pm 0.02}$ & $2.19_{ \pm 0.07}$ & $0.23_{ \pm 0.013}$ \\
\hline NbSim & 2.8 day & $0.66_{ \pm 0.005}$ & $0.75_{ \pm 0.006}$ & $0.03 \pm 0.0004$ & $0.77_{ \pm 0.006}$ & $1.79_{ \pm 0.06}$ & $0.15_{ \pm 0.01}$ \\
\hline Rytov & & 0.73 & 0.79 & 0.034 & $\overline{1.49}$ & $\overline{1.99}$ & $\overline{0.21}$ \\
\hline
\end{tabular}

observational evidence for this resonance condition.

For the phase spectra, because the longest observation period is 40 minutes, there is very short overlapped frequency band to internal wave band be used to make comparison. But the AET observation and theory are still in good agreement in general.

Figure 3-29 shows the comparison between Monte Carlo parabolic equation simulations through random fields of Garrett-Munk internal waves, and the theory for parameters close to that of the AET. The model is seen to closely predict the spectra even when a broadband signal is used(The Munk and Zacharisen Rytov theory only assumes a narrowband case).

\subsubsection{Vertical Wavenumber Spectra}

The vertical wavenumber spectra of log-amplitude and phase are compared between the observation and theory in Fig.3-30. The observation result are denoted with the marker lines, the theory result are denoted with solid lines. In general, the observation and theory are in good agreement. In the left panel, in the vertical wavenumber spectra of the logamplitude, there is roll off around $10^{-2.8} \mathrm{cpm}$. In the right panel, the phase spectra doesn't have this feature. And in the high wavenumber region of $10^{-1.9} \mathrm{cpm}$, there are roll off in all those spectra. In similar ways as the previous comparison of spectra in frequency domain, we compare the Monte Carlo parabolic equation simulations with Rytov predictions for parameters close to that of the AET experiment in the Figure 3-31. It shows that the agreement between model and observations is satisfactory in general. 

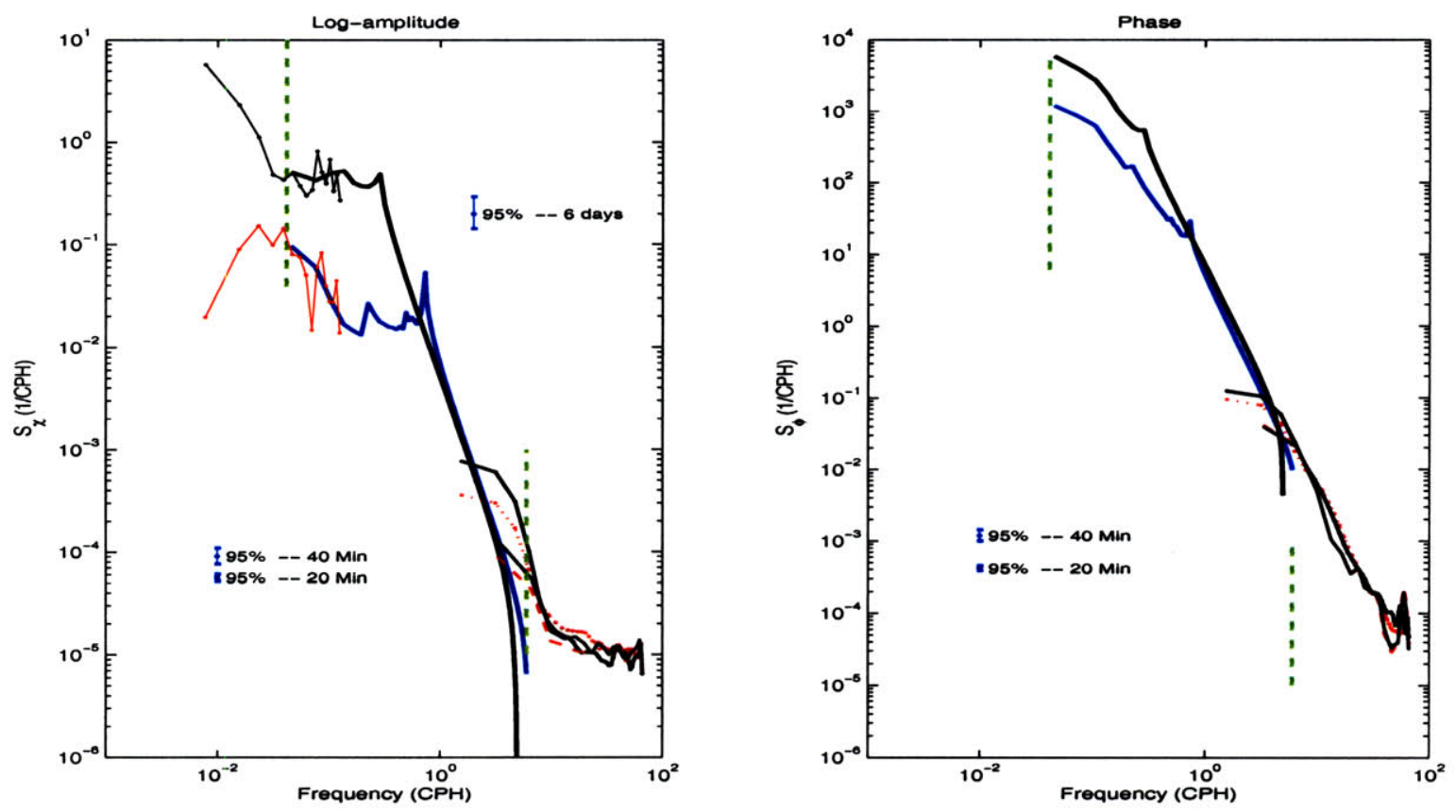

Figure 3-28: Frequency spectra of log-amplitude(left panel) and phase (right panel). The line with markers are results from observation with black for ID +4 and red for ID -3. The solid lines are theory prediction result with black for ID +4 and blue for ID -3 . The green straight vertical line denotes local inertial frequency and the local buoyancy frequency around depth $160 \mathrm{~m}$, which is $6 \mathrm{cph}$. In left panel, there are 20-min,, 40-min and 6-day observation results; in right panel, there are only 20-min and 40-min observation results for the phase. The gap in the observed log-amplitude spectra(left panel) is due to irregular sampling. 

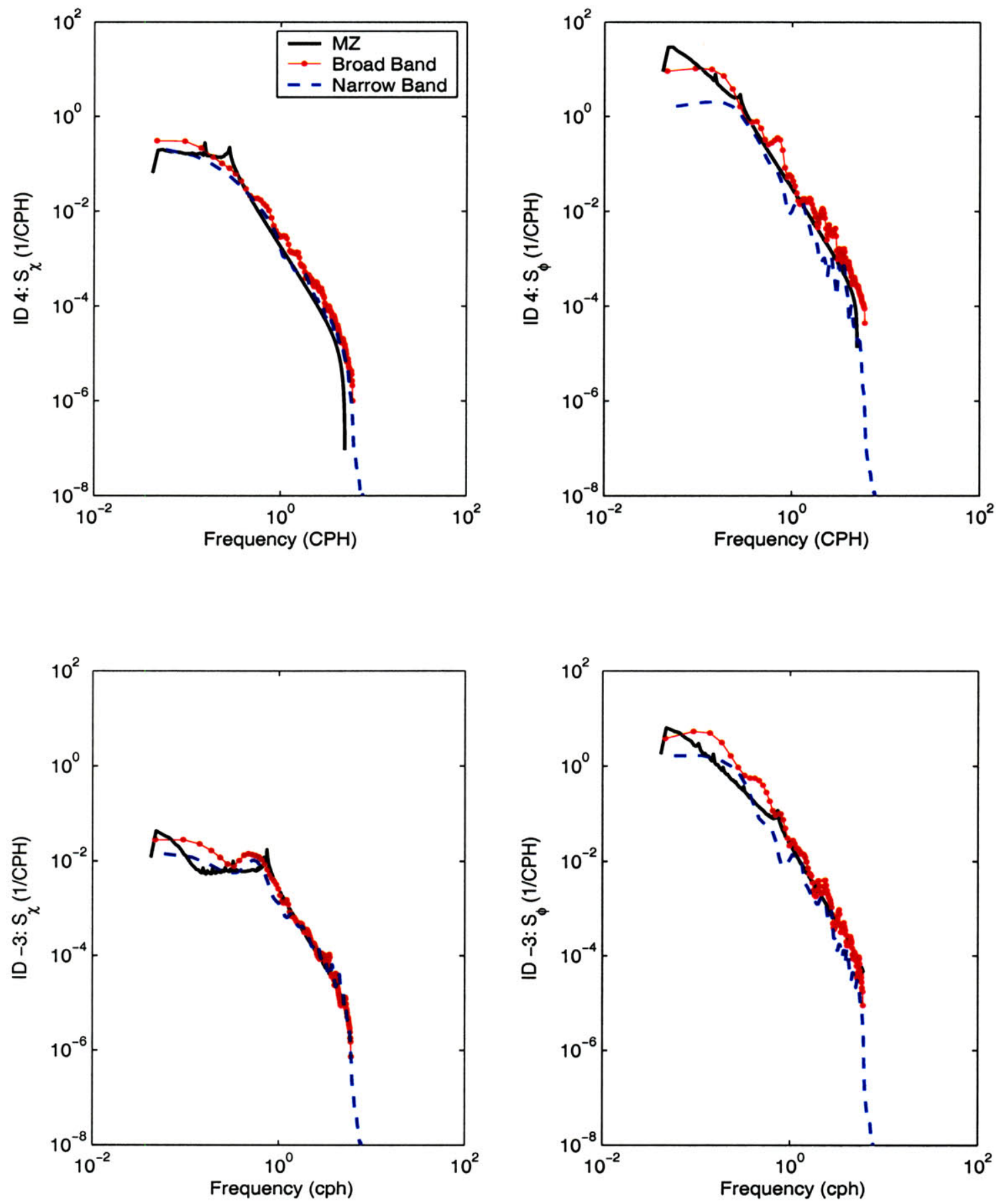

Figure 3-29: Frequency spectra of phase(right panels) and log-amplitude(left panels) from broadband(red dot), and narrow-band(dash) parabolic equation simulations of sound transmission to 87-km range through random filds of Garrett-Munk internal waves. Black curves show the Munk and Zachariasen(Rytov) predictions. Two arrivals are modeled: one has a wavefront identifier of -3 , and the other has a value of +4 . 

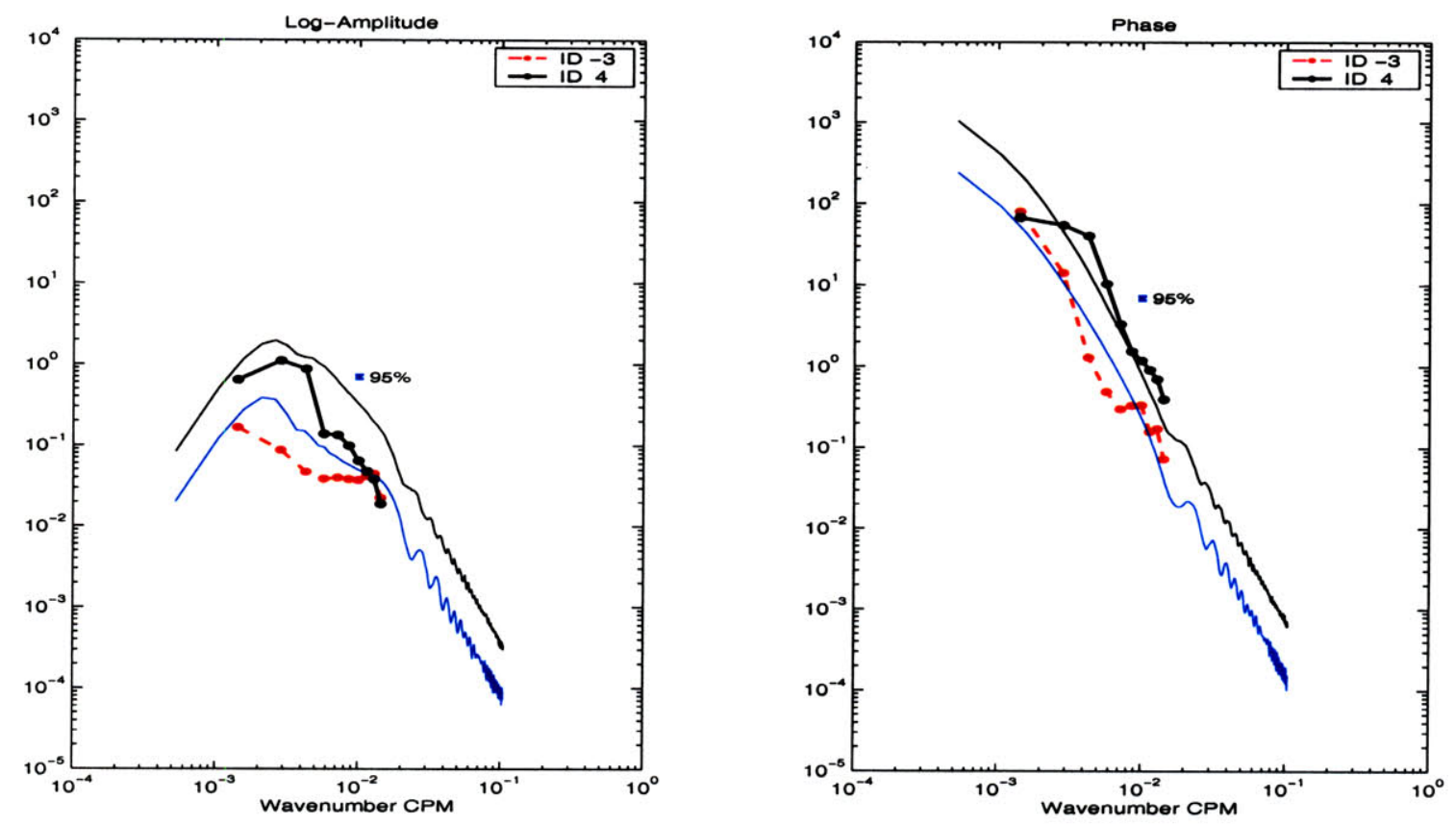

Figure 3-30: The vertical wavenumber spectra of log-amplitude (left panel) and phase (right panel). The line with markers are results from observation with black for ID +4 and red for ID -3 . The solid lines are theory prediction result with black for ID +4 and blue for ID -3 .

\subsubsection{Summary}

In this section, the procedure and method of implementing the Monte Carlo simulation of sound wave propagating through one or two upper turning points in the ocean are presented.

Two different simulations: narrow-band point source with waveguide case and broadband with waveguide, are carried out. The comparison among the numerical simulation, Rytov prediction and observation shows good agreement in moments of phase and intensity. We found the narrowband numerical simulations to underestimate the phase moment of the AET observations, and they are below the Rytov prediction. The broadband simulation has better results to compare with the narrow-band simulation in the phase variance. Both the narrow-band and broad-band simulations show the Rytov ray-internal wave resonance condition in log-amplitude as theory predicted, but the broad-band simulation shows better result of this resonance effect in the phase than the narrow-band simulation. It suggests that the bandwidth might be a key fact for the phase behavior in the sound propagation.

The comparison between the AET observation and Rytov theory prediction are in very good agreement in both moments and spectra of phase and log-amplitude, which indicates 

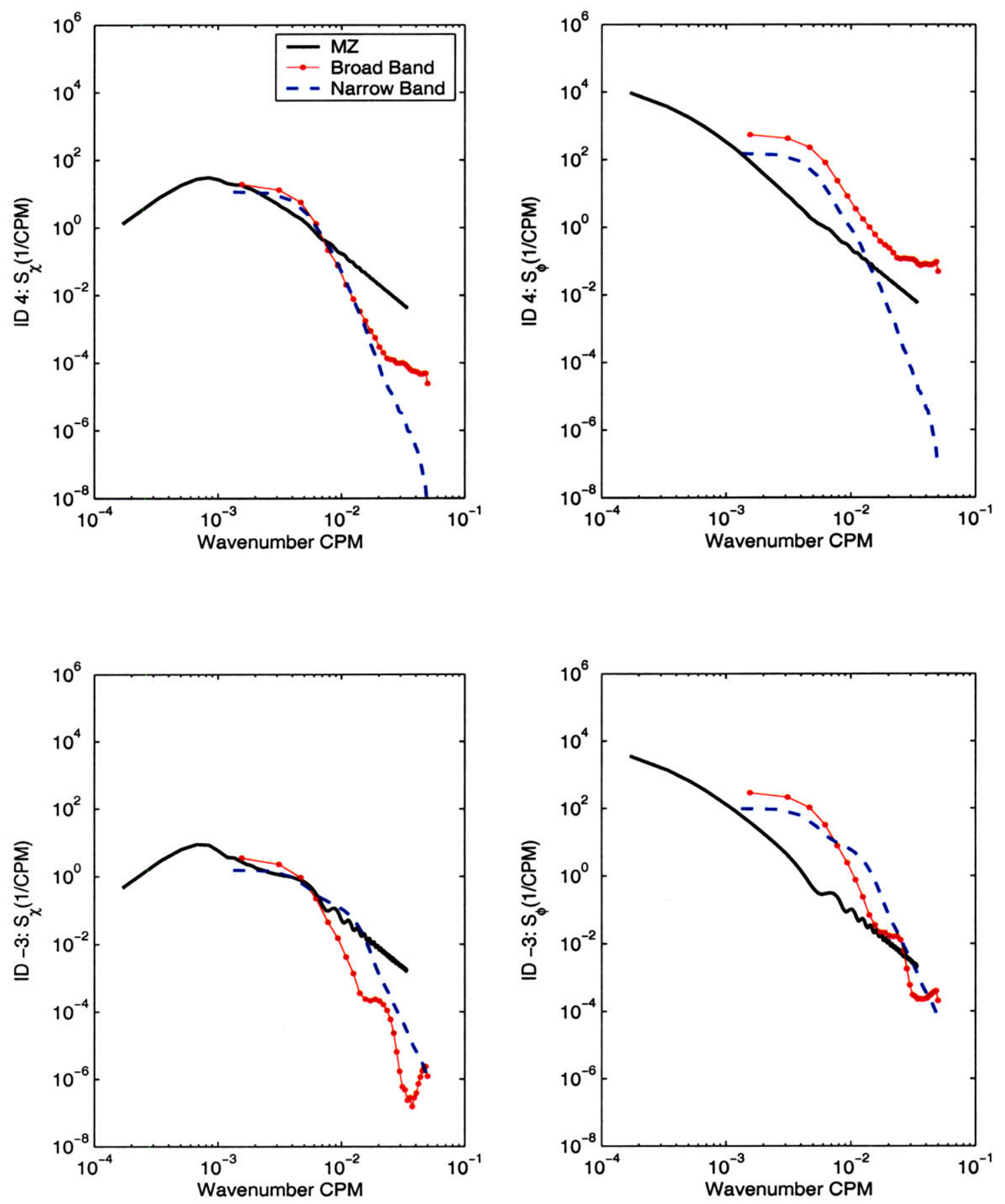

Figure 3-31: Vertical wavenumer spectra of log-amplitude (left panels) and phase (right panels) form the MZ theory, and narrowband and broadband Monte Carlo simulation. Wavefront ID's +4 and -3 are shown in the upper and lower panels respectively. 
the successful application of Rytov method in sound wave propagate in this weak fluctuation case.

\subsection{Summary}

In this chapter, an analysis of low frequency, short range acoustic transmission in the North Pacific Ocean are presented. Over a six day period, broadband signals with a center frequency of $75-\mathrm{Hz}$ were transmitted at the sound channel axis $(650-\mathrm{m}$ depth) to a $20-$ element, $700-\mathrm{km}$ long vertical receiving array located $87-\mathrm{km}$ distant, and spanning the depth region 900-1600-m. The observations reveal two time resolved acoustic paths: An early arriving path with one upper turning point and two lower turning points, and a late arriving path with two upper and two lower turning points. The space time scales of acoustic variability of phase and intensity are studied on these paths using frequency and vertical wavenumber spectra. Because data sampling occurred at 20 or 40 minute intervals followed by 2-4 hour gaps, the acoustic variability is analyzed in terms of rapid sampling rate, and multiday sampling rate. The observed variability is compared with acoustic predictions based on the weak fluctuation theory of Rytov, and direct parabolic equation, Monte Carlo simulations: In both models the source of acoustic variability is the random ocean internalwave field. The data/model comparisons suggest weak fluctuation theory may in fact be appropriate to describe the frequency and wavenumber spectra of phase and intensity for the two observed paths. Importantly the comparisons suggest that a resonance condition can exist between the local acoustic ray and internal wave field such that internal waves whose crests are parallel to the local ray path will contribute most strongly to acoustic scattering. This effect leads to an important filtering of the acoustic spectra relative to the internal wave spectra, such that rays of high incident angles do not acquire scattering contributions due to low frequency internal waves. 


\section{Chapter 4}

\section{The Range Evolution of the Mean Intensity of the LOAPEX Off-axis Source Transmissions}

The main objective of LOAPEX was to obtain observations of the range evolution of acoustic scattering, and simultaneously obtaining detailed sound speed environmental data. For LOAPEX the main focus was on acoustic observables associated with the broadband wavefront arrival pattern. For the off-axis source, ocean sound speed fluctuations can cause an in-filling of acoustic energy into the finale region. The simplest acoustic observable associated with these effects is the mean intensity, a second moment. The main efforts of this chapter are to answer the following questions: 1. How does high angle acoustic energy from an off-axis source transfer energy to low angles in the axial region of the waveguide? 2. What are the relative contributions from diffraction and scattering? 3 . How does this energy transfer scale with range?

\subsection{Introduction}

The LOAPEX cruise was coordinated with two other experiments, BASSEX, and SPICEX. The SPICE04 deployment cruise was conducted between 26 May and 18 June 2004 aboard the R/V Revelle. During this cruise two autonomous vertical line array receivers (VLAS), and two $250-\mathrm{Hz}$ acoustic transceiver moorings (500 km and $1000 \mathrm{~km}$ from VLAs) were 
deployed (Worcester, 2004). These four moorings were in place until the summer of 2005. The primary purpose of the transmissions between the $250-\mathrm{Hz}$ sources, transceivers, and the VLAs was an attempt to measure the "spiciness" of the ocean by acoustic methods. In addition to receiving the transmissions from the $250-\mathrm{Hz}$ sources, the VLAs were programmed to receive transmissions from the NPAL fixed bottom-mounted acoustic source near Kauai, $\mathrm{HI}$, and a similar acoustic source suspended from the R/V Melville during the LOAPEX cruise. The hydrophone arrays on the two combined VLAs covered most of the 5-km water column. We refer to one the VLAs as the deep VLA (DVLA), located at $33.418920^{\circ} \mathrm{N}$ latitude and $137.682470^{\circ} \mathrm{W}$ longitude. The DVLA combines a 40-element, 1400-m long array (2150-3550 m nominal ) with a 20-element, 700-m long array (3570 - $4270 \mathrm{~m}$ nominal) to span the lower caustics in the acoustic arrival pattern with a nominal spacing of $35 \mathrm{~m}$. The DVLA was considered the primary receiving array for LOAPEX. The other moored array, the shallow VLA (SVLA), was moored $3 \mathrm{n}$ mi due west of the DVLA. The SVLA has a 40-element, 1400-m long array (350-1750 m) centered approximately on the sound channel axis. Both hydrophone arrays were navigated using a network of surveyed bottom transponders.

The LOAPEX cruise was conducted aboard the R/V Melville from 10 September to 10 October 2004. The scientific objectives of LOAPEX are outlined in the following subsection.

\subsubsection{Science Objectives of LOAPEX experiment}

An acoustic signal arriving at a hydrophone array from a large distance is spread out in space and time. In mid-latitudes, the early part of the arrival is associated with steeper arrival angles and is often considered "ray-like" in that the arrivals are well characterized by frequency-independent numerical ray-tracing codes. The middle part of the acoustic arrival pattern is better characterized by acoustic modes ("mode-like"), where the final part of the arrival has highly scattered energy and is not well modeled by deterministic methods. In general, the objective is to study the evolution, with range, of the acoustic arrival pattern. The ultimate objective is to understand the range and frequency dependence of the spatial and temporal coherence, and reveal ways of improving the coherence. 


\subsubsection{Approach}

The approach to meeting the scientific objectives of LOAPEX is illustrated in Figure 4-1. The figure and its legend describe and locate the primary assets of the experiment and show the eight stations occupied by the Melville during the cruise. The eight stations are shown as red dots and seven of them are on the main LOAPEX path indicated by the solid black line. These seven stations were nominally 50, 250, 500, 1000, 1600, 2300, and 3200 $\mathrm{km}$ from the VLAs (yellow dot). These distances provided the controlled range dependency sought in this experiment. At each of these seven stations the LOAPEX acoustic source was suspended from the ship for several hours, typically one to two days. Two source depths were used at each of the seven stations, 350 and 500 ; or $800 \mathrm{~m}$. An eighth station near Kauai was also taken. This final station provides a comparison of transmissions from 300,500 , and $800 \mathrm{~m}$ depth, while the source is far from the bottom, with transmissions from the bottommounted Kauai source. Figure 4-1 also illustrates the paths from the LOAPEX stations, and from the Kauai bottom-mounted source location, to the permanently fixed acoustic receivers. These paths, along with the paths from 250-Hz SPICE04 acoustic sources, allow us to produce a "snapshot" of the Northeast Pacific Ocean's heat content.

This thesis will focus on the analysis of the transmissions for the off-axis source location (nominally $350-\mathrm{m}$ depth), and the acoustic receptions as recorded on the $1400-\mathrm{m}$ long axial receiving array. This configuration is displayed in Figure 4-2. As we can observe, the sound channel depths are different at each station, and it tended to be deeper for further stations. The sound source depth has a great impact on the the sound energy propagation, which is illustrated in Figure 4-3, in the language of local modes functions.

Some mode functions estimated for the average sound speed profile of LOAPEX experiment are displayed in Figure 4-3. The 350-m source depth gives a maximum 75- Hz mode excitation energy at mode number 20 , and there is a $40 \mathrm{~dB}$ difference between the energies in mode number 1 and 20. Equivalently, in ray language, the source excites a minimum grazing angle ray of roughly 5 (deg.), and thus the last arrival of the wavefront should be significantly advanced from the arrival time of a zero grazing angle ray or a mode 1 . As indicated in this figure (lower panels), the eigen-mode functions have an evanescent tail function at sound source depth $(350 \mathrm{~m})$, and it is apparent that if the sound channel depth is deeper (i.e. the sound source is further away from sound channel), the lower energy will 


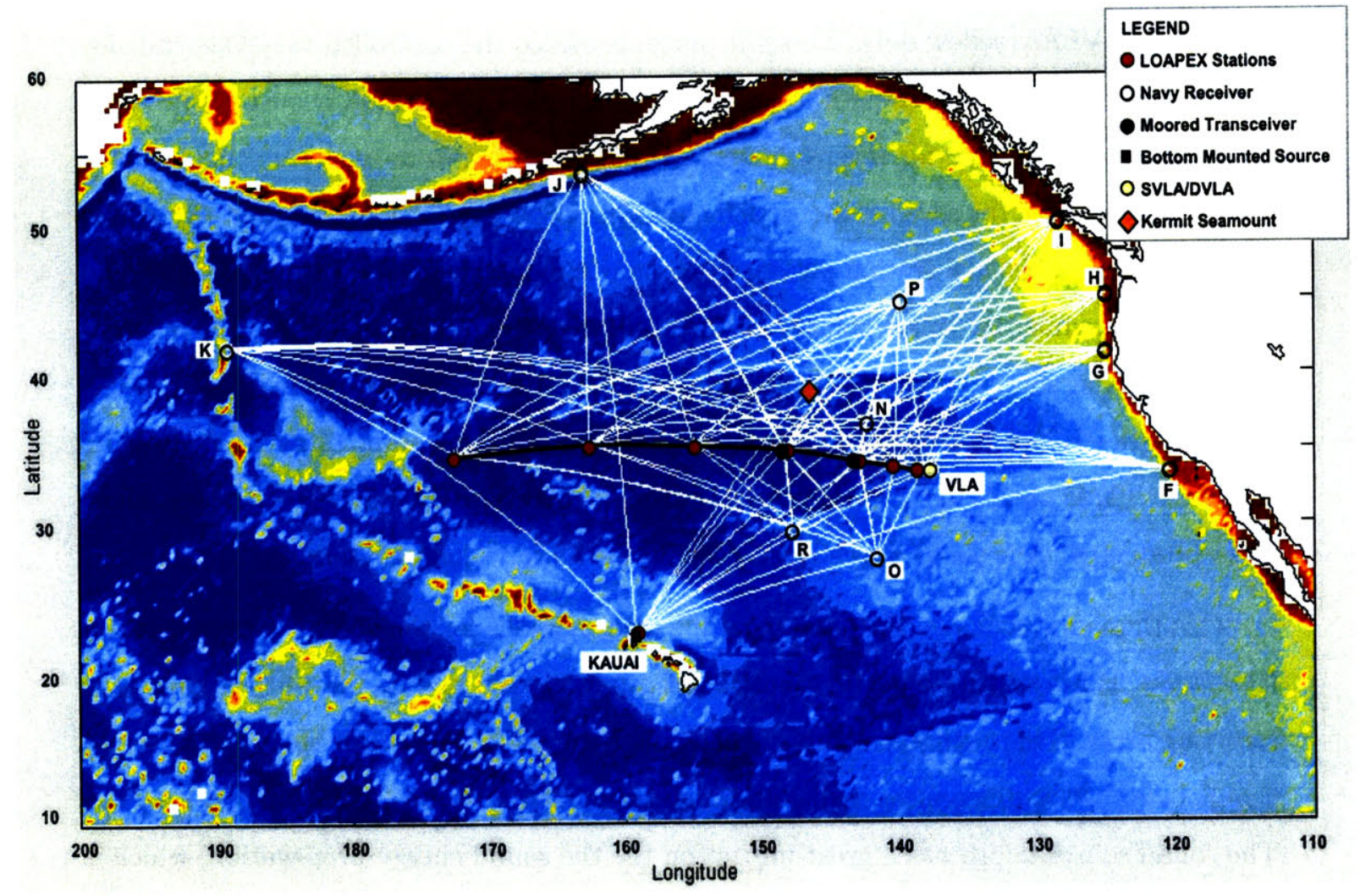

Figure 4-1: Experimental geometry. Acoustic paths from the sources[75-Hz ship-suspended LOAPEX source (red points), moored SPICE04 S1 and S2 250-Hz sources (black) 500 and $1000 \mathrm{~km}$ west of VLA, and Kauai 75-Hz source] to the receivers (S1 and S2, Navy receivers, the vertical line array, and the BASSEX towed receivers). 


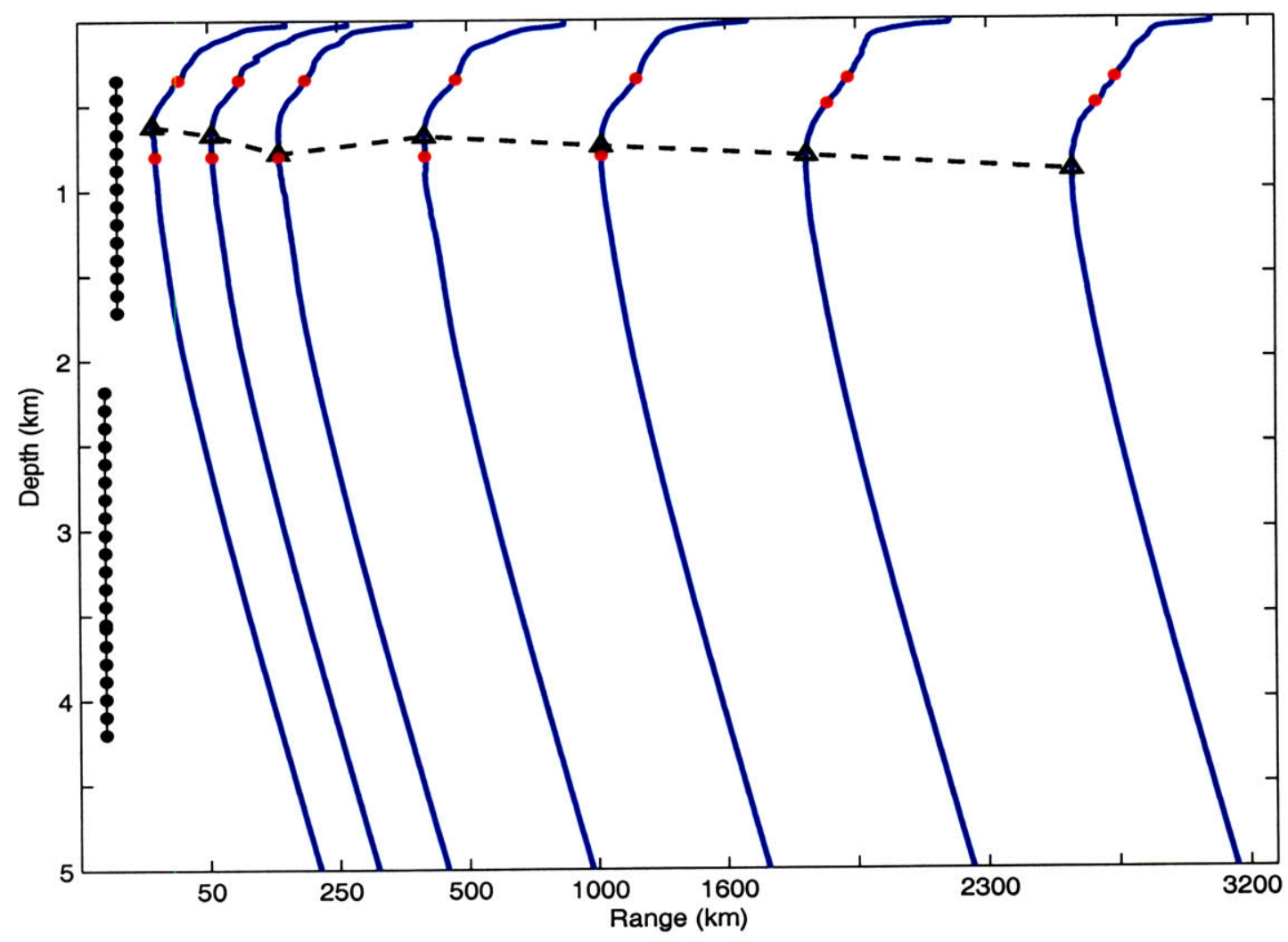

Figure 4-2: The sound speed profiles at different stations in LOAPEX experiment. The sound speed profiles are derived from the CTD measurement. The red dots on each profiles indicate the sound source depth at each station. The black triangles indicate the sound channel depth at each station. The round-dot-marks on the left indicate the shallow and deep VLA. The sound channel depth is range dependent as being shown here. 

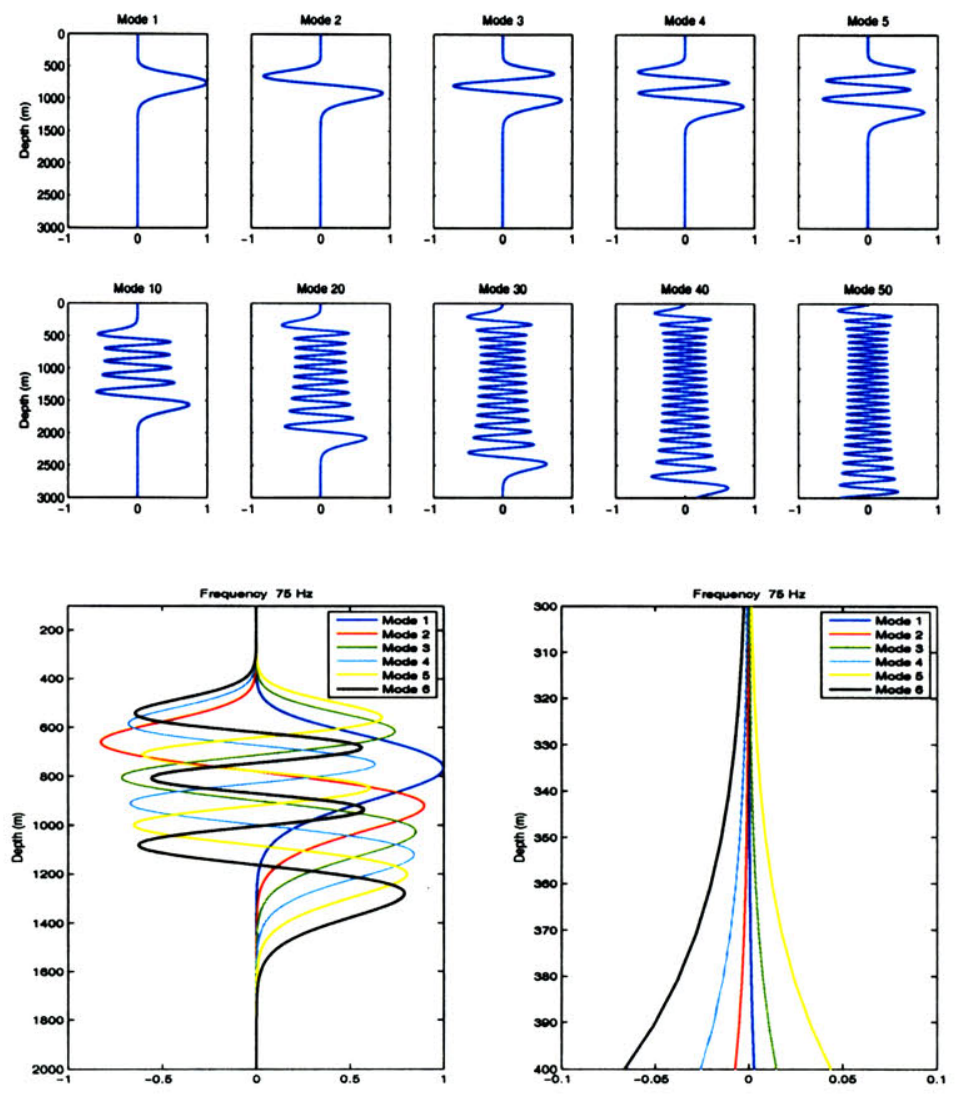

Figure 4-3: Modes functions estimated for the LOAPEX experiment. The average sound speed profile is used here. The selected number of modes are displayed in the upper panel. The lower panel shows modes 1 to 6 , with a zoom in display in the lower right panel for the depth range from $300 \mathrm{~m}$ to $400 \mathrm{~m}$.

be excited at that sound source depth for the lower order modes, which correspond to lower angle arrivals at sound channel region.

As primary acoustic observable, the mean intensity of the wavefront and its time extension relative to the arrival time of the minimum grazing angle ray will be quantified. Two processes contribute to the extension of the mean wavefront 1) diffraction from the source depth being in the evanescent region of the low order modes, and 2) acoustic scattering which can transfer energy from higher modes into the low order modes or equivalently high grazing angles into low grazing angles. In general, the diffraction and scattering could be results of two types of inhomogeneities in the ocean, regular and random. For the regular inhomogeneity such as deterministic, depth-dependent, background variation in sound speed - the ocean sound channel, the diffraction effect is well known and easy to model 

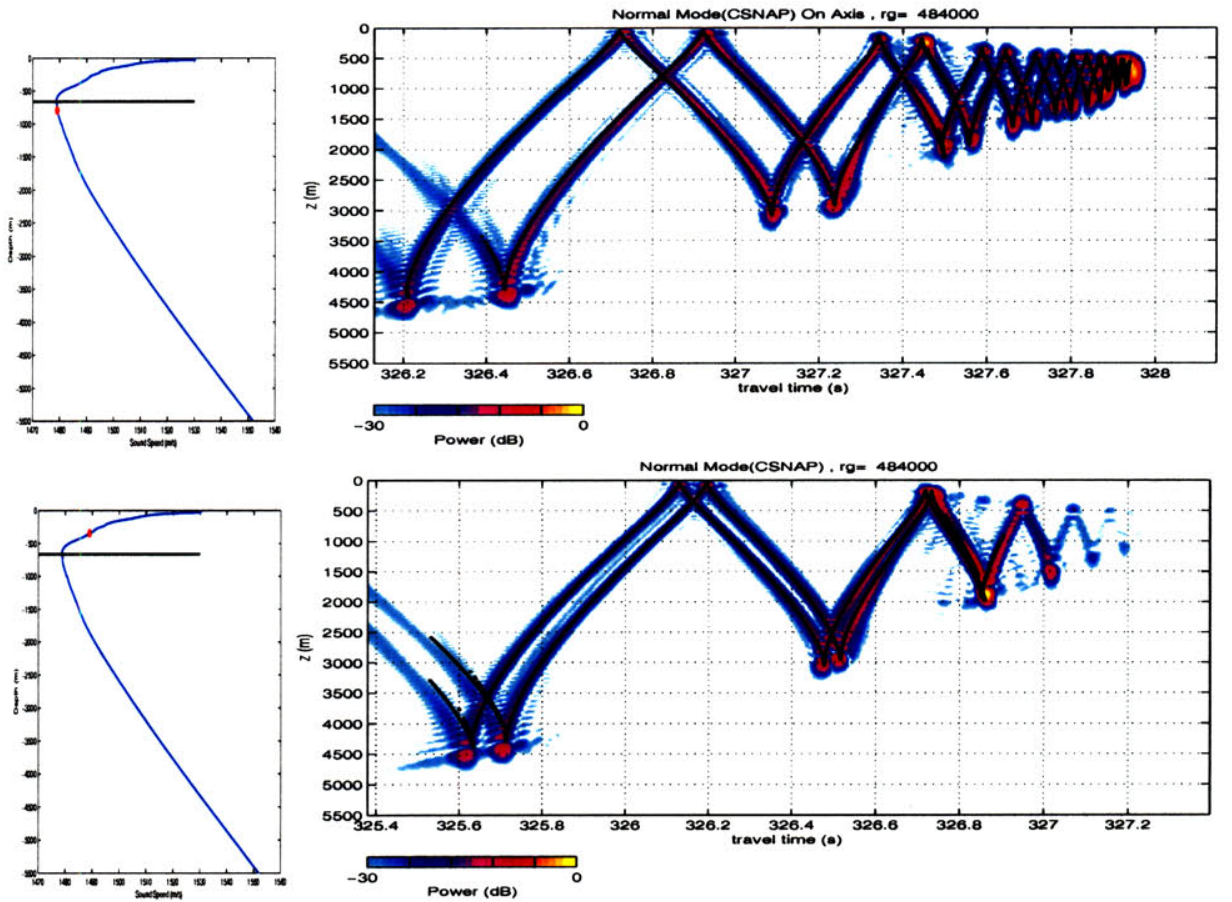

Figure 4-4: The wavefront at a range of $484 \mathrm{~km}$ broadband transmission simulations. The left two panels display the sound speed profiles with red dots indicating the sound source depth and horizontal line indicating the sound channel depth. The right upper panel shows the simulation with sound source depth of $800 \mathrm{~m}$. The right lower panel shows the simulation with sound source depth of 350 meter. In each simulation plot, there are CSNAP simulation result in color overlapped with eigenray simulations indicated by black dots.

(using deterministic mode or PE models) and leads to a wavefront time extension which scales like range. One example of different diffraction effect with different sound source depth is displayed in Figure 4-4, which show quite much acoustic intensity after last ray arrival for the case of source depth of $350 \mathrm{~m}$.

The scattering is caused by turbulence, internal waves, mesoscale eddies, etc. These random inhomogeneities cause the scattering of sound and fluctuations of its intensity, reduce coherence of sound waves and change their frequency spectrum. In general, the scattering effect is not well known, though ray and mode calculations $[19,7]$ suggest that the time extension scales like range to the $3 / 2$ power (See appendix $F$ ). Thus, at short range, the observable will be dominated by diffraction while at long range scattering should dominate. 


\subsection{LOAPEX Experiment Data Collection}

\subsubsection{Acoustic Transmission}

\section{Ship-suspended Source and Signal}

The HX-554 ship-suspended source was used during LOAPEX to transmit acoustic signals. There were seven signals used for the primary LOAPEX long-range transmissions, and two additional signals for local engineering measurements. The so called M-sequence, which is periodic repetition of a phase-coded linear maximal shift register sequence, is the signal mainly being used and analyzed in this thesis.

The signal denoted M68.2 was the full power M-sequence used at $500 \mathrm{~m}$ and $350 \mathrm{~m}$, and M75 (195) was the full power M-sequence used at $800 \mathrm{~m}$. It appeared in simulations that the best transfer of electrical power into radiated acoustic power occurred when the $M$ sequence carry frequency was about 6-8 $\mathrm{Hz}$ above the resonance frequency of the transducer. Because the transducer resonance frequency varies with depth, a carrier frequency of $75 \mathrm{~Hz}$ for $800-\mathrm{m}$ transmissions and $68.2 \mathrm{~Hz}$ for $350-\mathrm{m}$ transmissions were chosen. These depths were considered close enough that it seemed adequate to use the $68.2 \mathrm{~Hz}$ carrier signal at $500 \mathrm{~m}$, too. Simulations before the experiment suggested that it might not be possible to radiate $195.0 \mathrm{~dB}$ re: $1 \mu \mathrm{Pa} @ 1 \mathrm{~m}$ broadband from the transducer at shallow depths without exceeding the stack stress safety limit. There appeared to be no problem for the source at $800 \mathrm{~m}$ nor at $500 \mathrm{~m}$, but possibly at $350 \mathrm{~m}$. Hence, the signal designed for $350 \mathrm{~m}$ depth was scaled down so as to achieve only $194.0 \mathrm{~dB}$ re: $1 \mu \mathrm{Pa} @ 1 \mathrm{~m}$. The modulation angle is defined to be $\tan ^{2} \theta_{0}=L$, giving a smooth $\operatorname{sinc}^{2}$ envelope to the power spectrum. The other parameters are listed as in Table 4.1:

The VLA(AVATOC) receivers were programmed to sample the ship-suspended source receptions at either $300 \mathrm{~Hz}$ or $1200 \mathrm{~Hz}$, in accord with the schedule[82]. For standard transmission (44 periods): when sampling the standard transmissions at $300 \mathrm{~Hz}\left(4 f_{0}\right)$, the VLAs receive and store 40 periods (1091.2000 s, 36 periods for M68.2) of the signal, requiring $13,094,400$ bytes of buffer space in RAM, beginning at $0 \mathrm{~s}$ relative to the start of the hour. When sampling the standard transmissions at $1200 \mathrm{~Hz}$, the VLAs receive and store the signal in three groups of 11 periods each ( $300.080 \mathrm{~s}$ or 360,096 samples, 10 periods for M68.2), requiring $14,043,840$ bytes of buffer space in RAM. The recording windows start at $0 \mathrm{~s}, 400 \mathrm{~s}$ and $800 \mathrm{~s}$ relative to the start of the hour. For long transmissions (176 periods), 
Table 4.1: M-Sequence signal parameters

\begin{tabular}{c|c|c|c}
\hline & M68.2(194) & M75(195) & \\
\hline source depth & $350(500)$ & 800 & $\mathrm{~m}$ \\
\hline source level & 234 & 263 & $\mathrm{~W}$ \\
& 194 & 195 & $\mathrm{~dB}$ re $1 \mu \mathrm{Pa} @ 1 \mathrm{~m}$ \\
\hline center frequency $f_{0}$ & 68.2 & 75 & $\mathrm{~Hz}$ \\
\hline cycles per digit & 2 & 2 & $\mathrm{~ms}$ \\
\hline digit length & 29.325 & 26.6667 & digits \\
\hline sequence length L & 1023 & 1023 & $\mathrm{~s}$ \\
\hline sequence period & 30.00 & 27.28 & $\mathrm{~s}$ \\
\hline sequence law & $2033_{8}$ & $2033_{8}$ & deg \\
\hline sequence initialization & $1000_{8}$ & $1000_{8}$ & in 20 minute \\
\hline phase modulation angle & 88.209215 & 88.209215 & 44 \\
\hline sequence repetitions transmitted & 40 & 34 & for stations up to T1600 \\
\hline sequence recorded at VLA & 30 & 33 & for stations of T2300, T3200 \\
\hline sequence recorded at VLA & 36 & $/$ &
\end{tabular}

Table 4.2: LOAPEX Station coordinates, with range to the deep and shallow VLA and source depths

\begin{tabular}{c|c|c|c|c|c}
\hline Station & $\begin{array}{c}\text { Latitude N } \\
\text { (decimal deg. })\end{array}$ & $\begin{array}{c}\text { Latitude E } \\
(\text { decimal deg. })\end{array}$ & $\begin{array}{c}\text { DVLA } \\
(\mathrm{km})\end{array}$ & $\begin{array}{c}\text { SVLA } \\
(\mathrm{km})\end{array}$ & $\begin{array}{c}\text { Depth } \\
(\mathrm{m})\end{array}$ \\
\hline T50 & 33.513590 & 138.208350 & 50 & 44.7 & $350 / 800$ \\
\hline T250 & 33.869780 & 140.322990 & 250 & 244.8 & $350 / 800$ \\
\hline T500 & 34.248840 & 142.882500 & 490 & 484.7 & $350 / 800$ \\
\hline T1000 & 34.864170 & 148.280130 & 990 & 984.7 & $350 / 800$ \\
\hline T1600 & 35.285610 & 154.949970 & 1600 & 1594.7 & 350 \\
\hline T2300 & 35.312730 & 162.647970 & 2300 & 2294.7 & $350 / 500$ \\
\hline T3200 & 34.631820 & 172.472870 & 3200 & 3195 & $350 / 500$ \\
\hline
\end{tabular}


when sampling the long transmissions at $300 \mathrm{~Hz}\left(4 f_{0}\right)$, the VLAs receive and store the first hour of the signal in three groups of 40 periods each, with the recording windows starting at $0 \mathrm{~s}, 1200 \mathrm{~s}$, and $2400 \mathrm{~s}$ relative to the start of the hour. When sampling the long transmissions at $1200 \mathrm{~Hz}$, the VLAs will receive and store the first hour of the signal in nine groups of 11 periods (10 periods for M68.2)each, with the recording windows starting at $0 \mathrm{~s}, 400 \mathrm{~s}, 800 \mathrm{~s}, 1200 \mathrm{~s}, 1600 \mathrm{~s}, 2000 \mathrm{~s}, 2400 \mathrm{~s}, 2800 \mathrm{~s}$, and $3200 \mathrm{~s}$ relative to the start of the hour. In both cases the next normal recording window for standard transmissions occurring hourly will complete the recording of the long transmissions.

\section{Ambient Noise, SNR, and Receiver Gain}

At $75 \mathrm{~Hz}$, distant ship traffic is the dominant source of ambient noise. The SPICE moorings are in intensity zone IV, but close to the boundary between intensity zones IV and V, for ship-generated ambient noise. Zones IV and V have predicted spectral levels of $75.2 \mathrm{~dB}$ and $80.0 \mathrm{~dB}$ re $1 \mu \mathrm{Pa} / \sqrt{\mathrm{Hz}}$, respectively (Sadowski, Katz, and McFadden, Ambient Noise Standards for acoustic Modeling and Analysis, Naval Underwater System Center, 1984). The predicted signal-to-noise ratios (SNR) for the LOAPEX source, which has a source level of $195 \mathrm{~dB}$ (for 800/500 m) and $194 \mathrm{~dB}$ (for $350 \mathrm{~m}$ ) re $1 \mu \mathrm{Pa} @ 1 \mathrm{~m}$ (260 watts), are given in Table 4.3 for station T500,T1000 as examples.

\section{Source Motion}

Knowledge of the absolute source position is required for the tomographic application. Knowledge of the relative source motion, on time scales of $10 \mathrm{~s}$ to $80 \mathrm{~min}$ and spatial scales of $2 \mathrm{~m}$ to $5 \mathrm{~m}(1 / 10$ to $1 / 4$ of the wavelength at $75 \mathrm{~Hz})$ is required for the acoustic propagation aspects of the experiment, especially the temporal and spatial coherence estimates. Several measurement systems were used to provide data for estimating source position as a function of time:

1. C-Nav GPS measuring A-frame position where the source cable enters the water

2. Acoustic Doppler Current profiler (ADCP) measuring low-frequency currents to 800 m

3. Acoustic tracking of the source relative to a bottom transponder 
Table 4.3: Signal-to-noise ratios for the $75 \mathrm{~Hz} \mathrm{HX}-554$ transmissions at ranges of 500 and $1000 \mathrm{~km}$ with source depth of $350 \mathrm{~m}$. The SNR at a single hydrophone for a resolved ray arrival are given. The spreading loss calculations for a single ray conservatively assume pure spherical spreading in first $10 \mathrm{~km}$ then cylindrical spreading afterward. Attenuation is calculated for the North Pacific Ocean using Lovett $(A=0.055)$.

\begin{tabular}{|c|c|c|c|}
\hline & T500 & T1000 & \\
\hline Source Level (rms) & 194 & 194 & $\mathrm{~dB}$ re $1 \mu \mathrm{Pa} @ 1 \mathrm{~m}$ \\
\hline Spreading loss & -106.9 & -113.0 & $\mathrm{~dB}$ \\
\hline Volume attentuation $(0.0043 \mathrm{~dB} / \mathrm{km})$ & -0.21 & -0.42 & $\overline{\mathrm{dB}}$ \\
\hline Received signal level & $\overline{86.9}$ & $\overline{80.6}$ & $\overline{\mathrm{dB}}$ \\
\hline Noise $(1 \mathrm{~Hz}$ band $)$ & 75.2 & 75.2 & $\mathrm{~dB}$ re $1 \mu \mathrm{Pa} / \sqrt{H z}$ \\
\hline Bandwidth, $Q=2(37.5 \mathrm{~Hz})$ & 15.7 & 15.7 & $\mathrm{~dB}$ re $1 \mathrm{~Hz}$ \\
\hline Total noise level & $\overline{90.9}$ & $\overline{90.9}$ & $\mathrm{~dB}$ re $1 \mu \mathrm{Pa}$ \\
\hline Broadband SNR (before processing) & -4.0 & -10.3 & $\mathrm{~dB}$ \\
\hline Period averaging gain (10 periods) & 10.0 & 10.0 & $\overline{\mathrm{dB}}$ \\
\hline Pulse compression gain(1023 digits) & 30.1 & 30.1 & $\overline{\mathrm{dB}}$ \\
\hline Total signal processing gain & $\overline{40.1}$ & $\overline{40.1}$ & $\mathrm{~dB}$ \\
\hline Single hydrophone SNR & 36.1 & 29.8 & $\mathrm{~dB}$ \\
\hline
\end{tabular}

4. MicrocCat pressure and temperature at the source (1.5 meter suspending below), to provide source depth

5. S4 current meter to provide relative current between the source and the water

The first two, GPS and ADCP, are used as the forcing for a cable dynamics model (J. Gobat, APL-UW) to estimate source position on a second-by-second basis. The balance of the measurements are used to partly to tune the model (primarily horizontal drag coefficient for the cable) and partly to verify the model. The details of the approach taken to remove relative source motion are described in the following section.

\subsubsection{Environmental Measurements}

Observation of ocean sound speed structure were carried out using the SIO Underway CTD (UCTD) system, expendable bathyermographs (XBT)s, and the shipboard Seabird CTD. Other environmental observations included the deployment of two APL-UW Seagliders, ocean currents from the ship by 75-kHz ADCP (providing profiles from $750 \mathrm{~m}$ to $1000 \mathrm{~m}$ depth under idea conditions), and ocean bathymetry from the ship's multibeam system. 


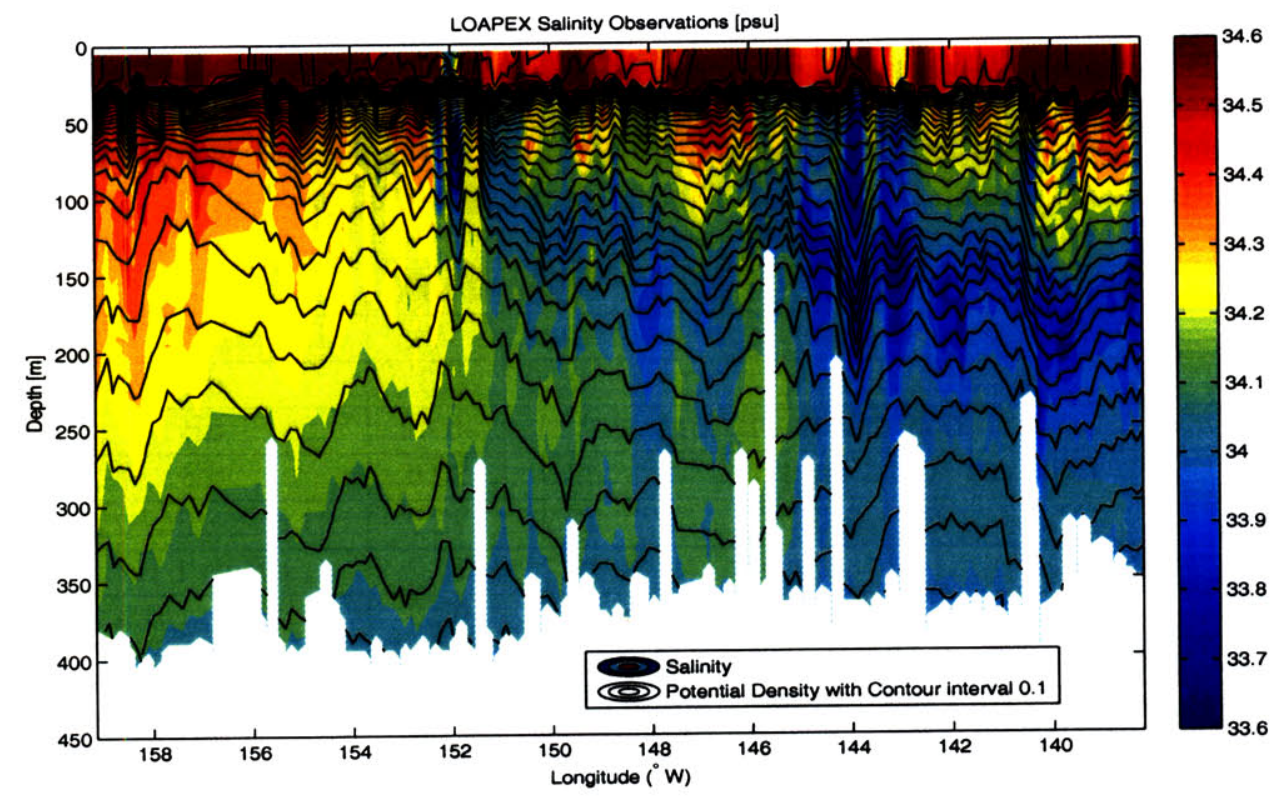

Figure 4-5: LOAPEX salinity section with potential density contours.

\section{The Underway CTD (UCTD)}

The UCTD operates under the same principle as an XBT. By spooling tether line both from the probe(with temperature, conductivity and depth sensors) and a winch aboard ship, the velocity of the line through the water is zero, line drag is negligible, and the probe can get arbitrarily deep. Measurements were made almost continually while in transit, starting at the VLA position. A total of 177 UCTD casts were carried out. The main 2000-km transect lasted 10 days and consisted of 156 casts. UCTD data were collected during the east-west transect between source locations T50, T250, T500, T1000, T1600, and T2300. Casts ranged in depth from a minimum of roughly $200 \mathrm{~m}$ to a maximum of $410 \mathrm{~m}$, and were separated in time by 30-45 min giving a nominal 10-15-km range resolution.

Figure 4-5 shows the UCTD salinity measurements with isopycnal contours. Regions where isopyncals cross lines of constant salinity are regions of intrusive fine structure (spice). The mixed layer depth varied between $20 \mathrm{~m}$ and $40 \mathrm{~m}$, with a strong gradient of density between $40 \mathrm{~m}$ and $50 \mathrm{~m}$. The salinity minimum near the base of the mixed layer, which weakens to the west. The salinity minimum near the base of the mixed layer, which weakens to the west, is a well known feature of this region. A strong frontal feature is evident around $153^{\circ} \mathrm{W}$. Weaker fronts are evident around $147^{\circ} \mathrm{W}$ and $141^{\circ} \mathrm{W}$. 


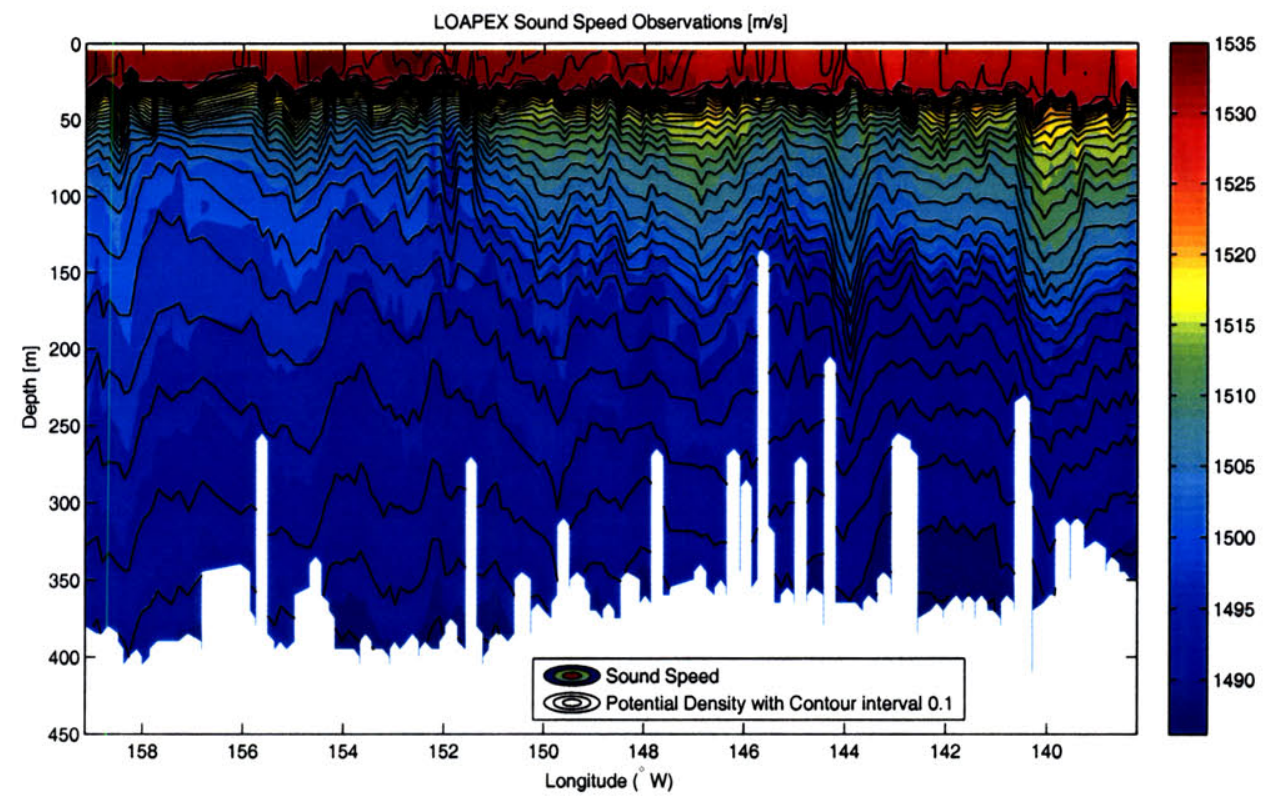

Figure 4-6: LOAPEX sound speed section with potential density contours.

Figure 4-6 shows the UCTD sound speed section, which is of fundamental interest to the LOAPEX acoustic propagation studies. As in previous figure, isopycnal contours are plotted over the section. Regions where the isopycnals cross contours of constant sound speed are where intrusive fine structure(spice) exists. At fixed depth sound speed is seen to increase from the east to the west, evidence of some range dependence in the background sound speed profile.

\section{Ocean Depth CTDs}

Full water depth CTD casts were done at stations T250, T500, T1000, T1500, T2300, T3200. Figures 4-8 and 4-7 show sound speed and buoyancy frequency derived from the seven CTD casts along the T50-T3200 section. Deep sound speeds and buoyancy frequencies are very consistent across the section. The sound channel axis is seen to deepen after passage through the front at $153^{\circ} \mathrm{W}$

\section{XBTs}

During the LOAPEX sections between T50 and T3200, 102 XBT casts were made to resolve temperature variability at horizontal resolution of 25-50 km and larger. During the UCTD 

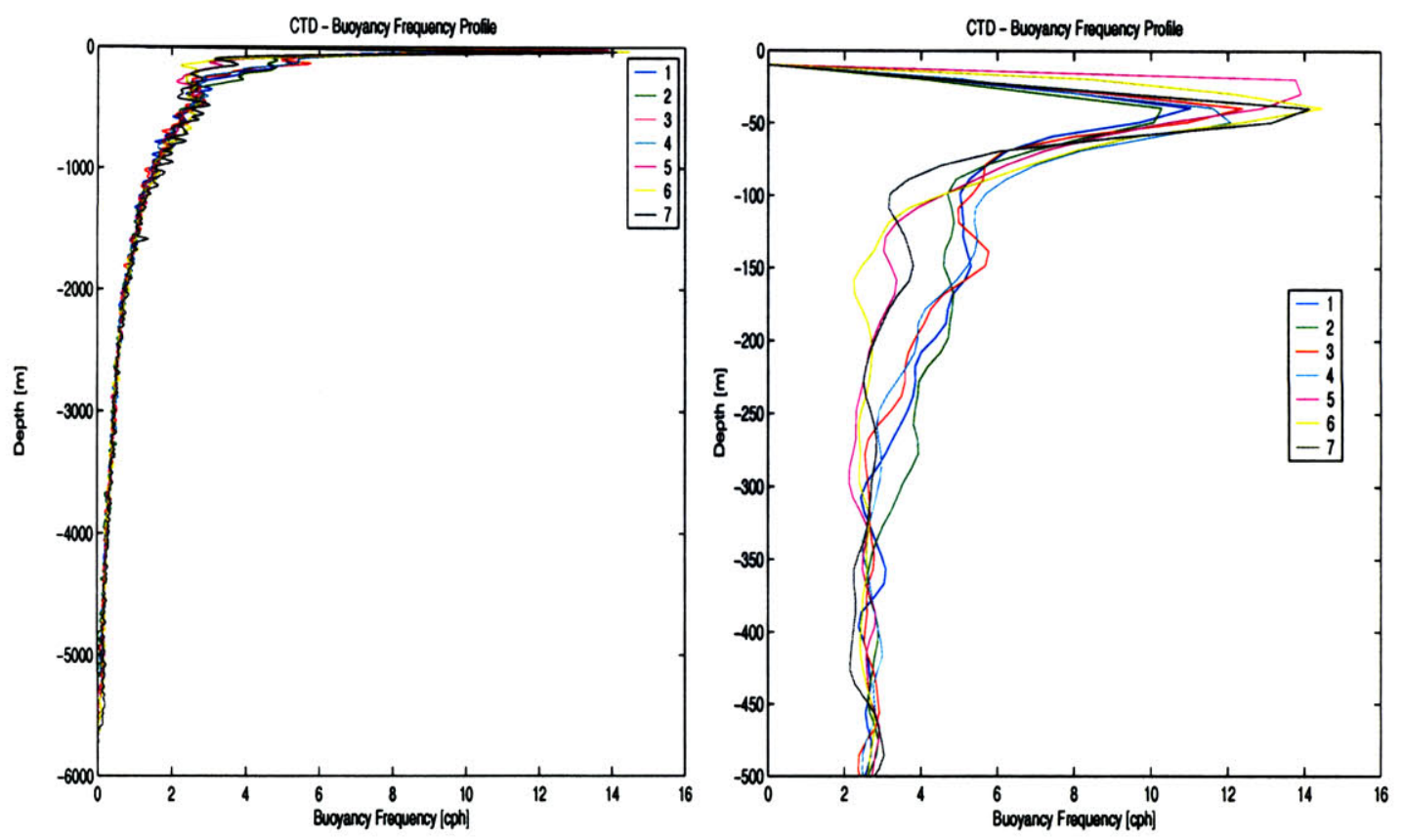

Figure 4-7: Buoyancy frequency profiles from the LOAPEX CTD section. Curves 1-7 correspond to staions T50, T250, T500,T1000, T1600, T2300, and T3200. Right panel displays upper ocean variability.
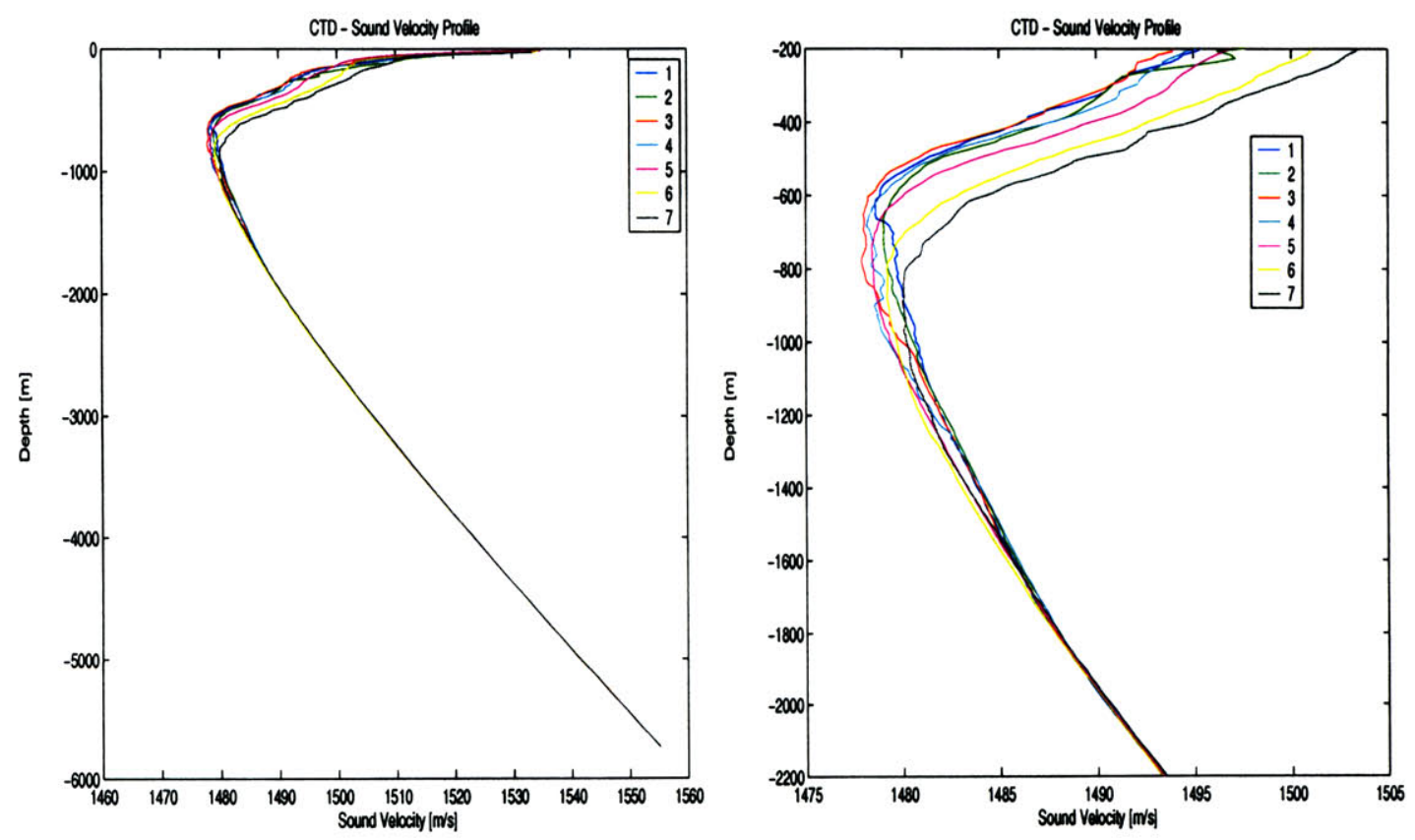

Figure 4-8: Sound speed profiles from the LOAPEX CTD section. Curves 1-7 correspond to staions T50, T250, T500,T1000, T1600, T2300, and T3200. Right panel displays upper ocean variability. 


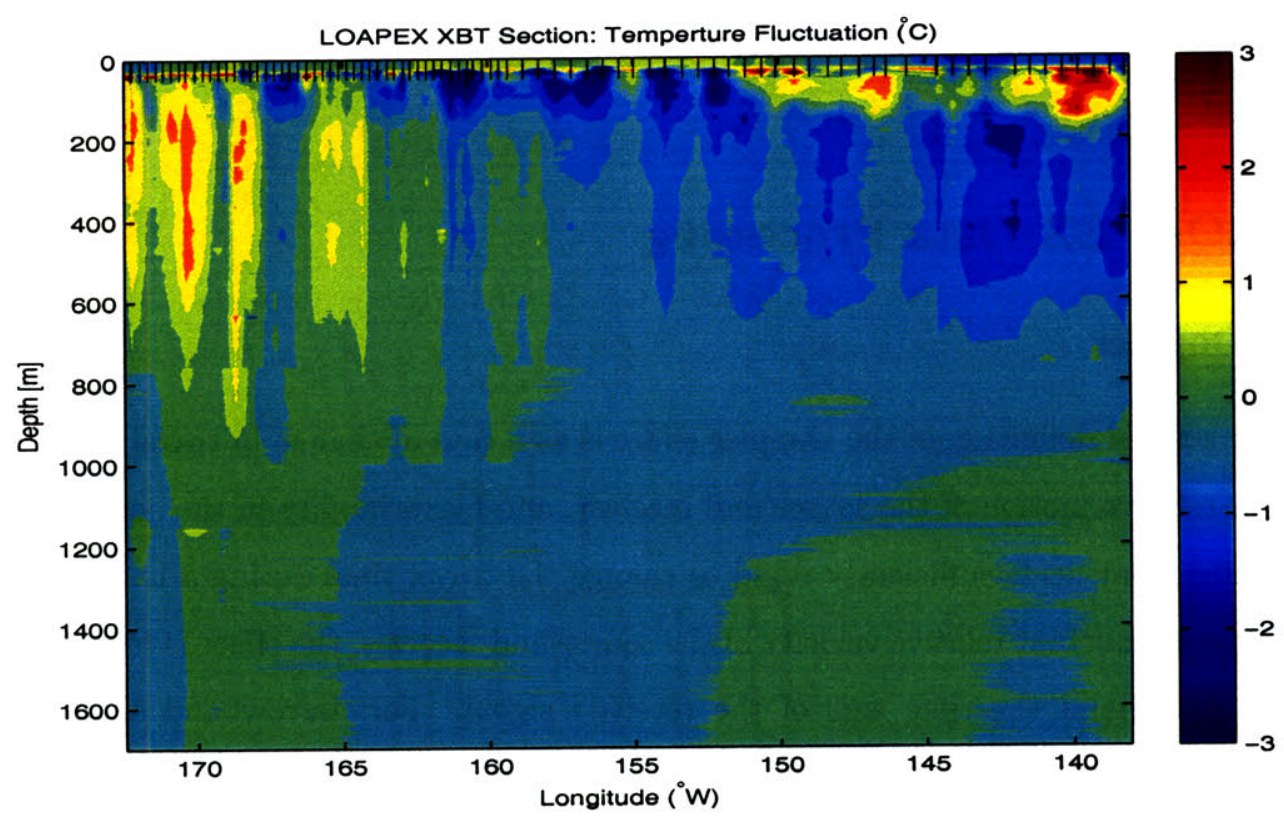

Figure 4-9: Temperature fluctuations from the LOAPEX XBT section. Black ticks in the top of the panel mark where casts were made. Probe depths varied between $760 \mathrm{~m}, 1000 \mathrm{~m}$, and $1830 \mathrm{~m}$. Much of the deep section below $760 \mathrm{~m}$ is filled in by horizontal interpolation.

operations XBTs were deployed every $50 \mathrm{~km}$, and after the suspension of UCTD at roughly $160^{\circ} \mathrm{W}$, the XBTs were dropped every $25 \mathrm{~km}$. Overall 72 T-6 (760-m depth), 12 Deep Fast (1000-m depth), and 15 T-5 (1830-m depth) XBTs were deployed. Figure 4-9 shows the observed temperature fluctuations from the XBT data. To the east there are a few strong, near-surface features $(0-200 \mathrm{~m})$, while to the west, some moderate strength, but large, vertical scale features are evident (perhaps internal tides).

\section{Environmental Mooring Data on SVLA}

A combination of Seabird SBE 37-SM MicroCAT and SBE 39-SM Temperature Recorders (MicroTemp) sensors provided point measurements of the temperature, salinity, and pressure on the VLAs for the entire year from June 2004 to June 2005. Ten of the MicroCATs have pressure sensors rated to $1000 \mathrm{~m}$; one of the MicroCATs has a pressure sensor rated to $3500 \mathrm{~m}$. The MicroCATs measure temperature, conductivity, and pressure. The MicroTemps measure temperature only. The sampling intervals were set up as follows: 7 minutes for the MicroCAT instruments and 5 minutes for the MicroTemp instruments. The majority of the instruments, 18 MicroTemps and 10 MicroCATs, were mounted on the 
SVLA, which cover the depth range from $150 \mathrm{~m}$ to $1600 \mathrm{~m}$ with average interval of 60 meter.

\subsection{LOAPEX Data Processing}

\subsubsection{Doppler}

The most general definition of the Doppler effect is as a rate of change in travel time. It is caused by relative motion of the source and receiver, and by variability in the ocean soundspeed and current fields sufficiently rapid to change the travel time during a transmission. For the case of constant relative velocity of the source and receiver, the effect is to uniformly compress or expand the time axis of the received signal. For narrowband signals, the principal result is the familiar frequency shift

$$
\frac{\Delta f}{f}=\frac{v}{C}
$$

where $v$ is the velocity at which the source and receiver are approaching. For broadband signals of the type used in ocean acoustic tomography, the envelope is also significantly compressed by a factor $(1+(v / C))^{-1}$. As we know, Doppler limits the time over which a signal can be coherently processed. For constant Doppler (e.g., constant relative velocity of the source and receiver), the solution is to process for a range of possible Dopplers and to select the output with the maximum value. One proceeds by selecting a mesh of uniformly spaced Doppler compression ratios (i.e., relative speeds). For each hypothesized speed, the data are interpolated and resamples to obtain samples at the times that would have been sampled in the absence of Doppler. This can be done directly on the complex demodulates.

For periodic signals of the type that we used in the LOAPEX experiment, the resampling must be done prior to forming the period average. Finally, resampled signal for each Doppler compression ratio is processed, and the one with the largest peak is selected.

\subsubsection{Estimation of the Transmission Loss at Receiver Distance}

\section{Sonar Equation}

The sonar equation is simply a systematic way of estimating the expected SNR at a distant receiver, taking into account the source characteristics, geometric spreading with range, 
attenuation, boundary effect, ambient noise, and the receiver characteristics. The sonar (sound navigation and ranging) equation models the expected SNR for transmission from a source to receiver:

$$
S N R=S L-T L-(N L-A G) \quad \mathrm{dB}
$$

where TL is transmission loss, NL is noise level at the receiver, and AG is (receiving) array gain, all expressed in decibels.

\section{Transmission loss}

Transmission loss includes attenuation and geometric spreading, $T L=T L_{a}+T L_{g}$, in decibels. Attenuation is linearly proportional to range, $T L_{a}=\alpha r$. Francois and Garrison (1982a,b) and Garrison et al. (1983) provide comprehensive summaries of what is known about sound absorption in the ocean. An approximate expression for the attenuation coefficient $\alpha$, valid for low frequencies(below about $8 \mathrm{kHz}$ ) and at the depth of the sound-channel axis is

$$
\alpha(f)=0.79 A \frac{f^{2}}{(0.8)^{2}+f^{2}}+\frac{36 f^{2}}{5000+f^{2}} \quad \mathrm{~dB} / \mathrm{km},
$$

where $f$ is in kilohertz (Fisher and Simmons, 1977; Lovett, 1980). The first term is due to boric acid relaxation, which depends on ocean $\mathrm{pH}$ through the coefficient $\mathrm{A}$, and the second term is due to magnesium sulfate relaxation, which is independent of $\mathrm{pH}$. At frequencies below $1 \mathrm{kHz}$, the first term is dominant. Lovett (1980) provides charts of the coefficient A for the Atlantic, Indian, and Pacific oceans. It varies by a factor 2 between the North Pacific $(A=0.055)$ and the North Atlantic $(A=0.11)$.

The geometric spreading loss is more problematic. The correct approach is to use a propagation model to compute the expected arrival pattern for the geometry and soundspeed field of interest. More often, simple rules of thumb are used. A conservative approach is to assume that each ray spreads spherically, as would be the case in a homogeneous, unbounded, lossless medium. The total power crossing any spherical surface surrounding the source must then be constant,

$$
P=4 \pi r_{0}^{2} I\left(r_{0}\right)=4 \pi r^{2} I(r)
$$


and the geometric spreading loss is

$$
T L_{g}=10 \log \left[I\left(r_{0}\right) / I(r)\right]=20 \log \left(r / r_{0}\right) \quad \mathrm{dB},
$$

where $r_{0}=1 \mathrm{~m}$ with the source level SL defined $1 \mathrm{~m}$ from the source. An alternate approach is to assume that (i) the total power summed over all ray paths spreads spherically out to a distance $r_{1}$ of the order of the water depth (10 km, say) and then spreads cylindrically (since the signal is confined between the top and bottom of the ocean), and (ii) the signal is apportioned among $n$ ray arrivals (reducing the intensity per ray arrival). For ranges in excess of several convergence zones, the result is

$$
T L_{g}=20 \log \left(r_{1} / r_{0}\right)+10 \log \left(r / r_{1}\right)+10 \log (n) \quad \mathrm{dB}
$$

It was shown that the number of ray arrivals increases linearly with range. The rate of increase is not necessarily sufficiently rapid to give spherical spreading. For the temperate sound-speed profile, $n=0.02 r(r$ in $\mathrm{km})$. At $1 \mathrm{Mm}$ range, spherical spreading gives $T L_{g}=$ $120 \mathrm{~dB}$, and with $n=0.02 r$ gives $T L_{g}=113 \mathrm{~dB}$.

An estimation of transmission loss in the LOAPEX experiment is implemented with consideration only the water column attenuation and spreading loss, which is shown in Figure 4-10.

\subsubsection{Estimation of Acoustic Wave Intensity Recorded from VLA}

The power spectra density of the sound pressure at different stations are estimated after the hydrophone's calibration, which are shown in Figure 4-11. The detail of the VLA calibration is in Appendix E. The Hanning window is applied here to compute the spectrum of six minute long time period signal. The power spectra density verify the previous rough estimation of the acoustic wave intensity for different distances. The sound pressure level at each station is roughly in the same order of level of estimations from Figure 4-10. 


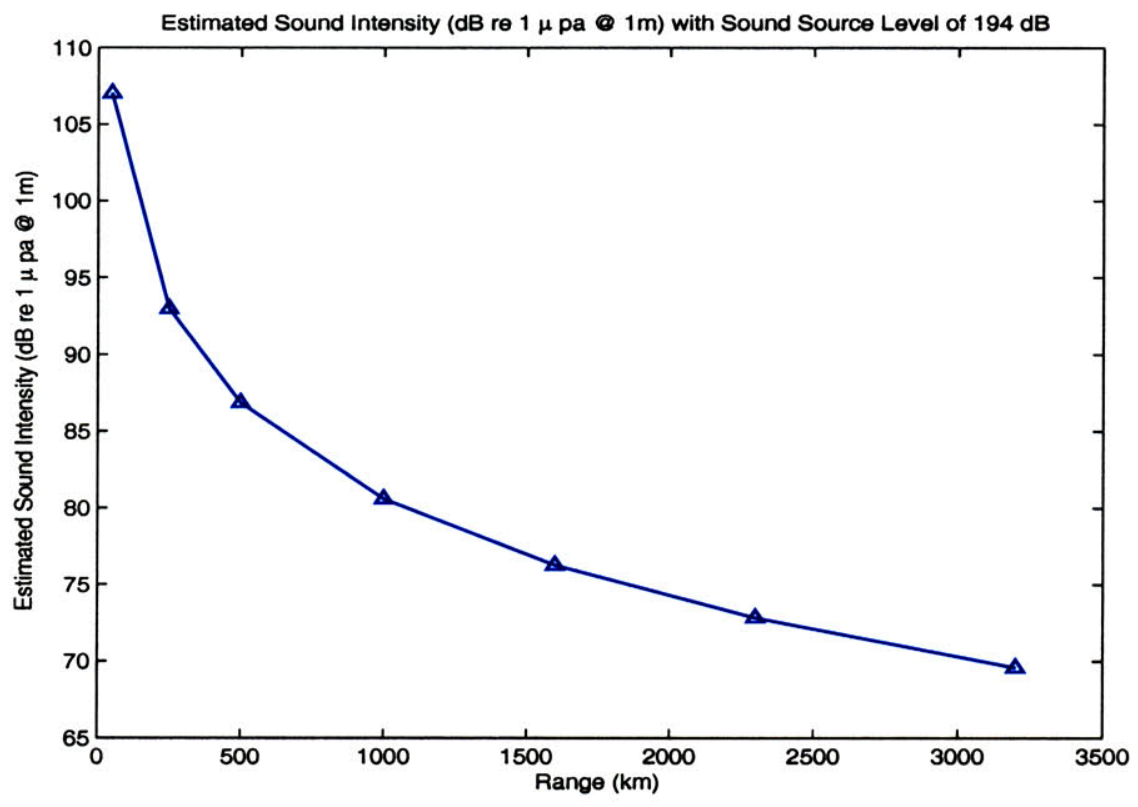

Figure 4-10: The estimated intensity recorded at VLA for LOAPEX transmissions. The spreading loss calculations for a single ray conservatively assume pure spherical spreading in first $10 \mathrm{~km}$ then cylindrical spreading afterward. Attenuation is calculated for the North Pacific Ocean using Lovett $(\mathrm{A}=0.055)$.

\subsubsection{Mean Wave Front Intensity}

The mean wave front intensity at each station is estimated with doppler correction. We first did coherent averaging of each group transmission which includes $10 \mathrm{M}$-sequence periods ${ }^{1}$. Then the incoherent average is implemented for all the groups. The mean wave front intensity with absolute acoustic wave intensity are shown in Figure 4-12 in unit of $\mathrm{dB}$ re $1 \mu \mathrm{Pa} @ 1 \mathrm{~m}$ with using the calibration data of both hydrophones and AVTOC instruments. All the estimations from previous three figures 4-10, 4-11, and 4-12 are in general well agreement, which verify our calibration results.

\subsection{Numerical Simulations}

To compare with observational results, there are three different kinds of simulations introduced and applied here, which are ray method, parabolic equation (PE) method, and (one-way) coupled normal mode.

\footnotetext{
${ }^{1}$ Because of the different sampling rates from the first stations (T50 - T1600) to the last two stations, the coherent averaging of last two stations are actually implemented for $36 \mathrm{M}$-sequence periods.
} 

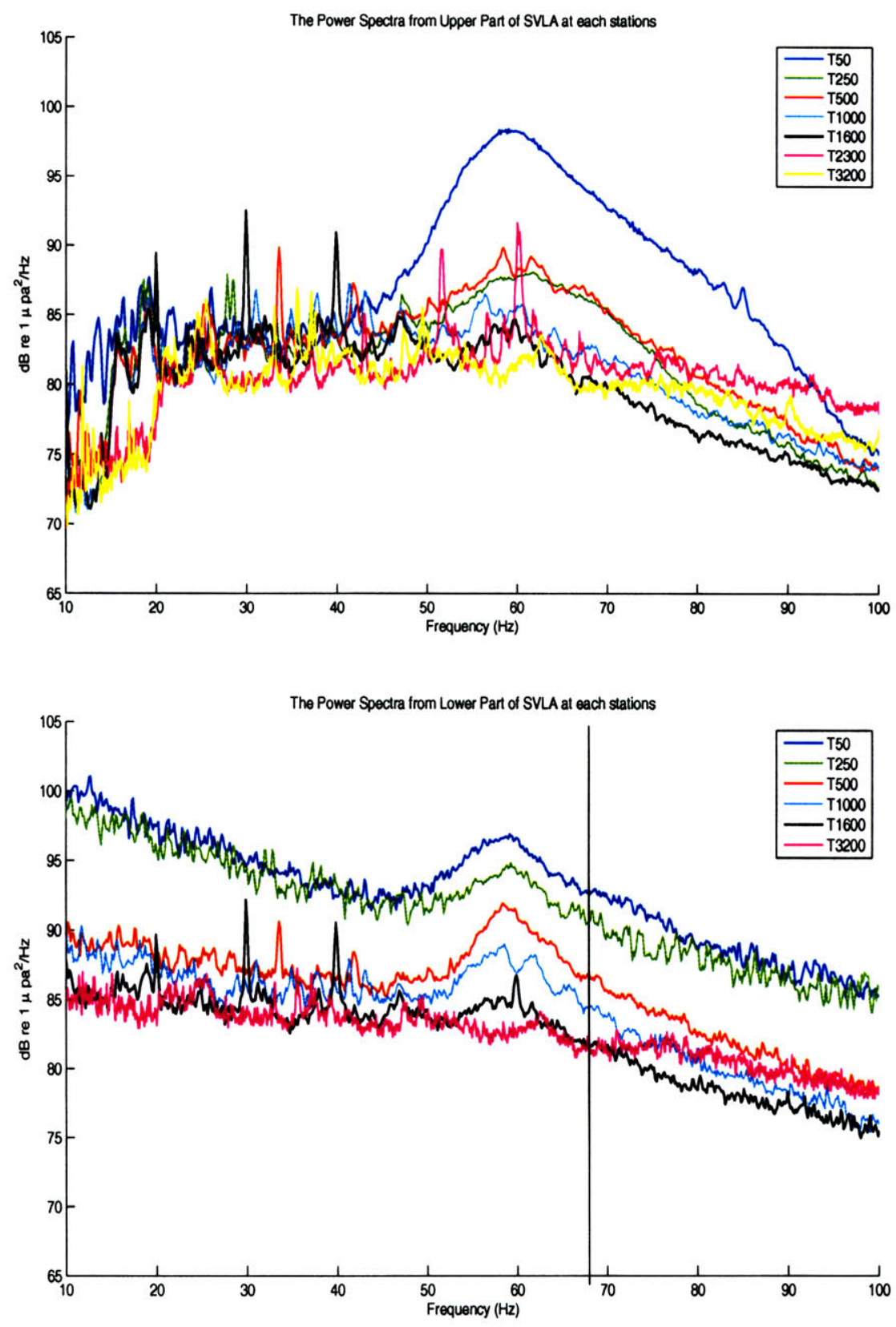

Figure 4-11: The power spectra density of the sound pressure at different stations. The upper panel indicate the data from upper SVLA, the lower panel indicates the data from lower SVLA. The vertical line in the lower panel indicates the carrier frequency of $68.2 \mathrm{~Hz}$. 

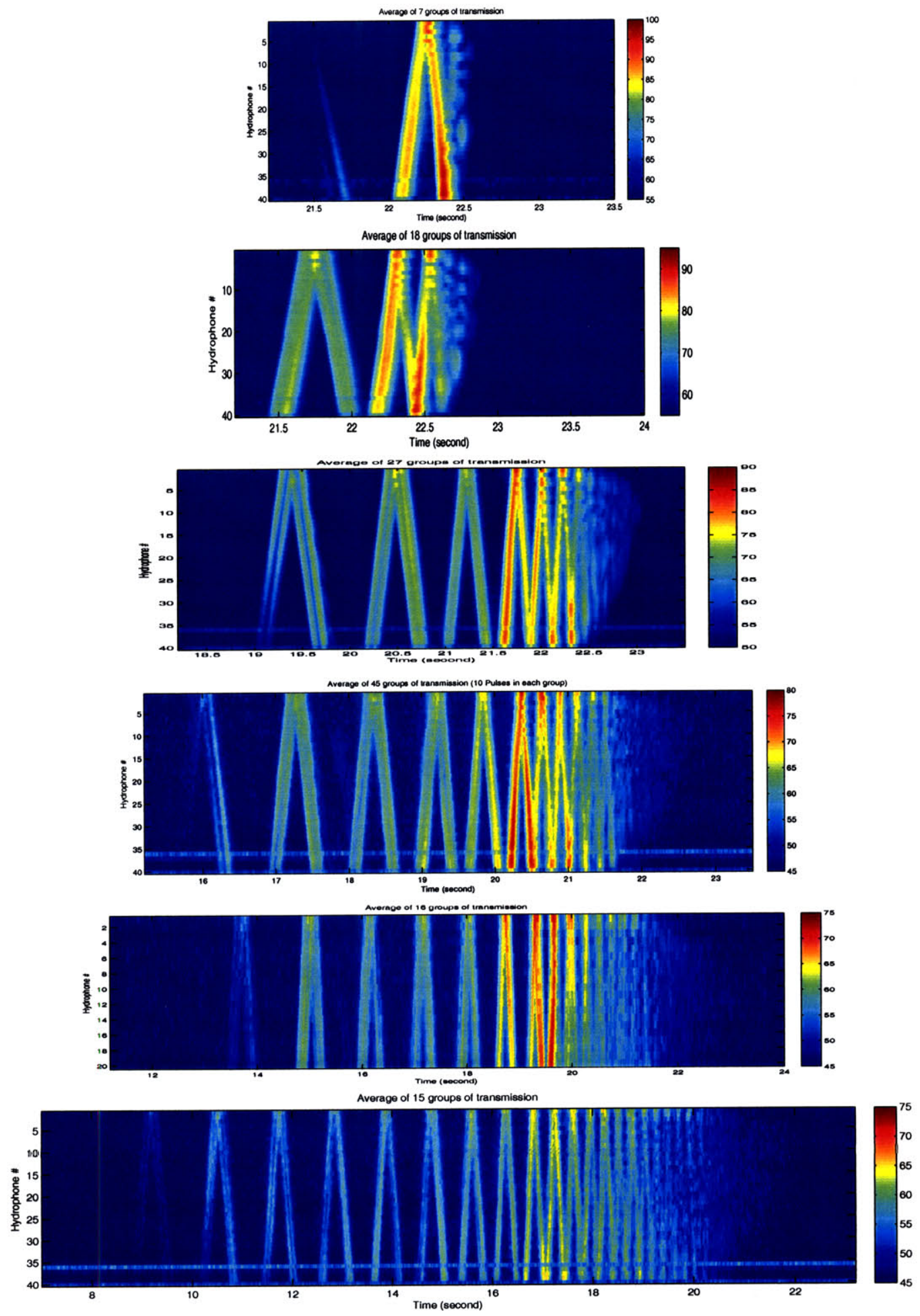

Figure 4-12: The mean wave front intensity at each station: T250 to T3200 from top to bottom respectively. The colorbars indicate the absolute sound intensity in unit of $\mathrm{dB}$ re $1 \mu \mathrm{Pa} @ 1 \mathrm{~m}$. 


\subsubsection{Ray method- Eigenray Code}

The ray code we implemented here is called eigenray code, which is a Fortran code originally developed by Bowlin et al. (1992)[5], and streamlined by Dushaw (1998)[27]. Although numerous ray tracing codes are available, this code has its advantage to achieve fast, accurate wavefront and eigenray travel time predictions at basin scale ranges (3 -5 M m). Two techniques are used to speed up the eigenray calculations: (i) To use an initial prediction with a small number of rays in order to define the range of ray angles that arrive near the depth of the receiver. (ii) To omit the calculation of travel time in the initial fan of rays. It is clear that a fast code will necessarily rely on look-up tables for sound speed, sound speed gradient, and other parameters. The methods using cubic spline interpolation and a lookup table allow sound speed and sound speed gradient to be calculated rapidly and accurately at arbitrary range and depth. The choice of the step size used in integrating the differential equations is critical, affecting the both the computation time and accuracy of the ray predications. For the range dependent case, the sound speed profile is interpolated linearly in the horizontal direction.

\subsubsection{Normal Modes Method - C-SNAP}

The normal modes code here we used is called the Coupled SACLANTCEN normal mode propagation loss model (C-SNAP)[38]. It is built as a range-dependent propagation loss model by Ferla, et al. on the base of a widely used and efficient range-independent normal mode code, SNAP, and a numerical solution technique for one-wave mode coupling obtained from KRAKEN. Despite the great achievements obtained with fast field and parabolic equation models, normal mode programs still remain a very efficient, simple, and practical tool for describing ocean acoustics in range-independent environments. C-SNAP generalize the range-independent problem to a range-dependent one by dividing the propagation path in a sequence of range-independent segments and using normal modes to represent the acoustic field in each segment. It takes advantage of a widely used finite-difference algorithm for solving the range-independent problem and make the assumption that the acoustic field is dominated by the outgoing component. The code incorporated a reliable algorithm for the automatic selection of the vertical grid spacing to be used for accurately marching the solution out in range. It also bypassed the calculation of mode coupling matrices 
and computed the mode coefficients in a new segment by projecting the pressure field onto the new mode set. To preserve accuracy, an energy-conserving matching condition is implemented at the coupling interfaces. One of its several prominent features which is worthy to emphasis here is that execution speed is relatively fast, and the overall time required to get a stable solution is mainly dependent on the choice of a single parameter: the number of range subdivisions.

\subsubsection{Parabolic Equation method - RAM}

The parabolic equation (PE) method is very effective for solving range-dependent ocean acoustics problems. The Range-dependent Acoustic Model (RAM) is based on the splitstep Padé solution[12, 13], which allows large range steps and is the most efficient PE algorithm that has been developed. Range dependence is handled accurately by applying an energy-conservation correction[14] as the acoustic parameters vary with range. An initial condition (or starting field) is constructed using the self-starter[11], which is an accurate and efficient approach based on the PE method.

The nurnerical solution of the parabolic wave equation involves repeatedly solving tridiagonal systems of equations. This key component of RAM has been optimized by minimizing the number of operations and by using a special elimination scheme that is efficient for problems involving variable ocean depth. The split-step Padé algorithm is based on rational function approximations. This code is originally written by Michael Collins. The specific package we used here is implemented in matlab as developed by Matt Dzieciuch.

Before running the simulations, the proper parameters for PE replica calculations were established. Of great importance were the $\Delta r$, or marching step interval, and $\Delta z$, the depth interval. These two parameters determined the granularity of the acoustic field calculation using PE. If these parameters were too large, the simulated pressure field would not be accurate. Too small, and too much time would be spent computing the field for the desired accuracy.

\subsubsection{Comparison between the different numerical simulations}

To investigate how the sound wave is scattered in the random medium, i.e. internal wave field, we used a stochastic internal wave model as described in Chapter 3 and propagated sound wave through it. In this thesis, all simulations with internal waves are set up with 
half GM energy, unless indicated otherwise.

Here we used the ray code to get accurate travel time estimation. Furthermore, as the ray code does not account for diffraction effects, comparison between the ray and other numerical simulations (such as PE and Normal Modes codes) indicates the diffraction effects is involved. PE simulations (range independent/ dependent) are very efficient and the only available method to study the sound wave propagation through internal wave fields. The normal modes method is the most accurate full wave equation method, used here to verify the PE simulation result. The simulations with these methods are displayed at each station in Figure from 4-19 to 4-24.

In each figure, we show three panels with $\mathrm{PE}$ (RAM) simulation without internal waves (upper), CSNAP simulation without internal waves (center), and PE (RAM) simulations with internal waves (bottom). For the simulations without internal waves field, the PE (RAM) and CSNAP show very similar results in both early arrival wave fronts and finale region for the first few stations. But for the longer range stations, such as T1600, T2300, and T3200, there are some discrepancies in the post finale region. The reason for this discrepancy might be due to the accuracy of the PE (RAM) simulation. It is expected that reducing step size in both horizontal and vertical direction will have better agreement with CSNAP's result. For the simulation including internal wave scattering, as we expect, there is energy extending in both horizontal and vertical direction. But in the post-finale region, the internal wave scattering seems to smear the energy in both vertical and horizontal direction instead of pushing energy extending further in the time axis (such as station T1600 and T2300), which is an interesting finding for this off-axis sound source transmission.

\subsection{Comparison between Observations and Numerical Sim- ulations}

\subsubsection{Mean Wave Front Intensity in the Finale Region}

The acoustic data at different ranges are processed with procedures of demodulation and pulse compressing for off-axis transmissions. Doppler shift finding and correction are applied to remove the relative motion effect of the sound source and receiver moorings. The mean intensity of the wave fronts were estimated first by coherently averaging for continuous pulses with doppler correction, and then incoherently averaging among different transmis- 

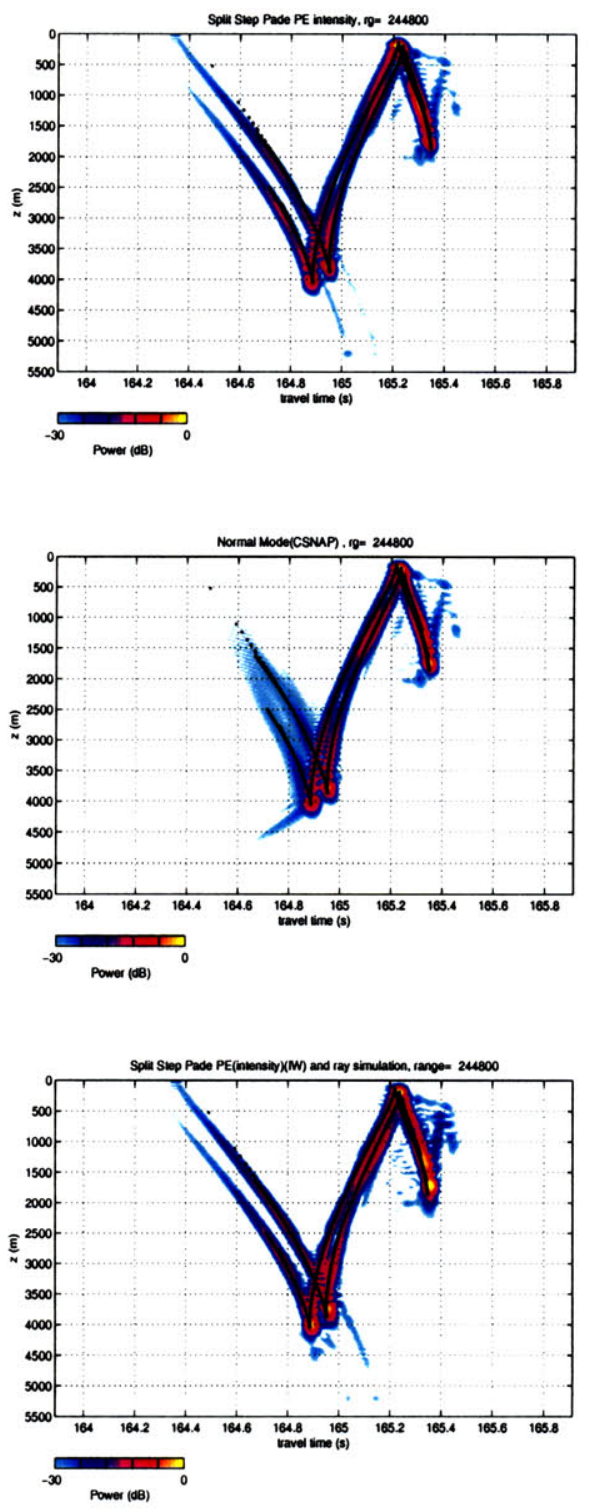

Figure 4-13: The simulations of LOAPEX station T250 transmission. From top to bottom, the three panels are PE (RAM) simulations without internal wave, CSNAP simulations without internal wave, and the PE (RAM) simulations with internal wave, respectively. In each simulation, the eigenray simulations are overlapped on with black dot marks. 

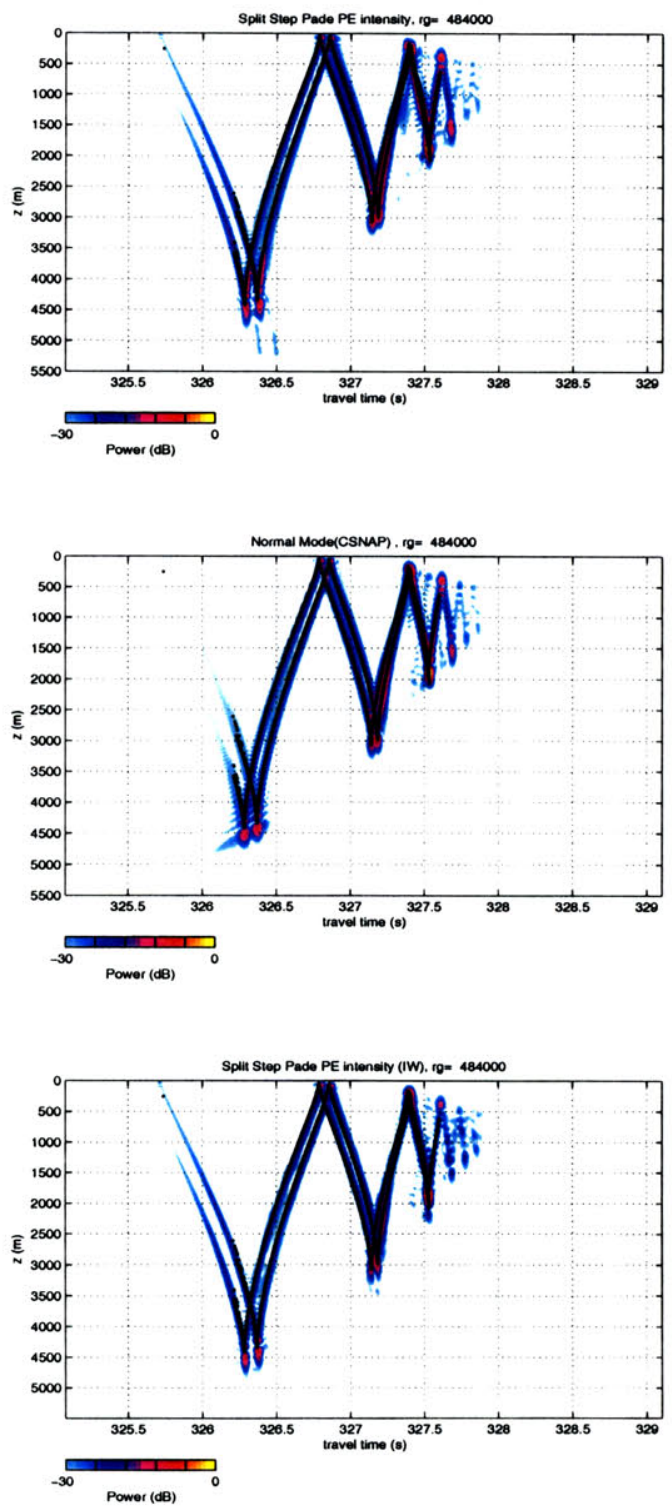

Figure 4-14: The simulations of LOAPEX station T500 transmission. From top to bottom, the three panels are PE (RAM) simulations without internal wave, CSNAP simulations without internal wave, and the PE (RAM) simulations with internal wave, respectively. In each simulation, the eigenray simulations are overlapped on with black dot marks. 

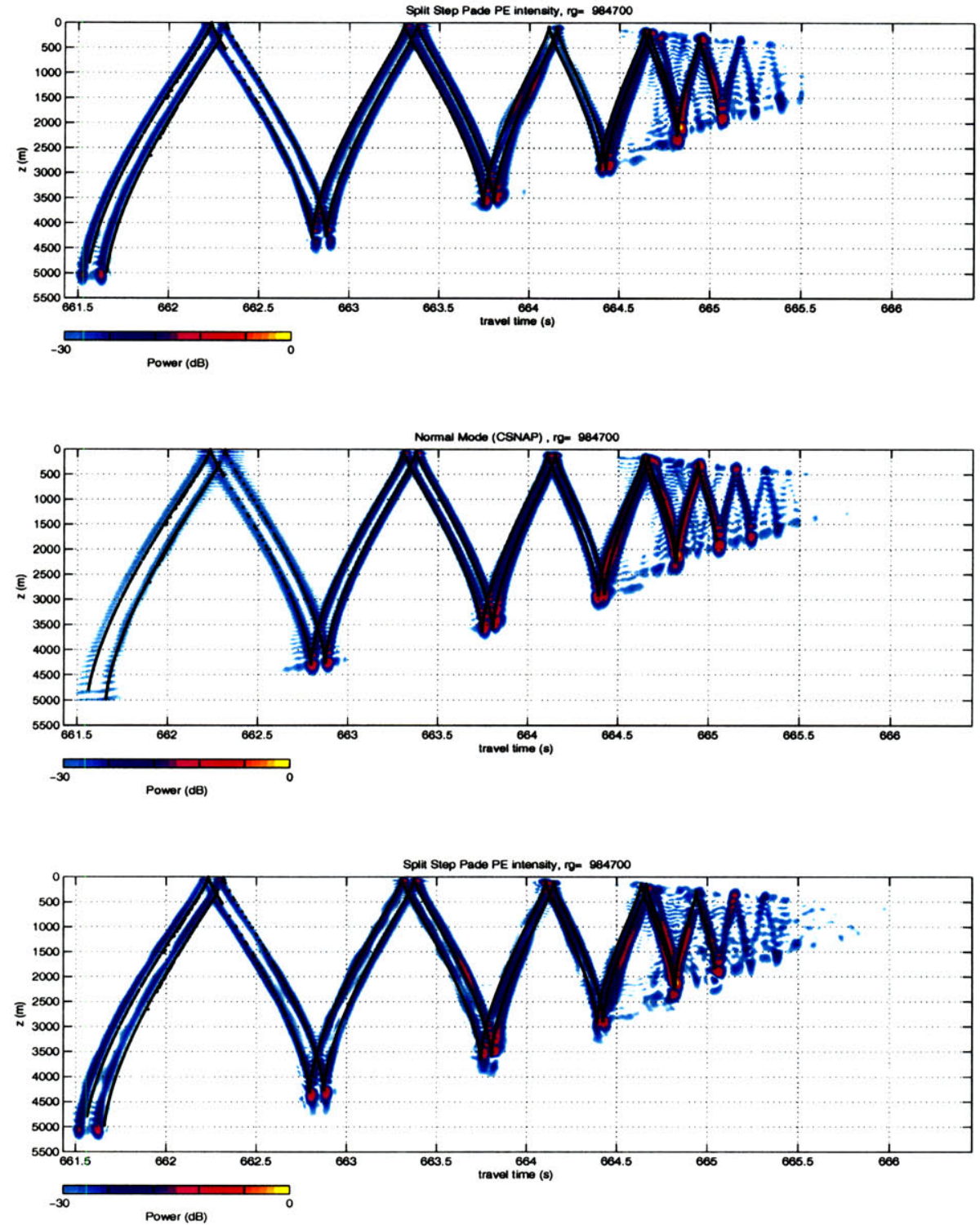

Figure 4-15: The simulations of LOAPEX station T1000 transmission. From top to bottom, the three panels are PE (RAM) simulations without internal wave, CSNAP simulations without internal wave, and the PE (RAM) simulations with internal wave, respectively. In each simulation, the eigenray simulations are overlapped on with black dot marks. 

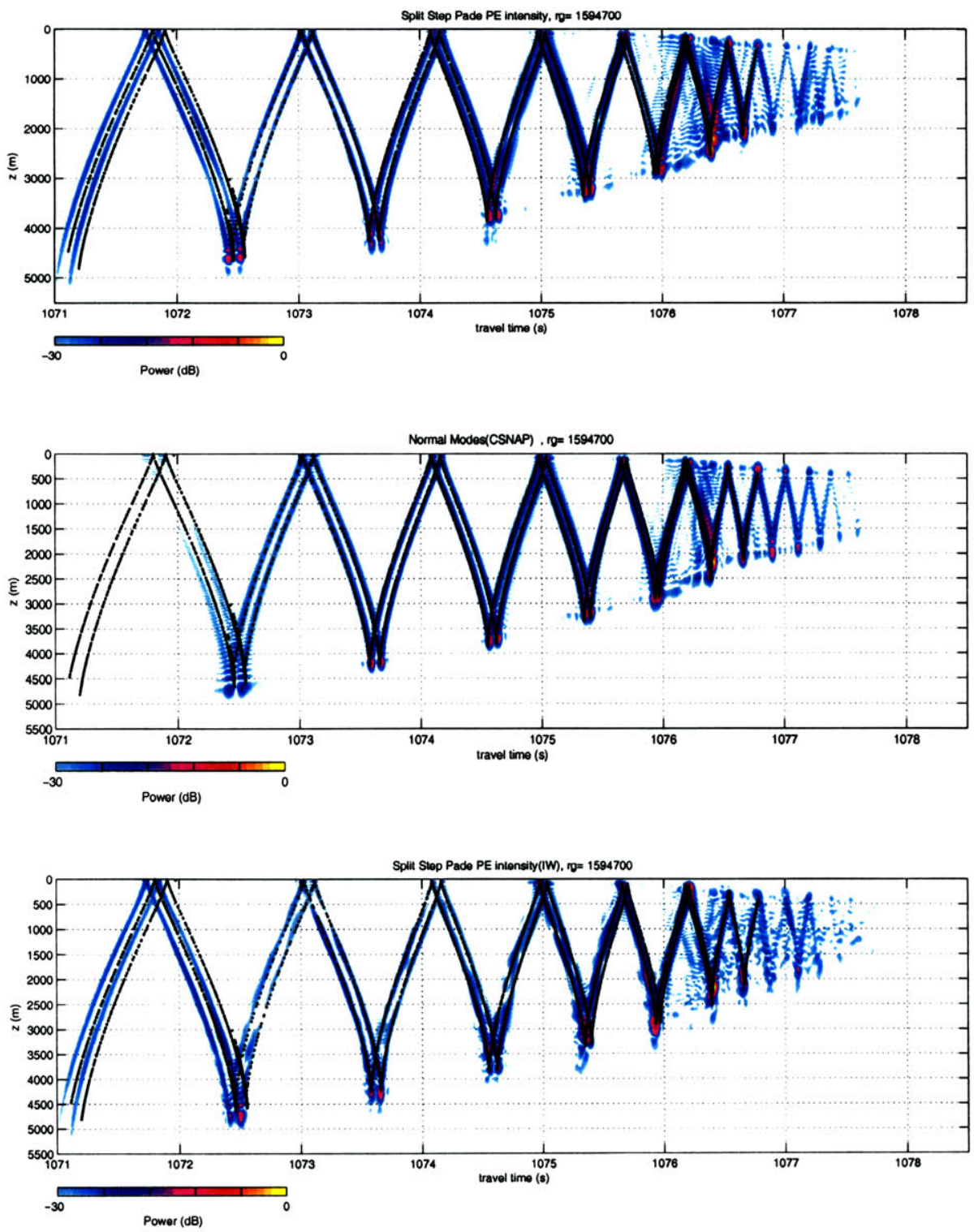

Figure 4-16: The simulations of LOAPEX station T1600 transmission. From top to bottom, the three panels are PE (RAM) simulations without internal wave, CSNAP simulations without internal wave, and the PE (RAM) simulations with internal wave, respectively. In each simulation, the eigenray simulations are overlapped on with black dot marks. 

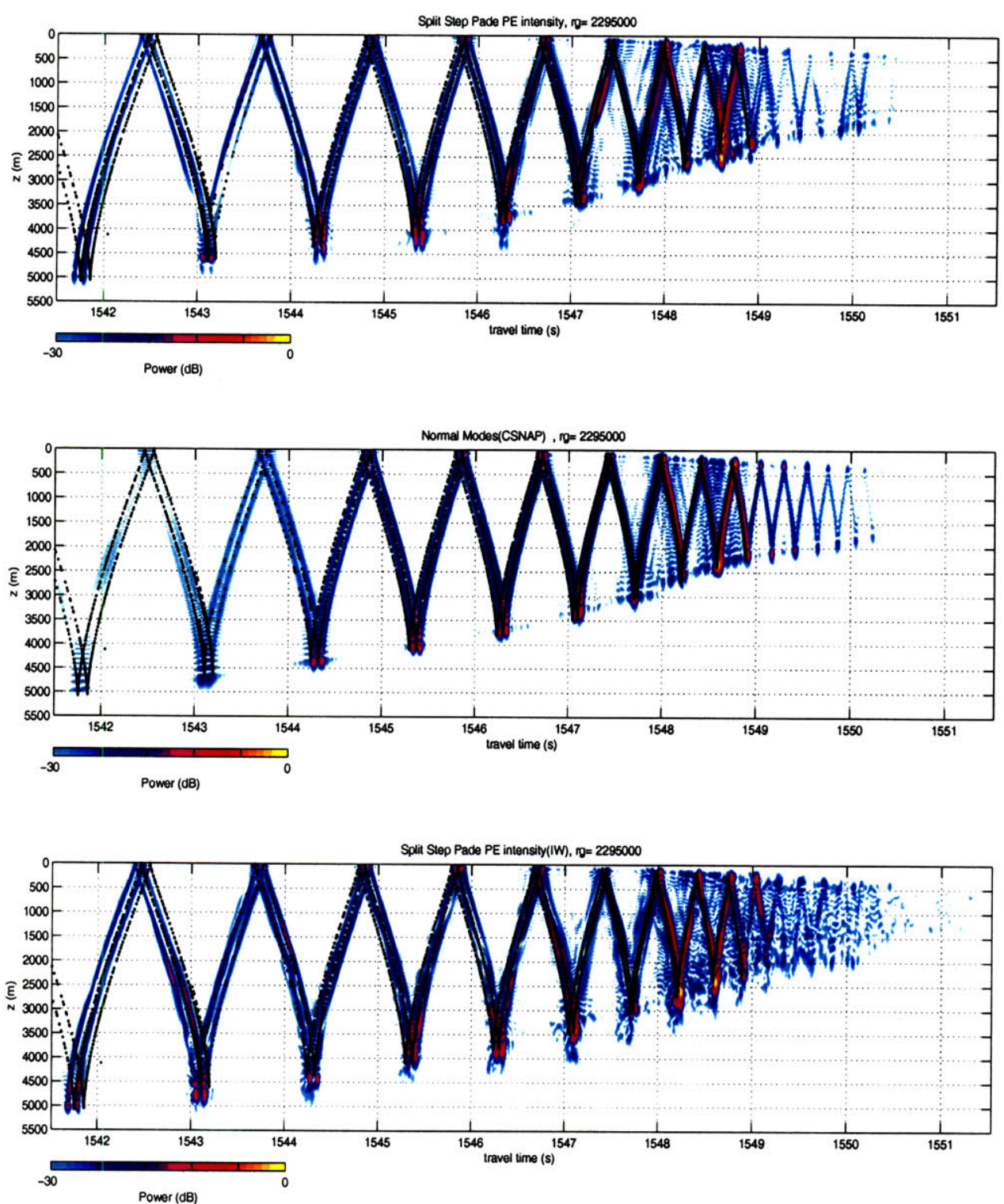

Figure 4-17: The simulations of LOAPEX station T2300 transmission. From top to bottom, the three panels are PE (RAM) simulations without internal wave, CSNAP simulations without internal wave, and the PE (RAM) simulations with internal wave, respectively. In each simulation, the eigenray simulations are overlapped on with black dot marks. 

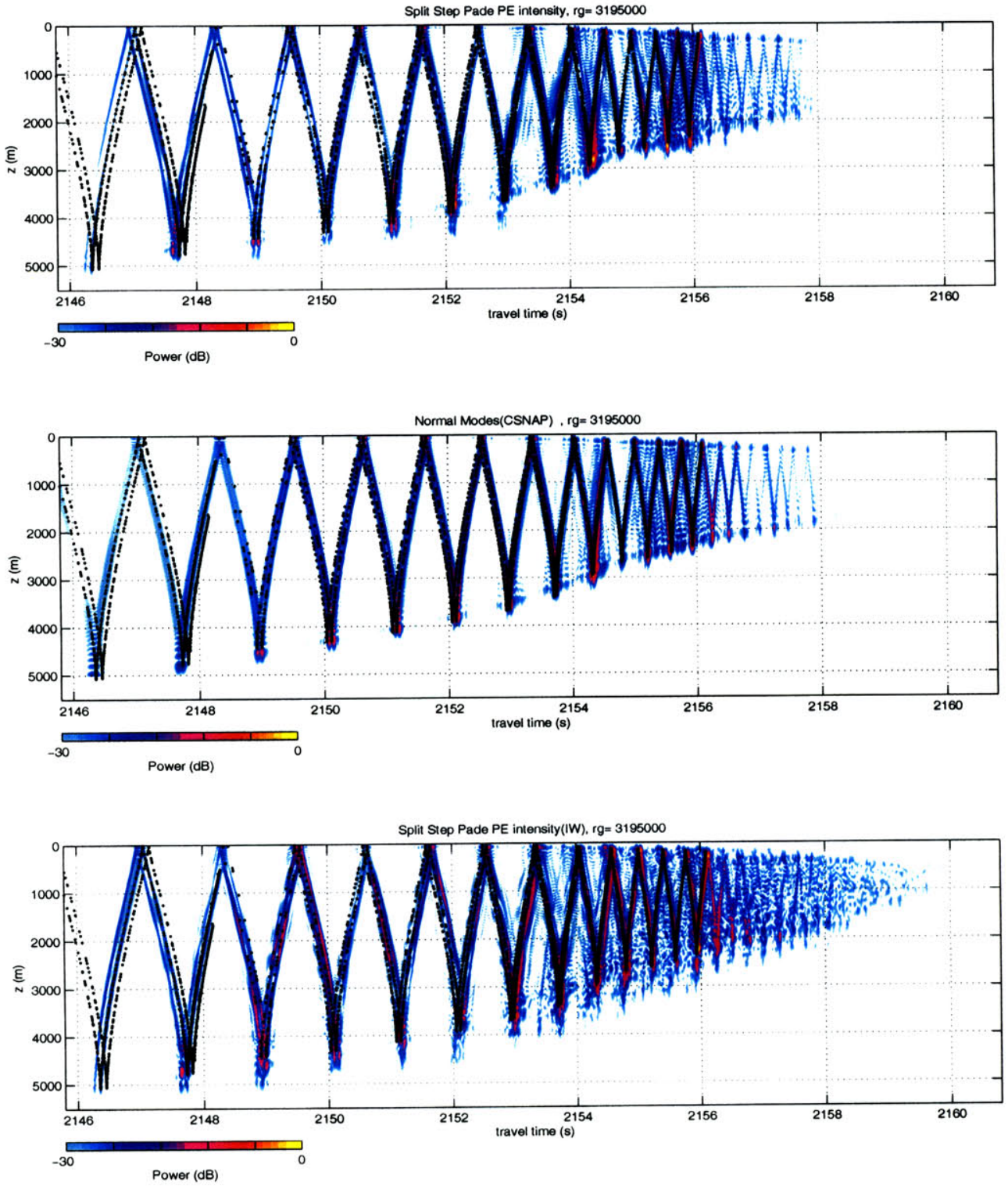

Figure 4-18: The simulations of LOAPEX station T3200 transmission. From top to bottom, the three panels are PE (RAM) simulations without internal wave, CSNAP simulations without internal wave, and the PE (RAM) simulations with internal wave, respectively. In each simulation, the eigenray simulations are overlapped on with black dot marks. 
sions. For the off-axis transmissions, signal to noise ratio (SNR) of the wavefronts range between $40 \mathrm{~dB}$ (for less than $2 \mathrm{M}$ m stations) to $25 \mathrm{~dB}$ (for further stations:T2300 and T3200.) The observations of mean intensity time-fronts are compared to the deterministic ray, Parabolic Equation (PE) (with/without internal waves) and (one-way coupled)normal mode calculations. From Fig. 4-19 to Fig. 4-24, we show the comparison between the observations and numerical simulations with/without the internal wave field.

The upper panel of each figure, shows the comparison between the ray prediction and observation result. The wave fronts seen sweeping across the array in the early part of the reception have one-to-one correspondence with the predicted wave fronts. However, the measured and predicted arrival patterns are not identical. The most striking discrepancy is in the times at which the acoustic receptions end, i.e., the final cutoffs, which have been already described in terms of the diffraction effect.

Comparison of the observations (upper panel) with the center and lower panel of each figure, shows that simulations with internal wave are in better agreement with observations in the post-finale region. However, there are some still discrepancies which need to be further examined for both simulations and data processing.

\subsubsection{LOAPEX Time Extension into the Finale Region}

In general, the LOAPEX observations show that there is significant amount of energy ensonified in the shadow zone region at each station. These shadow zone arrivals are beyond the geometry of acoustic prediction, and we know it is due to the sound wave diffraction and scattering. To investigate the roles of these two effects, let us first examine the mean intensity fluctuation averaged along the hydrophone receiver depths (from $350 \mathrm{~m}$ to 1715 $\mathrm{m})$,

$$
\bar{I}(t, z)=\frac{1}{N_{z}} \sum_{j=1}^{N_{z}} I(t, z, R)
$$

This average intensity at each station (range) is shown in Figure 4-25:

Each panel in Figure 4-25 shows the comparison of observation data and simulations . From top to bottom, the five panels are station T250, T500, T1000, T1600, T2300, and T3200 respectively. In each panel, there are four line indicating the LOAPEX observation, CSNAP simulation, PE RAM simulation with internal wave, and last ray arrival. In general, 

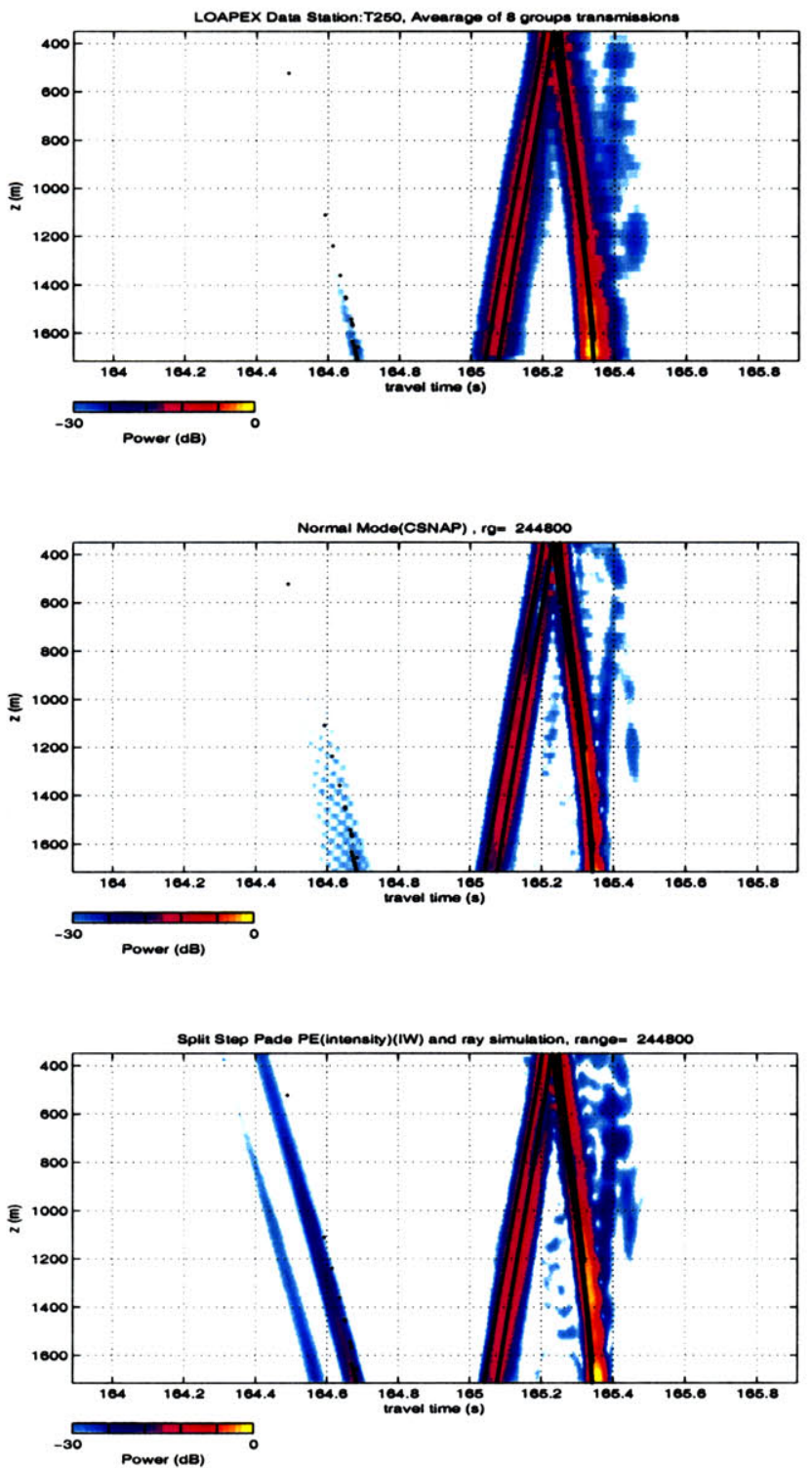

Figure 4-19: The comparison of observation data and simulations of LOAPEX station T250. From top to bottom, the three panels are LOAPEX transmission data, CSNAP simulations without internal wave, and the PE (RAM) simulations with internal wave, respectively. In each panel, the eigenray simulations are overlapped on with black dot marks. 

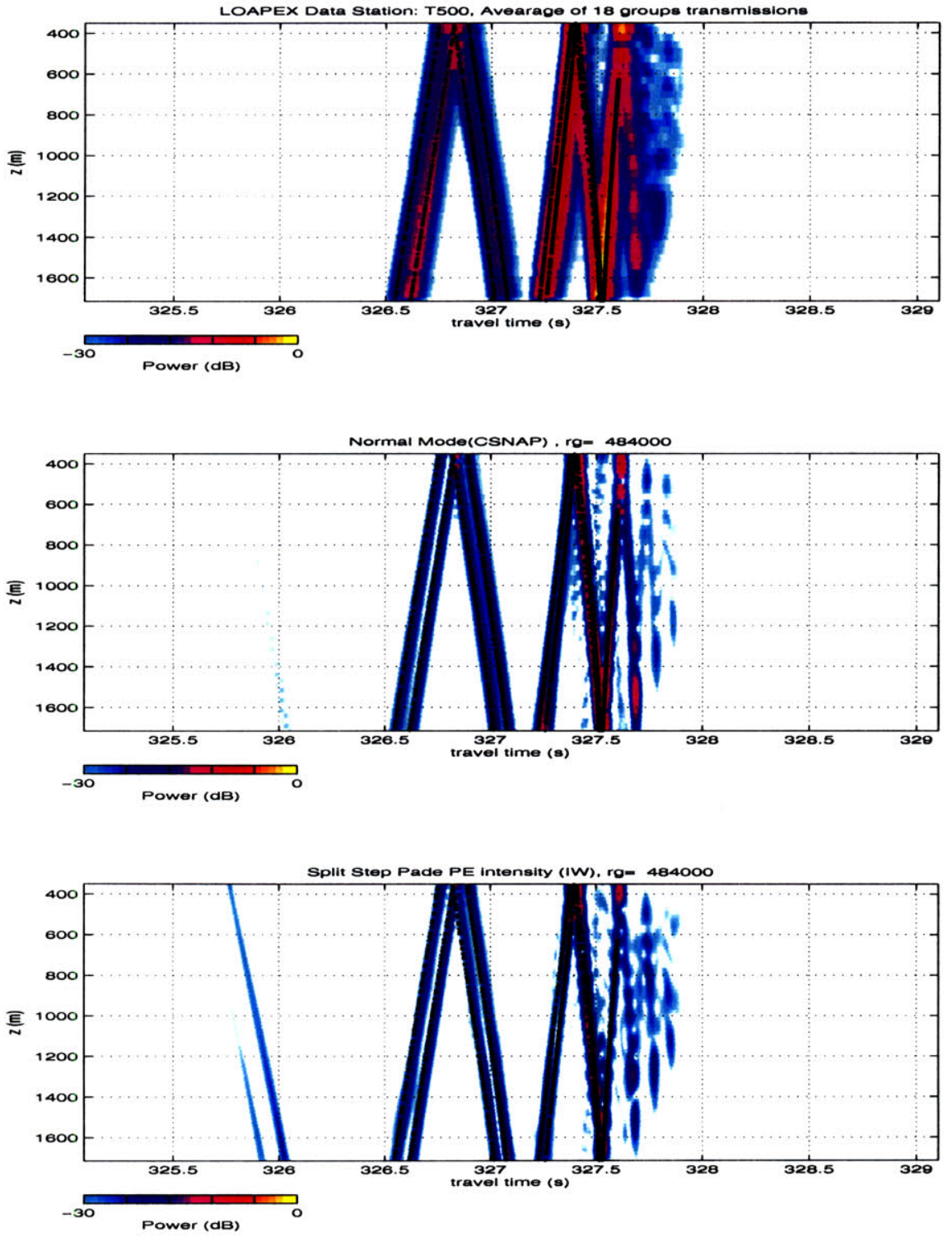

Figure 4-20: The comparison of observation data and simulations of LOAPEX station T500. From top to bottom, the three panels are LOAPEX transmission data, CSNAP simulations without internal wave, and the PE (RAM) simulations with internal wave, respectively. In each panel, the eigenray simulations are overlapped on with black dot marks. 

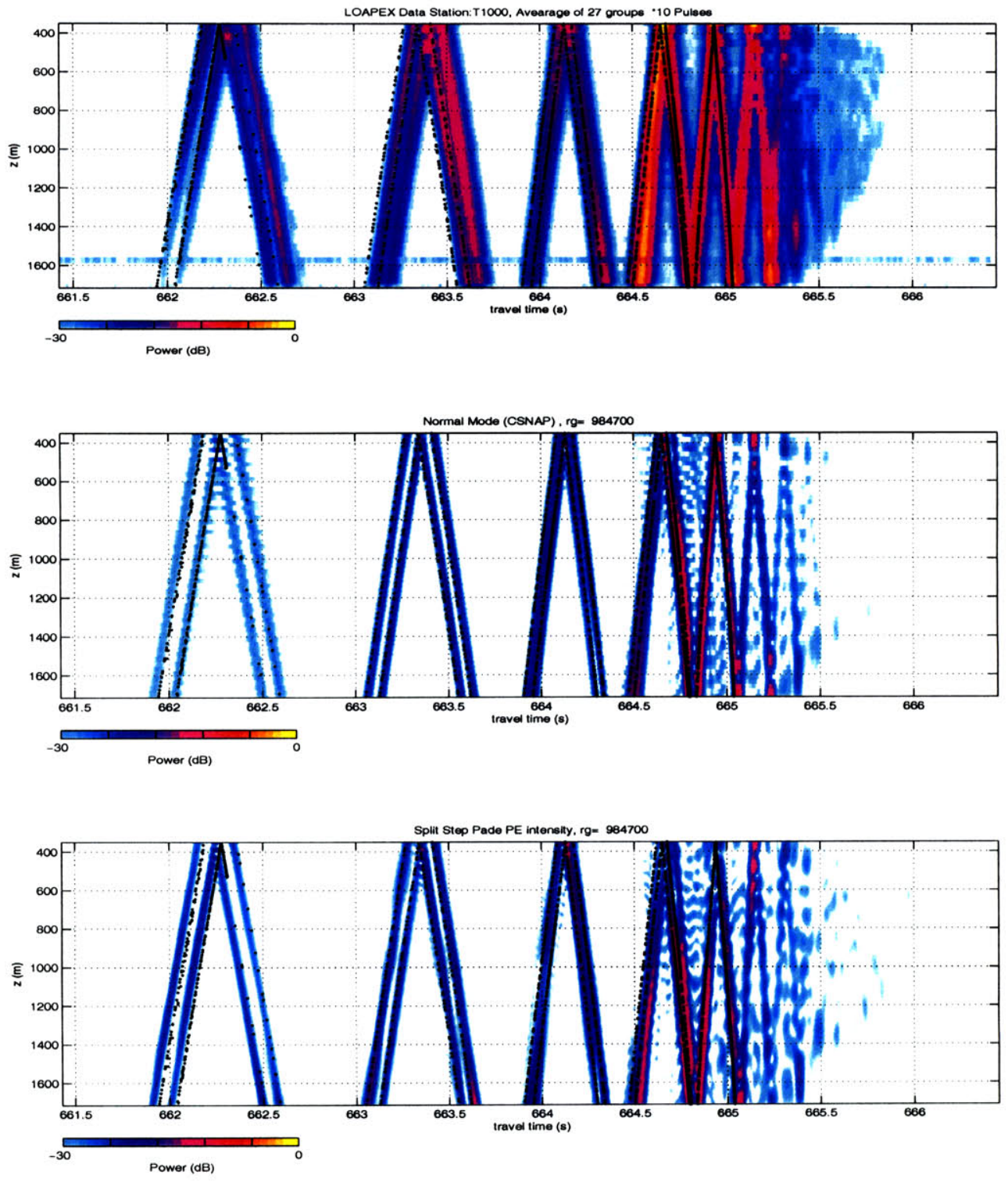

Figure 4-21: The comparison of observation data and simulations of LOAPEX station T1000. From top to bottom, the three panels are LOAPEX transmission data, CSNAP simulations without internal wave, and the PE (RAM) simulations with internal wave, respectively. In each panel, the eigenray simulations are overlapped on with black dot marks. 

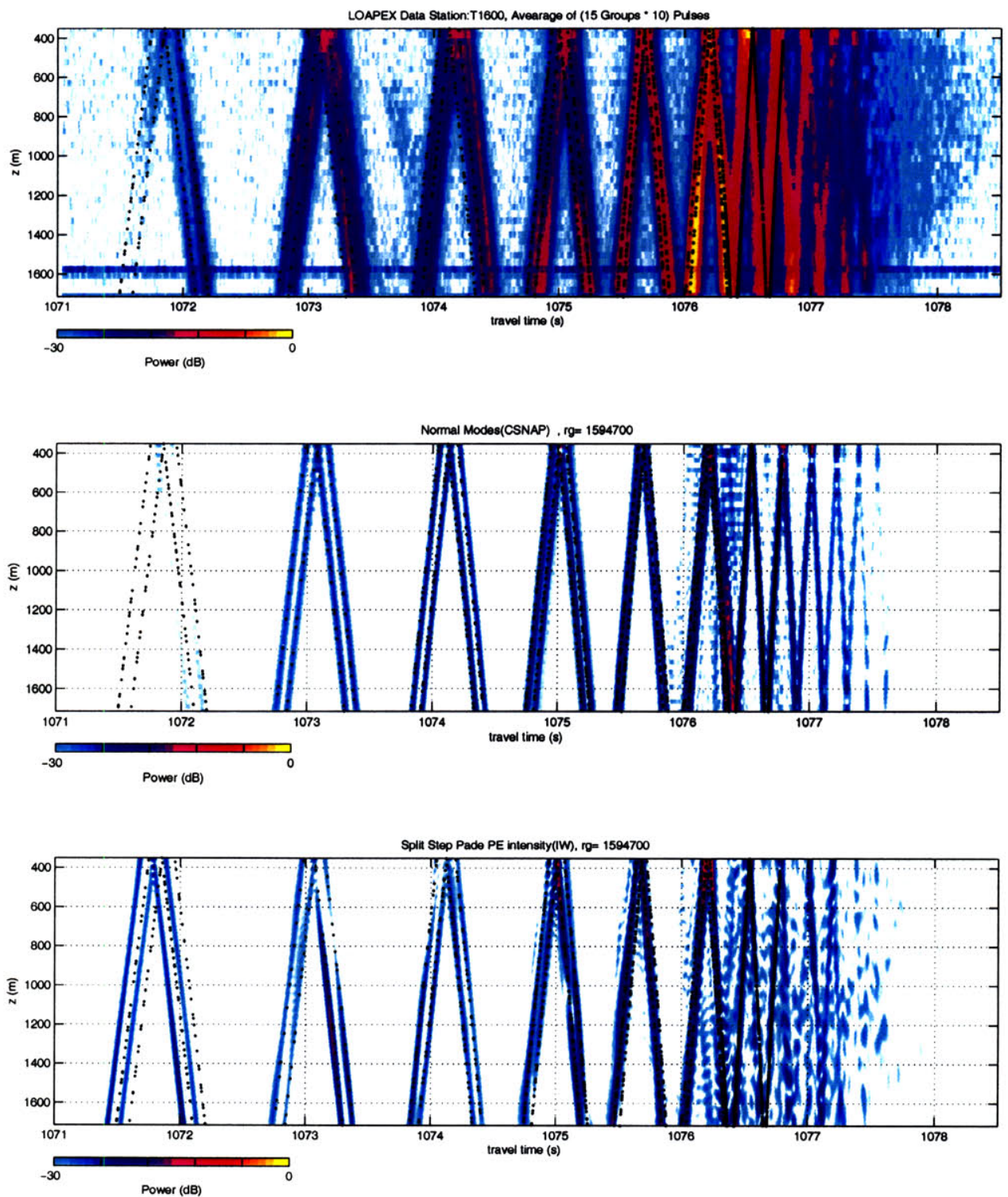

Figure 4-22: The comparison of observation data and simulations of LOAPEX station T1600. From top to bottom, the three panels are LOAPEX transmission data, CSNAP simulations without internal wave, and the PE (RAM) simulations with internal wave, respectively. In each panel, the eigenray simulations are overlapped on with black dot marks. 

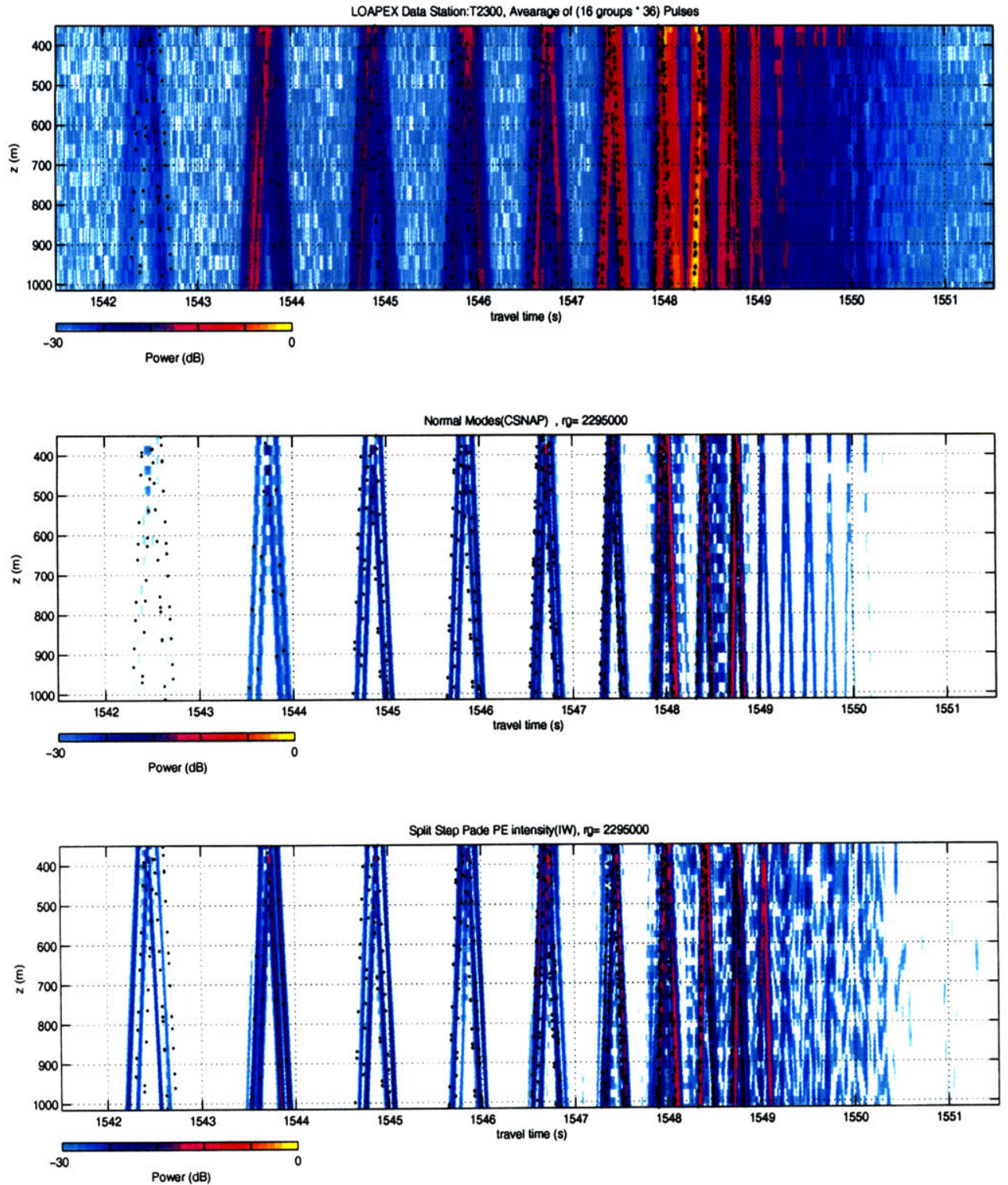

Figure 4-23: The comparison of observation data and simulations of LOAPEX station T2300. From top to bottom, the three panels are LOAPEX transmission data, CSNAP simulations without internal wave, and the PE (RAM) simulations with internal wave, respectively. In each panel, the eigenray simulations are overlapped on with black dot marks. 

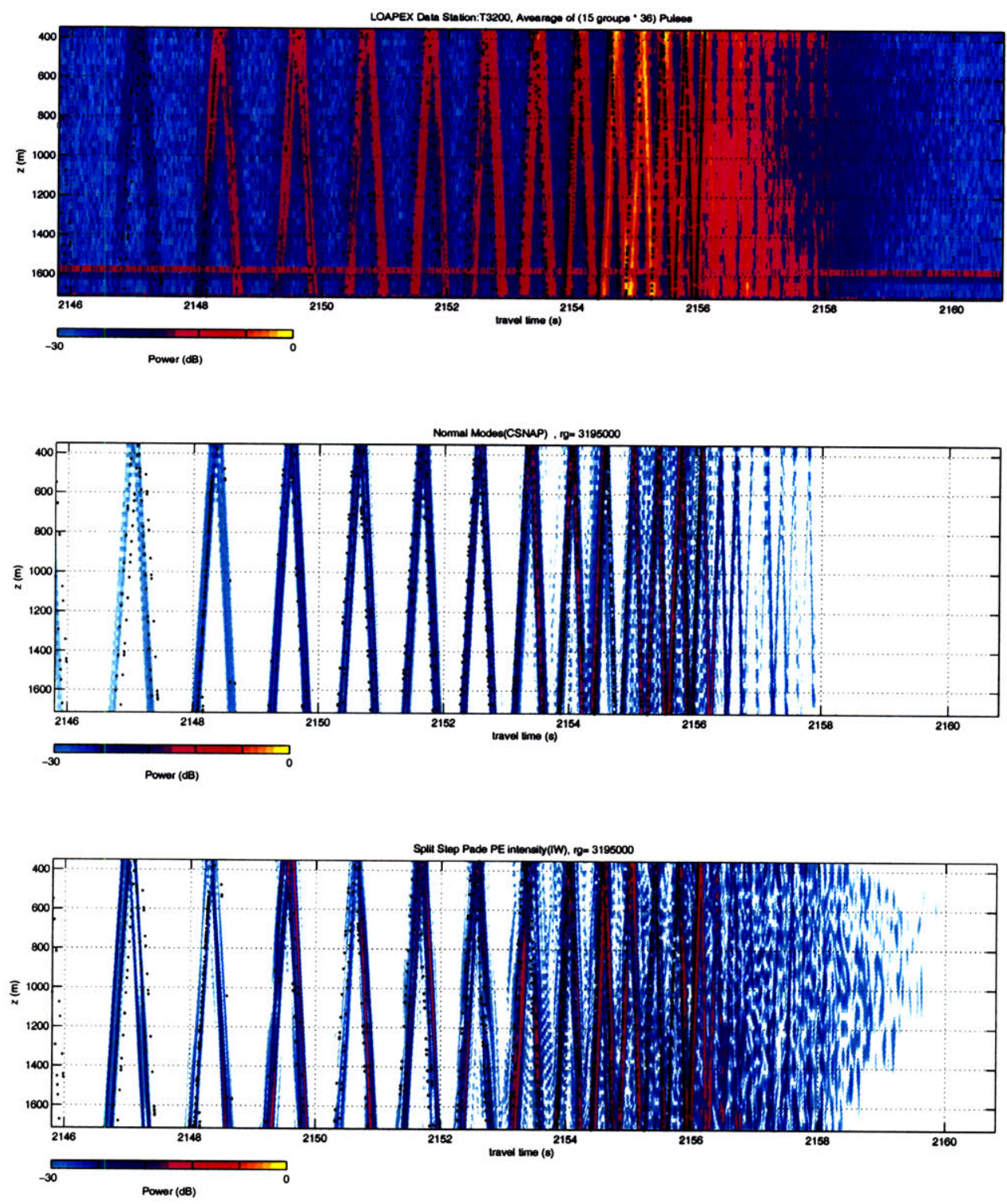

Figure 4-24: The comparison of observation data and simulations of LOAPEX station T3200. From top to bottom, the three panels are LOAPEX transmission data, CSNAP simulations without internal wave, and the PE (RAM) simulations with internal wave, respectively. In each panel, the eigenray simulations are overlapped on with black dot marks. 

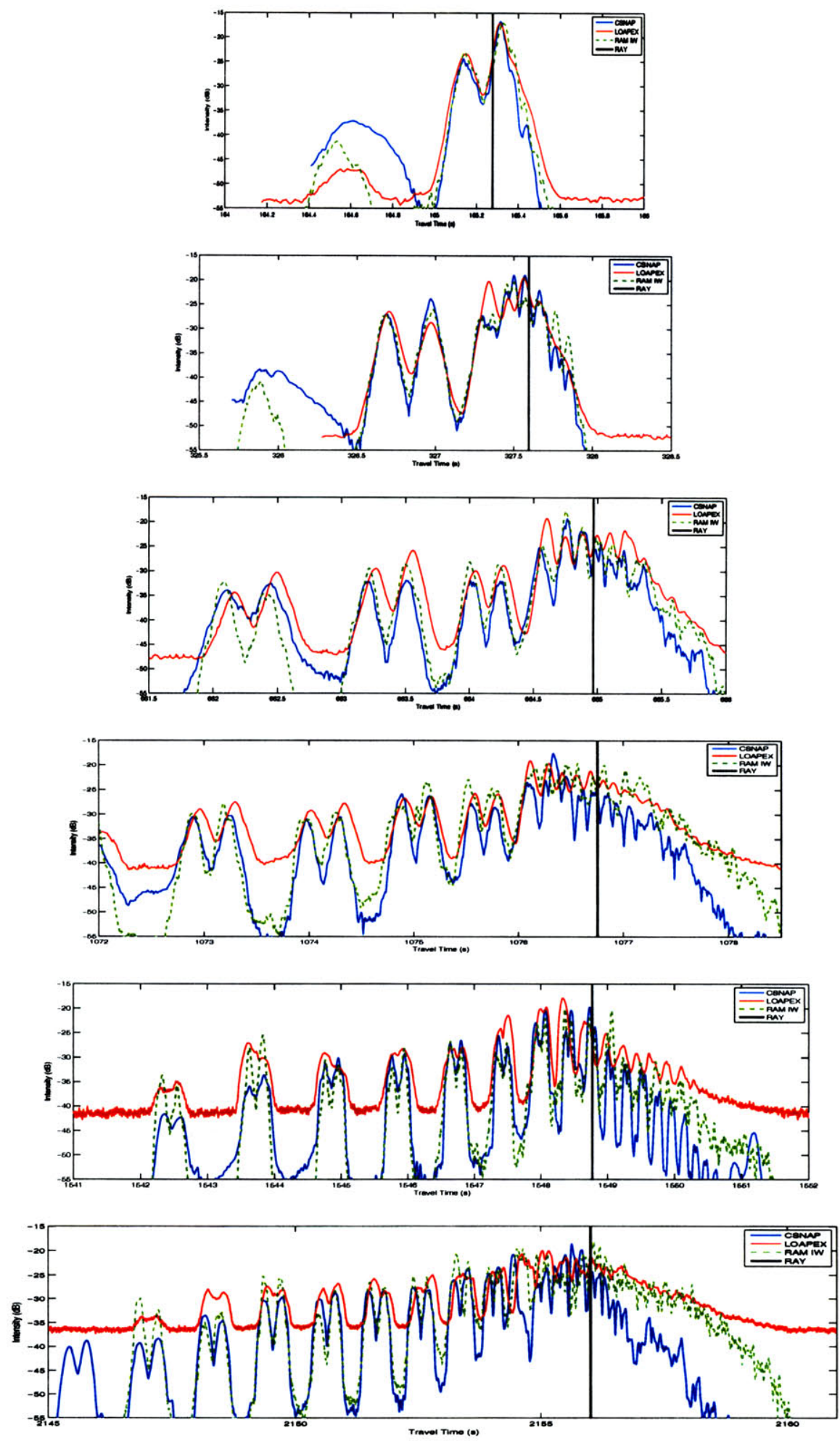

Figure 4-25: The comparison of observation data and simulations . From top to bottom, the five panels are station T250, T500, T1000, T1600, T2300, and T3200 respectively. In each panel, there are four lines indicating the LOAPEX observation, CSNAP simulation, PE RAM simulation with internal wave, and last ray arrival. 
the comparisons show very good agreement in the early arrival region for all the stations (up to $3200 \mathrm{~km}$ ). In the finale and even post-finale region (after last ray arrival), the agreement are still satisfactory in general for the first four stations (almost up to $1600 \mathrm{~km}$ ). As being expected, in general, diffraction is dominant effect at short ranges. Even the simulations with internal waves scattering do not differ that much from the observations and simulations without internal wave effects. However, for the longer ranges, such as stations T2300 and $\mathrm{T} 3200$, in the finale and post-finale region, there are apparent discrepancy between the observations and simulations, which indicate that the diffraction effect is no longer dominant and that the scattering effects are starting to contribute more. However, it is interesting to observe that the internal wave scattering effect can not fully explain this discrepancy. The alternative explanations include:

1. The average along the vertical direction might smooth out the internal wave scattering effect, since we know the scattering usually cause more energy extended in the vertical direction than in horizontal direction.

2. Internal wave scattering effect should be simulated with more realizations (Monte Carlo) to get more accurate statistical result, here we only have one realization.

3. Internal wave energy level might be another issue here. All the simulations with internal wave scattering are implemented with half GM energy. The preliminary result of LOAPEX deep arrivals (Wolfson 2007)[80] indicates 1.3 GM energy is closer to the observations.

4. The lower angle arrivals (lower acoustic modes) at sound channel depth might be greatly impacted by the large or mesoscale random medium effect other internal waves in these basin scale range transmissions.

Another measure of the scattering effect is time spreading after last ray arrival. Time spreading is defined as the following and its result is shown in Figure 4-26:

$$
\Delta t(z, R)=\left(\frac{\int_{t_{0}}^{\infty}\left(t-t_{0}\right)^{2}[I(t, z, R)-N(z, R)] d t}{\int_{t_{0}}^{\infty}[I(t, z, R)-N(z, R)] d t}\right)^{1 / 2}
$$

where $t_{0}$ is the time of the last ray arrival in the finale region. $t_{0}$ is indicated with vertical straight line in Figure 4-25. $N(z, R)$ is the noise level at different depth $(z)$ for each station $(R)$. The time spreading represents the spreading of the energy after the last ray 
arrival in the finale region. Figure 4-26 shows the time spreading of LOAPEX observations and simulations with/without internal waves, which is calculated based on this formula. As shown in the figure, time spreading is a function of depth and range, whichincreases as the transmission distance gets further and depth gets shallower. It is closely related to scattering effect induced by both the internal wave and other random medium fluctuations in the ocean.

The scale to the range of the time-spreading can actually quantify the relative contributions of diffraction and scattering to the time extension of the finale. In figure 4-27, the average of time spreading function along the depth (defined by Eq. 4.8 ) is computed for the LOAPEX observation, simulation with/without internal waves. At short ranges, the time spreading function scales linearly with range and at longer ranges it should scale like $3 / 2$ to the range with the scattering effect playing a dominant role.

In order to compare to the $3 / 2$ and linear scale, two reference scale curves are shown as dash lines in Figure 4-27. For the loapex data (the red line), it seems much closer to $3 / 2$ scale only if there were more spreading in station T3200. But as we know, for the case of station T3200, SNR is relatively low, which could be the reason not to get accurate estimation.

For the simulations with (cyan)/without (black) internal waves, they are apparently off from both scales at stations T1000, T1600, and T2300. If both stations T1000 and T1600 had less time spreading, and T2300 had more time spreading, then the simulation with IW would be $3 / 2$ scale, and simulation without IW would be much closer to linear scale. Actually the sound channel depths are actually changing along the transmission path. In Figure 4-2, it shows the shallowest sound channel depth at station T1000 and station T1600, which means the sound source were closer to the sound channel depth than other stations. However, for station T2300, the sound channel depth is the deepest one. For the case of off-axis sound source, the closer the sound source is to the sound channel, the more lower-angle energy will be excited, which accounts for the more time-spreading for station T1000 and T1600 of the simulation results here. The time-spreading scale of the simulation without internal wave is actually linear, taking account of the changing sound channel depth, which is the case with diffraction effect only. Because the sound channel depth was varied along the transmission path during the LOAPEX experiment, it makes this problem more complicated than the constant sound channel depth. Thus it makes harder to observe 
the transition at some distance between $R$ scaling and $R^{3 / 2}$, which indicate the increasing effect of scattering relative to diffraction. 

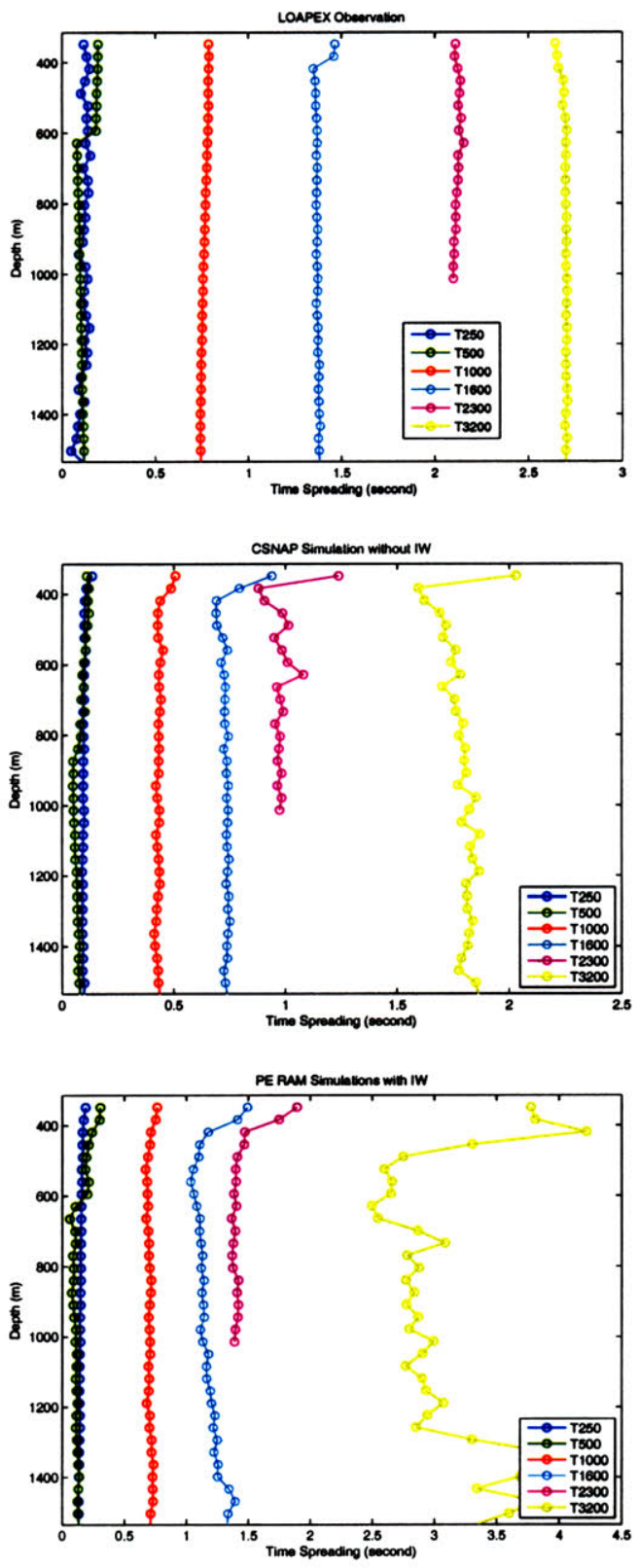

Figure 4-26: The time spreading of mean front intensity in the post-finale region at each LOAPEX stations. The upper panel is the LOAPEX observations. The middle panel is the simulations without internal waves. The lower panel is the simulations with internal waves. Note there are only 20-hydrophone data for the station T2300. 


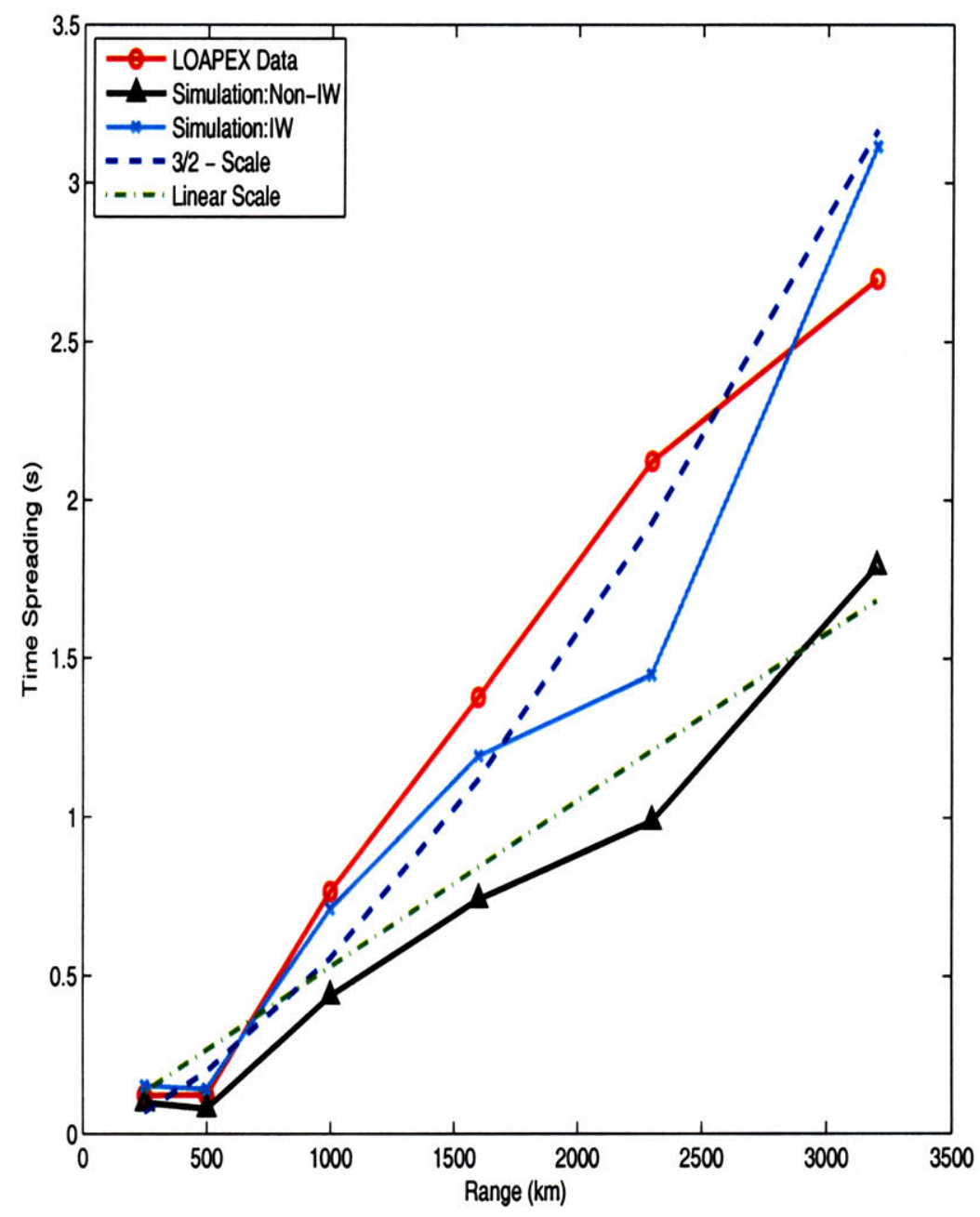

Figure 4-27: The time spreading scales for LOAPEX observation and simulations with/without internal waves. Two different scales (linear and $\frac{3}{2}$ ) are indicated for the comparison. 


\subsection{Summary}

The analysis result of the loapex off-axis sound source transmissions data shows the good quantitative measurement of this shadow zone arrival, which is the high angle sound energy is attracted into low angle region (sound channel depth) due to both diffraction and scattering effect. In this case, off-axis sound source transmission introduce some additional complicating effect, not provided from the on-axis source case. This shadow zone arrivals are mostly concentrated in the horizontal direction (in the temporal scale) after the last ray arrival. For the on-axis sound source transmission, the sound energy is spread out in the vertical direction.

Through the comparison between the numerical simulations (with/without internal wave field) and observation result, surprisingly, we find the they are in good agreement up to range of $1600 \mathrm{~km}$ in the both early arrival region and finale region. But for the longer range (from $2300 \mathrm{~km}$ to $3200 \mathrm{~km}$ ), though the early arrival comparisons are still in satisfactory, there are apparent discrepancy in the finale and post-finale region. It looks like the numerical simulations(with/without $1 / 2 \mathrm{GM}$ internal wave) under-predict the shadow zone arrivals. Two possibilities could make explanations:

1. The signal processing in the LOAPEX data: The signal noise ratio in the loapex data for the stations T2300 and T3200 is quite low in the finale region. There are some further advance techniques: Doppler correction, adaptive beamforming or mode analysis could lead more insight in this analysis.

2. For longer range transmissions, the internal wave scattering is definitely not the only dominant effect for the finale region arrivals. As we know, other meso- or large scale ocean fluctuations might contribute a lot in this case too. But right now, since we only have statistical GM internal wave model, by simply tuning up the GM energy(from $1 / 2$ to one or two) does not explain those discrepancy in the finale region arrival. 


\section{Chapter 5}

\section{Conclusions and Future Directions}

\subsection{Thesis Contributions}

This dissertation has developed some methods for estimating statistical properties of acoustic arrivals transmitted in the random deep ocean scenario. This chapter will summarize the key contributions of the work and indicate directions for future research.

Propagation of sound in a random inhomogeneous medium is described by a wave equation in which the sound velocity is a random function of coordinates and sometimes of time. The solution of this complicated statistical problem can only be obtained by means of approximate methods. At present the most developed of them are the method of small perturbations, the smooth perturbations method (Rytov's method), and the parabolic equation method. The statistics of acoustic fields after propagation through internal-wave-induced sound-speed fluctuations is explored experimentally and theoretically. This thesis is closely related to the series of long-range deep-ocean low-frequency sound propagation experiments in the North Pacific Ocean in the last decade. Away from ocean boundaries, it is generally accepted that internal waves are the dominant source of high frequency fluctuations in ocean acoustic transmissions. Sound waves from a point source are perturbed from simple spherical waves into complicated wave fronts, with random variability of signal phase and amplitude in both time and space. Understanding the principle of sound wave propagating through random medium in the ocean is the key to use acoustic method to monitor deep ocean, measure ocean climate change and global underwater communication. While much theoretical research has been done on long-range propagation in deep ocean, none of the various theories developed over the last two decades successfully predicts received signal 
characteristics as a function of range, frequency, depth, or internal wave intensity.

The first contribution of this thesis is a quantification of space-time scales of ocean sound speed variability contributed from different bands of internal waves continuum, near inertial waves, internal tides and sub-inertial motions(mesoscale) from the North Pacific Acoustic Laboratory(NPAL) 98-99 environmental data. The validity of Garret\&Munk(GM) internal wave model was checked in the upper ocean of eastern North Pacific. All those results impose hard bounds on the strength and characteristic scales of sound speed fluctuations one might expect in the region of the North Pacific for both internal-wave band fluctuations and mesoscale band fluctuations.

The second contribution of this research is a detailed analysis of the low frequency sound arrivals using 6 days of data from a 20-element, $700 \mathrm{~m}$ long vertical receiving array located 87-km distant, and spanning depth region 900-1600-m in the North Pacific Ocean. The observed acoustic variability is compared with acoustic predictions based on the weak fluctuation theory of Rytov, and direct parabolic equation, Monte Carlo simulations. The comparisons show that a resonance condition exists between the local acoustic ray and the internal wave field such that only the internal waves whose crests are parallel to the local ray path will contribute to acoustic scattering: This effect leads to an important filtering of the acoustic spectra relative to the internal wave spectra, such that rays with high grazing angles do not acquire scattering contribution due to low frequency internal waves. We believe that this is the first observational evidence for the acoustic ray and internal wave resonance.

The third contribution of this work is a detailed examination of the evolution, with distance, of the acoustic arrival pattern of the off-axis sound source transmissions in LOAPEX experiment. The observations of mean intensity time-fronts are compared to the deterministic ray, parabolic equation( $\mathrm{PE}$ )(with/without internal waves) and (one-way coupled)normal mode calculations. We found that the observed well-resolved wave front pattern in the early arrival region is reproduced by the the numerical simulation. The diffraction effect is dominant in the shorter range transmission; the high angle energy excited from off-axis sound source are re-distributed into lower angle region. In the longer range, the (internal wave and other random medium effect) scattering effect starts to kick in and smear the energy in both spatial and temporal scales, and eventually has a dominant role in the finale region. 


\subsection{Future Work}

\subsubsection{Data Processing}

In the LOAPEX data processing, a constant Doppler (e.g. constant relative velocity of the source and receiver) was assumed for the entire signal duration. The method of solution was to process for a range of possible Dopplers and to select the output with the maximum value. Apparently, this is not optimum for the LOAPEX acoustic data because of the nonconstant source motion during the lengthy transmissions (up to 80 minutes). Although the method addresses the slow moving receiver array, the method does not separate Doppler caused by the random motion of the medium. One of future work will be incorporate the Doppler Toolbox, developed by Rex Andrew at APL-UW, and the receiving array solutions determined by Frank Henyey and Brad Bell at APL-UW. This new approach should allow us to determine if the differences between the, under-predicted finale-region arrival and the observation are caused by the method of treating the Doppler correction.

The LOAPEX acoustic transmission path had a relatively strong range-dependent sound channel depth, so the very sparse CTD prole casts(only seven) in this wide range of 3200 $\mathrm{km}$ need to be filled in with additional LOAPEX observational data (XBT and UCTD) to provide a more detailed range resolution of the background sound speed field. The lack of resolution may have limited the performance of previous simulations.

\subsubsection{Numerical Modeling: Ocean Modeling and Acoustic Propagation Modeling}

\section{Ocean Modeling}

Internal Wave Simulation In the beginning of this thesis, we started with the assumption that the internal wave field is the dominant source of the sound speed fluctuation in the deep ocean. Through out this work, the GM internal wave ocean model is assumed for both numerical simulation and analytic derivation. We successfully predict the variability of both the intensity and phase of acoustic signals in the range of $87 \mathrm{~km}$ with a low-frequency and broad band sound source. In effect, the assumption of the dominant effect of the internal wave in the deep ocean scenario is verified.

Right now, there are two kinds of statistical internal wave models for numerical simu- 
lations of GM internal waves which are widely used in the deep ocean acoustic community. One is the Colosi \& Brown (1998) IW model, and the other is the Henyey-Wolfson model, developed by Frank Henyey and Michael Wolfson at APL-UW. The basic difference between these two models is the method of finding the internal-wave eigenvalues and modes. Apparently, the UW model performs better and uses an adaption of the techniques used in the acoustic normal mode program Kraken. These techniques are based upon a finite-difference approximation of the applicable differential equation, the application of the Sturm sequence, a bisection to determine the eigenvalues, and an inverse iteration to find the eigenvectors or modes. In the Colosi \& Brown model, a WKB scaling of both the modal amplitudes and the depth coordinate is invoked. So there will be errors for small "j" and high frequencies due to the WKB approximation. So the long-range acoustic propagation simulations conducted with an internal wave model and the parabolic equation method, of the LOAPEX experiment, show that the wavefront pattern in the finale region is very sensitive to the large-scale internal waves, i.e. the small wave number " $\mathrm{j}$ ". That means that the error for small " $\mathrm{j}$ " internal wave simulations might be very important for the simulations of arrivals in the finale region. So the comparison of these two internal wave models should be checked with observation data in the future work.

Non-IW Ocean Process Modeling On the debate of the size of the effect of fine structure in the upper ocean on acoustic fluctuation, the conclusions from my thesis are (for the fine structure only):

1. The internal wave is a dominant source for low frequency acoustic fluctuation in the deep ocean. It is a broadband fluctuation source in both the temporal and spacial scales.

2. The fine structure excists in the upper ocean too, but so far we only have the observation evidence in the spacial scale. The fine structure is not any kind form of wave. It is hard to discover its temporal variability until it is actually measured carefully. Furthermore, it is very possible that the empirical GM spectrum actually contains the fine structure effect.

However, oceanographic observations show that there are several differences between the real ocean sound speed fluctuations and those modeled using the GM spectrum, especially in 
the upper ocean where actually strongest scattering is induced by the upper turning points. Recent SeaSoar measurements and PE simulations using that data ocean "spiceness" temperature and salinity fluctuations that result in no density perturbation may also play a significant role in long-range propagation[29]. The statistical modeling of those non-internal wave effect will also be of interest in future work.

\section{Acoustic Propagation Modeling}

Up to now, the only feasible numerical simulation method with a statistical internal wave field, has been the parabolic equation method. The two most popular parabolic equation codes are RAM and UMPE. So it would be very interesting to compare those two codes to the more accurate one-way coupled normal modes code, like CSNAP with the exact same small-scale perturbed sound-speed field.

\subsubsection{Theory Prediction}

\section{Short Range, Unsaturated Scattering: Weak Fluctuation Theory}

The statistical characteristic of the sound wave field in 20-minute and 40-minute period are presented in this thesis, but it will be very interesting to investigate the phase fluctuation of sound wave field in longer periods. In addition, it would be interesting to explore how the sound wave scattering is evolving along the transmission path, the sound transmission at different ranges will be the key observation for this problem. Actually those observation are made in the NPAL04 experiment, so the data analysis of those acoustic data will be very important work in the future. A further study will be to test this theory for LOAPEX station T50 receptions and then extend this prediction to the LOAPEX T250 stations early arrival, or even station T500s early arrivals. The ultimate goal is examine the limits of validity of Rytov theory at increasing range, higher frequency, and different ray paths.

\section{Long Range, Saturated Scattering: Couple Normal Modes Theory}

The solution of the sound propagating through the real ocean will be different from the simplified case, which is solved by using Rytov method based on the Ray theory. There is validity limitation for the low frequency and long range. For a better understanding acoustic statistics, the developing a statistical couple mode theory will be the one of main 
objectives for future work.

Andrey Morozov (of Woods Hole Oceanographic Institution) and John Colosi (of Naval Postgraduate School) have worked to develop a coupled mode theory based on the work of Dozier and Tappert(1978), and Van Kampen (1992). They modified original approach and adapt the techniques enabling the computation of the important cross mode coherences. This method is able to predict the multi-megameter range evolution of the mean intensity to within a few $\mathrm{dB}$. In future work, one should follow up this approach of computing the cross mode coherence across frequency to predict the broadband arrivals, and then to use it to compare to the observational result at longer-range stations. 


\section{Appendix A}

\section{Maximum-Likelihood Estimate}

The maximum-likelihood estimate of a parameter vector $\alpha$ is usually obtained by maximizing the joint probability density function of a set of observed random variables with respect to $\alpha$. When the observation is a random process rather than a set of random variables, the estimate is obtained by maximizing a function called the likelihood ratio with respect to the unknown parameters. If $n(t)$ in Eq.3.1 is white Gaussian noise with a two-sided spectral density $\frac{1}{2} N_{0}$ then the likelihood ratio for $r(t)$ is

$$
\Lambda\left[r(t),\left\{A_{i}, \tau_{i}\right\}\right]=\exp \left\{\frac{2}{N_{0}} \int_{0}^{T} r(t) \sum_{i=1}^{N} A_{i} s\left(t-\tau_{i}\right) d t-\frac{1}{N_{0}} \int_{0}^{T}\left[\sum_{i=1}^{N} A_{i} s\left(t-\tau_{i}\right)\right]^{2} d t\right\}
$$

where $[0, T]$ is the observation-time interval. The exponetial function is monotonic and

$$
\operatorname{Max}_{\left\{A_{i}, \tau_{i}\right\}} \Lambda\left[r(t),\left\{A_{i}, \tau_{i}\right\}\right]=\operatorname{Max}_{\left\{A_{i}, \tau_{i}\right\}} \ln \Lambda\left[r(t),\left\{A_{i}, \tau_{i}\right\}\right]
$$

The logarithm of the likelihood ratio can be written in matrix notation as

$$
\ln \Lambda\left[r(t),\left\{A_{i}, \tau_{i}\right\}\right]=\left(2 / N_{0}\right) A^{T} \Phi-(1 / N 0) \Lambda^{T} \Lambda A
$$

where

$$
\begin{aligned}
A^{T} & =\left(A_{1}, A_{2}, \ldots, A_{N}\right), \\
\Phi^{T} & =\left(\int_{0}^{T} r(t) s\left(t-\tau_{1}\right) d t, \ldots, \int_{0}^{T} r(t) s\left(t-\tau_{N}\right) d t\right),
\end{aligned}
$$


$\Lambda=\left[\begin{array}{cccc}\lambda_{11} & \lambda_{12} & \cdots & \lambda_{1 N} \\ \ldots \ldots & \ldots & \ldots & \ldots \\ \lambda_{N 1} & \lambda_{N 2} & \cdots & \lambda_{N N}\end{array}\right]$

where

$\lambda_{i j}=\int_{0}^{T} s\left(t-\tau_{i}\right) s\left(t-\tau_{j}\right) d t$

The maximum-likelihood estimates are the values of $A_{i}$ and $\tau$ that maximize Eq.A.3. Maximizing with respect to A yields

$$
\nabla_{A}\left(2 A^{T} \Phi-A^{T} \Lambda A\right)=0
$$

where $\nabla_{A}=\left(\frac{\partial}{\partial A_{1}}, \frac{\partial}{\partial A_{2}}, \ldots, \frac{\partial}{\partial A_{N}}\right)$

Carrying out the differentiation in Eq.A.4 produces the following vector equation which the amplitude vector estimate must satisfy:

$$
A=\Lambda^{-1} \Phi
$$

Substituting Eq.A.5 into Eq.A.3 and maximizing with respect to the set of $\tau_{i}$ yields

$$
\operatorname{Max}_{\left(\tau_{i}\right)}\left[2 \Phi^{T} \Lambda^{-1 T} \Phi-\Phi^{T} \Lambda^{-1} \Phi\right]
$$

However, because $\Lambda$ and $\Lambda^{-1}$ are symmetric, Eq.A.6 can be written as

$$
\operatorname{Max}_{\left(\tau_{i}\right)}\left[\Phi^{T} \Lambda^{-1} \Phi\right]
$$

Therefore, to determine the maximum-likehood estimate, Eq.A.7 must first be maximized with to the set of arrival times. The resulting set of time estimates are then used in Eq.A.5 to obtain amplitude estimates. It should be noted that the maximum-lielihood estimation of the $2 N$ parameters only requires finding the maximum of a function in $\mathrm{N}$-dimensional space. A "brute force" minimum mean-square error estimate would require finding the extremum of a function in $2 N$ dimensional space. 


\section{Appendix B}

\section{Rytov Method}

\section{B.1 Rytov General Solution to Stochastic Wave Equation}

We start from the stochastic Helmholtz Equation:

$$
\nabla^{2} \psi+\frac{\omega^{2}}{c_{0}^{2}(1+\mu(\vec{x}, t))^{2}} \psi=0, \text { with } \mu(\vec{x}, t)=\frac{\delta c(\vec{x}, t)}{c_{0}}<<1
$$

then

$$
\nabla^{2} \psi+q_{0}^{2} \psi-2 q_{0}^{2} \mu(\vec{x}, t) \psi \simeq 0, q_{0}=\frac{\omega}{c_{0}} \text { which is free space wave number }
$$

Note that Eq.B.2 has multiplicative noise because of the $\mu \psi$ term. For Rytov method, $\psi$ can be expanded in the exponential.

$$
\psi=\exp \left(\varphi_{0}+\varphi_{1}+\ldots\right)=\exp (\varphi)
$$

Substituting Eq.B.3 into Eq.B.2 we get the Riccati Equation which is nonlinear:

$$
\nabla^{2} \varphi+(\nabla \varphi)^{2}+q_{0}^{2}(1-2 \mu)=0
$$

To zeroth and first order we get:

$$
\nabla^{2} \varphi_{0}+\left(\nabla \varphi_{0}\right)^{2}+q_{0}^{2}=0
$$




$$
\nabla^{2} \varphi_{1}+\left(\nabla \varphi_{1}\right) \cdot\left(\nabla \varphi_{0}\right)=2 q_{0}^{2} \mu
$$

The first equation is satisfied by the unperturbed solution $\psi_{0}=\exp \left(\varphi_{0}\right)$. To solve the second equation we make the substitution

$$
\begin{gathered}
\varphi_{1}=\exp \left(-\varphi_{0}\right) u \\
\nabla^{2} u+q_{0}^{2} u=2 q_{0}^{2} \mu \exp \left(\varphi_{0}\right)
\end{gathered}
$$

Now notice that we have additive noise term in the right side of equation. This equation can be easily solved using the Green's Function.

$$
\begin{gathered}
u=-2 q_{0}^{2} \int_{V} G\left(\vec{x}-\overrightarrow{x^{\prime}}\right) \mu\left(\overrightarrow{x^{\prime}}\right) \exp \left(\varphi_{0}\left(\overrightarrow{x^{\prime}}\right)\right) \mathrm{d}^{3} \overrightarrow{x^{\prime}}=-2 q_{0}^{2} \int_{V} G\left(\vec{x}-\overrightarrow{x^{\prime}}\right) \mu\left(\overrightarrow{x^{\prime}}\right) \psi_{0}\left(\overrightarrow{x^{\prime}}\right) \mathrm{d}^{3} \overrightarrow{x^{\prime}} \\
G\left(\vec{x}-\overrightarrow{x^{\prime}}\right)=\frac{\exp \left(i q_{0}\left|\vec{x}-\overrightarrow{x^{\prime}}\right|\right)}{4 \pi\left|\vec{x}-\overrightarrow{x^{\prime}}\right|}
\end{gathered}
$$

which is free space Green's function. Using the fact that $\varphi_{0}=\exp \left(\psi_{0}\right)$ and inserting the Green's function, then the final result is

$$
\varphi_{1}(\vec{x})=\exp \left(-\varphi_{0}\right) u=-\frac{q_{0}^{2}}{2 \pi} \int_{\mathbf{V}} \frac{\exp \left(i q_{0}\left|\vec{x}-\overrightarrow{x^{\prime}}\right|\right)}{\left|\vec{x}-\overrightarrow{x^{\prime}}\right|} \mu\left(\overrightarrow{x^{\prime}}\right) \frac{\psi_{0}\left(\overrightarrow{x^{\prime}}\right)}{\psi_{0}(\vec{x})} \mathrm{d}^{3} \overrightarrow{x^{\prime}}
$$

This is the basic equation for propagation in a random medium using the Rytov Approximation.

\section{B.2 Rytov Theory for Incident Plane Wave and Small Angle Propagation}

For the plane wave propagation in the $x$-direction, i.e. assume $\psi_{0}(\vec{x})=\exp \left(i q_{0} x\right)$, the Rytov theory (Eq.B.11) gives

$$
\varphi_{1}(\vec{x})=\exp \left(-\varphi_{0}\right) u=-\frac{q_{0}^{2}}{2 \pi} \int_{\mathbf{V}} \frac{\exp \left(i q_{0}\left|\vec{x}-\overrightarrow{x^{\prime}}\right|\right)}{\left.\mid \vec{x}-\overrightarrow{(} x^{\prime}\right) \mid} \mu\left(\overrightarrow{x^{\prime}}\right) \exp \left(i q_{0}\left(x^{\prime}-x\right)\right) d^{3} \overrightarrow{x^{\prime}}
$$

Now lets assume that the observation plane is located at range $\mathrm{R}$ or

$$
\vec{x}=(R, y, z)
$$


Consider the maximum scattering angle (by diffraction theory) which is given by

$$
\theta_{\max } \sim \frac{\lambda}{l_{0}}, \lambda=\text { wavelength, } l_{0}=\text { smallest scale of fluctuations of } \mu
$$

If small angle scattering is assumed $\left(\theta_{\max } \ll 1\right.$, essentially the parabolic approximation) then

$$
\left|\vec{x}-\overrightarrow{x^{\prime}}\right|=\left[\left(R-x^{\prime}\right)^{2}+\left(y-y^{\prime}\right)^{2}+\left(z-z^{\prime}\right)^{2}\right]^{1 / 2} \simeq\left(R-x^{\prime}\right)\left[1+\frac{1}{2} \frac{\left(y-y^{\prime}\right)^{2}+\left(z-z^{\prime}\right)^{2}}{R-x^{\prime}}\right]
$$

Substituting back into Equation of Rytov function:Eq.B.12, and get

$$
\varphi_{1}(\vec{x})=-\frac{q_{0}^{2}}{2 \pi} \int_{0}^{R} d x^{\prime} \int_{-\infty}^{\infty} d y^{\prime} \int_{-\infty}^{\infty} d z^{\prime} \frac{\mu\left(\overrightarrow{x^{\prime}}\right)}{R-x^{\prime}} \exp \left[i q_{0}\left(\frac{\left(y-y^{\prime}\right)^{2}+\left(z-z^{\prime}\right)^{2}}{2\left(R-x^{\prime}\right)}\right)\right]
$$

Note that $\frac{1}{\left|\vec{x}-\vec{x}^{\prime}\right|} \sim \frac{1}{\left(R-x^{\prime}\right)}$ is used. The volume integral has been contracted to the region $0<x^{\prime}<R$. This is justified because we are looking at small angle forward scattering. This is the Rytov Result for an incident plane wave and small angle scattering.

$\psi$ in Eq.B.3 is complex function, so it can be written as:

$$
\psi=\psi_{0} \exp \left(\varphi_{1}\right)=A \exp (\mathrm{i} \phi)
$$

So,from Eq.B.17

$$
\varphi_{1}=\ln \left(\frac{\psi}{\psi_{0}}\right)=\ln \left(\frac{A \exp (i \phi)}{A_{0} \exp \left(i \phi_{0}\right)}\right)=\ln \left(\frac{A}{A_{0}}\right)+i\left(\phi-\phi_{0}\right)
$$

So the log-amplitude $\chi$ and phase $\phi$ are defined as:

$$
\begin{gathered}
\chi=\frac{1}{2}\left(\varphi_{1}+\varphi_{1}^{*}\right)=\ln \left(\frac{A}{A_{0}}\right) \equiv \log \text {-amplitude fluctuation } \\
\phi=\frac{1}{2 i}\left(\varphi_{1}-\varphi_{1}^{*}\right)=\left(\phi-\phi_{0}\right)=\phi_{1} \equiv \text { phase fluctuation }
\end{gathered}
$$




\section{B.3 Spectra of Phase and log-amplitude: No waveguide}

The well known result for the spectrum of phase $\phi$ and log-amplitude $\chi$ for a point source in the Rytov approximation (Ishimaru,1977, and Munk and Zachariasen 1976) is

$$
F_{\phi, \chi}\left(R, k_{y}, k_{z}\right)=\pi k_{0}^{2} R \int_{0}^{1} \Phi\left(0, k_{y} / s, k_{z} / s\right)\left[1 \pm \cos \left(\frac{\left(k_{y}^{2}+k_{z}^{2}\right) R_{f}^{2}(x)}{2 \pi s^{2}}\right)\right] \mathrm{d} s
$$

where $s=x / R$ is the normalized range, $R_{f}^{2}(x)=\lambda x(R-x) / R=\lambda R s(1-s)$ is the Fresnel zone, and $k_{0}=\omega / c_{0}=2 \pi / \lambda$ is the acoustic wavenumber. The Garrett-Munk(GM) 3-D spectrum of relative sound speed fluctuations is:

$$
\Phi\left(k_{x}, k_{y}, k_{z}\right)=\mu_{0}^{2} \frac{k_{z^{*}}}{\pi} \frac{1}{\left(k_{z}^{2}+k_{z^{*}}^{2}\right)} \frac{2}{\pi^{2}} \frac{\left|\hat{k_{z}}\right| \sqrt{k_{x}^{2}+k_{y}^{2}}}{\left(k_{x}^{2}+k_{y}^{2}+\hat{k}_{z}^{2}\right)^{2}}
$$

where $k_{z^{*}}=\pi j_{*} / D, D$ is the water depth, and $\hat{k_{z}}=k_{z}\left(f / N_{0}\right)$. Here $k_{z}$ is a continuous variable defined on the interval $-\infty$ to $\infty$, and the normalization condition is $\mu_{0}^{2}=$ $\int_{-\infty}^{\infty} d \mathbf{K} \Phi(\mathbf{K})$. An alternative representation in terms of the mode number $j,(1 \leq j \leq \infty)$, is

$$
\Phi\left(k_{x}, k_{y}, k_{z}(j)\right)=\mu_{0}^{2} \frac{\pi^{2}}{D^{2} M} \frac{1}{\left(k_{z}^{2}+k_{z^{*}}^{2}\right)} \frac{2}{\pi^{2}} \frac{\left|\hat{k_{z}}\right| \sqrt{k_{x}^{2}+k_{y}^{2}}}{\left(k_{x}^{2}+k_{y}^{2}+\hat{k}_{z}^{2}\right)^{2}}
$$

where $M=\sum_{j=1}^{\infty}\left(j^{2}+j_{*}^{2}\right)^{-1}, k_{z}=\pi j / D$, and $\hat{k_{z}}=k_{z}\left(f / N_{0}\right)$, and the normalization condition is $\mu_{0}^{2}=\sum_{j=1}^{\infty} \int_{-\infty}^{\infty} d k_{x} d k_{y} \Phi\left(k_{x}, k_{y}, k_{z}(j)\right)$. The WKB dispersion relation typically used with the GM spectrum is:

$$
\omega^{2}=N_{0}^{2} \frac{k_{x}^{2}+k_{y}^{2}}{k_{z}^{2}}+f^{2}
$$

, First we change variables from $k_{y}$ to $\omega$ utilizing the fact that $k_{x}=0$,

$$
F_{\phi, \chi}\left(R, \omega, k_{z} / s\right)=F_{\phi, \chi}\left(R, k_{y} / s, k_{z} / s\right) \frac{1}{s} \frac{d k_{y}}{d \omega}
$$

Using

$$
k_{y} \frac{d k_{y}}{d \omega}=\frac{\omega k_{z}^{2}}{N_{0}^{2}}, k_{y}=\left(\omega^{2}-f^{2}\right)^{(1 / 2)} \frac{k_{z}}{N_{0}}
$$




$$
\begin{gathered}
\Phi\left(0, \omega, k_{z}\right)=\frac{\mu_{0}^{2}}{s} \frac{2 k_{z^{*}} f^{2}}{\pi^{3}} \frac{\omega^{-3}}{\hat{k_{z}\left(k_{z}^{2}+k_{z^{*}}^{2}\right)}, \omega \geq f} \\
\Phi\left(0, \omega, k_{z}(j)\right)=\frac{\mu_{0}^{2}}{s} \frac{f^{2}}{D^{2} M} \frac{\omega^{-3}}{\hat{k}_{z}\left(k_{z}^{2}+k_{z^{*}}^{2}\right)}, \omega \geq f
\end{gathered}
$$

the frequency-wavenumber spectrum becomes,

$$
F_{\phi, \chi}\left(R, \omega, k_{z}\right)=\pi q_{0}^{2} R \int_{0}^{1} \Phi\left(0, \omega, k_{z} / s\right)\left[1 \pm \cos \left(\gamma(s)\left(1+\frac{\omega^{2}}{N_{0}^{2}}\right)-\alpha(s)\right)\right] d s
$$

where $\gamma(s)=k_{z}^{2} R_{f}^{2}(s) /\left(2 \pi s^{2}\right)$, and $\alpha(s)={\hat{k_{z}}}^{2} R_{f}^{2}(s) /\left(2 \pi s^{2}\right)$.

The variances of log-amplitude and phase are obtained by integrating (or summing) over frequency and wavenumber,

$$
\left\langle\phi^{2}\right\rangle,\left\langle\chi^{2}\right\rangle=\int_{-\infty}^{\infty} d k_{z} \int_{f}^{\infty} d \omega F_{\phi, \chi}\left(R, \omega, k_{z}\right) \text { or } \sum_{j=1}^{\infty} \int_{f}^{\infty} d \omega F_{\phi, \chi}\left(R, \omega, k_{z}(j)\right)
$$

\section{B.4 Spectra of Phase and log-amplitude: Waveguide}

The waveguide case has been treated by Munk and Zachariasen(1976) with the result that the homogeneous condition is simply applied locally along the unperturbed ray path $z_{r}(x)$, and the stretching effect of $s$ is ignored (i.e. the wave acts locally like a plane wave and $s \simeq 1$ ). Thus the point source spectra for propagation along a ray path become,

$$
F_{\phi, \chi}\left(R, k_{y}, k_{z}\right)=\pi k_{0}^{2} \int_{\Gamma} d x \sec ^{2} \theta \Phi\left(0, k_{\perp}\left(k_{y} / s, k_{z} ; x\right) ; z(x)\right)\left[1 \pm \cos \left(\frac{k_{y}^{2} R_{f y}^{2}(x)}{2 \pi s^{2}}+\frac{k_{z}^{2} R_{f z}^{2}(x)}{2 \pi}\right)\right]
$$

where it is understood that the integrand is not changing rapidly with range. Here we have,

$$
\begin{gathered}
R_{f y}^{2}(x)=\frac{\lambda x(R-x)}{R} \\
k_{\perp}\left(z_{r}(x)\right)=\left(-k_{z} \tan \theta\left(z_{r}(x)\right), k_{y}, k_{z}\right) \\
k_{h}=\left(k_{z}^{2} \tan ^{2} \theta+k_{y}^{2}\right)^{1 / 2} \sim \frac{\pi j}{N_{0} B}\left(\omega^{2}-f^{2}\right)
\end{gathered}
$$




$$
\begin{gathered}
k_{y}=\frac{\pi j}{N_{0} B}\left(\omega^{2}-\omega_{L}^{2}\right)^{1 / 2}, \omega_{L}=f^{2}+N^{2} \tan ^{2} \theta \\
k_{z}=\frac{\pi j N(z)}{N_{0} B}, N_{0} B=\int_{0}^{D} N(z) d z
\end{gathered}
$$

$R_{f z}^{2}$ is the vertical Fresnel zone computed from Green's function.

The 3-D spectrum of relative sound speed fluctuations is:

$$
\Phi\left(k_{x}, k_{y}, k_{z}\right)=\mu_{0}^{2} \frac{k_{z^{*}}}{\pi} \frac{1}{\left(k_{z}^{2}+k_{z^{*}}^{2}\right)} \frac{2}{\pi^{2}} \frac{\left|\hat{k_{z}}\right| \sqrt{k_{x}^{2}+k_{y}^{2}}}{\left(k_{x}^{2}+k_{y}^{2}+\hat{k}_{z}^{2}\right)^{2}}
$$

where $k_{z^{*}}=\pi j_{*} / D, D$ is the water depth, and $\hat{k_{z}}=k_{z}\left(f / N_{0}\right)$. As before we change variables from $k_{y}$ to $\omega$ using,

$$
F_{\phi, \chi}\left(R, \omega, k_{z}\right)=F_{\phi, \chi}\left(R, k_{y}, k_{z}\right) \frac{d k_{y}}{d \omega}
$$

This transformation is accomplished using Eqs.B.33-B.34 with

$$
k_{y} \frac{d k_{y}}{d \omega}=\frac{\omega k_{z}^{2}}{N^{2}}
$$

we obtain

$\Phi\left(0, k_{\perp}\left(\omega, k_{z}(j)\right) ; z\right)=\Phi\left(0, k_{\perp}\left(k_{y}, k_{z}\right) ; z\right) \frac{d k_{y}}{d \omega}=\frac{\mu_{0}^{2} N^{3}}{N_{0}^{3}} \frac{2 f^{2} N^{2}}{\left(N_{0} B\right)^{2} M} \frac{\omega^{-3}}{\hat{k}_{z}\left(k_{z}^{2}+k_{z^{*}}^{2}\right)}\left(\frac{\omega^{2}-f^{2}}{\omega^{2}-\omega_{L}^{2}}\right)^{1 / 2}, \omega>\omega_{L}$

and the frequency-wavenumber spectrum becomes,

$F_{\phi, \chi}\left(R, \omega, k_{z}(j)\right)=\pi k_{0}^{2} \int_{\Gamma} d x \sec ^{2} \theta \Phi\left(0, \omega, k_{z}(j) ; z\right)\left[1 \pm \cos \left(\frac{k_{z}^{2} \frac{\left(\omega^{2}-\omega_{L}^{2}\right)}{N^{2} s^{2}} R_{f y}^{2}(x)+k_{z}^{2} R_{f z}^{2}(x)}{2 \pi}\right)\right]$

For short ranges like those considered in this paper the vertical Fresnel zone is much larger than the transverse Fresnel zone, thus we make the approximation

$$
F_{\phi, \chi}\left(R, \omega, k_{z}(j)\right) \simeq \pi k_{0}^{2} \int_{\Gamma} d x \sec ^{2} \theta \Phi\left(0, \omega, k_{z}(j) ; z\right)\left[1 \pm \cos \left(\frac{k_{z}^{2} R_{f z}^{2}(x)}{2 \pi}\right)\right]
$$

Eq.B.42 should be compared with Eq.121 from Munk and Zachariasen. Eq.B.42 says that for each frequency $\omega$ and each vertical wavenumber $k_{z}$ we integrate the spectrum along the ray. However, there are forbidden regions in this integral, specially where Eq.B.35 cannot 
be satisfied at low frequency, and where $\omega>N(z)$. Thus to specially indicate the forbidden regions we add the Heavyside step functions to the spectrum giving,

$$
F_{\phi, \chi}\left(R, \omega, k_{z}(j)\right)=\pi k_{0}^{2} \int_{\Gamma} d x \sec ^{2} \theta \Phi\left(0, \omega, k_{z}(j) ; z\right)\left[1 \pm \cos \left(\frac{k_{z}^{2} R_{f z}^{2}(x)}{2 \pi}\right)\right] H\left[\omega-\omega_{L}\left(z_{r}(x)\right)\right] H\left[N\left(z_{r}(x)\right)-\omega\right]
$$




\section{Appendix C}

\section{Computation of the Fresnel Zone}

In the parabolic approximation the ray equation is

$$
\partial_{x x} z_{r}+\partial_{z} U\left(z_{r}\right)=0
$$

where the sound speed profile has the form $c(z)=c_{0}(1+U(z))$. Examining small vertical deviations from a ray such that $z(x)=z_{r}(x)+\zeta(x)$, we plug this expression into Eq. C.1 and linearize to obtain the "ray-tube" equation

$$
\partial_{x x} \zeta+\zeta \partial_{z z} U\left(z_{r}\right)=0
$$

Importantly the physical interpretation of the ray-tube function $\zeta$ is

1. that the acoustic ray amplitude is proportional to $\zeta^{-1 / 2}$ and

2. where $\zeta=0$ there is a caustic.

Since Eq. C.2 is second order there are two solutions $\zeta_{1}(x)$ and $\zeta_{2}(x)$, so using the initial conditions

$$
\zeta_{1}(0)=0, \zeta_{1}^{\prime}=1, \zeta_{2}(0)=1, \zeta_{2}^{\prime}(0)=0
$$

the Fresnel zone along the ray path $z_{r}(x)$ can be written (Flatte 1983)

$$
R_{f z}^{2}(x)=\lambda \frac{\zeta_{1}(x)}{\zeta_{1}(R)}\left[\zeta_{2}(x) \zeta_{1}(R)-\zeta_{1}(x) \zeta_{2}(R)\right]
$$

where $\lambda$ is the acoustic wavelength, and $R$ is the range of the receiver. Clearly these 
expressions break down if the receiver is near a caustic(i.e. $\zeta(R)=0$ ). 


\section{Appendix D}

\section{Parabolic Equation Method}

\section{D.1 Derivation of Standard Parabolic Equation}

The starting point is the Helmholtz equation for a constant-density medium in cylindrical coordinates $(r, \varphi, z)$ and for a harmonic point source of time depdence exp $(-i \omega t)$,

$$
\frac{\partial^{2} p}{\partial r^{2}}+\frac{1}{r} \frac{\partial p}{\partial r}+\frac{\partial^{2} p}{\partial z^{2}}+k_{0}^{2} n^{2} p=0
$$

where we have assumed azimuthal symmetry and hence no dependence on the $\varphi$-coordinate. Here $p(r, z)$ is the acoustic pressure, $k_{0}=\omega / c_{0}$ is a reference wavenumber, and $n(r, z)=$ $c_{0} / c(r, z)$ is the index of refraction.

Assuming the solution of Eq.D.1 to take the form

$$
p(r, z)=\psi(r, z) H_{0}^{(1)}\left(k_{0} r\right)
$$

which is an outgoing cylindrical wave solution. The envelope function $\psi(r, z)$ is assumed to be slowly varying in range.

The Hankel function, which satisfied the Bessel differential equation

$$
\frac{\partial^{2} H_{0}^{(1)}\left(k_{0} r\right)}{\partial r^{2}}+\frac{1}{r} \frac{\partial H_{0}^{(1)}\left(k_{0} r\right)}{\partial r}+k_{0}^{2} H_{0}^{(1)}\left(k_{0} r\right)=0
$$


is generally replaced by its asymptotic form for $k_{0} r \gg 1$,

$$
H_{0}^{(1)}\left(k_{0} r\right) \simeq \sqrt{\frac{2}{\pi k_{0} r}} e^{i\left(k_{0} r-\frac{\pi}{4}\right)}
$$

Substituting the trial solution, Eq.(D.2), into the Helmholtz equation(D.1), and making use of the Hankel-function property given by Eq.(D.3), we obtain

$$
\frac{\partial^{2} \psi}{\partial r^{2}}+\left(\frac{2}{H_{0}^{2}\left(k_{0} r\right)} \frac{\partial H_{0}^{(1)}\left(k_{0} r\right)}{\partial r}+\frac{1}{r}\right) \frac{\partial \psi}{\partial r}+\frac{\partial^{2} \psi}{\partial z^{2}}+k_{0}^{2}\left(n^{2}-1\right) \psi=0
$$

Then we make the farfield assumption, $k_{0} r \gg 1$, and use Eq.(D.4) to obtain the simplified elliptic wave equation

$$
\frac{\partial^{2} \psi}{\partial r^{2}}+2 i k_{0} \frac{\partial \psi}{\partial r}+\frac{\partial^{2} \psi}{\partial z^{2}}+k_{0}^{2}\left(n^{2}-1\right) \psi=0
$$

Finally the crucial paraxial approximation is introduced in order to get the standard parabolic wave equation. This small-angle approximation is expressed by

$$
\frac{\partial^{2} \psi}{\partial r^{2}} \ll 2 i k_{0} \frac{\partial \psi}{\partial r}
$$

This paraxial approximation is justified by noting that: the main radial dependence of the field is contained in the Hankel function through the term $\exp \left(i k_{0} r\right)$, while the envelope $\psi$ will vary slowly with range over a wavelength $\lambda$, i.e $\partial \psi / \partial r \ll \psi / \lambda \sim i k_{0} \psi$. By making use of the paraxial approximation in Eq.(D.6), the standard parabolic equation introduced by Hardin and Tappert is

$$
2 i k_{0} \frac{\partial \psi}{\partial r}+\frac{\partial^{2} \psi}{\partial z^{2}}+k_{0}^{2}\left(n^{2}-1\right) \psi=0
$$

\section{D.2 The Split-Step Fourier Algorithm}

The standard parabolic equation can be solved by Fourier transform techniques proposed by Hardin ann Tappert.

First, under assumption that the refraction index $n$ is constant, we start by transforming the entire parabolic equation Eq.(D.8) into $k_{z}$ wave number domain by using Fourier 
transform and its property as:

$$
\begin{gathered}
\psi(r, z)=\int_{-\infty}^{\infty} \psi\left(r, k_{z}\right) e^{i k_{z} z} d k_{z} \\
\psi\left(r, k_{z}\right)=\frac{1}{2 \pi} \int_{-\infty}^{\infty} \psi(r, z) e^{-i k_{z} z} d z \\
\int_{-\infty}^{\infty} \frac{\partial^{2} \psi(r, z)}{\partial z^{2}} e^{-i k_{z} z} d z=-k_{z}^{2} \psi\left(r, k_{z}\right)
\end{gathered}
$$

where $k_{z}$ is the vertical wavenumber. Then the transformed wave equation in $\psi\left(r, k_{z}\right)$ takes the form

$$
2 i k_{0} \frac{\partial \psi}{\partial r}-k_{z}^{2} \psi+k_{0}^{2}\left(n^{2}-1\right) \psi=0
$$

by arranging the terms

$$
\frac{\partial \psi}{\partial r}+\frac{k_{0}^{2}\left(n^{2}-1\right)-k_{z}^{2}}{2 i k_{0}} \psi=0
$$

This is a linear, first-order differential equation with the solution

$$
\psi\left(r, k_{z}\right)=\psi\left(r_{0}, k_{z}\right) e^{-\frac{k_{0}^{2}\left(n^{2}-1\right)-k_{z}^{2}}{2 i k_{0}}\left(r-r_{0}\right)}
$$

Then transform back to the $z$-domain and get the field solution

$$
\psi(r, z)=e^{\frac{i k_{0}}{2}}\left(n^{2}-1\right)\left(r-r_{0}\right) \int_{-\infty}^{\infty} \psi\left(r_{0}, k_{z}\right) e^{-\frac{i\left(r-r_{0}\right)}{2 k_{0}} k_{z}^{2}} e^{i k_{z} z} d k_{z}
$$

Denote the range increment $r-r_{0}$ by $\Delta r$ and denote the Fourier transform from the $z$-domain to the $k_{z}$-domain with the symbol $\mathcal{F}$ and inverse transform with $\mathcal{F}^{-1}$. The field solution can then be written in the compact form

$$
\psi(r, z)=e^{\frac{i k_{0}}{2}\left[n^{2}\left(r_{0}, z\right)-1\right] \Delta r} \mathcal{F}^{-1}\left\{e^{-\frac{i \Delta r}{2 k_{0}} k_{z}^{2}} \mathcal{F}\left\{\psi\left(r_{0}, z\right)\right\}\right\}
$$

which is the split-step marching algorithm proposed by Hardin and Tappert for solving the standard parabolic equation. The solution was derived for the trivial case of a uniform medium, but the error incurred for $n=n(r, z)$ is of order $(\Delta r)^{2}$ and hence can be made arbitrarily small by choosing a small computational range step. The algorithm was shown by Tapper to be unconditionally stable. 


\section{Appendix E}

\section{Vertical Line Array (VLA)}

\section{Calibration}

Based on the information from NPAL04 SPICE cruise plan and report by Worcester, the calibration of VLA is summarized as following. Basically, to get the absolute pressure unit from VLA, the system sensitivity and the signal processing gain are needed.

\section{E.1 System Sensitivity}

The whole system sensitivity is a function of hydrophone sensitivity, system gain in the passband, and Analog to Digital Converter (ADC) least count.

For NPAL04 VLA system, the parameters are list as following:

1. High Tech Inc. ATOC hydrophone sensitivity: $-168 \mathrm{~dB}$ re $1 \mathrm{~V} / \mu \mathrm{Pa}$.

2. AVATOC system gain in the passband:

(a) Input differntial amplifier: $+20 \mathrm{~dB}$ (Hardware selectable to $0,+20,+40 \mathrm{~dB}$ ).

(b) Low pass filter gain (unity): $0 \mathrm{~dB}$.

(c) Programmable gain amplifier (12 or $24 \mathrm{~dB}$ ): $+12 \mathrm{~dB}$ (NPAL 04).

So the system sensitivity at $\mathrm{ADC}$ input is: $-136 \mathrm{dBV} / \mu \mathrm{Pa}(158.5 \mathrm{nV} / \mu \mathrm{Pa})$.

3. ADC Least Count: $-76.3 \mathrm{dBV} /$ count ( $152.6 \mu \mathrm{V} /$ count). The ADC full scale is actually $+/-4.5 \mathrm{~V}$, but the buffer amps driving the ADC's are scaled to make the effective 
range $+/-5 \mathrm{~V}$, and it is 16 -bit $\mathrm{ADC}$. So the $\mathrm{ADC}$ least count is :

$$
20 * \log 10\left(10 /\left(2^{16}\right)\right)=-76.33 \mathrm{dBV} / \text { count }
$$

The whole system sensitivity is :

ADC Least Count - Hydrophone sensitivity - AVATOC system gain, i.e.

$$
-76.33+168-32=59.67 \mathrm{~dB} \text { re } 1 \mu \mathrm{Pa} / \text { count or }(962.72 \mu \mathrm{Pa} / \text { count }) \text {. }
$$

\section{E.2 Signal Processing Gain}

Depends on the different signal transmitted, and the signal length recorded and processed, the different signal processing gain need to be removed to get absolute pressure unit . Generally, there are two steps involved in signal processing to obtain the extra gain on signal to noise ratio:

1. Pulse compression gain: The signal being used is M-sequence with 1023 digits, which introduces $10 * \log 10(1023)=30.1 \mathrm{~dB}$ gain.

2. Period averaging gain: It depends on how many periods being used when the pulse compression is carried out. In this thesis, in order to implement the doppler correction for each period, so the signal is processed for only one period, which means there is no period averaging gain in this process. 


\section{Appendix F}

\section{Ray Dynamics : Travel Time Statistics and Time Spreading Scale}

\section{F.1 Travel Time Statistics}

In ray dynamics, the acoustic travel time can be expressed as a line integral over a Lagrangian density function $L$, which depends on both the ray position $z(x)$, the ray slope $\dot{z}=d z / d x$, and the location along the path $x$ and thus,

$$
T(\text { path })=q_{0} \int_{\Gamma} L(\dot{z}, z ; x) d x
$$

where

$$
\begin{aligned}
L(\dot{z}, z ; x) & =\frac{\dot{z}^{2}}{2}-U(z, x), \\
U(z, x) & =\frac{c(z, x)}{c_{0}}-1, U<<1
\end{aligned}
$$

So travel time fluctuations are associated with the Lagrangian function. Assuming in one dimensional case, the Lagrangian system can be changed to the Hamiltonian system with a change of coordinates $(z, z ; x)$ to $(z, p ; x)$ with

$$
p_{z}=\frac{\partial L(z, \dot{z} ; x)}{\partial \dot{z}}, \text { and } H\left(z, p_{z} ; x\right)=\dot{z} p_{z}-L(z, \dot{z} ; x)
$$


i.e.

$$
\begin{aligned}
& L=p_{z} \frac{d z}{d x}-H, \rightarrow I \frac{d \theta}{d x}-H(I)=I \omega(I)-H(I) \\
& I=\frac{1}{\pi} \int_{z^{-}}^{z^{+}} d z p(H, z)=\frac{1}{\pi} \int_{z^{-}} z^{+} d z\left(c^{-2}-H^{2}\right)^{1 / 2}
\end{aligned}
$$

For the Helmholtz Equation,

$$
H=-\left(c^{-2}-p_{z}^{2}\right)^{1 / 2}, p_{z}=\sin \theta_{r a y} / c .
$$

For $c=c(z)$,

$$
H=-\frac{\cos \theta_{\text {ray }}}{c}=\text { const }=\frac{1}{c\left(z^{-}\right)}=\frac{1}{c\left(z^{+}\right)}=\frac{1}{\hat{c}}
$$

Thus there is important result.

$$
I=\frac{1}{\pi} \int_{z^{-}}^{z^{+}} d z\left(c^{-2}-\hat{c}^{-2}\right)^{1 / 2}, \rightarrow \frac{d I}{d H}=\frac{-1}{2 \pi} R(I)
$$

From the Lagrangian, an expression for the travel time is

$$
\frac{d T}{d x}=\frac{d T}{d \theta} \frac{d \theta}{d x}=\frac{d T}{d \theta} \omega(I)=L=I \omega(I)-H(I)
$$

Integrating over one cycle:

$$
\begin{aligned}
& T(I)=2 \pi\left(I-\frac{H(I)}{\omega(I)}\right) \\
& T(I)=2 \pi I-H(I) R(I)
\end{aligned}
$$

\section{F.1.1 Upper Turning Point Model}

Now lets imagine a scattering event that instantaneously changes the ray trajectory into another trajectory at the upper turning point. Thus the ray changes from one "rangeindependent" tracjectory to another. Lets expand about some reference trajectory $H_{0}\left(I_{0}\right)$, and using Eq. F.9

$$
\begin{aligned}
R\left(H_{0}+\delta H\right) & =R\left(H_{0}\right)+\left.\delta H \frac{\partial R}{\partial H}\right|_{H_{0}}+\left.\frac{\delta H^{2}}{2} \frac{\partial^{2} R}{\partial H^{2}}\right|_{H_{0}}+\ldots \ldots \\
\delta R & =-\left.2 \pi \delta H \frac{\partial^{2} I}{\partial H^{2}}\right|_{H_{0}}-\left.2 \pi \frac{\delta H^{2}}{2} \frac{\partial^{3} I}{\partial H^{3}}\right|_{H_{0}}
\end{aligned}
$$


and also for the travel time and using Eqs. F.9 and F.11 we obtain

$$
\begin{aligned}
T\left(H_{0}+\delta H\right) & =T\left(H_{0}\right)+\left.\delta H \frac{\partial T}{\partial H}\right|_{H_{0}}+\left.\frac{\delta H^{2}}{2} \frac{\partial^{2} T}{\partial H^{2}}\right|_{H_{0}}+\ldots . \\
\delta T & =\left[\left.2 \pi \frac{\partial I}{\partial H}\right|_{H_{0}}-R\left(H_{0}\right)-\left.H \frac{\partial R}{\partial H}\right|_{H_{0}}\right] \delta H-\left[\left.\frac{\partial^{3} I}{\partial H^{3}} H\right|_{H_{0}}+\left.\frac{\partial^{2} I}{\partial H^{2}}\right|_{H_{0}}\right] \frac{\delta H^{2}}{2} \\
& =H_{0} \frac{\delta R}{2 \pi}-\left.\frac{\partial^{2} I}{\partial H^{2}}\right|_{H_{0}} \frac{\delta H^{2}}{2}
\end{aligned}
$$

For $N$ loops we get

$$
\begin{aligned}
\delta \tau & =\sum_{j=1}^{N} \delta \tau_{j}=\sum_{j=1}^{N} H_{0} \frac{\delta R_{j}}{2 \pi}-\left.\frac{\partial^{2} I}{\partial H^{2}}\right|_{H_{0}} \frac{\delta H_{j}^{2}}{2} \\
& =-\left.\frac{\partial^{2} I}{\partial H^{2}}\right|_{H_{0}} \sum_{j=1}^{N} \frac{\delta H_{j}^{2}}{2} \\
\langle\delta \tau\rangle & =-\left.N \frac{\partial^{2} I}{\partial H^{2}}\right|_{H_{0}} \frac{\left\langle\delta H^{2}\right\rangle}{2}
\end{aligned}
$$

because $\delta r=\sum_{j=1}^{N} \delta R_{j}=0$ (eigenray condition). But,

$$
\begin{aligned}
\frac{\partial^{2} I}{\partial H^{2}} & =-\frac{1}{2 \pi} \frac{\partial R}{\partial H}=-\frac{1}{2 \pi} \frac{\partial R}{\partial I} \frac{\partial I}{\partial H}=-\frac{\omega^{\prime}}{\omega} \\
\langle\delta \tau\rangle & =-\left.N \frac{\omega^{\prime}}{\omega}\right|_{I_{0}} \frac{\left\langle\delta H^{2}\right\rangle}{2}
\end{aligned}
$$

where $\omega(I)$ is the ray lope frequency and $\omega^{\prime}=d \omega / d I$.

So the bias depends on $\omega^{\prime}$ and scales like $R^{2}$ because $\left\langle\delta H^{2}\right\rangle$ scales like $\mathrm{R}$.

Similar for the time spread

$$
\left\langle\delta \tau^{2}\right\rangle-\langle\delta \tau\rangle^{2}=N\left(\left.\frac{\omega^{\prime}}{\omega}\right|_{I_{0}}\right)^{2} \frac{\delta H^{4}}{4}
$$

which grows like $R^{3}$. 


\section{Bibliography}

[1] A. Baggeroer and W. Munk. The Heard Island Feasibility Test. Physics Today, 45(9):22-30, 1992.

[2] A. B. Baggeroer, T. G. Birdsall, C. Clark, J. A. Colosi, B. D. Cornuelle, D. Costa, B. D. Dushaw, M. Dzieciuch, A. M. G. Forbes, C. Hill, B. M. Howe, J. Marshall, D. Menemenlis, J. A. Mercer, K. Metzger, W. Munk, R. C. Spindel, D. Stammer, P. F. Worcester, C. Wunsch, and Atoc and Consortium. Ocean climate change: Comparison of acoustic tomography, satellite altimetry, and modeling. Science, 281(5381):1327$1332,1998$.

[3] D. Behringer, T. Birdsall, M. Brown, B. Cornuelle, R. Heinmiller, R. Knox, K. Metzger, W. Munk, J. Spiesberger, R. Spindel, D. Webb, P. Worcester, and C. Wunsch. A demonstration of ocean acoustic tomography. Nature, 299(5879):121-125, 1982.

[4] T. H. Bell. Processing vertical internal wave spectra. Journal of Physical Oceanography, 4(4):669-670, 1974.

[5] J.B. Bowlin, J.L. Spiesberger, T.F. Duda, and L.F. Freitag. Ocean Acoustical Raytracing Software RAY. Tech. Rep. WHOI-93-10, Woods Hole Oceanographic institution, Woods Hole, Mass., 1992. 49pp.

[6] M. G. Briscoe. Preliminary results from trimoored internal wave experiment (IWEX). Journal of Geophysical Research-Oceans and Atmospheres, 80(27):3872-3884, 1975.

[7] M. G. Brown, J. A. Colosi, S. Tomsovic, A. L. Virovlyansky, M. A. Wolfson, and G. M. Zaslavsky. Ray dynamics in long-range deep ocean sound propagation. Journal of the Acoustical Society of America, 113(5):2533-2547, 2003. 
[8] J. L. Cairns and G. O. Williams. Internal wave observations from a midwater float .2. Journal of Geophysical Research-Oceans and Atmospheres, 81(12):1943-1950, 1976.

[9] C. T. Chen, L. S. Chen, and F. J. Millero. Speed of sound in $\mathrm{NaCl}, \mathrm{MgCl} 2, \mathrm{Na} 2 \mathrm{SO} 4$, and $\mathrm{MgSO} 4$ aqueous-solutions as functions of concentration, temperature, and pressure. Journal of the Acoustical Society of America, 63(6):1795-1800, 1978.

[10] C. T. Chen and F. J. Millero. Speed of sound in seawater at high-pressures. Journal of the Acoustical Society of America, 62(5):1129-1135, 1977.

[11] M. D. Collins. A self-starter for the parabolic equation method. Journal of the Acoustical Society of America, 92(4):2069-2074, 1992.

[12] M. D. Collins. A split-step Pade solution for the parabolic equation method. Journal of the Acoustical Society of America, 93(4):1736-1742, 1993.

[13] M. D. Collins. Generalization of the split-step Pade solution. Journal of the Acoustical Society of America, 96(1):382-385, 1994.

[14] M. D. Collins and E. K. Westwood. A higher-order energy-conserving parabolic equation for range-dependent ocean depth, sound speed, and density. Journal of the Acoustical Society of America, 89(3):1068-1075, 1991.

[15] J. A. Colosi. Geometric sound propagation through an inhomogeneous and moving ocean: Scattering by small scale internal wave currents (l). Journal of the Acoustical Society of America, 119(2):705-708, 2006.

[16] J. A. Colosi and A. B. Baggeroer. On the kinematics of broadband multipath scintillation and the approach to saturation. Journal of the Acoustical Society of America, 116(6):3515-3522, 2004.

[17] J. A. Colosi, A. B. Baggeroer, B. D. Cornuelle, M. A. Dzieciuch, W. H. Munk, P. F. Worcester, B. D. Dushaw, B. M. Howe, J. A. Mercer, R. C. Spindel, T. G. Birdsall, K. Metzger, and A. M. G. Forbes. Analysis of multipath acoustic, field variability and coherence in the finale of broadband basin-scale transmissions in the North Pacific Ocean. Journal of the Acoustical Society of America, 117(3):1538-1564, 2005. Part 2. 
[18] J. A. Colosi and M. G. Brown. Efficient numerical stimulation of stochastic internalwave-induced sound-speed perturbation fields. Journal of the Acoustical Society of America, 103(4):2232-2235, 1998.

[19] J. A. Colosi and S. M. Flatté. Mode coupling by internal waves for multimegameter acoustic propagation in the ocean. Journal of the Acoustical Society of America, 100(6):3607-3620, 1996.

[20] J. A. Colosi and ATOC Grp. A review of recent results on ocean acoustic wave propagation in random media: Basin scales. IEEE Journal of Oceanic Engineering, 24(2):138$155,1999$.

[21] J. A. Colosi and W. Munk. Tales of the venerable Honolulu tide gauge. Journal of Physical Oceanography, 36(6):967-996, 2006.

[22] J. A. Colosi, E. K. Scheer, S. M. Flatté, B. D. Cornuelle, M. A. Dzieciuch, W. H. Munk, P. F. Worcester, B. M. Howe, J. A. Mercer, R. C. Spindel, K. Metzger, T. G. Birdsall, and A. B. Baggeroer. Comparisons of measured and predicted acoustic fluctuations for a 3250-km propagation experiment in the eastern North Pacific Ocean. Journal of the Acoustical Society of America, 105(6):3202-3218, 1999.

[23] J. A. Colosi, F. Tappert, and M. Dzieciuch. Further analysis of intensity fluctuations from a 3252-km acoustic propagation experiment in the eastern North Pacific Ocean. Journal of the Acoustical Society of America, 110(1):163-169, 2001.

[24] R. Dashen, S. M. Flatté, and S. A. Reynolds. Path-integral treatment of acoustic mutual coherence functions for rays in a sound channel. Journal of the Acoustical Society of America, 77(5):1716-1722, 1985.

[25] T. F. Duda, S. M. Flatté, J. A. Colosi, B. D. Cornuelle, J. A. Hildebrand, W. S. Hodgkiss, P. F. Worcester, B. M. Howe, J. A. Mercer, and R. C. Spindel. Measured wave-front fluctuations in 1000-km pulse-propagation in the pacific-ocean. Journal of the Acoustical Society of America, 92(2):939-955, 1992. Part 1.

[26] T. F. Duda, S. M. Flatté, and D. B. Creamer. Modeling meter-scale acoustic intensity fluctuations from oceanic fine-structure and microstructure. Journal of Geophysical Research-Oceans, 93(C5):5130-5142, 1988. 
[27] B. D. Dushaw and J. A. Colosi. Ray tracing for ocean acoustic tomography. APL-UW TM 3-98, Applied Physics Laboratory, University of Washington, Seattle, Washington, 1998. 31pp.

[28] B. D. Dushaw, B. M. Howe, J. A. Mercer, and R. C. Spindel. Multimegameter-range acoustic data obtained by bottom-mounted hydrophone arrays for measurement of ocean temperature. IEEE Journal of Oceanic Engineering, 24(2):202-214, 1999.

[29] M. Dzieciuch, W. Munk, and D. L. Rudnick. Propagation of sound through a spicy ocean, the SOFAR overture. Journal of the Acoustical Society of America, 116(3):1447$1462,2004$.

[30] M. Dzieciuch, P. Worcester, and W. Munk. Turning point filters: Analysis of sound propagation on a gyre-scale. Journal of the Acoustical Society of America, 110(1):135149, 2001.

[31] T. E. Ewart. Acoustic fluctuations in open ocean - measurement using a fixed refracted path. Journal of the Acoustical Society of America, 60(1):46-59, 1976.

[32] T. E. Ewart. Numerical-simulation of the effects of oceanic fine-structure on acoustic transmission. Journal of the Acoustical Society of America, 67(2):496-503, 1980.

[33] T. E. Ewart, C. Macaskill, and B. J. Uscinski. The Mate acoustic frequency cross correlations of intensity. Journal of the Acoustical Society of America, 77(5):1732$1741,1985$.

[34] T. E. Ewart and S. A. Reynolds. Preliminary-results of underwater acoustic measurements at a Mid-ocean site, 1977. Journal of the Acoustical Society of America, 65:S17-S17, 1979.

[35] T. E. Ewart and S. A. Reynolds. The Mid-ocean Acoustic Transmission Experiment, MATE. Journal of the Acoustical Society of America, 75(3):785-802, 1984.

[36] T. E. Ewart, S. A. Reynolds, and D. Rouseff. Determining an ocean internal wave model using acoustic log-amplitude and phase: A Rytov inverse. Journal of the Acoustical Society of America, 104(1):146-155, 1998. 
[37] M. Ewing and J. L. Worzel. Long-range sound transmission. Geol. Soc. Am. Memoir,, 27:1-35, 1948.

[38] C.M. Ferla, M.B. Porter, and Jensen F.B. C-SNAP: Coupled SACLANTCEN normal mode propagation loss model. SACLANTCEN document SM-274, 1993.

[39] R. Ferrari and D. L. Rudnick. Thermohaline variability in the upper ocean. Journal of Geophysical Research-Oceans, 105(C7):16857-16883, 2000.

[40] S. M. Flatté. Wave-propagation through random-media - contributions from ocean acoustics. Proceedings of the Ieee, 71(11):1267-1294, 1983.

[41] S. M. Flatté, R. Leung, and S. Y. Lee. Frequency-spectra of acoustic fluctuations caused by oceanic internal waves and other fine-structure. Journal of the Acoustical Society of America, 68(6):1773-1779, 1980.

[42] S. M. Flatté, S. A. Reynolds, R. Dashen, B. Buehler, and P. Maciejewski. AFAR measurements of intensity and intensity moments. Journal of the Acoustical Society of America, 82(3):973-980, 1987.

[43] S. M. Flatté and R. B. Stoughton. Theory of acoustic measurement of internal wave strength as a function of depth, horizontal position, and time. Journal of Geophysical Research-Oceans, 91(C6):7709-7720, 1986.

[44] S. M. Flatté and R. B. Stoughton. Predictions of internal-wave effects on ocean acoustic coherence, travel-time variance, and intensity moments for very long-range propagation. Journal of the Acoustical Society of America, 84(4):1414-1424, 1988.

[45] C. Garrett and W. Munk. Oceanic mixing by breaking internal waves. Deep-Sea Research, 19(12):823-832, 1972.

[46] C. Garrett and W. Munk. Space-time scales of internal waves - progress report. Journal of Geophysical Research, 80(3):291-297, 1975.

[47] C. Garrett and W. Munk. Internal waves in the ocean. Annual Review of Fluid Mechanics, 11:339-369, 1979. 
[48] D. C. Ghiglia and L. A. Romero. Robust 2-dimensional weighted and unweighted phase unwrapping that uses fast transforms and iterative methods. Journal of the Optical Society of America A-Optics Image Science and Vision, 11(1):107-117, 1994.

[49] M. C. Gregg. A comparison of fine structure spectra from the main thermocline. Journal of Physical Oceanography, 7(33):33-40, 1977.

[50] Akira Ishimaru. Wave Propagation and Scattering in Random Media. Academic Press, New York, 1978.

[51] M. D. Levine. Temperature-fluctuations due to internal waves in presence of finestructure. Transactions-American Geophysical Union, 59(4):289-289, 1978.

[52] M. D. Levine. Internal waves in the ocean - a review. Reviews of Geophysics, 21(5):1206-1216, 1983.

[53] M. D. Levine. The upper ocean internal wave field - influence of the surface and mixed layer. Journal of Geophysical Research-Oceans, 92(C5):5035-5044, 1987.

[54] M. D. Levine. A modification of the Garrett-Munk internal wave spectrum. Journal of Physical Oceanography, 32(11):3166-3181, 2002.

[55] M. D. Levine and J. D. Irish. A statistical description of temperature fine-structure in the presence of internal waves. Journal of Physical Oceanography, 11(5):676-691, 1981.

[56] M. D. Levine, J. D. Irish, T. E. Ewart, and S. A. Reynolds. Simultaneous spatial and temporal measurements of the internal wave field during Mate. Journal of Geophysical Research-Oceans, 91(C8):9709-9719, 1986.

[57] M. D. Levine and V. Zervakis. Near-inertial wave-propagation into the pycnocline during ocean storms - observations and model comparison. Journal of Physical Oceanography, 25(11):2890-2908, 1995.

[58] Flatté Stanley M. Sound Transmission Through a Fluctuating Ocean. Cambridge Monographs on Mechanics and Applied Mathematics. Cambridge University Press, Cambridge [Eng.] ; New York, 1979. 
[59] J. M. Martin and S. M. Flatté. Intensity images and statistics from numericalsimulation of wave-propagation in 3-d random-media. Applied Optics, 27(11):2111$2126,1988$.

[60] J. M. Martin and S. M. Flatté. Simulation of point-source scintillation through 3dimensional random-media. Journal of the Optical Society of America A-Optics Image Science and Vision, 7(5):838-847, 1990.

[61] James Mercer, Rex Andrew, Bruce Howe, and Colosi John. Cruise Report: Long-range Ocean Acoustic PropagationEXperiment(LOAPEX). Technical Report, APL-UW TR 0501, Applied Physics Laboratory, University of Washington, Seattle, Washington, 2005.

[62] A. K. Morozov and J. A. Colosi. Entropy and scintillation analysis of acoustical beam propagation through ocean internal waves. Journal of the Acoustical Society of America, 117(3):1611-1623, 2005. Part 2.

[63] W. Munk and A. Baggeroer. The Heard Island papers - A contribution to global acoustics. Journal of the Acoustical Society of America, 96(4):2327-2329, 1994.

[64] W. Munk and C. Wunsch. Ocean acoustic tomography - scheme for large-scale monitoring. Deep-Sea Research Part A-Oceanographic Research Papers, 26(2):123-161, 1979.

[65] W. Munk and C. Wunsch. Up down resolution in ocean acoustic tomography. Deep-Sea Research Part A-Oceanographic Research Papers, 29(12):1415-1436, 1982.

[66] W. Munk and C. Wunsch. Ocean acoustic tomography - rays and modes. Reviews of Geophysics, 21(4):777-793, 1983.

[67] W. Munk, B. Zetler, J. Clark, S. Gill, D. Porter, J. Spiesberger, and R. Spindel. Tidal effects on long-range sound-transmission. Journal of Geophysical Research-Oceans and Atmospheres, 86(NC7):6399-6410, 1981.

[68] W. H. Munk. Sound channel in an exponentially stratified ocean, with application to SOFAR. Journal of the Acoustical Society of America, 55(2):220-226, 1974.

[69] W. H. Munk. Internal wave spectra at the buoyant and inertial frequencies. Journal of Physical Oceanography, 10(11):1718-1728, 1980. 
[70] W. H. Munk, R. C. Spindel, A. Baggeroer, and T. G. Birdsall. The Heard Island Feasibility Test. Journal of the Acoustical Society of America, 96(4):2330-2342, 1994.

[71] W. H. Munk and F. Zachariasen. Sound-propagation through a fluctuating stratified ocean - theory and observation. Journal of the Acoustical Society of America, 59(4):818-838, 1976.

[72] Walter H. Munk, Peter Worcester, and Carl Wunsch. Ocean Acoustic Tomography. Cambridge monographs on mechanics. Cambridge University Press, Cambridge ; New York, 1995. (some col.).

[73] R. Pinkel. Doppler sonar observations of internal waves - wave-field structure. Journal of Physical Oceanography, 13(5):804-815, 1983.

[74] R. Pinkel. Doppler sonar observations of internal waves - the wavenumber-frequency spectrum. Journal of Physical Oceanography, 14(8):1249-1270, 1984.

[75] S. A. Reynolds, S. M. Flatté, R. Dashen, B. Buehler, and P. Maciejewski. Afar measurements of acoustic mutual coherence functions of time and frequency. Journal of the Acoustical Society of America, 77(5):1723-1731, 1985.

[76] R. Robertson, L. Padman, and M. D. Levine. Fine-structure, microstructure, and vertical mixing processes in the upper ocean in the Western Weddell Sea. Journal of Geophysical Research-Oceans, 100(C9):18517-18535, 1995.

[77] Henry M. Stommel, Bruce Alfred Warren, and Carl Wunsch. Evolution of physical oceanography : scientific surveys in honor of Henry Stommel. MIT Press, Cambridge, Mass., 1981. edited by Bruce A. Warren and Carl Wunsch. chart in pocket. Includes index.

[78] R. B. Stoughton and S. M. Flatté. Acoustic measurements of internal wave rms displacement and rms horizontal current off Bermuda in late 1983. Journal of Geophysical Research-Oceans, 91(C6):7721-7732, 1986.

[79] K. E. Wage, A. B. Baggeroer, and J. C. Preisig. Modal analysis of broadband acoustic receptions at 3515-km range in the North Pacific using short-time Fourier techniques. Journal of the Acoustical Society of America, 113(2):801-817, 2003. 
[80] M. A. Wolfson. Personal Communication. 2007.

[81] M. A. Wolfson and J. L. Spiesberger. Full-wave simulation of the forward scattering of sound in a structured ocean: A comparison with observations. Journal of the Acoustical Society of America, 106(3):1293-1306, 1999.

[82] P. Worcester. North Pacific Acoustic Laboratory: SPICE04 Report. Technical Report, Scripps Institution of Oceanography, San Diego, California, 2005.

[83] P. F. Worcester, B. D. Cornuelle, M. A. Dzieciuch, W. H. Munk, B. M. Howe, J. A. Mercer, R. C. Spindel, J. A. Colosi, K. Metzger, T. G. Birdsall, and A. B. Baggeroer. A test of basin-scale acoustic thermometry using a large-aperture vertical array at 3250-km range in the eastern North Pacific Ocean. Journal of the Acoustical Society of America, 105(6):3185-3201, 1999.

[84] P. F. Worcester, B. D. Cornuelle, and R. C. Spindel. A review of ocean acoustic tomography - 1987-1990. Reviews of Geophysics, 29:557-570, 1991.

[85] P. F. Worcester and R. C. Spindel. North Pacific Acoustic Laboratory. Journal of the Acoustical Society of America, 117(3):1499-1510, 2005.

[86] P. F. Worcester, G. O. Williams, and S. M. Flatté. Fluctuations of resolved acoustic multipaths at short-range in the ocean. Journal of the Acoustical Society of America, 70(3):825-840, 1981. 